\title{
The Mass-Activity relationships in M and K dwarfs. I. Stellar parameters of our sample of $\mathrm{M}$ and $\mathrm{K}$ dwarfs ${ }^{1}$
}

\author{
Éric R. Houdebine ${ }^{1,2}$, D.J. Mullan ${ }^{3}$, J.G. Doyle ${ }^{1}$, Geoffroy de La Vieuville², C.J. Butler ${ }^{1}$, F. \\ Paletou $^{2}$
}

\begin{abstract}
Empirical correlations between stellar parameters such as rotation or radius and magnetic activity diagnostics require estimates of the effective temperatures and the stellar radii. The aim of this study is to propose simple methods that can be applied to large samples of stars in order to derive estimates of the stellar parameters.

Good empirical correlations between Red/Infra-Red colours (e.g. (R-I $\left.)_{C}\right)$ and effective temperatures have been well established for a long time (e.g. Veeder 1974, Bessell 1979, Leggett 1992). The more recent $(\mathrm{R}-\mathrm{I})_{C}$ colour- $T_{e f f}$ correlation using the data of Mann et al. (2015: hereafter M15) and Boyajian et al. (2012: hereafter B12) shows that this colour can be applied as a temperature estimate for large samples of stars. We find that the mean scatter in $T_{\text {eff }}$ relative to the $(\mathrm{R}-\mathrm{I})_{C}-T_{\text {eff }}$ relationship of $\mathrm{B} 12$ and $\mathrm{M} 15$ data is only $\pm 3 \sigma=44.6 \mathrm{~K}$ for $\mathrm{K}$ dwarfs and $\pm 3 \sigma=39.4 \mathrm{~K}$ for $\mathrm{M}$ dwarfs. These figures are small and show that the $(\mathrm{R}-\mathrm{I})_{C}$ colour can be used as a first guess effective temperature estimator for $\mathrm{K}$ and $\mathrm{M}$ dwarfs.

We derive effective temperatures for about $1910 \mathrm{~K}$ and $\mathrm{M}$ dwarfs using the calibration of (R$\mathrm{I})_{C}$ colour- $T_{\text {eff }}$ from B12 and M15 data. We also compiled $T_{\text {eff }}$ and metallicity measurements available in the literature using the VizieR database.

We determine $T_{\text {eff }}$ for 441 stars with previously unknown effective temperatures. We also identified 21 new spectroscopic binaries and 1 triple system from our high resolution spectra.
\end{abstract}

Subject headings: Stars: late-type dwarfs - Stars: late-type subdwarfs - Stars: Fundamental parameters

\section{Introduction}

The determination of fundamental stellar parameters $\left(T_{e f f}, R_{*}\right.$, and $\left.[\mathrm{M} / \mathrm{H}]\right)$ are essential to many astrophysical studies such as the determinations of exoplanet properties, the comparison to stellar interior models, the determinations of Rotation-Activity Correlations (RACs) and another type of magnetic activity related correlations presented in this series of papers, e.g., the Mass-Activity empirical Correlations (MACs thereafter). In exoplanet studies, the precise

\footnotetext{
${ }^{1}$ Armagh Observatory, College Hill, BT61 9DG Armagh, Northern Ireland

${ }^{2}$ Université Paul Sabatier, Observatoire Midi-Pyrénées, Cnrs, Cnes, IRAP, F-31400 Toulouse, France

${ }^{3}$ Department of Physics and Astronomy, University of Delaware, Newark, DE 19716, USA

${ }^{1}$ Based on Gaia DR2 and Hipparcos parallax measurements.
}

determinations of the stellar parameters are required to establish the radius of a transiting planet. Also, the mass of an exoplanet detected with a given orbital period scales as the mass of the star to the power $2 / 3$.

The determinations of estimates of stellar radii are also important in order to obtain RACs and MACs with a minimum amount of scatter (e.g. Houdebine et al. 2017, Houdebine et al. 2019, in preparation). We estimate that the uncertainties in the determination of rotation periods are due to the uncertainties in stellar radii determinations by an amount of about $50 \%$. In the present series of papers, estimates of stellar radii are also central in order to obtain meaningful empirical correlations between magnetic activity diagnostics, i.e. the (Ca II resonance lines and $H_{\alpha}$ surface fluxes or $R_{H K}^{\prime}$ ), and various quantities related to the stellar radius: $L_{B o l}, M_{V}, M_{*}$ or $R_{*}$. In this paper, we focus on quantities which are relevant to de- 
termining values of stellar radii and $T_{\text {eff }} \mathrm{s}$, for a large sample of $1910 \mathrm{~K}$ and $\mathrm{M}$ dwarfs from dK3 to dM7. We shall postpone the discussion of activity indices to Paper II, when the MACs will be constructed.

Stellar parameters of $\mathrm{K}$ and $\mathrm{M}$ dwarfs were sometimes estimated by direct comparison to models (e.g. Paletou et al. 2015, Casagrande et al. 2008). However, in the case of $\mathrm{M}$ dwarfs there are still numerous atomic and molecular lines that are not included in the currently existing models, although there is a continuing effort to improve the models (e.g. Allard et al. 2015). Also the current models do not include possible NLTE effects nor the presence of spots and the chromosphere. The stellar radii obtained from the studies of low-mass eclipsing binaries (LMEBs) indicate that stellar models tend to underpredict the radii (e.g., Kraus et al. 2014, Spada et al. 2013). Mullan \& McDonald (2001) showed that these discrepancies may be due to the high activity levels of the LMEBs. On the contrary, M15 found some consistency between their derived values of $L_{B o l}, R_{*}, M_{*}$ and $T_{\text {eff }}$ and those predicted by their model calculations. Specifically, M15 found that their models systematically over-predict $T_{\text {eff }}$ and underpredict $R_{*}$ by $-2.2 \%$ and $4.7 \%$ respectively for their hotter stars, in agreement with previous comparisons of models and LMEBs and single main-sequence field stars. M15 also found that for values of $T_{\text {eff }}$ below $3500 \mathrm{~K}$, where stars on the main sequence are predicted to be fully convective, $F_{B o l}$ was systematically underestimated by the models at the $0.2 \%$ level. For stars hotter than $3500 \mathrm{~K}$, the models overestimate $F_{B o l}$ by about $0.4 \%$. M15 also reported systematic differences between their model-based masses and those from Delfosse et al. (2000). The models predict systematically lower masses above $0.50 M_{\odot}$ and systematically higher masses below this threshold. They propose that their model-derived masses are more precise than those from the Delfosse et al. (2000) relation.

B12 compared the luminosity-temperature, luminosit radius, temperature-radius and mass-radius empirical relationships for their sample of $33 \mathrm{~K}$ and $\mathrm{M}$ dwarfs to the models of Padova, Dartmouth, BCAH and Yonsei-Yale (Girardi et al. 2000, Dotter et al. 2008, Baraffe et al. 1998, Demarque et al. 2004). They found that the Dartmouth and BCAH models reproduce the trends of their data the best. In their temperature-radius plane they found that there is a lot of scatter in the radius of a star for a given tem- perature. B12 also found that models over-predict temperatures by an average of $6 \%$. For radii less than $0.7 R_{\odot}$ they found that models under-predict radii by about $10 \%$, and the difference between their observations and models increases with decreasing radii up to $\sim 50 \%$ for radii of $\sim 0.4 R_{\odot}$.

Houdebine (2008, Paper VII) found a relatively tight correlation between $[\mathrm{M} / \mathrm{H}]$ and $R_{*}$ in a sample of M2 dwarfs. $[\mathrm{M} / \mathrm{H}]$ decreases with decreasing $R_{*}$ in normal dwarfs and subdwarfs, such that all subdwarfs have very low $[\mathrm{M} / \mathrm{H}]$ and $R_{*}$. In Houdebine et al. (2016a) we performed extensive compilations of $[\mathrm{M} / \mathrm{H}]$ from the literature for late-K, M3 and M4 dwarfs and correlated $[\mathrm{M} / \mathrm{H}]$ and $R_{*}$. These empirical radius- $[\mathrm{M} / \mathrm{H}]$ correlations emphasize that stellar radii diminish markedly with decreasing $[\mathrm{M} / \mathrm{H}]$. These empirical correlations can explain (at least in part) the scatter observed in the temperature-radius plane in B12 and M15.

As far as the current series of papers is concerned, we aim in Houdebine et al. (2019, Paper II, in preparation) to constrain the efficiency of the dynamo mechanisms as a function of stellar radius and mass. An initial empirical correlation was found between the Ca II line mean EW and absolute magnitude $M_{V}$ by Houdebine (1996) for a sample of M2 dwarfs. Later, Houdebine\& Stempels (1997, Paper VI) also found correlations between the CaII line mean EW and $M_{V}$ for a larger sample of M2 dwarfs. In an effort to constrain the dynamo mechanisms at spectral type M2, Houdebine (2011, Paper XV) also found correlations between the Ca II line mean EW, $M_{V}$ and $[\mathrm{M} / \mathrm{H}]$ for a much larger sample of M2 dwarfs. $\mathrm{He}$ also found a correlation between the Ca II line surface fluxes corrected for metallicity effects and radius: $F_{C a I I} \propto R_{*}^{3.6}$. A consequence of this is that $L_{C a I I}$ grows roughly as the power of 5.6 of the stellar radius in their M2 dwarf sample. Similar correlations were found with the $H_{\alpha}$ line diagnostic.

In the current series of papers, preliminary results tifrdicate that the Ca II line luminosity, $L_{C a I I}$, for the samples of low activity stars from Houdebine et al. (2017) increases roughly as the power of 8 of the stellar radius, a value significantly larger than that found in Paper XV. For the active stars, $L_{C a I I}$ increases as $R_{*}$ to the power of 6.6 . This means that the Ca II luminosity depends most sensitively on the stellar radius (or stellar mass). The power law index for $R_{*}$ is much larger (in magnitude) than that of the effect of the corresponding index for the dependence of 
$L_{C a I I}$ on the rotation period. For the latter, the absolute value of the power law index is largest for dM4 stars (where the magnitude is 3.77 , see Houdebine et al. 2017) and smallest for dK5 stars (for which the magnitude is 0.70 ). These results suggest that the efficiency of the dynamo mechanism may depend primarily on the stellar radius (or mass) in M dwarfs.

We stress that the contents of the present paper are only a first step in a two-step process which aims to derive MACs for our complete stellar sample. The second step in this process will be discussed in Paper II. The present paper focusses on obtaining estimates of the data for the "Radius" or "Mass" axis of the MACs. The subsequent paper will focus at first on obtaining reliable data for the "Activity" axis of the MACs. Once reliable data are available for both axes, a search will then be undertaken (in Paper II) to determine the correlation between Radius or Massrelated parameters and Activity-related diagnostics for our stellar sample.

In the aim to gather estimates of the activity parameter for a large sample of $\mathrm{M}$ and $\mathrm{K}$ dwarfs, we are currently gathering high resolution/high $\mathrm{S} / \mathrm{N}$ spectra with the SOPHIE (Haute-Provence Observatory) and NARVAL (Pic-du-Midi Observatory) high resolution spectrographs. We aim to gather about 500 such spectra of stars that have never been observed in high resolution spectroscopy so far. We presently have obtained high resolution spectra for about 350 stars.

The present paper focuses on the determination of effective temperature based mainly on a correlation between the (R-I)c color and $T_{\text {eff }}$. To use this correlation we have relied on the measurements of (R-I)c colors reported by $\mathrm{B} 12$ and $\mathrm{M} 15$. We believe that B12 provide probably the most accurate determinations of $T_{\text {eff }}$ for a sample of $33 \mathrm{~K}$ and $\mathrm{M}$ dwarfs: our belief is based on the fact that the method used by B12 is nearly independent of stellar models. As is widely recognised, models are inevitably subject to uncertainties based on incomplete opacity sources in the calculations. Moreover, the models also assume that LTE conditions hold true throughout the atmosphere. In contrast to B12, the approach used in M15 is to rely to some extent on deriving stellar parameters by taking advantage of certain models. However, the authors of M15 do not rely solely on the models: they also use the constraints which are provided by precision interferometry in order to derive the stellar parameters. Therefore, in the present paper, by relying in part on M15, it is important to note that interferometric measurements of stellar radii provide a first step in the process by means of which we derive stellar parameters. In a second step, we compile $T_{\text {eff }}$ and $[\mathrm{M} / \mathrm{H}]$ measurements from the literature for most of our targets. When we compare our $T_{\text {eff }}$ with the values which already appeared in the literature, we find that the agreement between our results and those in the literature is generally good. As a third step in our process, we also use the GAIA DR2 parallaxes in order to ensure more reliable estimates of the stellar radii.

\section{Selection of the samples of stars}

It is now well established that the red/infra-red colours are well correlated with effective temperatures in late-K and M dwarfs (e.g. Veeder 1974, Mould \& Hyland 1976, Bessell 1979, Leggett 1992, Ramirez \& Melendez 2005, Boyajian et al. 2012, Mann et al. 2015, Houdebine et al. 2017). Based on our previous studies, we have found that the most suitable initial selection parameter when we wish to identify a homogeneous sample of $\mathrm{K}$ or $\mathrm{M}$ dwarfs belonging to a specific sub-type is the (R-I) color: this color is sensitive to $T_{\text {eff }}$, but less so to metallicity (e.g. Leggett 1992, Ramirez \& Melendez 2005). Moreover, broadband colours of high precision (typically of the order of $3 \%$, Leggett 1992) are widely available in the literature for many of the cool dwarfs which are of interest to us.

Observations of $(\mathrm{R}-\mathrm{I})_{C}$ (Cousin's photometric system) or $(\mathrm{R}-\mathrm{I})_{K}$ (Kron photometric system) for our samples of $\mathrm{K}$ and $\mathrm{M}$ dwarfs were taken from the following papers: Eggen (1971), Veeder (1974), Eggen (1974), Rodgers \& Eggen (1974), Eggen (1976a, 1976b), Mould \& Hyland (1976), Eggen (1978), Eggen (1979), Eggen (1980), Weis \& Upgren (1982), Upgren \& Lu (1986), Eggen (1987), Booth et al. (1988), Leggett \& Hawkins (1988), Dawson \& Forbes (1989), Laing (1989), Bessel (1990), Weis (1991a 1991b), Dawson \& Forbes (1992), Leggett (1992), Ryan (1992), Ruiz \& Anguita (1993), Weis (1993), Weis (1996), Reid et al. (2004), Koen et al. (2010).

We selected 8 samples of $K$ and $M$ dwarfs according to their $(\mathrm{R}-\mathrm{I})_{C}$ colours: mid-K sample $\left((\mathrm{R}-\mathrm{I})_{C} \in[0.560: 0.680],(\mathrm{R}-\mathrm{I})_{K} \in[0.400: 0.500], 465\right.$ stars $)$, late-K sample $\left((\mathrm{R}-\mathrm{I})_{C} \in[0.684: 0.816], \quad(\mathrm{R}-\right.$ I $)_{K} \in[0.503: 0.613], 419$ stars $), \quad M 2$ sample ((RI) $)_{C} \in[1.050: 1.240],(\mathrm{R}-\mathrm{I})_{K} \in[0.823: 0.972], 520$ stars $)$, 


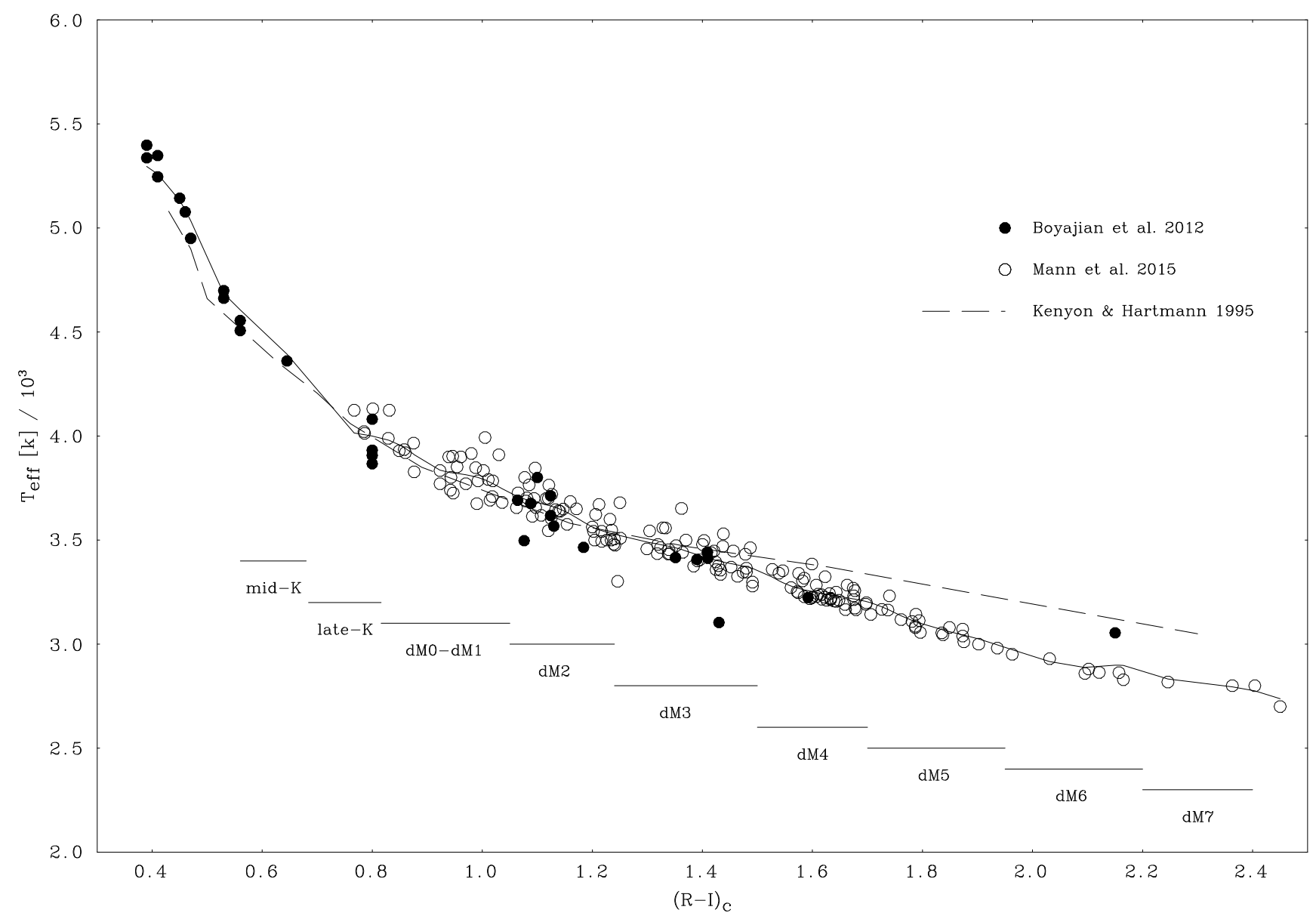

Fig. 1.- Values of $T_{\text {eff }}$ as a function of the (R-I)c color for the data of B12 (filled circles) and M15 (open circles) and a smoothing of these data (dotted line). Also shown for comparison: the calibration of Kenyon and Hartmann (1995). This later calibration agrees well with the more recent measures of Mann et al. (2015) up to $(\mathrm{R}-\mathrm{I}) \mathrm{c}=1.3$, but tends to overestimate $T_{\text {eff }}$ for later spectral types. We overplot the (R-I)c domains of our samples of stars from mid-K to dM7. 
M3 sample $\left((\mathrm{R}-\mathrm{I})_{C} \in[1.240: 1.500],(\mathrm{R}-\mathrm{I})_{K} \in[0.972: 1.180\right.$ troscopy before, from late-K to M5.

767 stars $)$, M4 sample $\left((\mathrm{R}-\mathrm{I})_{C} \in[1.500: 1.700]\right.$, (R-

$\mathrm{I})_{K} \in[1.180: 1.349], 395$ stars $), \quad$ M5 sample ((R-

$\mathrm{I})_{C} \in[1.70: 1.95],(\mathrm{R}-\mathrm{I})_{K} \in[1.349: 1.579], 155$ stars $)$,

M6 sample $\left((\mathrm{R}-\mathrm{I})_{C} \in[1.95: 2.20],(\mathrm{R}-\mathrm{I})_{K} \in[1.579: 1.860]\right.$,

30 stars), M7 sample $\left((\mathrm{R}-\mathrm{I})_{C} \in[2.20: 2.40]\right.$, (RI) $K \in[1.860: 2.216], 14$ stars $)$. This represents a total sample of $2765 \mathrm{~K}$ and $\mathrm{M}$ dwarfs. Most stars in this sample are nearby or large proper motion stars. We report to Leggett (1992) for a detailed desciption of the Cousin's and Kron photometric system as well as for the transformation formulae between the two systems. The error of the transformation from the Kron system and the Cousin's system should be less than 1\% (Leggett 1992). We also completed these samples of stars with a $9^{t h}$ sample of stars: the M0-M1 sample, which includes some stars from the samples of stars from B12 and M15 (see Table 1), as well as several stars from initially the M2 sample which were found to have higher temperatures (we included in the M0-M1 sample, stars down to the spectral type dM1.5).

We note, however, that spectral classification may differ from one author to another. In this regard, in previous Papers on dM1 stars (Houdebine 2008, Paper VII, Houdebine 2009, Paper XII, Houdebine et al. 2009 Paper XIII, Houdebine 2010b Paper IX, Houdebine 2010a Paper XIV, Houdebine 2010c Paper X, Paper XV, Houdebine et al. 2012 Paper XIX), we used a different calibration. According to their infrared colours and the classification of Leggett (1992) these stars are in fact dM2 stars. We shall use in the future this calibration and therefore all our previous work on "dM1" stars in this series of papers should be considered as papers referring to dM2 stars.

Our principal goal in this series of papers is to constrain the Mass-Activity Empirical Correlations by determining the radii of our sample stars as well as the level of magnetic activity in the Ca II and $\mathrm{H}_{\alpha}$ spectral lines. Searching through databases at the European Southern Observatory (ESO) and Observatoire de Haute Provence (OHP), we identified spectra of about 800 different stars which are suitable for our purposes. These spectra will be studied in Houdebine et al. (2019b, in preparation). The spectra of these datadases are completed by our own observations with the spectrgraphs SOPHIE (OHP) and NARVAL (Pic-Du-Midi). These observations include about 430 high resolution spectra of dwarfs never observed in the Ca II lines in high resolution spec-
Here, we focus on the determination of the effective temperature and radius for the stars in our samples. In the samples presented here, we do not include all the stars in the lists above except for the M6 and M7 samples for which all our stars are included in order to obtain better statistics. For our samples, we notably included all stars with determined metallicities. The final list of our samples of stars is provided in Table 2.

\section{Stellar parameters}

Here, we discuss the methods we use to derive $T_{\text {eff }}$ and radius for each of our samples of stars. Reliable estimates of the radii and masses are important if we wish (as in paper II) to determine reliable MassActivity Empirical Correlations.

\subsection{Effective temperatures}

B12 used interferometric observations to determine with high precision (better than $5 \%$ ) the diameters of $33 \mathrm{~K}$ and M dwarfs. They derived empirical correlations linking the effective temperature to broadband colours including (V-R) and (V-I) (from which we derive (R-I)). Their correlations were obtained for stars ranging in spectral type from K0 to M4. Similarly, M15 derived accurate stellar parameters, notably the radii of $\mathrm{K} 7-\mathrm{M} 7$ single stars with a precision of $2-5 \%$, as well as model-independent relations between $T_{\text {eff }}$ and broad-band colours. We show the empirical relationships between $T_{\text {eff } f}$ and the (R-I $)_{C}$ colour in Fig. 1 for these two calibrations. We give the measurements of $(\mathrm{R}-\mathrm{I})_{C}$ and $T_{\text {eff }}$ from B12 and M15 in Table 1. We can see in Fig. 1 that the two parameters are tightly correlated for dwarf stars ranging in spectral type from K0 to M7. We also show the calibration of $T_{e f f}$ as a function of $(\mathrm{R}-\mathrm{I})_{C}$ from Kenyon \& Hartmann (1995) in Fig. 1 for comparison. We overplot a smoothing of the data of B12 and M15 (continuous line). In the present paper, we use the smoothed data (i.e. the continuous line in Fig. 1) to derive "our" values of $T_{\text {eff }}$ for each of the objects in B12 and M15. Once we have derived "our" value of $T_{\text {eff }}$ for each object in both of those papers, we then calculate the temperature differences between our values and those of B12 and M15. We find that the mean of the differences between "our" values and those of B12 and M15 is only $\pm 3 \sigma=44.6 \mathrm{~K}$ for $\mathrm{K}$ dwarfs (23 stars) and $\pm 3 \sigma=39.4 \mathrm{~K}$ for $\mathrm{M}$ dwarfs (161 stars). These values demonstrate that the $(\mathrm{R}-\mathrm{I})_{C}$ color is a reasonable 
temperature diagnostic for most $\mathrm{K}$ and $\mathrm{M}$ dwarfs.

In this regard, we note that the calibration of Kenyon and Hartmann (1995) agrees well with the measures of B12 and M15 up to $(\mathrm{R}-\mathrm{I})_{C}=1.3$, but tends to overestimate $T_{\text {eff }}$ for later spectral types. For comparison between the different spectral subtypes used in this study, we overplot the $(\mathrm{R}-\mathrm{I})_{C}$ domains of our samples of stars in Fig. 1 from mid-K to dM7.

Using the smoothed fit (continuous line in Fig. 1) to the calibrations of B12 and M15, we have determined values of $T_{\text {eff }}$ from our compilations of $(\mathrm{R}-\mathrm{I})_{C}$ measurements for all our target stars (see Tables 1 and 2). In Table 2, values of $T_{\text {eff }}$ which we have derived from our smoothed continuous line in Fig. 1 are listed in column 4.

We also aim in this study to provide an extensive compilation of $T_{\text {eff }}$ compiled from the literature for our objects. The results of this extensive search are listed in column 5 of Table 2 for all stars in our sample.

In order to have a complete compilation of previous measurements of $T_{e f f}$, we queried all catalogs in the VizieR database (CDS, Strasbourg, France: 2). To this respect, we used the tutorial developed by Paletou \& Zolotukhin (20143). The results of the queries provide us with thousands of measurements for several thousands of stars in all our target lists. However, the queries are carried out for all objects around the coordinates of the target stars. For instance, in case of binaries, most often, measurements of the two binary components are included in the compilations. Also, some other brighter or fainter stars (for instance white dwarfs) may be included. As a consequence, many spurious $T_{\text {eff }}$ measurements are included in our compilations. In order to be sure we have only the correct measurements of our target stars, we had to query separately many catalogs individually for many of our targets. Our compilation of temperatures includes all measurements regardless of the methods used to derive them.

The sources of the published effective temperatures for our K dwarf samples are: Blackwell \& LynasGray (1998), Soubiran et al. (1998), Cenarro et al. (2001), Borde et al. (2002), Gray et al. (2003), Le Borgne et al. (2003), Wright et al. (2003), Yong \& Lambert (2003), Clem et al. (2004), Kovtyukh et

\footnotetext{
${ }^{2}$ http://vizier.u-strasbg.fr/viz-bin/VizieR

${ }^{3}$ http://arxiv.org/abs/1408.7026
}

al. (2004), Valenti \& Fischer (2005), Ammons et al. (2006), Casagrande et al. (2006), Gray et al. (2006), Masana et al. (2006), Sanchez-Blazquez et al. (2006), Sousa et al. (2006), Cenarro et al. (2007), Carney et al. (2008), Casagrande et al. (2008), Morales et al. (2008), Soubiran et al. (2008), Sousa et al. (2008), Cortés et al. (2009), da Silva et al. (2009), Jenkins et al. (2009), Mantega et al. (2009), Oenehag et al. (2009), Lafrasse et al. (2010), Soubiran et al. (2010), Valentini \& Munari (2010), Casagrande et al. (2011), Malyuto \& Shvelidze (2011), Prugniel et al. (2011), Wright et al. (2011), McDonald et al. (2012), Bermejo et al. (2013), Molenda-Zakowicz et al. (2013), Pace (2013), Stelzer et al. (2013), Tsantaki et al. (2013), Chen et al. (2014), Cottaar et al. (2014), Franchini et al. (2014), Gaidos et al. (2014), Munari et al. (2014), Kopytova et al. (2016).

For the $\mathrm{M}$ dwarfs, the effective temperatures come from the following authors: Silva \& Cornell (1992), Blackwell \& Lynas-Gray (1998), Cenarro et al. (2001), Malkan et al. (2002), Gray et al. (2003), Le Borgne et al. (2003), Wright et al. (2003), Allende Prieto et al. (2004), Clem et al. (2004), Valdes et al. (2004), Valenti \& Fischer (2005), Ammons et al. (2006), Butler et al. (2006), Gray et al. (2006), Sanchez-Blazquez et al. (2006), Sousa et al. (2006), Cenarro et al. (2007), Schiavon (2007), Baines et al. (2008), Casagrande et al. (2008), Morales et al. (2008), Soubiran et al. (2008), Sousa et al. (2008), Jenkins et al. (2009), Schroeder et al. (2009), van Belle \& von Braun (2009), Brown (2010), Gazzano et al. (2010), Houdebine (2010), Lafrasse et al. (2010), Soubiran et al. (2010), Casagrande et al. (2011), Malyuto \& Shvelidze (2011), Prugniel et al. (2011), Wright et al. (2011), Christiansen et al. (2012), Houdebine (2012), Houdebine et al. (2012), Koleva \& Vazdekis (2012), McDonald et al. (2012), RojasAyala et al. (2012), Bermejo et al. (2013), Cesetti et al. (2013), Lepine et al. (2013), Molenda-Zakowicz et al. (2013), Pace (2013), Rajpurohit et al. (2013), Stelzer et al. (2013), Gaidos et al. (2014), Loyd \& France (2014), Munari et al. (2014), Rajpurohit et al. (2014), Eker et al. (2015), Frasca et al. (2015), Mann et al. (2015), Newton et al. (2015).

We found from these authors that differences in $T_{\text {eff }}$ between different sources are commonly of the order of 100-200 K and may even exceed 400-500 K ! We found that these differences are the greatest among the mid-K, late-K, dM6 and dM7 samples. They are somewhat smaller among the dM0-dM1, 
dM2, dM3, dM4 and dM5 samples. We found for instance that the $T_{\text {eff }}$ measures of Jenkins et al. (2009) and Lepine et al. (2013) are systematically underestimated. We found in general that the $T_{\text {eff }}$ are underestimated for M0-M5 dwarfs compared to our values derived from the $(\mathrm{R}-\mathrm{I})_{C}-T_{\text {eff }}$ calibration of B12 and M15. Nevertheless, our values are in good agreement with those from Gaidos et al. (2014), and may even be slightly underestimated.

We computed the means of the temperature differences between our measures from $(\mathrm{R}-\mathrm{I})_{C}$ and from the literature for all our samples. We find that in average, the $T_{\text {eff }}$ differences are $34.78 \mathrm{~K}, 41.40 \mathrm{~K}$, $22.11 \mathrm{~K}, 29.81 \mathrm{~K}, 25.50 \mathrm{~K}, 27.60 \mathrm{~K}, 32.08 \mathrm{~K}, 17.90 \mathrm{~K}$ and $54.36 \mathrm{~K}$ for our mid-K, late-K, dM0-dM1, dM2, dM3, dM4, dM5, dM6 and dM7 stellar samples respectively. We assigned these values as estimates of the $\pm 3 \sigma$ uncertainty on our measures of $T_{\text {eff }}$ derived from $(\mathrm{R}-\mathrm{I})_{C}$. We can see from these values that the $T_{\text {eff }}$ measurements from the literature are best determined for mid-K, dM3 and dM6 stars, but are worse determined for late-K, dM5 and dM7 stars. We emphasize the relatively large uncertainty on the determinations of $T_{\text {eff }}$ for the dM7 stars (over $\pm 100 \mathrm{~K}$ ). We also would like to emphasize that the differences between our measures of $T_{e f f}$ derived from $(\mathrm{R}-\mathrm{I})_{C}$ and those of the literature directly depends on the number of literature measurements: i.e. the largest are the numbers of measures, the smallests are the differences with our measures. On the contrary when only one measure is available from the literature, the difference with our $T_{\text {eff }}$ derived from $(\mathrm{R}-\mathrm{I})_{C}$ is generally large. The means of the temperature differences provide us with estimates of the $\pm 3 \sigma$ uncertainty on our $T_{\text {eff }}$ determinations from $(\mathrm{R}-\mathrm{I})_{C}$. We stress here that these uncertainties are relatively low (as low as $\pm 18 \mathrm{~K}$ ) compared to the typical $\pm 100 \mathrm{~K}$ uncertainty claimed by most authors. This underlines that the $(\mathrm{R}-\mathrm{I})_{C}$ colour is a reasonable effective temperature diagnostic for most $\mathrm{K}$ and $\mathrm{M}$ dwarfs, and that the calibrations we use in this study are reasonably reliable. We also stress that the $(\mathrm{R}-\mathrm{I})_{C}$ colour give good effective temperature estimates even for sub-dwarfs (e.g. Gl 130, Gl 333, Gl 438, Gl 563.2A, Gl 563.2B, Gl 637 and Gl 817 in the dM2 sample). This highlights the relatively low sensitivity of the $(\mathrm{R}-\mathrm{I})_{C}$ colour on the metallicity. We emphasize that the $(\mathrm{R}-\mathrm{I})_{C}$ colour varies in time for many $\mathrm{M}$ dwarfs due to the presence of spots. This somewhat alters the precision of the $(\mathrm{R}-\mathrm{I})_{C}$ measurements from the literature for active dwarfs. This is especially true for M6 and M7 objects that are most often very active (see Houdebine et al. 2019b, in preparation).

We give in Table 2 the mean temperatures (column 6 ) of the temperatures derived from $(\mathrm{R}-\mathrm{I})_{C}$ (column 4) and the temperatures compiled from the literature (column 5). We also give in Table 2 the uncertainties on our final $T_{\text {eff }}$ measures as the differences between $T_{\text {eff }}$ derived from $(\mathrm{R}-\mathrm{I})_{C}$ and $T_{\text {eff }}$ from the literature. When this value is not available (no measures from the literature), we assigned the uncertainty as the mean of the temperature differences.

\subsection{Effective temperatures: Systematic er- rors}

In order to better estimate the agreement between our final $T_{\text {eff }}$ and the $T_{\text {eff }}$ from previous authors, we plot these two parameters in Fig. 2. In Fig. 2, the continuous (solid) line indicates the correlation which would exist if there was perfect correspondence between the two approaches we have used to evaluate $T_{\text {eff }}$ : (i) from the literature, and (ii) from the color-temperature relationships obtained by B12 and M15. It can be seen that the agreement between the datasets is rather good. The scatter of the measures around the mean curve is typically lower than $100 \mathrm{~K}$ at all spectral subtypes. We note that this scatter is lower for instance for mid-K dwarfs than for late-K dwarfs. This better agreement among mid-K dwarfs is due mostly to the larger number of $T_{\text {eff }}$ measurements from the literature for these stars. On the other hand, in general we have fewer estimates of $T_{\text {eff }}$ from the literature for late-K dwarfs. We again stress that a large part of the scatter observed in Fig. 2 is due to few measures of $T_{\text {eff }}$ from the literature. When large numbers of measures of $T_{\text {eff }}$ are available from the literature (e.g. for the Gliese stars), the agreement with our $T_{\text {eff }}$ derived from the $(\mathrm{R}-\mathrm{I})_{C}$ color is generally good. We also note that the scatter is lower among $\mathrm{M}$ dwarfs compared to late-K dwarfs and especially for M4 to M7 stars.

The data in Figure 2 show that, for late-K dwarfs, when we compare the $T_{\text {eff }}$ values obtained by the two methods, the literature values of $T_{\text {eff }}$ are on average larger by about $6 \%$ than the values of $T_{\text {eff }}$ which are obtained from the B12 calibration. In contrast to such behavior, the results in Figure 2 indicate that for mid-K dwarfs, we find that the literature values of $T_{\text {eff }}$ are on average smaller by about $9 \%$ than the B12 values. Also for $\mathrm{M}$ dwarfs, the behavior is sim- 


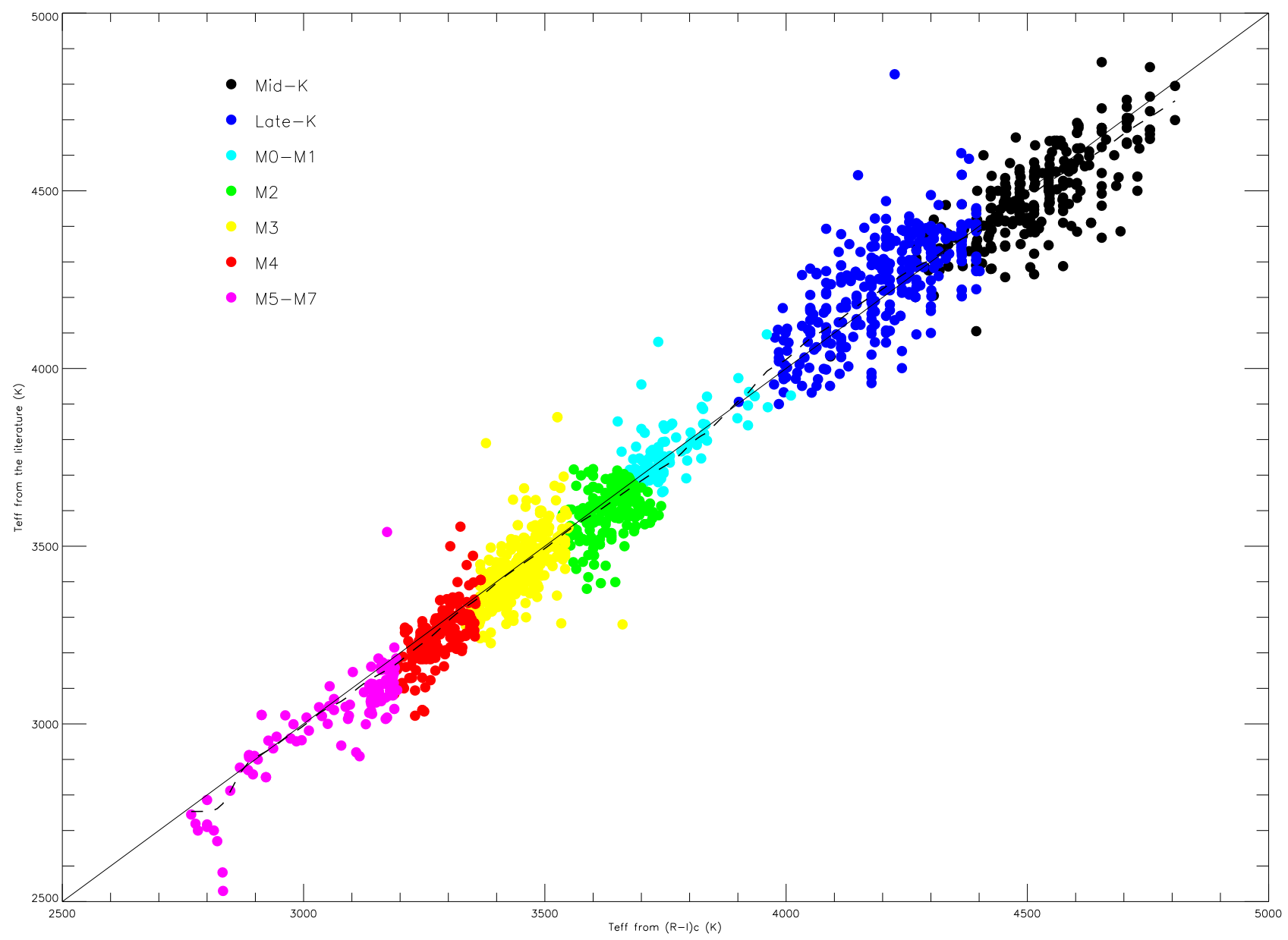

Fig. 2.- Comparison between two sets of values of $T_{\text {eff }}$ : (i) values taken from the literature; and (ii) values which have been derived from the $(\mathrm{R}-\mathrm{I})_{C} / T_{\text {eff }}$ correlations which have been derived by M15 and B12. Continuous line: the correlation which would exist if there was an exact one-to-one correspondence between the two sets of $T_{\text {eff }}$. Dashed line: the best fit correlation when the data are smoothed by a Gaussian of FWHM $30 \mathrm{~K}$. Note that there exists a good overall agreement between the smoothed data and the expectations of a one-to-one correspondence. 
ilar to that for the mid-K stars, i.e. the literature values of $T_{\text {eff }}$ are smaller by typically $5 \%$ than the B12 values. We note that Mann et al. (2013) also reported that their effective temperatures were slightly larger than those from other authors in the literature by about $50 \mathrm{~K}$. M15 found that their estimated $T_{\text {eff }}$ were typically $100 \mathrm{~K}$ warmer than those from the work of Casagrande et al. (2008). On the other hand, Mann et al (2013) reported on a good agreement between their $T_{\text {eff }}$ estimates and those of Rojas-Ayala et al. (2012). During the course of our data compilation, it is worth noting that we found good agreement between our $T_{\text {eff }}$ values derived from $(\mathrm{R}-\mathrm{I})_{C}$ and the recent estimates of $T_{\text {eff }}$ reported by Gaidos et al. (2014).

As an example of a compilation of temperatures from the literature, we list in Table 3 the temperatures obtained by 6 different teams of authors for various sub-sets of the stars which overlap with those in our dM4 sample. For the 6 teams as listed from left to right in Table 3 , the number $\mathrm{O}(\mathrm{i})$ of stars which overlap with our sample are $\mathrm{O}(\mathrm{i})=38,30,11,32$, 60, and 111 respectively: the largest overlap (111) is with the team of Gaidos et al (2014), while the smallest overlap (11) is with the team of Wright et al (2011). We list, at the bottom of Table 3, the mean temperatures obtained by those 6 teams for their respective sub-set of dM4 stars. We also list, for each team $(i=16)$ the mean temperatures which we have obtained for the dM4 stars in our sample (numbering $\mathrm{O}(\mathrm{i})$ ) which overlap with the stars observed by Team (i). We also list the mean temperature differences between our values and those of the 6 authors: the differences are listed in absolute terms (in degrees $\mathrm{K}$ ) and also as a percentage. These figures highlight the systematic differences between the results obtained by means of our approach and those obtained by 6 other sets of authors.

We note that, in the case of two of the 6 teams, there is very good agreement, $\pm 0.3 \%$ on average, between our estimates of $T_{\text {eff }}$ and theirs: these are the teams of Gaidos et al. (2014) and of Stelzer et al. (2013). To understand why there is such a good overlap between ourselves and Gaidos et al. (2014), we note that Gaidos et al. followed the procedure of Mann et al. (2013) in order to estimate $T_{\text {eff }}, R_{*}$ and luminosity $L_{*}$ for their sample. Gaidos et al. first determined $T_{\text {eff }}$ by finding the best-fitting model stellar spectrum, and then they used the best-fitting temperature in empirical relations to determine the other stellar parameters. This procedure was calibrated against nearby stars with known radii, distances and bolometric fluxes (Boyajian et al. 2012). Therefore, their method is essentially the same as that used by M15: since the approach we adopt in the present paper also relies in part on M15, it is perhaps not too surprising that we have found a very good agreement with the results obtained by Gaidos et al (2014). Moreover, the overlap of stars between our sample and the sample of Gaidos et al is the largest (111 stars), and this may also contribute to improving the agreement between our results.

On the other hand, the good agreement that we have found with the results of Stelzer et al. (2013) cannot be attributed to similarity of approach. In fact, Stelzer et al. (2013) used a completely different technique based on the spectral type calibration reported by Lépine et al. (2012). The latter calibration is based on the (V-J) color, and is therefore quite distinct from the method (based on the $(R-I)_{C}$ color) which we have used in the present paper. Once Stelzer et al. had determined the spectral type for each star, they then used the temperature scale reported by Bessell (1991) and Mohanti \& Basri (2003) in order to derive $T_{e f f}$. In view of the difficulties associated with assigning a precise spectral type and then also converting to a temperature, this method is expected to be subject to several uncertainties. Nevertheless, as shown in Table 3, it is encouraging to see that we have actually found good overall agreement between the Stelzer et al. temperatures and ours. And we note that the overlap between our sample of dM4 stars and those of Stelzer et al. is only 32: thus, we cannot claim that the largeness of the example might be helping to bring our samples into agreement.

Turning now to the results of a third team, we note that Morales et al. (2008) have used the TiO5 index to derive spectral types for their sample of $\mathrm{M}$ dwarfs: the TiO5 index is based on the strongest $\mathrm{TiO}$ feature in $\mathrm{M}$ dwarf spectra, with a bandhead at $7050 \AA$. Effective temperatures were computed using the spectral-type temperature correlation reported by Bessel (1991). Morales et al. used an iterative procedure to ensure that their $T_{\text {eff }}$ values were consistent with the bolometric correction in the $\mathrm{K}$ band $B C_{K}$. We find (see Table 3 ) that the values of $T_{\text {eff }}$ obtained by Morales et al. for M4 dwarfs lie about $84 \mathrm{~K}$ below our own measures for the dM4 sample. We believe that this significant difference in $T_{\text {eff }}$ values can 
be attributed to the presence of a systematic difference between the calibration of Bessel (1991, see also Leggett et al. 1996), and the calibration of M15.

The team of Jenkins et al. (2009) used the $\left(\mathrm{V}-\mathrm{K}_{S}\right)$ $T_{\text {eff }}$ relation reported by Casagrande et al. (2008). The latter investigators claimed a typical internal uncertainty of $\pm 17 \mathrm{~K}$. In preparation for using the Casagrande et al relation, Jenkins et al. first computed the absolute $\mathrm{V}$ and $\mathrm{K}$ magnitudes. The $\mathrm{V}$ magnitudes were taken from SIMBAD, while the $K_{S}$ magnitudes were taken from the 2MASS catalog (Skrutskie et al. 2006). We find that, on average, the $T_{\text {eff }}$ values obtained by Jenkins et al. are lower than ours by about $212 \mathrm{~K}$. This figure is larger than the differences we have found relative to those of other teams. For this reason, we have rejected some of the Jenkins et al results in cases where the difference was significantly larger than the mean $T_{\text {eff }}$ values obtained by ourselves and by other teams.

The team of Wright et al. (2011) have used a combination of techniques in order to estimate the temperatures for their targets. For most stars, they adopted two methods, both involving isochrones from Siess et al. (2000): (i) for cluster stars, they combined the isochrones with an estimate of the age of the appropriate cluster; (ii) for field stars, they assumed an age of $1 \mathrm{Gyr}$. For the field stars with unknown distances, the $(\mathrm{V}-\mathrm{K})$ color led them to derive a temperature using the relationships of Casagrande et al. (2008) for M dwarfs, and those of Casagrande et al. (2010) for FGK stars. Their results for M4 dwarfs are found to lie about $171 \mathrm{~K}$ hotter than our measures $(+5.19 \%)$. The study by the Wright et al. (2011) team is the only one of the six teams which clearly overestimates the temperatures in midM dwarfs compared to the tabulation of Mann et al. (2015). We note that the sample of Wright et al (2011) has the smallest overlap with our sample of dM4 stars: only 11 stars contribute to the overlap. This smallness of overlap may serve to enhance the difference in the mean values of $T_{e f f}$.

Finally, the team of Lépine et al. (2013) have obtained results for the most complete survey of $M$ dwarfs in the northern sky. They determined the stellar parameters using a complex method based on fitting stellar atmosphere models from Allard et al. (2011). They fitted their spectra in the wavelength range $5600-9000 \AA$, but excluding the problematic $\mathrm{TiO}$ bands between 6400 and $6600 \AA$. Between the average values of $T_{\text {eff }}$ obtained by of Lẽpine et al. and the averages which we have obtained, there is a systematic difference of $-91 \mathrm{~K}(-2.78 \%)$.

By taking a grand average of the 6 mean differences in $T_{\text {eff }}$ in Table 3 , we find a value of $-36 \mathrm{deg}$. $\mathrm{K}$. Compared to the typical $T_{\text {eff }}$ values listed in Table 3 , this grand average amounts to a fractional error of about $1 \%$.

As we saw above, the rather good agreement between our values of $T_{\text {eff }}$ and those in the literature may be considered as somewhat surprising in view of the systematic differences between the various approaches adopted by the different authors. We believe that the sources of these systematic differences may include (but are not limited to) the following: missing opacities, the LTE assumption in the models, and metallicity effects on the $(\mathrm{R}-\mathrm{I})_{C}$ color. Differences of typically $100 \mathrm{~K}$ or $200 \mathrm{~K}$ between different authors are common in our compilation of effective temperatures, mainly because of systematic errors. However, it appears that on average, these differences cancel one another to a greater or lesser extent, thereby yielding values which are not so far removed from our calibration based on the work of B12 and M15. Without doubt, we can assert that the best reference is the work of B12.

It is true that we have found some discrepancies between the literature values of $T_{\text {eff }}$ and our values in the mean mid-K and late-K subsamples. These discrepancies may point to systematic errors in the methods used to derive values of $T_{\text {eff }}$ in the literature. Because our compilation includes works by authors who have relied on many different techniques, we believe it is beyond the scope of this paper to discuss in detail all possible sources of uncertainties in detail. We only can say that in average, systematic errors for individual stars are typically less than $10 \%$ , while the errors which appear in the grand average value of $T_{\text {eff }}$ are of order $1 \%$. In the case of the $\mathrm{K}$ dwarfs, we find that there are systematic errors of about $5 \%$ for the $T_{\text {eff }}$ values which we derive for $\mathrm{K}$ dwarfs: we find that the sign of the systematic error is positive for late-K stars and negative for mid-K stars.

For $\mathrm{M}$ dwarfs, our results for $T_{\text {eff }}$ are based mostly on the approach which was developed by M15. The uncertainties associated with their results have already been discussed in the Introduction. The M15 work seems to be one of the most precise studies so far, essentially because it includes results from interferometry. Since we rely heavily on M15, we recog- 
nize that whatever sources of error contribute to the results in M15 also apply to our results. Moreover, we find that our $T_{\text {eff }}$ values are systematically larger than the literature values for most authors, with the notable exception of Gaidos et al. (2014). We have found that the systematic differences in $T_{e f f}$ are of order 5\% for M4 and M5 dwarfs. However, the percentage errors are larger for the less well known M7 dwarfs. On the other hand, we have found good agreement for the M2 and M3 subsamples.

In summary, our results for $T_{\text {eff }}$ include systematic errors of about $5 \%$ overestimates, in addition to which we should allow for the uncertainties in the results of M15 (see Introduction).

In Table 2, the errors given in column 6 are the differences between the estimated effective temperature and the solid line curve in Fig. 1. This definition means that the difference is between our $T_{\text {eff }}$ value and the mean of the values of $T_{\text {eff }}$ in the literature. However, it is important to note that errors on the literature values of $T_{\text {eff }}$ may be as large as $200 \mathrm{~K}$ ! Therefore the mean errors on our samples given above are only statistical measures of the mean of the scatter relative to the straight line in Fig. 2. It is difficult at this stage to estimate absolute errors for our $T_{\text {eff }}$ values since many different sources contribute to the errors.

Another additional source of systematic error is the effect of $[\mathrm{M} / \mathrm{H}]$ on the $(\mathrm{R}-\mathrm{I})_{C}$ color. Leggett (1992) found that for a given $(\mathrm{R}-\mathrm{I})_{C}$ color, there exists one spectral subtype difference between Young Disk and Halo $\mathrm{M}$ dwarfs. In other words, for a given $(\mathrm{R}-\mathrm{I})_{C}$, low metallicity $\mathrm{M}$ dwarfs tend to be cooler than solar metallicity dwarfs. The consequence is that our values of $T_{\text {eff }}$ derived from (R-I) $C$ tend to be cooler for subdwarfs. This is one reason why we have compiled metallicities for our targets. Even if this systematic trend has been well established by other authors, we find (see Table 2) that in general, for the vast majority of our subdwarfs the difference between our $T_{\text {eff }}$ values and the values in the literature remains small, except for very low metallicity subdwarfs (e.g. the sdM2 VB 12).

Moreover, it is also a fact that the presence of surface inhomogeneities on the surface of an M dwarf (especially on a dMe star) can have an effect on the value of $T_{\text {eff }}$ which is measured for such a star. This effect applies to our data, as well as values of $T_{\text {eff }}$ which appear in the literature. Systematic errors due to this effect can hardly be determined with any re- liability unless full investigations of the spectral variations in time have been established. We note also that there are some variations of the $(\mathrm{R}-\mathrm{I})_{C}$ colors in our compilations, which may yield uncertainties up to about $100 \mathrm{~K}$ for certain objects. For the best known objects, we have sometimes found several reported measurements of $(\mathrm{R}-\mathrm{I})_{C}$, and for such objects, we use the mean. Such variability in the actual color of a particular star can be responsible for a significant part of the scatter in Fig. 2,

We show in Fig. 3 the values of our final effective temperatures as a function of $(\mathrm{R}-\mathrm{I})_{C}$ for our complete samples of stars. One can see in this figure that the correlation between $T_{e f f}$ and $(\mathrm{R}-\mathrm{I})_{C}$ is good. This confirms that $(\mathrm{R}-\mathrm{I})_{C}$ is a good first guess effective temperature diagnostic for $\mathrm{K}$ and $\mathrm{M}$ dwarfs. We show the heteroscedastic least-square fit to the data as the solid line. Weights are proportional to the inverse of the error on the final temperature determination. This least square fit of order 5 yields:

$$
\begin{aligned}
T_{\text {eff }} / 3500= & -2.3365 \times(R-I)_{C}^{5} \\
& +4.1056 \times(R-I)_{C}^{4} \\
& -3.7582 \times(R-I)_{C}^{3} \\
& +2.4905 \times(R-I)_{C}^{2} \\
-0.06876 \times & (R-I)_{C}+0.646327
\end{aligned}
$$

The $\chi^{2}$ is $3.56 \times 10^{-6}$ for this fit, which is good. Most stars in our sample lie within $\pm 100 \mathrm{~K}$ of the least squares fit. This fit could be used as a complementary calibration of $T_{\text {eff }}$ as a function of $(\mathrm{R}-\mathrm{I})_{C}$ for $\mathrm{K}$ and M dwarfs as it is based on both the work of B12 and M15, and our complete compilation of $T_{\text {eff }}$ from the literature.

\subsection{Stellar radii}

In order to derive radii for our samples of stars, we used the classical formula e.g. Lang 1980);

$$
M_{v}+B C_{V}=42.36-5 \times \log \left(\frac{R_{*}}{R_{\odot}}\right)-10 \times \log \left(T_{e f f}\right),
$$

where symbols take their usual meaning. We used the $B C_{V}$ calibration as a function of $T_{e f f}$ from Lejeune et al. (1998). We assumed that their tabulation yields an uncertainty of about $10 \%$ in $B C_{V}$. Using Eq. (2), we obtained the radii for our samples of stars as listed in Table 2. The errors on the radii in Table 2 were 


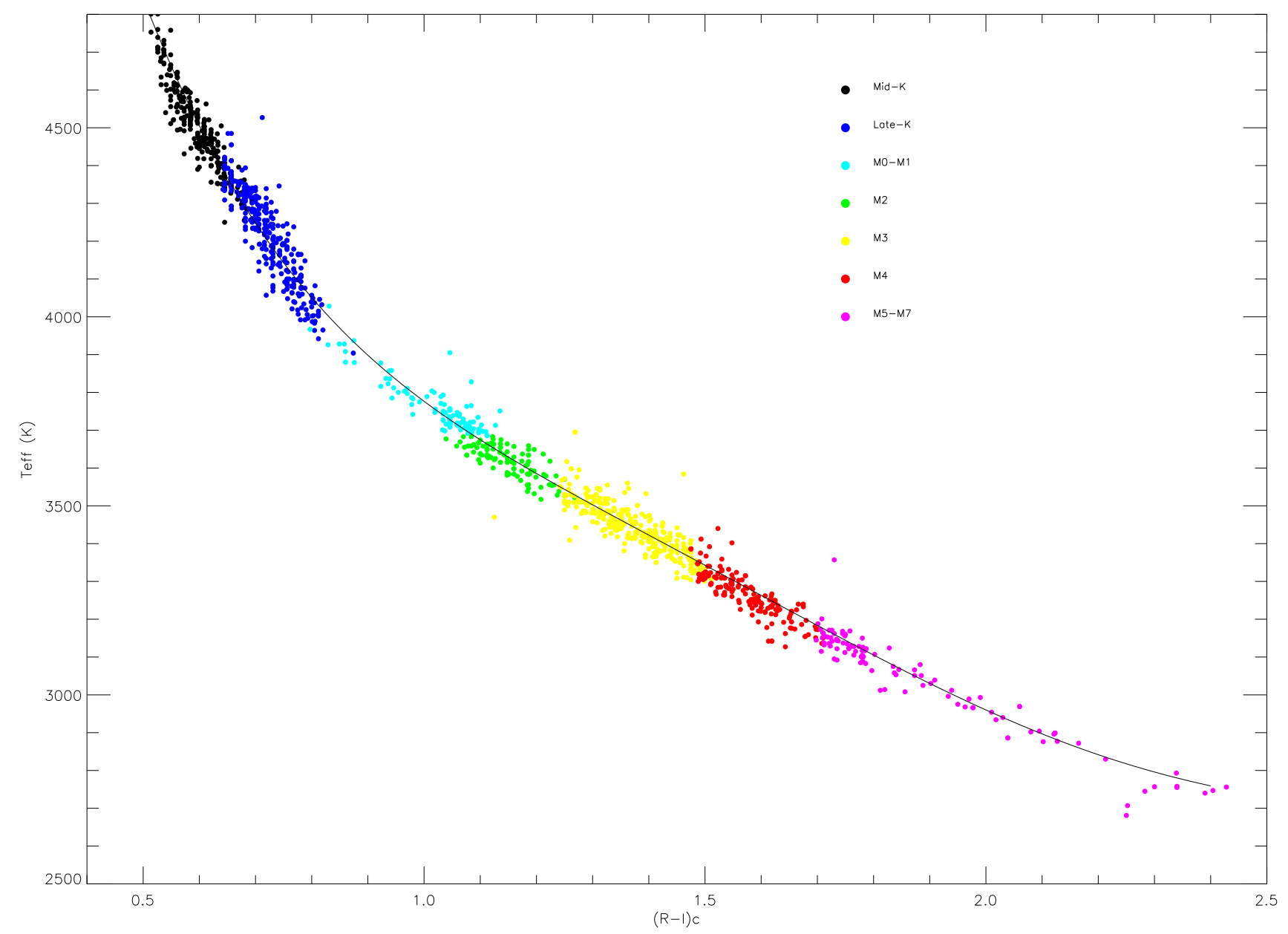

Fig. 3. - Values of $T_{\text {eff }}$ compiled in the present study as a function of $(\mathrm{R}-\mathrm{I})_{C}$ for our complete samples of stars. Solid line: The heteroscedastic least-square fit to the data (see text). 
computed taking into account the combination of the error on the absolute magnitude, an assumed error of 0.02 magnitudes on the $(\mathrm{R}-\mathrm{I})_{C}$ colour, an error of 0.005 magnitudes when $V$ is known to 3 decimals, 0.02 magnitudes when $V$ is known to 2 decimals, and 0.2 magnitudes when $V$ is known to 1 decimal. We also took into account the errors on $T_{\text {eff }}$.

Our source for the parallaxes comes mostly from the recent GAIA DR2 compilation (GAIA collaboration 2016, 2018). Other sources are Jenkins (1952), Gliese \& Jahreiss (1991), Van Altena et al. (1995), Salim \& Gould (2003), Costa et al. (2005), van Leeuwen (2007), Subasavage et al. (2009), Dieterich et al. (2014), Lurie et al. (2014). The errors on the parallaxes were included in our calculations of the $V$ band absolute magnitude $M_{V}$. We found that the GAIA DR2 data allows a tremendous improvement on the precision of the stellar radii especially over the older data of Gliese \& Jahreiss (1991). In some instances we found radii different by a factor of 2 or 3 between these two sources. We also found some significant differences with the Hipparcos parrallaxes in many instances, notably for faint Hipparcos sources.

We show the stellar radii as a function of $T_{\text {eff }}$ in Fig. 4 for all of the (1910) stars in our samples. We show the weighted least squares fit as the continuous line for stars restricted to $-0.5 \leq[M / H] \leq+0.5$. The dashed line represents the polynomial fit determined by B12 for their sample of (33) K and M stars. We also show as the dot-dashed line, the polynomial fit obtained by M15, for their sample of (183) K and $\mathrm{M}$ dwarfs. For our present samples of stars we obtained the following weighted polynomial fit:

$$
\begin{array}{r}
R_{*}=2.8102 \times X^{6}-7.5796 \times X^{5}+1.2666 \times X^{4} \\
+9.0658 \times X^{3}-2.8046 \times X^{2}-3.9200 \times X \\
+1.5606
\end{array}
$$

where $X=T_{\text {eff }} / 3500$.. The weights on each data point were assigned as the inverse of the errors on the radii.

We overplot for each of our spectral sub-type domain the mean of the errors on $T_{\text {eff }}$ as well as the mean of the errors on $R_{*}$. We show these error bars in the lower part of Fig. 4.

The first striking characteristic of the data represented in Fig. 4 is the very large scatter among the radii for a given effective temperature. This scatter can be attributed partly to uncertainties on $T_{\text {eff }}$ and
$R_{*}$ as can be seen on the means of the error bars plotted in Fig. 4. However, the scatter is still significantly larger than these typical error bars, notably for the earliest $\mathrm{K}$ dwarfs in our sample as well as the early $\mathrm{M}$ dwarfs. Such a large scatter in the radii was also observed among M dwarfs by Mann et al. (2015). They showed that the differences between radii at a given effective temperature is mainly due to differences in metallicities: stars with large radii are metal rich and stars with small radii are metal poor (e.g. subdwarfs). Houdebine (2008) found that there exists an empirical correlation between stellar radius and metallicity among his sample of M2 dwarfs. Later, Houdebine et al. (2016) found also analogous empirical correlations among their samples of late-K, M3 and M4 dwarfs. Although it appeared in this later study that for M4 dwarfs, the dependency of the stellar radius on the metallicity is somewhat smaller than in say, late-K or M2 dwarfs, it seems that both parameters do correlate among $\mathrm{K}$ and $\mathrm{M}$ dwarfs. The smallest dependency of late $\mathrm{M}$ dwarf radii on metallicity may explain why we observe a smaller scatter in Fig. 4 for spectral types equal or greater than M5, compared to earlier spectral type $M$ dwarfs. Note also, that there are probably some (so far) unidentified binary stars in our samples (stars with abnormally large radii), and that our samples also contain subdwarfs.

We find a generally good agreement between our fit and the fits of B12 and M15 from late-K to M3. However, we note some differences for early-K stars and stars later than M3. For M4 and particularly M5, the B12 curve lies by $40 \%$ or more below our fit and the fit of M15. The fit from M15 agrees well with our fit from M1 to M5 stars, which is not surprising since for $\mathrm{M}$ dwarfs our temperature determinations depends for $50 \%$ on their calibration, and the other $50 \%$ depend on our literature compilation. For lower effective temperatures, the fit of M15 lies above our fit by up to about $70 \%$. Undoubtedly our sample is larger than that of M15 by a factor of 12 and larger than the B12 sample by a factor of 80 . If we trust that the B12 and M15 temperature determinations give "accurate" values, and that our final temperature determinations rely on a compilation from all other authors by up to $50 \%$, we may be tempted, considering our much larger sample, that our fit should yield a better description of the temprature-radius plane. But this is somewhat open to discussion since the errors on the temperatures may still be large.

We note that our fit do not reproduce well the radii 


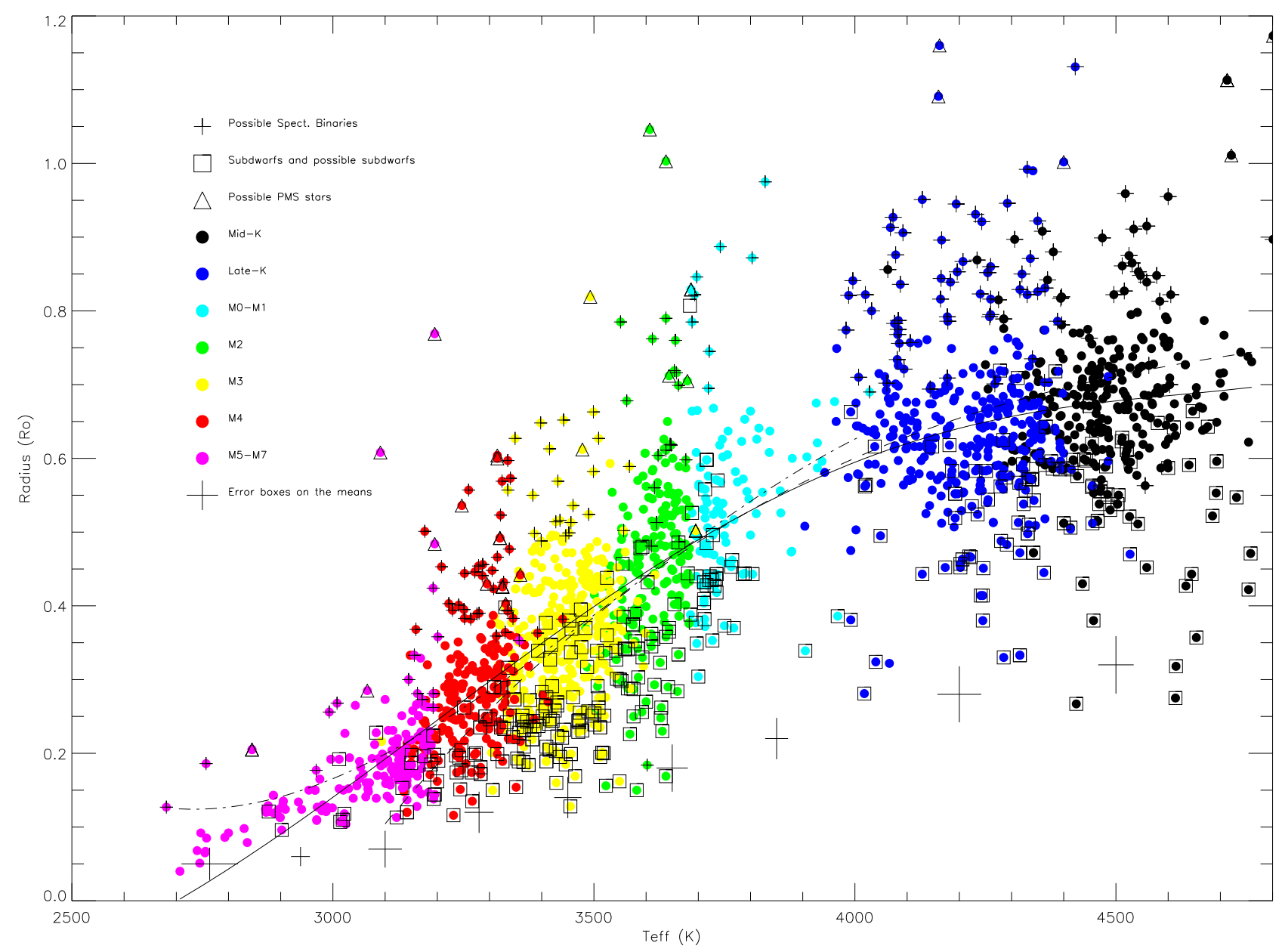

Fig. 4. - Stellar radius as a function of $T_{\text {eff }}$ for our samples of $\mathrm{K}$ and $\mathrm{M}$ dwarfs. We show the heteroscedastic least square fit as the continuous line. The dashed line shows the fit from Boyajian et al. (2012). The dotdashed line represents the fit determined by Mann et al. (2015) for their sample of stars. The large scatter which is present in this figure is due (in large part) to the effects of different values of metallicity among the individual stars in any sample. 
of M6 and M7 dwarfs. This is due to the fact that errors on the radii for those stars are proportionally larger than those in M4 and M5 dwarfs, and as a result the weights assigned to M6 and M7 dwarfs are lower. The much larger samples of M4 and M5 dwarfs "force" the slope of the fit at M6 and M7 to be larger than expected.

The GAIA DR2 parallaxes allow a major improvement on the precision of the stellar radii of our nearby stars. Before, uncertainties on the parallaxes accounted for a large part of the uncertainties on the radii, especially for the faint $M$ dwarfs. In the present study, we find that the largest uncertainty now arise from the uncertainty of the effective temperatures. The better precision on the radii now allows us to isolate possible spectroscopic binaries or stars with large metallicities which stand significantly above the main trend, and probable low metallicity stars (probable subdwarfs) that stand significantly below the main trend in the radius-temperature plane. The comparison of the radii of those stars with those of stars in our samples with $[M / H]<-0.5$ or $[M / H]>+0.5$ (from our compilation of $[\mathrm{M} / \mathrm{H}]$ from the literature), indicates the stars with possible metallicities below -0.5 or above +0.5 . We show the known and possible metal poor stars as squares in Fig. 4. We also show in this figure, the possible metal rich and/or spectroscopic binaries which display abnormally large radii. We indentified 211 possible low metallicity dwarfs, 184 metal rich and/or spectroscopic binaries. We also found 30 possible PMS dwarfs which have abnormally large radii and are known to be neither metal rich nor spectroscopic binaries according to our high resolution spectra. From the analysis of our high resolution spectra, we have identified 21 new spectroscopic binaries and 1 new triple system.

Our correlation between radius and $T_{\text {eff }}$ can be compared to the Hertzsprung-Russell diagrams for nearby stars from the GAIA Collaboration (2018b). They note a steepening of the slope at about $M_{G} \sim 11$ in their Fig. 6. Our data do not show a clear evidence for a similar increase in the slope in Fig. 4. The Radius- $T_{\text {eff }}$ diagrams in Rabus et al. (2019) show that there are two possible regimes in their diagrams and they propose that their results are possible evidence for a change between partially convective stars and fully convective stars (The Transition To Complete Convection, TTCC). They locate this transition at about $T_{\text {eff }} \sim 3300 \mathrm{~K}$. This result agrees with the finding of Houdebine et al. (2017) who found that the RACs undergo important changes between dM2 and dM3 where they located the TTCC. This finding suggests that important changes occur in the dynamo mechanisms at the TTCC.

\section{Conclusion}

From our compilations of $(\mathrm{R}-\mathrm{I})_{C}$ measures from the literature, we report on new derivations of effective temperatures for $1910 \mathrm{~K}$ and $\mathrm{M}$ dwarfs, based on the calibrations of B12 and M15. We have also compiled previously published effective temperatures for most stars in our sample. We derived our final effective temperature estimates by computing the mean of the effective temperatures derived from the (R$\mathrm{I})_{C}$ colour and those from the literature. We find in general a good agreement between our temperatures derived from $(\mathrm{R}-\mathrm{I})_{C}$ and the published effective temperatures (see Table 2).

We derive a new heteroscedastic polynomial fit to the $T_{\text {eff }}$ versus (R-I) $C$ correlation. Our correlation is based both on the B12 and M15 calibrations and our compilation of $T_{\text {eff } f}$ from the litterature for thousands of measurements. We find a rather good $(\mathrm{R}-\mathrm{I})_{C}-T_{\text {eff }}$ correlation in agreement with the results from previous authors.

The recent release of the GAIA DR2 data allows us to compute the stellar radii for most of the targets in our stellar sample. We now find that the largest uncertainties on the radii arise from the uncertainties on the effective temperatures. We are currently carrying out a long term observing program dedicated to the measurements of chromospheric lines in $\mathrm{M}$ and $\mathrm{K}$ dwarfs. So far, we have identified 21 new spectroscopic binaries and 1 triple system. We find that the precision on the stellar radii may allow to identify new low metallicity stars as well as new high metallicity and/or spectroscopic binaries. Our data suggest that 425 such new objects may be present in our data sets.

\section{Acknowledgements}

This research has made use of the SIMBAD database, operated at CDS, Strasbourg, France. DJM is supported in part by the NASA Space Grant program. This research made use of Astropy, a community-developed core Python package for Astronomy (Astropy Collaboration, 2013). This research has made use of the VizieR catalogue access

${ }^{4}$ http://www.astropy.org/ and http://astroquery.readthedocs.org/en/latest/ 
tool, CDS, Strasbourg, France. The original description of the VizieR service was published in A\&AS $143,23$.

This work has made use of data from the European Space Agency (ESA) mission Gaia

(https://www.cosmos.esa.int/gaia), processed by the Gaia Data Processing and Analysis Consortium (DPAC, https://www.cosmos.esa.int/web/gaia/dpac/cons Funding for the DPAC has been provided by national institutions, in particular the institutions participating in the Gaia Multilateral Agreement.

\section{REFERENCES}

Allard, F., Homeier, D., Freytag, B. et al. 2013, Memorie della Societa Astronomica Italiana Supplementi, 24, 128

Allende Prieto C., Barklem P.S., Lambert D.L., Cunha K. 2004, A\&A 420, 183

Ammons, S.M., Robinson, S.E., Strader, J., Laughlin, G., Fisher, D., \& Wolf, A, 2006, ApJ. 638, 1004

Baines E.K., Mcalister H.A., Ten Brummelaar T.A., Turner N.H., Sturmann J., Sturmann L., Goldfinger P.J., Ridgway S.T. 2008, Astrophys. J., 680, 728

Baraffe, I., Chabrier, G., Allard, F. \& Hauschildt, P.H. 1998, A\&A 337, 403

Bermejo, J. M.; Asensio Ramos, A.; Allende Prieto, C. 2013, A\&A 553, 95

Bessel, M.S. 1979, PASP 91, 589.

Bessel, M.S. 1990, A\&A Sup. Ser. 83, 357

Bessel, M.S. 1991, AJ 101, 662

Blackwell D.E. \& Lynas-Gray A.E. 1998, A\&AS 129, 505

Booth, J., Caruso, J., Weis, E.W. 1988, PASP 100, 749

Borde P., Coude du Foresto V., Chagnon G., Perrin G. 2002, A\&A 393, 183

Boyajian, T.S.; von Braun, K.; van Belle, G.; McAlister, H.A.; ten Brummelaar, T.A.; Kane, S.R.; Muirhead, P.S.; Jones, J.; White, R.; Schaefer,
G.; Ciardi, D.; Henry, T.; Lpez-Morales, M.; Ridgway, S.; Gies, D.; Jao, Wei-Chun; Rojas-Ayala, B.; Parks, J.R.; Sturmann, L.; Sturmann, J.; Turner, N.H.; Farrington, C.; Goldfinger, P.J.; Berger, D.H. 2012, ApJ 757, 112 (B12)

Brown T.M. 2010, Astrophys. J., 709, 535

Butler R.P., Wright J.T., Marcy G.W., Fischer D.A., rtizamt. S.S., Tinney C.G., Jones H.R.A., Carter B.D., Johnson J.A., McCarthy C., Penny A.J. 2006, Astrophys. J., 646, 505

Carney B.W.; Latham D.W.; Stefanik R.P.; Laird J.B. 2008, AJ 135, 196

Casagrande L., Portinari L., Flynn C. 2006, MNRAS, $373,13-44$

Casagrande L., Flynn C., Bessell M. 2008, MNRAS, 389,585

Casagrande, L., Ramirez, I., Meléndez, J., Bessell, M. \& Asplund, M., 2010, A\&A 512, 54

Casagrande L., Schoenrich R., Asplund M., Cassisi S., Ramirez I., Melendez J., Bensby T., Feltzing S. 2011, Astron. Astrophys. 530, A138

Cenarro, A.J., Cardiel, N., Gorgas, J., Peletier, R.F., Vazdekis, A., Prada, F. 2001, MNRAS 326, 959

Cenarro A.J., Peletier R.F., Sanchez-Blazquez P., Selam S.O., Toloba E., Cardiel N., Falcon-Barroso J., Gorgas J., Jimenez-Vicente J., Vazdekis A. 2007, Mon. Not. R. Astron. Soc., 374, 664

Cesetti M., Pizzella A., Ivanov V.D., Morelli L., Corsini E.M., Dalla Bonta E. 2013, Astron. Astrophys., 549, A129

Chen C.H., Mittal T., Kuchner M., Forrest W.J., Lisse C.M., Manoj P., Sargent B.A., Watson D.M. 2014, ApJ. Suppl. Ser., 211, 25

Christiansen J.L., Jenkins J.M., Caldwell D.A., Burke C.J., Tenenbaum P., Seader S., Thompson S.E., Barclay T.S., Clarke B.D., Li J., Smith J.C., Stumpe M.C., Twicken J.D., Van Cleve J. 2012, Publ. Astron. Soc. Pac., 124, 1279

Clem J.L.; Vandenberg D.A.; Grundahl F.; Bell R.A. 2004, AJ 127, 1227 
Cortés, C.; Silva, J.R.P.; Recio-Blanco, A.; Catelan, M.; Do Nascimento, J.D., Jr.; De Medeiros, J.R. 2009, ApJ 704, 750

Cottaar M., Covey K.R., Meyer M.R., Nidever D.L., Stassun K.G., Foster J.B., Tan J.C., Chojnowski S.D., da Rio N., Flaherty K.M., Frinchaboy P.M., Skrutskie M., Majewski S.R., Wilson J.C., Zasowski G. 2014 ApJ 794, 125

Costa, Edgardo; Mndez, Ren A.; Jao, W.-C.; Henry, Todd J.; Subasavage, John P.; Brown, Misty A.; Ianna, Philip A.; Bartlett, Jennifer 2005, AJ 130, 337

da Silva L., Torres C.A.O., de la Reza R., Quast G.R., Melo C.H.F., Sterzik M.F. 2009, Astron. Astrophys., 508, 833

Dawson, P.C., Forbes, D. 1989, PASP 101, 614

Dawson, P.C., Forbes, D. 1992, AJ 103, 2063

Delfosse, X., Forveille, T., Ségransan, D. et al. 2000, A\&A, 364, 217

Demarque, P., Woo, J.-H., Kim, Y.-C. \& Yi, S.K. 2004, ApJS 155, 667

Dieterich, Sergio B.; Henry, Todd J.; Jao, Wei-Chun; Winters, Jennifer G.; Hosey, Altonio D.; Riedel, Adric R.; Subasavage, John P. 2014, AJ 147, 94

Dotter, A., Chaboyer, B., Jevremovic, D. et al. 2008, ApJS 178, 89

Eggen, O.J., 1971, ApJS 22, 389

Eggen, O.J., 1974, PASP 86, 697

Eggen, O.J., 1976a, ApJS 30, 351

Eggen, O.J., 1976b, ApJ 204, 101

Eggen, O.J., 1978, ApJS 37, 251

Eggen, O.J., 1979, ApJS 39, 89

Eggen, O.J., 1980, ApJS 43, 457

Eggen, O.J., 1987, AJ 92, 379

Eker Z., Soydugan F., Soydugan E., Bilir S., Yaz Gokce E., Steer I., Tuysuz M., Senyuz T., Demircan O. 2015, Astron. J., 149, 131
Franchini M., Morossi C., Marcantonio P.D., Malagnini M.L., Chavez M. 2014, MNRAS, 442, 220

Frasca A., Biazzo K., Lanzafame A.C., Alcala J.M., Brugaletta E., Klutsch A., Stelzer B., Sacco G.G., Spina L., Jeffries R.D., Montes D., Alfaro E.J., Barentsen G., Bonito R., Gameiro J.F., LopezSantiago J., Pace G., Pasquini L., Prisinzano L., Sousa S.G., Gilmore G., Randich S., Micela G., Bragaglia A., Flaccomio E., Bayo A., Costado M.T., Franciosini E., Hill V., Hourihane A., Jofre P., Lardo C., Maiorca E., Masseron T., Morbidelli L., Worley C.C. 2015, A\&A 575, A4

Gaia Collaboration et al., 2016: Description of the Gaia mission (spacecraft, instruments, survey and measurement principles, and operations); Astronomy \& Astrophysics, Volume 595, id.A1.

Gaia Collaboration, Babusiaux, C., van Leeuwen, F., Barstow, M.A. et al. 2018b, Gaia Data Release 2: Observational Hertzsprung-Russell Diagrams. A\&A 616, A10 (special issue for Gaia DR2).

Gaia Collaboration, A.G.A. Brown, A. Vallenari, T. Prusti, J.H.J. de Bruijne, C. Babusiaux, C.A.L. Bailer-Jones, M. Biermann, D.W. Evans, L. Eyer, F. Jansen, C. Jordi, et al. 2018, Gaia Data Release 2: Summary of the contents and survey properties. A\&A (special issue for Gaia DR2). External Links: Gaia Data Release 2 Documentation release 1.0.

Gaidos E., Mann A.W., Lepine S., Buccino A., James D., Ansdell M., Petrucci R., Mauas P., Hilton E.J. 2014, Mon. Not. R. Astron. Soc., 443, 2561

Gazzano J.-C., de Laverny P., Deleuil M., RecioBlanco A., Bouchy F., Moutou C., Bijaoui A., Ordenovic C., Gandoli D., Loeillet B. 2010, A\&A 523, A91

Girardi, L., Bressan, A., Berteli, G. \& Chiosi, C. 2000, A\&AS, 141, 371

Gliese, W. \& Jahreiss, H. 1991, Catalog of Nearby Stars, Preliminary $3^{r d}$ Version, Astron. RechenInstitut, Heidelberg, VizieR V/70A/catalog.

Gray R.O., Corbally C.J., Garrison R.F., Mcfadden M.T., Robinson P.E. 2003, AJ, 126, 2048

Gray R.O., Corbally C.J., Garrison R.F., McFadden M.T., Bubar E.J., McGahee C.E., O’Donoghue A.A., Knox E.R. 2006, AJ, 132, 161 
Houdebine, E.R. 1996, Stellar surface structure: proceedings of the 176th Symposium of the International Astronomical Union, held in Vienna, Austria, October 9-13, 1995. Edited by Klaus G. Strassmeier and Jeffrey L. Linsky. IAU Symposium 176, Kluwer Academic Publishers, Dordrecht, p. 547

Houdebine, E.R. 2008, MNRAS 390, 1081 (Paper VII)

Houdebine, E.R. 2009, MNRAS 397, 2133 (Paper XII)

Houdebine, E.R. 2010a, MNRAS 407, 1657 (Paper XIV)

Houdebine, E.R. 2010b, A\&A 509, 65 (Paper IX)

Houdebine, E.R. 2010c, MNRAS 403, 2157 (Paper X)

Houdebine, E.R. 2011b, MNRAS 411, 2259 (Paper XV)

Houdebine, E.R. 2012, MNRAS 421, 3189 (Paper XVIII)

Houdebine, E.R., Butler, C. J.; Garcia-Alvarez, D.; Telting, J. 2012, MNRAS 426, 1591 (Paper XIX)

Houdebine, E.R. \& Doyle, G.J. 1994, in Cool Stars, Stellar Systems and the Sun, Eigth Cambridge Workshop, Proceedings, held in Athens, Georgia, Caillault J.P. ed., ASPCS, Vol. 64, p. 423

Houdebine, E.R. \& Stempels, H.C. 1997, A\&A (Paper VI)

Houdebine, E.R., Mullan, D.J., Paletou, F. \& Gebran, M. 2016a, ApJ 822, 97

Houdebine, E.R., Mullan, D.J., Bercu, B., Paletou, F. \& Gebran, M. 2017, ApJ 837, 96

Jenkins, L.F. 1952, The General Catalog of Trigonometric Stellar Parallaxes, New Haven, Yale University Observatory

Jenkins, I.S., Ramsey, L.W., Jones, H.R.A., Pavlenko, Y., Gallardo, I., Barnes, J.R. \& Pinfield, D.I. 2009, ApJ, 704, 975

Kenyon, S.J. \& Hartmann, L. 1995, ApJ Suppl. Ser. 101,117

Koen, C., Kilkenny, D., van Wyk, F. \& Marang, F. 2010, MNRAS 403, 1949
Koleva M., Vazdekis A. 2012, Astron. Astrophys. 538, A143

Kopytova T.G., Brandner W., Tognelli E., Prada Moroni P.G., Da Rio N., Roeser S., Schilbach E. 2016, A\&A 585, A7

Kovtyukh V.V., Soubiran C., Belik S.I. 2004, A\&A, 427,933

Kraus A.L., Shkolnik E.L., Allers K.N., Liu M.C. 2014, Astron. J., 147, 146

Lafrasse S., Mella G., Bonneau D., Duvert G., Delfosse X., Chelli A. 2010, SPIE Conf. on Astronomical Instrumentation $77344 \mathrm{E}$

Laing, J.D. 1989, South African Astron. Obs. Circular, No. 13, 29

Le Borgne, J.-F.; Bruzual, G.; Pell, R.; Lanon, A.; Rocca-Volmerange, B.; Sanahuja, B.; Schaerer, D.; Soubiran, C.; Vlchez-Gmez, R. 2003, A\&A 402, 433

Lang, K.R., 1980, "Astrophysical Formulae", Springer-Verlag, ISBN 3-540-09933-6.

Leggett, S.K., 1992, ApJS 82, 351

Leggett, S.K., Hawkins, M.R.S. 1988, MNRAS 234, 1065

Leggett, S.K., Allard, F., Berriman, G., Dahn, C.C., Hauscildt, P.H., 1996, ApJS 104, 117

Lejeune, T., Cuisinier, F. \& Buser, R. 1998, A\&A 130,65

Lepine S., Hilton E.J., Mann A.W., Wilde M., RojasAyala B., Cruz K.L., Gaidos E. 2013, AJ, 145, 102

Loyd R.O.P., France K. 2014, Astrophys. J. Suppl. Ser., 211, 9

Lurie, John C.; Henry, Todd J.; Jao, Wei-Chun; Quinn, Samuel N.; Winters, Jennifer G.; Ianna, Philip A.; Koerner, David W.; Riedel, Adric R.; Subasavage, John P. 2014, AJ 148, 91

Malkan M.A., Hicks E.K., Teplitz H.I., McLean I.M., Sugai H., Guichard J. 2002, ApJS 142, 79

Malyuto V., Shvelidze T. 2011, Baltic Astron. 20, 89 Mann A.W., Brewer J.M., Gaidos E., Lepine S., Hilton E.J. 2013, Astron. J., 145, 52 
Mann A.W., Feiden G.A., Gaidos E., Boyajian T., Von Braun K. 2015, Astrophys. J., 804, 64 (M15)

Manteiga M., Carricajo I., Rodriguez A., Dafonte C., Arcay B. 2009, AJ 137, 3245

Masana E., Jordi C., Ribas I. 2006, Astron. Astrophys. 450,735

McDonald I., Zijlstra A.A., Boyer M.L. 2012, Mon. Not. R. Astron. Soc., 427, 343

Molenda-Zakowicz J., Sousa S.G., Frasca A., Uytterhoeven K., Briquet M., Van Winckel H., Drobek D., Niemczura E., Lampens P., Lykke J., Bloemen S., Gameiro J.F., Jean C., Volpi D., Gorlova N., Mortier A., Tsantaki M., Raskin G. 2013, MNRAS 434,1422

Morales J.C., Ribas I., Jordi C. 2008, A\&A 478, 507

Mould, J.R. \& Hyland, A.R. 1976, ApJ 208, 399

Mullan, D.J. \& MacDonald, J. 2001, ApJ 559, 353.

Munari, U.; Henden, A.; Frigo, A.; Zwitter, T.; Bienaym, O.; Bland-Hawthorn, J.; Boeche, C.; Freeman, K. C.; Gibson, B. K.; Gilmore, G.; Grebel, E. K.; Helmi, A.; Kordopatis, G.; Levine, S. E.; Navarro, J. F.; Parker, Q. A.; Reid, W.; Seabroke, G. M.; Siebert, A.; Siviero, A.; Smith, T.C.; Steinmetz, M.; Templeton, M.; Terrell, D.; Welch, D.L.; Williams, M.; Wyse, R.F.G. 2014, AJ 148, 81

Newton E.R., Charbonneau D., Irwin J., Mann A.W. 2015, ApJ, 800, 85 L10

Oenehag A.; Gustafsson B.; Eriksson K.; Edvardsson B. 2009, A\&A 498, 527

Pace, G. 2013, A\&A 551, L8

Paletou, F. \& Zolotukhin, I. 2014, arXiv:1408.7026

Paletou, F., Gebran, M.; Houdebine, E. R.; Watson, V. 2015b, A\&A 580A, 78

Prugniel P., Vauglin, I., Koleva M. 2011, Astron. Astrophys. 531, A165

Rabus, M., Lachaume, R., Jordán, A., Brahm, R. Boyajian, T., von Braun, K., Espinoza, N., Berger, U.-P., Le Bouquin, J.-B., Absil, O., 2018, arXiv:1901.08077
Rajpurohit, A.S., Reyle C., Allard F., Homeier D., Schultheis M., Bessell M.S., Robin A.C. 2013, A\&A, 556, A15

Rajpurohit A.S., Reyle C., Allard F., Scholz R.-D., Homeier D., Schultheis M., Bayo A. 2014, Astron. Astrophys. 564, A90

Ramirez, I., Melendez, J. 2005, ApJ 626, 465

Reid, I.N., Cruz, K.L., Allen, P., Mungall, F., Kilkenny, D., Liebert, J., Hawley, S.L., Fraser, O.J., Covey, K.R., Lowrance, P., Kirkpatrick, J.D., \& Burgasser, A.J. 2004, AJ 128, 463

Rodgers, A.W., Eggen, O.J., 1974, PASP 86, 742

Ruiz, M.T. \& Anguita, C. 1993, AJ 105, 614

Rojas-Ayala B., Covey K.R., Muirhead P.S., Lloyd J.P. 2012, Astrophys. J., 748, 93

Ryan, S.G. 1992, AJ 104, 1144

Salim, S. \& Gould, A. 2003, ApJ 582, 1011

Sanchez-Blazquez P., Peletier R.F., Jimenez-Vicente J., Cardiel N., Cenarro A.J., Falcon-Barroso J., Gorgas J., Selam S., Vazdekis A. 2006, Mon. Not. R. Astron. Soc., 371, 703

Schiavon R.P. 2007, Astrophys. J. Suppl. Ser., 171, 146

Silva D.R., Cornell M.E. 1992, Astrophys. J. Suppl. 81,865

Schroeder, C., Reiners, A. \& Schmitt, J.H.M.M. 2009, A\&A 493, 1099

Soubiran C., Katz D.; Cayrel R. 1998, A\&AS 133, 221

Soubiran C., Bienayme O.; Mishenina T.V.; Kovtyukh V.V. 2008, A\&A 480, 91

Soubiran C., Le Campion J.-F., Cayrel de Strobel G., Caillo A. 2010, Astron. Astrophys. 515, A111

Sousa S.G., Santos N.C., Israelian G., Mayor M., Monteiro M.J.P.F.G. 2006, Astron. Astrophys. 458,873

Sousa S.G.; Santos N.C.; Mayor M.; Udry S.; Casagrande L.; Israelian G.; Pepe F.; Queloz D.; Monteiro M.J.P.F.G. 2008, A\&A 487, 373 
Spada, F.; Demarque, P.; Kim, Y.-C.; Sills, A. 2013, ApJ 776, 87

Stelzer B., Marino A., Micela G., Lopez-Santiago J., Liefke C. 2013, Mon. Not. R. Astron. Soc., 431, 2063

Skrutskie, M.F. et al. 2006, AJ, 131, 1163

Subasavage, John P.; Jao, Wei-Chun; Henry, Todd J.; Bergeron, P.; Dufour, P.; Ianna, Philip A.; Costa, Edgardo; Mndez, Ren A. 2009, AJ 137, 4547

Tsantaki M.; Sousa S.G.; Adibekyan V.Zh.; Santos N.C.; Mortier A.; Israelian G. 2013, A\&A 555A, 150

Upgren, A.R., Lu, P.K. 1986, AJ 92, 903

Valdes F., Gupta R., Rose J.A., Singh H.P., Bell D.J. 2004, ApJ Suppl. Ser., 152, 251

Valenti J.A., Fischer D.A. 2005, Astrophys. J. Suppl. Ser., 159, 141

Valentini M., Munari U. 2010, A\&A, 522, A79

van Altena, W.F., Lee, J.T., Hoffleit, E.D. 1995, The General Catalog of Trigonometric Stellar Parallaxes, Fourth Edition, New Haven, Yale University Observatory

van Belle G.T., von Braun K. 2009, ApJ, 694, 1085

van Leeuwen, F. 2007, A\&A 474, 653

Veeder, G.J. 1974, AJ 79, 1056

Weis, E.W. 1991a, AJ 101, 1882

Weis, E.W. 1991b, AJ 102, 1795

Weis, E.W. 1993, AJ 105, 1962

Weis, E.W. 1996, AJ 112, 2300

Weis, E.W, Upgren, A.R. 1982, PASP 94, 821

Wright C.O., Egan M.P., Kraemer K.E., Price S.D. 2003, Astron. J., 125, 359

Wright, N.J., Drake, J.J., Mamajek, E.E., Henry, G.W. 2011, ApJ 743, 48

Yong D., Lambert D.L. 2003, PASP 115, 22

This 2-column preprint was prepared with the AAS LATEX macros v5.2. 
Table 1:: Effective temperatures and $(\mathrm{R}-\mathrm{I})_{C}$ colors for $\mathrm{K}$ and $\mathrm{M}$ dwarfs. In Column 3 , we list the $T_{e f f}$ values we have obtained in the present paper using our $(\mathrm{R}-\mathrm{I})_{C^{-}}$ $T_{\text {eff }}$ calibration. In Columns 4 and 5 , we list the $T_{e f f}$ values reported by M15 and by $\mathrm{B} 12$ respectively.

\begin{tabular}{|c|c|c|c|c|c|}
\hline Star & $\begin{array}{c}(\mathrm{R}-\mathrm{I})_{C} \\
(\mathrm{mag})\end{array}$ & $\begin{array}{c}T_{e f f}{ }^{1} \\
(\mathrm{~K})\end{array}$ & $\begin{array}{c}T_{e f f}{ }^{2} \\
(\mathrm{~K})\end{array}$ & $\begin{array}{c}T_{e f f}^{3} \\
(\mathrm{~K})\end{array}$ & $\begin{array}{c}T_{e f f} \text { Diff } \\
\quad(\mathrm{K})\end{array}$ \\
\hline $\mathrm{Gl} 702 \mathrm{~A}$ & - & - & - & 5407 & - \\
\hline Gl 75 & 0.39 & 5346 & - & 5398 & +52 \\
\hline Gl 631 & 0.39 & 5346 & - & 5337 & -9 \\
\hline Gl 53A & 0.41 & 5259 & - & 5348 & +89 \\
\hline Gl 764 & 0.41 & 5259 & - & 5246 & -13 \\
\hline Gl 166A & 0.45 & 5085 & - & 5202 & +117 \\
\hline Gl 144 & 0.46 & 5041 & - & 5077 & +36 \\
\hline Gl 33 & 0.47 & 4998 & - & 4950 & -48 \\
\hline Gl 892 & 0.53 & 4737 & - & 4699 & -38 \\
\hline Gl 105A & 0.53 & 4737 & - & 4662 & -75 \\
\hline Gl 845 & 0.56 & 4606 & - & 4555 & -51 \\
\hline Gl 570A & 0.563 & 4598 & - & 4507 & -91 \\
\hline Gl $702 \mathrm{~B}$ & 0.620 & 4456 & - & 4393 & -63 \\
\hline $\mathrm{Gl} 820 \mathrm{~A}$ & 0.644 & 4396 & - & 4451 & +55 \\
\hline Gl 673 & 0.767 & 4086 & 4124 & - & +38 \\
\hline Gl $820 B$ & 0.785 & 4040 & 4021 & - & -19 \\
\hline PM $23099+1425$ & 0.786 & 4038 & 4013 & - & -25 \\
\hline Gl 380 & 0.80 & 4002 & - & 4115 & +113 \\
\hline Gl 820B & 0.80 & 4002 & - & 3954 & -48 \\
\hline Gl 338A & 0.80 & 4002 & - & 3907 & -95 \\
\hline Gl 380 & 0.801 & 4000 & 4131 & - & +131 \\
\hline Gl 488 & 0.829 & 3962 & 3989 & - & +27 \\
\hline Gl 169 & 0.831 & 3960 & 4124 & - & +164 \\
\hline gL 172 & 0.849 & 3935 & 3929 & - & -6 \\
\hline Gl 56.3B & 0.858 & 3923 & 3935 & - & +12 \\
\hline Gl 338A & 0.860 & 3921 & 3920 & - & -1 \\
\hline Gl 208 & 0.875 & 3901 & 3966 & - & +65 \\
\hline Gl 525 & 0.876 & 3899 & 3828 & - & -71 \\
\hline $\mathrm{Gl} 338 \mathrm{~B}$ & - & - & - & 3867 & - \\
\hline Gl 281 & 0.923 & 3836 & 3771 & - & -65 \\
\hline Gl 642 & 0.923 & 3836 & 3834 & - & -2 \\
\hline Gl 458.2 & 0.939 & 3828 & 3900 & - & +72 \\
\hline Gl 875 & 0.942 & 3825 & 3740 & - & -85 \\
\hline Gl 239 & 0.943 & 3824 & 3801 & - & -23 \\
\hline PM 22290+0139 & 0.946 & 3822 & 3903 & - & +81 \\
\hline Gl 471 & 0.947 & 3821 & 3726 & - & -95 \\
\hline GJ 3108 & 0.954 & 3815 & 3852 & - & +37 \\
\hline Gl 79 & 0.961 & 3809 & 3900 & - & +91 \\
\hline Gl 838.3B & 0.970 & 3802 & 3771 & - & -31 \\
\hline Gl 864 & 0.980 & 3793 & 3916 & - & +123 \\
\hline Gl 846 & 0.988 & 3787 & 3848 & - & +61 \\
\hline Gl $678.1 \mathrm{~A}$ & 0.990 & 3785 & 3675 & - & -110 \\
\hline Gl 709 & 0.992 & 3783 & 3785 & - & +2 \\
\hline Gl 740 & 1.002 & 3775 & 3834 & - & +59 \\
\hline Gl 459.3 & 1.005 & 3772 & 3993 & - & +221 \\
\hline Gl 809 & 1.011 & 3767 & 3791 & - & +24 \\
\hline Gl 353 & 1.014 & 3764 & 3692 & - & -72 \\
\hline Gl 505B & 1.018 & 3760 & 3709 & - & -51 \\
\hline Gl 96 & 1.019 & 3759 & 3785 & - & +26 \\
\hline Gl 894.1 & 1.030 & 3749 & 3910 & - & +161 \\
\hline Gl 277.1 & 1.036 & 3744 & 3681 & - & -63 \\
\hline GJ 3408B & 1.062 & 3720 & 3656 & - & -64 \\
\hline Gl 809 & 1.064 & 3718 & - & 3692 & -26 \\
\hline Gl 514 & 1.065 & 3717 & 3727 & - & +10 \\
\hline $\mathrm{Gl} 412 \mathrm{~A}$ & 1.076 & 3707 & - & 3497 & -210 \\
\hline Gl 205 & 1.077 & 3706 & 3801 & 3801 & +95 \\
\hline Gl 887 & 1.079 & 3704 & 3688 & - & -16 \\
\hline Gl 272 & 1.081 & 3703 & 3703 & - & +0 \\
\hline Gl 212 & 1.085 & 3699 & 3765 & - & +66 \\
\hline Gl 887 & 1.088 & 3696 & - & 3676 & -20 \\
\hline Gl 701 & 1.091 & 3693 & 3614 & - & -79 \\
\hline Gl 649 & 1.094 & 3691 & 3700 & - & +9 \\
\hline Gl 685 & 1.096 & 3689 & 3846 & - & +157 \\
\hline Gl 686 & 1.097 & 3688 & 3657 & - & -31 \\
\hline $\mathrm{Gl} 412 \mathrm{~A}$ & 1.107 & 3679 & 3619 & - & -60 \\
\hline
\end{tabular}


Table 1:: continued.

\begin{tabular}{|c|c|c|c|c|c|}
\hline Star & $\begin{array}{c}(\mathrm{R}-\mathrm{I})_{c} \\
(\mathrm{mag})\end{array}$ & $\begin{array}{c}T_{\text {eff }}{ }^{1} \\
(\mathrm{~K})\end{array}$ & $\begin{array}{c}T_{e f f}^{2} \\
(\mathrm{~K})\end{array}$ & $\begin{array}{c}T_{e f f}{ }^{3} \\
(\mathrm{~K})\end{array}$ & $\begin{array}{c}T_{e f f} \text { Diff } \\
(\mathrm{K})\end{array}$ \\
\hline Gl 134 & 1.116 & 3670 & 3700 & - & +30 \\
\hline Gl 390 & 1.120 & 3665 & 3700 & - & +35 \\
\hline Gl 821 & 1.120 & 3665 & 3545 & - & -120 \\
\hline Gl 895 & 1.121 & 3664 & 3764 & - & +100 \\
\hline Gl 526 & 1.124 & 3662 & - & 3618 & -44 \\
\hline Gl 880 & 1.124 & 3662 & - & 3713 & +51 \\
\hline Gl 880 & 1.126 & 3660 & 3720 & - & +60 \\
\hline Gl $15 \mathrm{~A}$ & 1.130 & 3656 & 3603 & 3594 & -53 \\
\hline Gl 908 & 1.133 & 3653 & 3646 & - & -7 \\
\hline GJ 3098 & 1.139 & 3647 & 3641 & - & -6 \\
\hline Gl 87 & 1.141 & 3645 & 3638 & - & -7 \\
\hline Gl 526 & 1.147 & 3639 & 3649 & - & +10 \\
\hline Gl 289 & 1.154 & 3632 & 3576 & - & -56 \\
\hline $\mathrm{PM} 02441+4913$ & 1.160 & 3626 & 3685 & - & +59 \\
\hline Gl 507.1 & 1.171 & 3615 & 3650 & - & +35 \\
\hline Gl 411 & 1.184 & 3602 & 3563 & 3465 & -39 \\
\hline Gl 806 & 1.202 & 3584 & 3542 & - & -42 \\
\hline Gl 361 & 1.204 & 3582 & 3500 & - & -82 \\
\hline Gl 382 & 1.206 & 3580 & 3623 & - & +43 \\
\hline Gl 173 & 1.212 & 3575 & 3671 & - & +96 \\
\hline Gl 104 & 1.217 & 3571 & 3542 & - & -29 \\
\hline Gl $745 \mathrm{~B}$ & 1.217 & 3571 & 3494 & - & -77 \\
\hline $\mathrm{Gl} 745 \mathrm{~A}$ & 1.226 & 3563 & 3500 & - & -63 \\
\hline GJ 1009 & 1.232 & 3558 & 3600 & - & +42 \\
\hline GJ 2066 & 1.234 & 3556 & 3500 & - & -56 \\
\hline Gl 393 & 1.235 & 3555 & 3548 & - & -7 \\
\hline Gl 250B & 1.239 & 3552 & 3481 & - & -71 \\
\hline Gl 180 & 1.240 & 3551 & 3506 & - & -45 \\
\hline Gl 625 & 1.241 & 3550 & 3475 & - & -75 \\
\hline PM 09437-1747 & 1.246 & 3546 & 3302 & - & -244 \\
\hline Gl 176 & 1.250 & 3542 & 3680 & - & +138 \\
\hline PM $18007+2933$ & 1.251 & 3541 & 3509 & - & -32 \\
\hline Gl 70 & 1.299 & 3499 & 3458 & - & -41 \\
\hline Gl 297.2B & 1.304 & 3496 & 3544 & - & +48 \\
\hline LPM $0355+5214$ & 1.318 & 3486 & 3435 & - & -51 \\
\hline Gl 549B & 1.319 & 3486 & 3479 & - & -7 \\
\hline Gl 694 & 1.324 & 3482 & 3464 & - & -18 \\
\hline PM $20167+5017$ & 1.328 & 3480 & 3559 & - & +79 \\
\hline Gl $752 \mathrm{~A}$ & 1.333 & 3476 & 3558 & - & +82 \\
\hline Gl 671 & 1.338 & 3473 & 3433 & - & -40 \\
\hline Gl $615.2 \mathrm{C}$ & 1.339 & 3472 & 3454 & - & -18 \\
\hline PM $15118+3933$ & 1.340 & 3471 & 3435 & - & -36 \\
\hline Gl 436 & 1.351 & 3464 & - & 3416 & -48 \\
\hline Gl 811.1 & 1.352 & 3463 & 3473 & - & +10 \\
\hline PM 05557-2651 & 1.362 & 3457 & 3652 & - & +195 \\
\hline Gl $725 \mathrm{~A}$ & 1.364 & 3455 & 3441 & - & -14 \\
\hline GJ 3195 & 1.370 & 3451 & 3500 & - & +49 \\
\hline PM $18046+1354$ & 1.384 & 3441 & 3375 & - & -66 \\
\hline Gl $725 \mathrm{~A}$ & 1.390 & 3437 & - & 3407 & -30 \\
\hline GJ 4070 & 1.391 & 3436 & 3400 & - & -36 \\
\hline Gl 109 & 1.395 & 3434 & 3405 & - & -29 \\
\hline Gl 436 & 1.400 & 3431 & 3479 & - & +48 \\
\hline Gl $512 \mathrm{~A}$ & 1.403 & 3428 & 3498 & - & +70 \\
\hline Gl 581 & 1.409 & 3423 & - & 3442 & +19 \\
\hline Gl 687 & 1.410 & 3422 & - & 3413 & -9 \\
\hline Gl 687 & 1.416 & 3417 & 3439 & - & +22 \\
\hline GJ 1097 & 1.421 & 3413 & 3448 & - & +35 \\
\hline Gl 581 & 1.424 & 3410 & 3395 & - & -15 \\
\hline GJ 3459 & 1.425 & 3409 & 3358 & - & -51 \\
\hline PM $02534+1724$ & 1.429 & 3406 & 3377 & - & -29 \\
\hline Gl $725 \mathrm{~B}$ & 1.43 & 3405 & - & 3104 & -301 \\
\hline PM 06077-2544 & 1.433 & 3403 & 3356 & - & -47 \\
\hline GJ 1236 & 1.433 & 3403 & 3335 & - & -68 \\
\hline GJ 4040 & 1.437 & 3399 & 3470 & - & +71 \\
\hline Gl 849 & 1.438 & 3398 & 3530 & - & +132 \\
\hline Gl 388 & 1.452 & 3386 & 3370 & - & -16 \\
\hline Gl 251 & 1.456 & 3383 & 3448 & - & +65 \\
\hline GJ 3388 & 1.464 & 3376 & 3326 & - & -50 \\
\hline Gl $725 \mathrm{~B}$ & 1.474 & 3367 & 3345 & - & -22 \\
\hline GJ 3992 & 1.478 & 3364 & 3432 & - & +68 \\
\hline
\end{tabular}


Table 1:: continued.

\begin{tabular}{|c|c|c|c|c|c|}
\hline Star & $\begin{array}{c}(\mathrm{R}-\mathrm{I})_{c} \\
(\mathrm{mag})\end{array}$ & $\begin{array}{c}T_{e f f}{ }^{1} \\
(\mathrm{~K})\end{array}$ & $\begin{array}{c}T_{e f f}^{2} \\
(\mathrm{~K})\end{array}$ & $\begin{array}{c}T_{e f f}{ }^{3} \\
(\mathrm{~K})\end{array}$ & $\begin{array}{c}T_{e f f} \text { Diff } \\
\quad(\mathrm{K})\end{array}$ \\
\hline PM 09553-2715 & 1.480 & 3362 & 3346 & - & -16 \\
\hline GJ 3325 & 1.480 & 3362 & 3365 & - & +3 \\
\hline Gl 480 & 1.487 & 3356 & 3463 & - & +107 \\
\hline Gl 403 & 1.491 & 3353 & 3298 & - & -55 \\
\hline Gl 643 & 1.491 & 3353 & 3279 & - & -74 \\
\hline $\mathrm{PM} 00118+2259$ & 1.527 & 3322 & 3359 & - & +37 \\
\hline Gl 545 & 1.539 & 3311 & 3341 & - & +30 \\
\hline Gl 896A & 1.546 & 3305 & 3353 & - & +48 \\
\hline Gl 628 & 1.561 & 3292 & 3272 & - & -20 \\
\hline PM $15354+6005$ & 1.572 & 3283 & 3252 & - & -31 \\
\hline Gl 876 & 1.574 & 3282 & 3247 & - & -35 \\
\hline GJ 3378 & 1.575 & 3281 & 3340 & - & +59 \\
\hline GJ 1148 & 1.582 & 3274 & 3304 & - & +30 \\
\hline Gl 273 & 1.585 & 3272 & 3317 & - & +45 \\
\hline Gl 699 & 1.585 & 3272 & 3228 & - & -44 \\
\hline Gl 699 & 1.592 & 3266 & - & 3224 & -42 \\
\hline Gl $15 B$ & 1.595 & 3263 & 3218 & - & -45 \\
\hline GJ 3707 & 1.599 & 3260 & 3385 & - & +125 \\
\hline GJ 4065 & 1.599 & 3260 & 3223 & - & -37 \\
\hline $\mathrm{Gl} 118.2 \mathrm{C}$ & 1.600 & 3259 & 3227 & - & -32 \\
\hline Gl $105 \mathrm{~B}$ & 1.607 & 3254 & 3284 & - & +30 \\
\hline GJ 1207 & 1.608 & 3254 & 3229 & - & -25 \\
\hline Gl 729 & 1.610 & 3252 & 3240 & - & -12 \\
\hline Gl 402 & 1.616 & 3248 & 3238 & - & -10 \\
\hline GJ 3150 & 1.617 & 3248 & 3216 & - & -32 \\
\hline GJ 4269 & 1.622 & 3244 & 3226 & - & -18 \\
\hline GJ 4333 & 1.623 & 3244 & 3324 & - & +80 \\
\hline PM 19321-1119 & 1.626 & 3242 & 3211 & - & -31 \\
\hline GJ 1129 & 1.631 & 3239 & 3243 & - & +4 \\
\hline GJ 3379 & 1.633 & 3237 & 3214 & - & -23 \\
\hline Gl $783.2 \mathrm{~B}$ & 1.633 & 3237 & 3217 & - & -20 \\
\hline GJ 4367 & 1.633 & 3237 & 3221 & - & -16 \\
\hline GJ 1092 & 1.639 & 3233 & 3207 & - & -26 \\
\hline Gl 213 & 1.643 & 3231 & 3250 & - & +19 \\
\hline PM 20525-1658 & 1.643 & 3231 & 3205 & - & -26 \\
\hline Gl 555 & 1.648 & 3227 & 3211 & - & -16 \\
\hline Gl 544B & 1.659 & 3220 & 3191 & - & -29 \\
\hline Gl 324B & 1.660 & 3219 & 3166 & - & -53 \\
\hline GJ 3991 & 1.663 & 3217 & 3284 & - & +67 \\
\hline GJ 3105 & 1.674 & 3210 & 3269 & - & +59 \\
\hline Gl 53.1B & 1.675 & 3210 & 3232 & - & +22 \\
\hline PM $18453+1851$ & 1.675 & 3210 & 3214 & - & +4 \\
\hline GJ 3142 & 1.677 & 3208 & 3257 & - & +49 \\
\hline GJ 1289 & 1.677 & 3208 & 3173 & - & -35 \\
\hline Gl 232 & 1.679 & 3207 & 3165 & - & -42 \\
\hline Gl 447 & 1.697 & 3198 & 3192 & - & -6 \\
\hline Gl 102 & 1.698 & 3194 & 3199 & - & +5 \\
\hline GJ 3323 & 1.706 & 3189 & 3143 & - & -46 \\
\hline Gl 166C & 1.726 & 3176 & 3167 & - & -9 \\
\hline Gl 585 & 1.737 & 3169 & 3164 & - & -5 \\
\hline GJ $1230 \mathrm{~A}$ & 1.740 & 3167 & 3232 & - & +65 \\
\hline GJ 1151 & 1.761 & 3153 & 3118 & - & -35 \\
\hline GJ 3668 & 1.781 & 3140 & 3109 & - & -31 \\
\hline PM $03361+3118$ & 1.787 & 3136 & 3086 & - & -50 \\
\hline GJ 3253 & 1.787 & 3136 & 3079 & - & -57 \\
\hline Gl $777 \mathrm{~B}$ & 1.788 & 3135 & 3144 & - & +9 \\
\hline GJ 1288 & 1.794 & 3131 & 3113 & - & -18 \\
\hline Gl 54.1 & 1.796 & 3130 & 3056 & - & -74 \\
\hline GJ 3976 & 1.835 & 3096 & 3054 & - & -42 \\
\hline GJ 1253 & 1.837 & 3094 & 3045 & - & -49 \\
\hline Gl 83.1 & 1.849 & 3084 & 3080 & - & -4 \\
\hline GJ 3631 & 1.873 & 3063 & 3039 & - & -24 \\
\hline Gl $896 \mathrm{~B}$ & 1.873 & 3063 & 3072 & - & +9 \\
\hline GJ 3119 & 1.875 & 3061 & 3011 & - & -50 \\
\hline GJ 3380 & 1.902 & 3038 & 3000 & - & -38 \\
\hline GJ 1029 & 1.936 & 3008 & 2981 & - & -27 \\
\hline PM 10430-0912 & 1.963 & 2985 & 2951 & - & -34 \\
\hline Gl 551 & 2.03 & 2927 & - & 3054 & +127 \\
\hline Gl 905 & 2.031 & 3926 & 2930 & - & +4 \\
\hline GJ $1245 B$ & 2.095 & 2898 & 2859 & - & -39 \\
\hline
\end{tabular}


Table 1:: continued.

\begin{tabular}{|lccccc|}
\hline Star & $\begin{array}{c}(\mathrm{R}-\mathrm{I})_{c} \\
(\mathrm{mag})\end{array}$ & $\begin{array}{c}T_{\text {eff }}{ }^{1} \\
(\mathrm{~K})\end{array}$ & $\begin{array}{c}T_{\text {eff }}{ }^{2} \\
(\mathrm{~K})\end{array}$ & $\begin{array}{c}T_{\text {eff }}{ }^{3} \\
(\mathrm{~K})\end{array}$ & $\begin{array}{c}T_{\text {eff }} \text { Diff } \\
\text { (K) }\end{array}$ \\
GJ 3147 & & & & & -15 \\
PM 00115+5908 & 2.102 & 2895 & 2880 & - & -23 \\
Gl 412B & 2.121 & 2887 & 2864 & - & -9 \\
GJ 3146 & 2.157 & 2872 & 2863 & - & -39 \\
Gl 406 & 2.165 & 2868 & 2829 & - & -16 \\
GJ 1111 & 2.246 & 2834 & 2818 & - & +9 \\
PM 02530+1652 & 2.363 & 2791 & 2800 & - & +24 \\
Gl 644C & 2.404 & 2776 & 2800 & - & +59 \\
& 2.450 & 2759 & 2700 & - & + \\
\hline
\end{tabular}

${ }^{1}$ Effective temperature from the $(\mathrm{R}-\mathrm{I})_{C}$ color $/ T_{\text {eff }}$ calibration

${ }^{2}$ Effective temperature from Mann et al. 2015

${ }^{3}$ Effective temperature from Boyajian et al. 2012 
Table 2:: Parameters of our sample of mid-K, late-K stars, M0-M1, M2, M3, M4,

M5, M6 and M7 dwarfs. We also indicate the number of high resolution spectra for

the stars that have observed.

\begin{tabular}{|c|c|c|c|c|c|c|c|c|c|c|c|c|}
\hline Star & $\underset{(\mathrm{mag})}{\mathrm{V}}$ & $\begin{array}{c}(\mathrm{R}-\mathrm{I})_{C} \\
(\mathrm{mag})\end{array}$ & $\begin{array}{c}T_{e f f}{ }^{1} \\
(\mathrm{~K})\end{array}$ & $\begin{array}{c}T_{e f f}{ }^{2} \\
(\mathrm{~K})\end{array}$ & $\begin{array}{c}T_{e f f} \pm 3 \sigma^{3} \\
(\mathrm{~K})\end{array}$ & $\begin{array}{l}\text { Spect. } \\
\text { Type }\end{array}$ & $\begin{array}{c}\pi \\
(\mathrm{m} ")\end{array}$ & $\begin{array}{c}M_{V} \\
\text { (mag) }\end{array}$ & $\begin{array}{c}R_{\star} \\
\left(R_{\odot}\right)\end{array}$ & {$[\mathrm{M} / \mathrm{H}]$} & $\begin{array}{l}\text { Hi. Res. } \\
\text { Spectra }\end{array}$ & Comments \\
\hline & & & & & Mid-K sampl & & & & & & & \\
\hline GJ 1139 & 9.567 & 0.656 & 4366 & 4391 & $4378 \pm 13$ & dK5.4 & $43.4531 \pm 0.0293$ & $7.757 \pm 0.021$ & $0.592 \pm 0.016$ & -0.33 & & \\
\hline GJ 1176 & 9.225 & 0.620 & 4456 & 4509 & $4482 \pm 27$ & $\mathrm{dK} 4.3$ & $40.6674 \pm 0.0433$ & $7.271 \pm 0.022$ & $0.686 \pm 0.025$ & +0.07 & 35 & \\
\hline GJ 1189 & 9.30 & 0.566 & 4591 & 4577 & $4584 \pm 7$ & dK 3.6 & $30.6458 \pm 0.0552$ & $6.732 \pm 0.024$ & $0.813 \pm 0.018$ & +0.04 & & Binary? \\
\hline GJ 1190 & 9.839 & 0.644 & 4396 & 4333 & $4364 \pm 31$ & sdK5.4 & $38.8659 \pm 0.0589$ & $7.787 \pm 0.023$ & $0.591 \pm 0.024$ & -0.95 & 5 & \\
\hline GJ 1257 & 9.811 & 0.694 & 4270 & 4310 & $4340 \pm 30$ & dK5.6 & $41.3937 \pm 0.0643$ & $7.896 \pm 0.023$ & $0.572 \pm 0.023$ & -0.23 & & Subdwarf? \\
\hline GJ 1259 & 8.770 & 0.674 & 4321 & 4398 & $4359 \pm 39$ & dK5.5 & $41.5127 \pm 0.0553$ & $6.861 \pm 0.023$ & $0.908 \pm 0.043$ & +0.01 & 2 & Binary? \\
\hline GJ 2018 & 9.956 & 0.572 & 4576 & 4563 & $4569 \pm 6$ & dK3.6 & $27.5963 \pm 0.0422$ & $7.160 \pm 0.023$ & $0.675 \pm 0.014$ & -0.17 & 2 & \\
\hline GJ 2096 & 9.10 & 0.638 & 4411 & 4328 & $4369 \pm 41$ & dK5.4 & $38.1457 \pm 0.7610$ & $7.007 \pm 0.063$ & $0.842 \pm 0.058$ & -0.73 & & Binary? \\
\hline GJ 3012 & 9.475 & 0.692 & 4275 & - & $4275 \pm 35$ & dK6.0 & $35.6385 \pm 0.4414$ & $7.235 \pm 0.047$ & $0.815 \pm 0.046$ & & & Binary? \\
\hline GJ 3065A & 10.23 & 0.609 & 4484 & 4521 & $4503 \pm 18$ & dK4.0 & $32.1455 \pm 0.0589$ & $7.766 \pm 0.024$ & $0.538 \pm 0.017$ & +0.00 & & Subdwarf? \\
\hline GJ 3065B & 10.23 & 0.609 & 4484 & 4521 & $4503 \pm 18$ & $\mathrm{dK} 4.0$ & $32.1455 \pm 0.0589$ & $7.766 \pm 0.024$ & $0.538 \pm 0.017$ & +0.00 & & Subdwarf? \\
\hline GJ 3066 & 9.10 & 0.572 & 4576 & - & $4576 \pm 35$ & dK3.6 & $39.8944 \pm 0.0298$ & $7.105 \pm 0.022$ & $0.689 \pm 0.030$ & & & \\
\hline GJ 3070 & 11.87 & 0.682 & 4301 & - & $4301 \pm 35$ & dK5.8 & $15.8500 \pm 0.0433$ & $7.870 \pm 0.026$ & $0.596 \pm 0.028$ & -0.23 & & \\
\hline GJ 3089 & 10.540 & 0.597 & 4514 & - & $4514 \pm 35$ & $\mathrm{dK} 4.0$ & $21.4901 \pm 0.0328$ & $7.201 \pm 0.023$ & $0.691 \pm 0.031$ & -0.17 & & \\
\hline GJ 3415 & 9.145 & 0.608 & 4486 & - & $4486 \pm 35$ & $\mathrm{dK} 4.3$ & $39.7680 \pm 0.0680$ & $7.143 \pm 0.024$ & $0.725 \pm 0.032$ & & & \\
\hline GJ 3498 & 10.06 & 0.596 & 4516 & - & $4516 \pm 35$ & dK3.9 & $27.7685 \pm 0.0440$ & $7.278 \pm 0.023$ & $0.666 \pm 0.030$ & & & \\
\hline GJ 3606 & 8.67 & 0.596 & 4516 & - & $4516 \pm 35$ & dK3.9 & $42.4131 \pm 0.0403$ & $6.808 \pm 0.022$ & $0.827 \pm 0.036$ & & & Binary? \\
\hline GJ 3905 & 8.868 & 0.560 & 4606 & 4677 & $4641 \pm 36$ & dK3.2 & $45.1281 \pm 0.0525$ & $7.140 \pm 0.023$ & $0.644 \pm 0.028$ & -0.34 & 16 & \\
\hline GJ $3922 \mathrm{~A}$ & 10.088 & 0.621 & 4454 & 4346 & $4400 \pm 54$ & dK5.2 & $38.9014 \pm 0.2210$ & $8.038 \pm 0.032$ & $0.512 \pm 0.033$ & +0.29 & & Subdwarf? \\
\hline GJ 3922B & 10.088 & 0.621 & 4454 & 4346 & $4400 \pm 54$ & dK5.2 & $38.9014 \pm 0.2210$ & $8.038 \pm 0.032$ & $0.512 \pm 0.033$ & +0.29 & & Subdwarf? \\
\hline GJ 3948 & 9.663 & 0.600 & 4506 & - & $4506 \pm 35$ & $\mathrm{dK} 4.1$ & $33.6296 \pm 0.0201$ & $7.297 \pm 0.021$ & $0.666 \pm 0.029$ & & & \\
\hline GJ 4061 & 9.318 & 0.627 & 4440 & 4428 & $4434 \pm 6$ & dK5.0 & $39.6956 \pm 0.0255$ & $7.312 \pm 0.021$ & $0.697 \pm 0.014$ & -0.12 & & \\
\hline GJ 4280 & 9.512 & 0.609 & 4484 & 4465 & $4475 \pm 10$ & $\mathrm{dK} 4.4$ & $38.3161 \pm 0.0254$ & $7.429 \pm 0.021$ & $0.641 \pm 0.015$ & +0.17 & & \\
\hline GJ 4301 & 9.784 & 0.540 & 4693 & 4386 & $4540 \pm 153$ & dK3.8 & $35.7555 \pm 0.0477$ & $7.551 \pm 0.023$ & $0.577 \pm 0.084$ & +0.30 & & \\
\hline GJ 4322B & 9.04 & 0.578 & 4561 & 4630 & $4595 \pm 35$ & $\mathrm{dK} 3.5$ & $35.1337 \pm 0.0249$ & $6.769 \pm 0.022$ & $0.792 \pm 0.034$ & +0.13 & 8 & \\
\hline GJ 4328 & 9.720 & 0.680 & 4306 & - & $4306 \pm 35$ & dK5.8 & $28.2434 \pm 0.1088$ & $6.975 \pm 0.028$ & $0.897 \pm 0.043$ & & & Binary? \\
\hline GJ 9036 & 9.790 & 0.621 & 4454 & 4469 & $4462 \pm 8$ & dK4.6 & $37.0209 \pm 0.0398$ & $7.632 \pm 0.022$ & $0.589 \pm 0.013$ & -0.12 & 5 & \\
\hline GJ 9356 & 9.765 & 0.608 & 4486 & 4539 & $4512 \pm 27$ & $\mathrm{dK} 4.0$ & $30.0976 \pm 0.0702$ & $7.158 \pm 0.025$ & $0.706 \pm 0.027$ & -0.12 & 32 & \\
\hline GJ 9401 & 10.774 & 0.585 & 4544 & 4530 & $4537 \pm 7$ & dK3.8 & $17.4833 \pm 0.0292$ & $6.987 \pm 0.024$ & $0.749 \pm 0.016$ & -0.10 & & \\
\hline GJ 9427 & 9.340 & 0.632 & 4426 & - & $4426 \pm 35$ & dK5.1 & $40.3147 \pm 0.0368$ & $7.367 \pm 0.022$ & $0.684 \pm 0.030$ & & & \\
\hline GJ $9452 \mathrm{~A}$ & 10.181 & 0.549 & 4654 & 4368 & $4511 \pm 143$ & dK4.0 & $28.1847 \pm 0.0206$ & $7.431 \pm 0.022$ & $0.623 \pm 0.086$ & -0.59 & & \\
\hline Gl 3 & 8.485 & 0.555 & 4628 & 4572 & $4600 \pm 28$ & sdK3.5 & $61.0313 \pm 0.0325$ & $7.413 \pm 0.021$ & $0.587 \pm 0.022$ & -0.60 & 3 & \\
\hline Gl 30 & 8.725 & 0.608 & 4486 & 4450 & $4468 \pm 18$ & $\mathrm{dK} 4.6$ & $47.4811 \pm 0.1021$ & $7.108 \pm 0.025$ & $0.747 \pm 0.023$ & -0.02 & & \\
\hline Gl $32 \mathrm{~A}$ & 7.880 & 0.655 & 4369 & 4392 & $4380 \pm 11$ & dK5.3 & $63.5070 \pm 0.0430$ & $6.894 \pm 0.021$ & $0.880 \pm 0.022$ & & 1 & Binary? \\
\hline Gl 39 & 9.216 & 0.668 & 4336 & 4287 & $4311 \pm 24$ & dK5.8 & $44.9546 \pm 0.0530$ & $7.480 \pm 0.023$ & $0.708 \pm 0.025$ & +0.11 & 2 & \\
\hline Gl 50 & 10.492 & 0.694 & 4270 & 4344 & $4307 \pm 37$ & $\mathrm{dK} 5.8$ & $29.0239 \pm 0.0353$ & $7.806 \pm 0.023$ & $0.611 \pm 0.028$ & +0.56 & 4 & \\
\hline Gl $53.1 \mathrm{~A}$ & 8.407 & 0.596 & 4516 & 4565 & $4541 \pm 24$ & dK3.8 & $48.6871 \pm 0.0693$ & $6.844 \pm 0.023$ & $0.798 \pm 0.028$ & +0.16 & 2 & \\
\hline Gl 65.2 & 9.88 & 0.568 & 4586 & 4523 & $4554 \pm 62$ & dK3.7 & $28.4645 \pm 0.0285$ & $7.152 \pm 0.022$ & $0.686 \pm 0.045$ & -0.12 & 6 & \\
\hline Gl 69 & 8.417 & 0.656 & 4366 & 4325 & $4345 \pm 20$ & dK5.5 & $73.9913 \pm 0.0353$ & $7.763 \pm 0.021$ & $0.606 \pm 0.019$ & -0.24 & 1 & \\
\hline Gl 81.2 & 8.608 & 0.555 & 4628 & 4597 & $4612 \pm 15$ & $\mathrm{dK} 3.4$ & $49.7428 \pm 0.0334$ & $7.092 \pm 0.021$ & $0.674 \pm 0.018$ & +0.08 & & \\
\hline Gl 100A & 8.767 & 0.625 & 4444 & 4448 & $4446 \pm 2$ & dK4.9 & $50.8621 \pm 0.0542$ & $7.299 \pm 0.022$ & $0.695 \pm 0.012$ & -0.28 & & \\
\hline Gl 105.5 & 9.509 & 0.644 & 4396 & 4340 & $4368 \pm 28$ & dK5.4 & $41.7670 \pm 0.0403$ & $7.613 \pm 0.022$ & $0.638 \pm 0.025$ & -0.01 & 1 & \\
\hline Gl 106 & 8.210 & 0.566 & 4591 & - & $4591 \pm 35$ & dK3.5 & $53.5579 \pm 0.1814$ & $6.854 \pm 0.027$ & $0.764 \pm 0.035$ & & & \\
\hline Gl 112.1 & 8.574 & 0.656 & 4366 & 4373 & $4369 \pm 4$ & $\mathrm{dK} 5.4$ & $61.9128 \pm 0.0461$ & $7.533 \pm 0.022$ & $0.661 \pm 0.013$ & & & \\
\hline GL 114 & 8.873 & 0.578 & 4561 & 4504 & $4532 \pm 28$ & dK3.9 & $42.7647 \pm 0.0576$ & $7.028 \pm 0.023$ & $0.738 \pm 0.028$ & +0.17 & & \\
\hline Gl 131 & 9.141 & 0.651 & 4379 & 4330 & $4354 \pm 24$ & dK5.5 & $53.5696 \pm 0.0410$ & $7.786 \pm 0.022$ & $0.595 \pm 0.021$ & & 9 & \\
\hline Gl $140.1 \mathrm{~A}$ & 8.68 & 0.62 & 4456 & 4459 & $4457 \pm 2$ & dK4.7 & $51.8133 \pm 0.0279$ & $7.252 \pm 0.021$ & $0.705 \pm 0.012$ & -0.20 & 46 & \\
\hline
\end{tabular}


Table 2:: continued.

\begin{tabular}{|c|c|c|c|c|c|c|c|c|c|c|c|c|}
\hline Star & $\begin{array}{c}\mathrm{V} \\
(\mathrm{mag})\end{array}$ & $\begin{array}{c}(\mathrm{R}-\mathrm{I})_{C} \\
(\mathrm{mag})\end{array}$ & $\begin{array}{c}T_{e f f}{ }^{1} \\
(\mathrm{~K})\end{array}$ & $\begin{array}{c}T_{e f f}{ }^{2} \\
(\mathrm{~K})\end{array}$ & $T_{\text {eff }} \pm 3 \sigma^{3}$ & $\begin{array}{l}\text { Spect. } \\
\text { Type }\end{array}$ & $\begin{array}{c}\pi \\
(\mathrm{m} ")\end{array}$ & $\begin{array}{c}M_{V} \\
(\mathrm{mag})\end{array}$ & $\begin{array}{c}R_{\star} \\
\left(R_{\odot}\right)\end{array}$ & {$[\mathrm{M} / \mathrm{H}]$} & $\begin{array}{l}\text { Hi. Res. } \\
\text { Spectra }\end{array}$ & Comments \\
\hline Gl $140.1 \mathrm{~B}$ & 10.02 & 0.62 & 4456 & 4459 & $4457 \pm 2$ & $\mathrm{dK} ?$ & $51.8098 \pm 0.0232$ & $8.592 \pm 0.021$ & $0.380 \pm 0.006$ & -0.20 & 46 & \\
\hline Gl 143 & 8.143 & 0.564 & 4596 & 4574 & $4585 \pm 11$ & dK3.6 & $61.2455 \pm 0.0264$ & $7.078 \pm 0.021$ & $0.692 \pm 0.016$ & +0.03 & 54 & \\
\hline Gl 156.2 & 8.255 & 0.680 & 4306 & 4419 & $4362 \pm 57$ & dK5.4 & $61.6177 \pm 0.0262$ & $7.204 \pm 0.021$ & $0.774 \pm 0.047$ & +0.02 & & \\
\hline Gl 160.2 & 9.675 & 0.611 & 4479 & - & $4479 \pm 35$ & $\mathrm{dK} 4.4$ & $38.6473 \pm 0.0582$ & $7.611 \pm 0.023$ & $0.588 \pm 0.026$ & & 94 & \\
\hline Gl 161.2 & 10.90 & 0.617 & 4464 & 4578 & $4521 \pm 57$ & dK3.9 & $20.9946 \pm 0.1760$ & $7.511 \pm 0.038$ & $0.596 \pm 0.042$ & -0.32 & & \\
\hline Gl 162.2 & 9.715 & 0.651 & 4379 & 4355 & $4367 \pm 12$ & dK5.4 & $36.5894 \pm 0.0213$ & $7.537 \pm 0.021$ & $0.661 \pm 0.014$ & & 15 & \\
\hline Gl 167 & 7.634 & 0.557 & 4619 & 4569 & $4594 \pm 25$ & dK3.5 & $76.5847 \pm 0.0304$ & $7.055 \pm 0.021$ & $0.695 \pm 0.024$ & +0.06 & 1 & \\
\hline Gl $173.1 \mathrm{~A}$ & 9.195 & 0.561 & 4603 & - & $4603 \pm 35$ & $\mathrm{dK} 3.4$ & $36.8039 \pm 0.3853$ & $7.024 \pm 0.043$ & $0.700 \pm 0.037$ & -0.33 & 1 & Binary? \\
\hline Gl 200A & 7.86 & 0.58 & 4556 & 4641 & $4598 \pm 43$ & dK3.5 & $63.6514 \pm 0.0476$ & $6.879 \pm 0.022$ & $0.751 \pm 0.037$ & -0.30 & 1 & \\
\hline Gl 204 & 7.642 & 0.590 & 4531 & 4550 & $4540 \pm 10$ & $\mathrm{dK} 3.8$ & $77.3049 \pm 0.0387$ & $7.083 \pm 0.021$ & $0.715 \pm 0.016$ & -0.09 & 261 & \\
\hline Gl 223.1 & 10.715 & 0.609 & 4484 & 4452 & $4468 \pm 16$ & $\mathrm{dK} 4.6$ & $26.3699 \pm 0.0343$ & $7.821 \pm 0.023$ & $0.538 \pm 0.015$ & -0.66 & 12 & \\
\hline Gl 247 & 8.58 & 0.656 & 4366 & 4288 & $4327 \pm 39$ & dK5.7 & $59.1275 \pm 0.0659$ & $7.439 \pm 0.022$ & $0.713 \pm 0.034$ & +0.03 & & \\
\hline Gl 256 & 9.157 & 0.642 & 4401 & 4402 & $4401 \pm 1$ & $\mathrm{dK} 5.2$ & $44.5236 \pm 0.0363$ & $7.400 \pm 0.022$ & $0.686 \pm 0.018$ & & & \\
\hline Gl 264 & 8.686 & 0.629 & 4434 & 4428 & $4431 \pm 3$ & dK5.0 & $57.7938 \pm 0.3355$ & $7.495 \pm 0.033$ & $0.642 \pm 0.015$ & -0.20 & 1 & \\
\hline Gl 266 & 9.845 & 0.673 & 4323 & 4330 & $4326 \pm 4$ & dK5.7 & $38.2618 \pm 0.2672$ & $7.759 \pm 0.035$ & $0.616 \pm 0.016$ & +0.17 & 7 & \\
\hline Gl 268.2 & 9.079 & 0.662 & 4351 & 4368 & $4359 \pm 9$ & dK5.5 & $50.9662 \pm 0.0306$ & $7.615 \pm 0.021$ & $0.641 \pm 0.015$ & +0.12 & 11 & \\
\hline Gl 291.1 & 10.035 & 0.622 & 4451 & 4467 & $4459 \pm 8$ & $\mathrm{dK} 4.7$ & $30.7749 \pm 0.0204$ & $7.476 \pm 0.021$ & $0.635 \pm 0.014$ & -0.23 & 15 & \\
\hline Gl 301.1 & 8.84 & 0.584 & 4546 & 4466 & $4506 \pm 40$ & $\mathrm{dK} 4.0$ & $43.7398 \pm 0.0515$ & $7.044 \pm 0.023$ & $0.748 \pm 0.036$ & -0.40 & & \\
\hline Gl 308.2 & 11.950 & 0.537 & 4706 & 4756 & $4731 \pm 25$ & $\operatorname{sdK} 2.8$ & $12.0109 \pm 0.0445$ & $7.348 \pm 0.028$ & $0.547 \pm 0.020$ & -1.25 & & \\
\hline Gl 308.3 & 10.795 & 0.634 & 4421 & 4377 & $4399 \pm 22$ & dK5.2 & $24.5923 \pm 0.0274$ & $7.749 \pm 0.022$ & $0.585 \pm 0.020$ & +0.37 & 11 & \\
\hline Gl 330.1 & 9.214 & 0.656 & 4366 & - & $4366 \pm 35$ & sdK5.4 & $47.5479 \pm 0.0502$ & $7.600 \pm 0.022$ & $0.643 \pm 0.028$ & -0.60 & & \\
\hline Gl 339 & 7.925 & 0.575 & 4569 & 4641 & $4605 \pm 36$ & $\mathrm{dK} 3.4$ & $56.1805 \pm 0.0550$ & $6.673 \pm 0.022$ & $0.822 \pm 0.036$ & -0.07 & & Binary? \\
\hline Gl 340.3 & 9.082 & 0.568 & 4586 & 4554 & $4570 \pm 16$ & dK3.6 & $41.3649 \pm 0.0425$ & $7.165 \pm 0.022$ & $0.673 \pm 0.019$ & +0.09 & 22 & \\
\hline Gl 341.1 & 9.300 & 0.632 & 4426 & 4338 & $4382 \pm 44$ & dK5.3 & $43.2985 \pm 0.0338$ & $7.482 \pm 0.022$ & $0.670 \pm 0.034$ & +0.07 & 2 & \\
\hline Gl 342 & 9.045 & 0.632 & 4426 & 4542 & $4484 \pm 58$ & $\mathrm{dK} 4.3$ & $47.7356 \pm 0.0227$ & $7.439 \pm 0.021$ & $0.634 \pm 0.038$ & +0.16 & 4 & \\
\hline Gl 365 & 8.154 & 0.584 & 4546 & 4640 & $4593 \pm 47$ & $\mathrm{dK} 3.5$ & $55.4519 \pm 0.1298$ & $6.874 \pm 0.025$ & $0.756 \pm 0.041$ & +0.34 & & \\
\hline Gl 370 & 7.651 & 0.622 & 4451 & 4496 & $4473 \pm 23$ & dK4.5 & $88.6158 \pm 0.0406$ & $7.389 \pm 0.021$ & $0.654 \pm 0.022$ & -0.22 & 383 & \\
\hline Gl 371 & 8.840 & 0.605 & 4494 & 4378 & $4436 \pm 58$ & dK5.0 & $48.6850 \pm 0.0813$ & $7.277 \pm 0.024$ & $0.708 \pm 0.044$ & +0.34 & & \\
\hline Gl 383.1 & 9.536 & 0.644 & 4396 & 4418 & $4407 \pm 11$ & sdK5.2 & $43.5298 \pm 0.0370$ & $7.730 \pm 0.022$ & $0.587 \pm 0.014$ & -0.78 & & \\
\hline Gl 389.1 & 9.995 & 0.635 & 4419 & - & $4419 \pm 35$ & dK5.1 & $34.3613 \pm 0.0623$ & $7.675 \pm 0.024$ & $0.597 \pm 0.026$ & & 22 & \\
\hline Gl 412.3 & 9.335 & 0.695 & 4268 & 4201 & $4234 \pm 33$ & $\mathrm{dK} 6.2$ & $36.8377 \pm 0.3025$ & $7.166 \pm 0.038$ & $0.869 \pm 0.048$ & & 6 & Binary? \\
\hline Gl 413 & 9.77 & 0.638 & 4411 & - & $4411 \pm 35$ & dK5.1 & $35.4832 \pm 0.0496$ & $7.520 \pm 0.023$ & $0.645 \pm 0.028$ & & & \\
\hline Gl 415 & 9.219 & 0.591 & 4529 & 4413 & $4471 \pm 58$ & $\operatorname{sdK} 4.5$ & $50.3340 \pm 0.0478$ & $7.728 \pm 0.022$ & $0.560 \pm 0.034$ & -1.17 & & \\
\hline Gl 416 & 9.038 & 0.684 & 4295 & 4276 & $4285 \pm 9$ & dK5.9 & $45.4075 \pm 0.5798$ & $7.324 \pm 0.048$ & $0.776 \pm 0.028$ & & & \\
\hline Gl 418 & 8.672 & 0.632 & 4426 & 4390 & $4408 \pm 18$ & dK5.2 & $54.4599 \pm 0.0620$ & $7.352 \pm 0.022$ & $0.698 \pm 0.021$ & +0.13 & 6 & \\
\hline Gl 435 & 7.762 & 0.582 & 4551 & 4609 & $4580 \pm 29$ & sdK3.6 & $78.2300 \pm 0.0694$ & $7.229 \pm 0.022$ & $0.648 \pm 0.025$ & -0.52 & 8 & \\
\hline Gl 435.1 & 9.585 & 0.670 & 4331 & 4460 & $4396 \pm 36$ & $\mathrm{dK} 5.2$ & $32.2570 \pm 0.2491$ & $7.128 \pm 0.037$ & $0.781 \pm 0.040$ & -0.20 & & Binary? \\
\hline Gl 439 & 8.992 & 0.584 & 4546 & 4559 & $4552 \pm 7$ & dK3.7 & $38.6381 \pm 0.0499$ & $6.927 \pm 0.023$ & $0.761 \pm 0.016$ & +0.05 & & \\
\hline Gl 453 & 6.964 & 0.584 & 4546 & 4553 & $4549 \pm 3$ & dK3.8 & $98.1908 \pm 0.0413$ & $6.924 \pm 0.021$ & $0.764 \pm 0.013$ & +0.20 & 2 & \\
\hline Gl 454.1 & 10.955 & 0.568 & 4586 & - & $4586 \pm 35$ & $\mathrm{sdK} 3.5$ & $19.7789 \pm 0.0461$ & $7.436 \pm 0.025$ & $0.587 \pm 0.026$ & & 11 & \\
\hline Gl 468 & 9.227 & 0.603 & 4499 & 4424 & $4461 \pm 37$ & $\mathrm{dK} 4.6$ & $41.9980 \pm 0.0542$ & $7.343 \pm 0.023$ & $0.674 \pm 0.030$ & & 10 & \\
\hline Gl 481 & 7.927 & 0.608 & 4486 & 4452 & $4469 \pm 17$ & $\mathrm{dK} 4.5$ & $70.8894 \pm 0.1099$ & $7.180 \pm 0.023$ & $0.722 \pm 0.021$ & -0.20 & 1 & \\
\hline Gl 488.2 & 10.425 & 0.609 & 4484 & 4561 & $4522 \pm 39$ & dK3.9 & $25.1861 \pm 0.0526$ & $7.431 \pm 0.025$ & $0.618 \pm 0.031$ & -0.30 & 4 & \\
\hline Gl 489 & 9.126 & 0.585 & 4544 & 4510 & $4527 \pm 17$ & dK3.9 & $39.0038 \pm 0.3925$ & $7.082 \pm 0.042$ & $0.723 \pm 0.028$ & -0.09 & & \\
\hline Gl 493 & 9.765 & 0.635 & 4419 & 4368 & $4393 \pm 25$ & $\mathrm{dK} 5.3$ & $36.6932 \pm 0.0631$ & $7.588 \pm 0.024$ & $0.633 \pm 0.023$ & & 3 & \\
\hline Gl 498 & 9.308 & 0.632 & 4426 & 4452 & $4439 \pm 13$ & sdK5.0 & $46.2990 \pm 0.0232$ & $7.636 \pm 0.021$ & $0.599 \pm 0.015$ & -2.16 & 5 & \\
\hline Gl 506.1 & 10.845 & 0.532 & 4728 & 4643 & $4685 \pm 42$ & sdK3.0 & $21.6874 \pm 0.0546$ & $7.526 \pm 0.025$ & $0.522 \pm 0.026$ & -1.07 & 6 & \\
\hline Gl 518.1 & 9.992 & 0.573 & 4574 & 4481 & $4528 \pm 46$ & sdK3.9 & $31.7726 \pm 0.0447$ & $7.502 \pm 0.023$ & $0.595 \pm 0.032$ & -0.75 & & \\
\hline Gl $528 \mathrm{~A}$ & 7.139 & 0.584 & 4546 & 4490 & $4518 \pm 28$ & dK3.9 & $73.9239 \pm 0.0653$ & $6.483 \pm 0.022$ & $0.959 \pm 0.037$ & -0.08 & & Binary? \\
\hline Gl 529 & 8.166 & 0.640 & 4406 & 4296 & $4351 \pm 56$ & dK5.5 & $71.1199 \pm 0.0843$ & $7.426 \pm 0.023$ & $0.704 \pm 0.043$ & +0.20 & 8 & \\
\hline Gl 535 & 9.029 & 0.608 & 4486 & 4460 & $4473 \pm 13$ & $\mathrm{dK} 4.5$ & $42.0153 \pm 0.0442$ & $7.146 \pm 0.022$ & $0.731 \pm 0.019$ & +0.06 & & \\
\hline Gl 566B & 6.816 & 0.608 & 4486 & 4350 & $4418 \pm 68$ & sdK5.1 & $148.2131 \pm 0.0464$ & $7.670 \pm 0.021$ & $0.598 \pm 0.041$ & & & Subdwarf? \\
\hline Gl $570 \mathrm{~A}$ & 5.730 & 0.555 & 4628 & 4611 & $4619 \pm 8$ & dK3.4 & $170.0112 \pm 0.0851$ & $6.882 \pm 0.021$ & $0.738 \pm 0.015$ & -0.04 & 38 & \\
\hline
\end{tabular}


Table 2:: continued.

\begin{tabular}{|c|c|c|c|c|c|c|c|c|c|c|c|c|}
\hline Star & $\begin{array}{c}\mathrm{V} \\
(\mathrm{mag})\end{array}$ & $\begin{array}{c}(\mathrm{R}-\mathrm{I})_{C} \\
(\mathrm{mag})\end{array}$ & $\begin{array}{c}T_{\text {eff }}{ }^{1} \\
(\mathrm{~K})\end{array}$ & $\begin{array}{c}T_{e f f}{ }^{2} \\
(\mathrm{~K})\end{array}$ & $\begin{array}{c}T_{\text {eff }} \pm 3 \sigma^{3} \\
(\mathrm{~K})\end{array}$ & $\begin{array}{l}\text { Spect. } \\
\text { Type }\end{array}$ & $\begin{array}{c}\pi \\
(\mathrm{m} ")\end{array}$ & $\begin{array}{c}M_{V} \\
(\mathrm{mag})\end{array}$ & $\begin{array}{c}R_{\star} \\
\left(R_{\odot}\right)\end{array}$ & {$[\mathrm{M} / \mathrm{H}]$} & $\begin{array}{l}\text { Hi. Res. } \\
\text { Spectra }\end{array}$ & Comments \\
\hline Gl 574 & 9.858 & 0.613 & 4474 & 4471 & $4472 \pm 1$ & $\mathrm{sdK} 4.5$ & $36.7356 \pm 0.0405$ & $7.683 \pm 0.022$ & $0.571 \pm 0.010$ & +0.17 & 14 & \\
\hline Gl 599.1 & 10.75 & 0.614 & 4471 & 4415 & $4443 \pm 28$ & sdK4.9 & $23.8051 \pm 0.0394$ & $7.633 \pm 0.024$ & $0.597 \pm 0.023$ & -1.00 & & \\
\hline Gl 604 & 8.055 & 0.560 & 4606 & 4587 & $4596 \pm 9$ & dK3.5 & $67.8795 \pm 0.0466$ & $7.214 \pm 0.021$ & $0.645 \pm 0.014$ & -0.28 & 41 & \\
\hline Gl 626 & 8.834 & 0.680 & 4306 & 4205 & $4255 \pm 50$ & dK6.1 & $57.0479 \pm 0.0414$ & $7.615 \pm 0.022$ & $0.694 \pm 0.040$ & -0.03 & & \\
\hline Gl 632.1 & 9.490 & 0.632 & 4426 & 4281 & $4353 \pm 72$ & dK5.5 & $37.2834 \pm 0.0243$ & $7.348 \pm 0.021$ & $0.729 \pm 0.054$ & & 3 & \\
\hline Gl 653 & 7.707 & 0.645 & 4394 & 4447 & $4420 \pm 27$ & dK5.1 & $95.5499 \pm 0.0651$ & $7.608 \pm 0.021$ & $0.615 \pm 0.023$ & -0.49 & 67 & \\
\hline Gl $659 \mathrm{~A}$ & 8.923 & 0.608 & 4486 & 4520 & $4503 \pm 17$ & dK4.1 & $47.0533 \pm 0.0269$ & $7.286 \pm 0.021$ & $0.670 \pm 0.019$ & +0.34 & & \\
\hline Gl 659B & 9.352 & 0.680 & 4306 & 4337 & $4321 \pm 16$ & dK5.7 & $47.0737 \pm 0.0244$ & $7.716 \pm 0.021$ & $0.630 \pm 0.018$ & +0.34 & & \\
\hline Gl 664 & 6.327 & 0.608 & 4486 & 4457 & $4471 \pm 14$ & dK4.5 & $168.0689 \pm 0.0758$ & $7.454 \pm 0.021$ & $0.635 \pm 0.017$ & -0.24 & 10 & \\
\hline Gl $677 \mathrm{~A}$ & 8.986 & 0.621 & 4454 & 4382 & $4418 \pm 36$ & dK5.1 & $42.40 \pm 3.76$ & $7.123 \pm 0.205$ & $0.770 \pm 0.105$ & -0.11 & & \\
\hline Gl 689 & 8.613 & 0.596 & 4516 & 4521 & $4518 \pm 3$ & dK3.9 & $52.4550 \pm 0.0261$ & $7.212 \pm 0.021$ & $0.686 \pm 0.012$ & -0.07 & 7 & \\
\hline Gl $690 \mathrm{~A}$ & 9.125 & 0.608 & 4486 & 4451 & $4468 \pm 17$ & dK4.6 & $41.3648 \pm 0.0234$ & $7.208 \pm 0.021$ & $0.713 \pm 0.020$ & -0.16 & 2 & \\
\hline Gl 698A & 9.186 & 0.656 & 4366 & 4397 & $4381 \pm 16$ & dK5.3 & $44.3596 \pm 0.0280$ & $7.421 \pm 0.021$ & $0.690 \pm 0.020$ & +0.20 & & \\
\hline Gl 702B & 6.00 & 0.620 & 4456 & 4475 & $4465 \pm 10$ & dK4.6 & $195.2166 \pm 0.1012$ & $7.453 \pm 0.021$ & $0.639 \pm 0.015$ & +0.09 & 4 & \\
\hline Gl 704B & 8.71 & 0.623 & 4449 & - & $4449 \pm 35$ & dK4.9 & $53.9769 \pm 0.6434$ & $7.371 \pm 0.046$ & $0.671 \pm 0.037$ & -0.54 & & \\
\hline Gl 707 & 8.372 & 0.675 & 4318 & 4338 & $4328 \pm 10$ & dK5.6 & $75.7454 \pm 0.0446$ & $7.769 \pm 0.021$ & $0.612 \pm 0.015$ & +0.34 & 13 & \\
\hline Gl 715 & 8.023 & 0.536 & 4711 & 4704 & $4707 \pm 3$ & $\mathrm{dK} 2.9$ & $53.2422 \pm 0.0700$ & $6.654 \pm 0.023$ & $0.767 \pm 0.013$ & +0.21 & 41 & \\
\hline Gl 717 & 9.995 & 0.67 & 4331 & 4321 & $4326 \pm 5$ & dK5.7 & $36.7812 \pm 0.0466$ & $7.823 \pm 0.023$ & $0.598 \pm 0.013$ & -0.29 & 42 & \\
\hline Gl $718 \mathrm{~A}$ & 8.883 & 0.584 & 4546 & 4554 & $4550 \pm 4$ & $\mathrm{dK} 3.8$ & $42.7481 \pm 0.0351$ & $7.038 \pm 0.022$ & $0.725 \pm 0.013$ & +0.16 & 13 & \\
\hline Gl $719 \mathrm{~A}$ & 9.01 & 0.600 & 4506 & 4424 & $4465 \pm 41$ & sdK4.6 & $60.5663 \pm 0.0366$ & $7.921 \pm 0.021$ & $0.515 \pm 0.024$ & +0.05 & & Subdwarf? \\
\hline Gl 719B & 9.01 & 0.600 & 4506 & 4424 & $4465 \pm 41$ & sdK4.6 & $60.5663 \pm 0.0366$ & $7.921 \pm 0.021$ & $0.515 \pm 0.024$ & +0.05 & & Subdwarf? \\
\hline Gl 727 & 7.912 & 0.560 & 4606 & 4591 & $4598 \pm 7$ & dK3.5 & $59.2621 \pm 0.0481$ & $6.776 \pm 0.022$ & $0.788 \pm 0.016$ & +0.12 & 2 & \\
\hline Gl $748.2 \mathrm{~A}$ & 10.199 & 0.657 & 4364 & 4462 & $4413 \pm 49$ & dK5.1 & $37.2248 \pm 0.0476$ & $8.053 \pm 0.023$ & $0.504 \pm 0.028$ & -0.13 & & Subdwarf? \\
\hline Gl $773.6 \mathrm{~A}$ & 9.058 & 0.554 & 4632 & 4410 & $4521 \pm 111$ & sdK3.9 & $47.8143 \pm 0.0463$ & $7.456 \pm 0.022$ & $0.611 \pm 0.066$ & -0.55 & 4 & \\
\hline Gl 773.6B & 9.058 & 0.554 & 4632 & 4410 & $4521 \pm 111$ & sdK3.9 & $47.8143 \pm 0.0463$ & $7.456 \pm 0.022$ & $0.611 \pm 0.066$ & -0.55 & 4 & \\
\hline Gl 775 & 7.457 & 0.584 & 4546 & 4591 & $4568 \pm 23$ & dK3.6 & $78.6238 \pm 0.0617$ & $6.935 \pm 0.022$ & $0.749 \pm 0.025$ & +0.11 & 37 & \\
\hline Gl 781.2 & 9.79 & 0.57 & 4581 & 4610 & $4595 \pm 15$ & dK3.5 & $31.8395 \pm 0.0467$ & $7.305 \pm 0.023$ & $0.619 \pm 0.017$ & -0.29 & 27 & \\
\hline Gl 782 & 8.901 & 0.687 & 4288 & 4378 & $4333 \pm 45$ & sdK5.6 & $63.2814 \pm 0.0714$ & $7.907 \pm 0.022$ & $0.572 \pm 0.030$ & -0.88 & 23 & \\
\hline Gl 787 & 8.708 & 0.585 & 4544 & 4461 & $4552 \pm 16$ & dK3.7 & $54.1449 \pm 0.0596$ & $7.376 \pm 0.022$ & $0.619 \pm 0.018$ & -0.29 & 31 & \\
\hline Gl 791.3 & 9.220 & 0.584 & 4546 & 4468 & $4507 \pm 39$ & $\mathrm{dK} 4.0$ & $37.1258 \pm 0.0330$ & $7.068 \pm 0.022$ & $0.739 \pm 0.035$ & & 3 & \\
\hline Gl $795 \mathrm{~A}$ & 8.634 & 0.694 & 4270 & 4300 & $4285 \pm 15$ & dK5.9 & $53.82 \pm 2.21$ & $7.289 \pm 0.107$ & $0.789 \pm 0.055$ & -0.23 & & Binary? \\
\hline Gl 795B & 8.634 & 0.694 & 4270 & 4300 & $4285 \pm 15$ & dK5.9 & $53.82 \pm 2.21$ & $7.289 \pm 0.107$ & $0.789 \pm 0.055$ & -0.23 & & Binary? \\
\hline Gl 818 & 8.272 & 0.680 & 4306 & 4316 & $4311 \pm 5$ & dK5.8 & $66.4656 \pm 0.0394$ & $7.385 \pm 0.021$ & $0.740 \pm 0.015$ & +0.12 & 1 & \\
\hline Gl $820 A$ & 5.205 & 0.644 & 4396 & 4362 & $4379 \pm 17$ & dK5.3 & $285.9459 \pm 0.1008$ & $7.486 \pm 0.021$ & $0.671 \pm 0.019$ & -0.23 & & \\
\hline Gl 836.3 & 8.833 & 0.565 & 4594 & 4561 & $4578 \pm 17$ & dK3.6 & $36.5805 \pm 0.3050$ & $6.649 \pm 0.038$ & $0.848 \pm 0.031$ & -0.10 & & Binary? \\
\hline Gl 845 & 5.75 & 0.56 & 4606 & 4684 & $4645 \pm 39$ & dK3.2 & $274.8048 \pm 0.2494$ & $7.945 \pm 0.022$ & $0.443 \pm 0.020$ & & & Subdwarf? \\
\hline Gl $848.1 \mathrm{~A}$ & 10.525 & 0.593 & 4524 & 4394 & $4459 \pm 65$ & dK4.7 & $21.7797 \pm 0.0432$ & $7.215 \pm 0.024$ & $0.716 \pm 0.049$ & +0.32 & & \\
\hline Gl 854 & 9.237 & 0.600 & 4506 & 4384 & $4445 \pm 61$ & dK4.9 & $41.6309 \pm 0.0229$ & $7.334 \pm 0.021$ & $0.685 \pm 0.043$ & +0.09 & 3 & \\
\hline Gl $857.1 \mathrm{~A}$ & 8.828 & 0.540 & 4693 & - & $4693 \pm 35$ & sdM2.9 & $47.7964 \pm 0.0631$ & $7.225 \pm 0.023$ & $0.596 \pm 0.025$ & & & Subdwarf? \\
\hline Gl 868 & 7.841 & 0.644 & 4396 & 4500 & $4448 \pm 52$ & dK4.8 & $75.2600 \pm 0.0511$ & $7.224 \pm 0.021$ & $0.719 \pm 0.041$ & +0.10 & & \\
\hline Gl 879 & 6.482 & 0.572 & 4576 & 4584 & $4580 \pm 4$ & dK3.6 & $131.4380 \pm 0.0856$ & $7.076 \pm 0.021$ & $0.696 \pm 0.013$ & -0.05 & 15 & \\
\hline Gl $889 \mathrm{~A}$ & 9.640 & 0.687 & 4288 & 4284 & $4286 \pm 2$ & dK5.9 & $45.2440 \pm 0.0528$ & $7.918 \pm 0.023$ & $0.590 \pm 0.011$ & & 5 & \\
\hline Gl 898 & 8.607 & 0.675 & 4318 & 4276 & $\begin{array}{l}4297 \pm 21 \\
\end{array}$ & dK5.8 & $68.6979 \pm 0.0581$ & $7.792 \pm 0.022$ & $0.620 \pm 0.021$ & +0.01 & 46 & \\
\hline HD 16909 & 8.210 & 0.566 & 4591 & 4401 & $4496 \pm 95$ & $\mathrm{dK} 4.2$ & $53.5579 \pm 0.1814$ & $6.854 \pm 0.027$ & $0.822 \pm 0.077$ & & & Binary? \\
\hline HD 18322 & 3.875 & 0.520 & 4780 & 4616 & $4698 \pm 82$ & K2.9III & $25.2039 \pm 0.3899$ & $0.882 \pm 0.053$ & $11.012 \pm 1.061$ & & 4 & \\
\hline HD 89668 & 9.40 & 0.578 & 4561 & 4641 & $4601 \pm 40$ & dK3.5 & $31.1705 \pm 0.0646$ & $6.869 \pm 0.024$ & $0.753 \pm 0.036$ & -0.03 & 6 & \\
\hline HD 90875 & 8.735 & 0.596 & 4516 & 4542 & $4529 \pm 13$ & dK3.9 & $42.4131 \pm 0.0403$ & $6.872 \pm 0.022$ & $0.795 \pm 0.021$ & +0.26 & & \\
\hline HD 96692 & 9.786 & 0.603 & 4499 & 4408 & $4454 \pm 46$ & dK4.7 & $35.4832 \pm 0.0496$ & $7.536 \pm 0.023$ & $0.620 \pm 0.032$ & -0.66 & & \\
\hline HD 107705B & 9.42 & 0.526 & 4754 & - & $4754 \pm 35$ & $\mathrm{dK} 2.8$ & $33.2810 \pm 0.0647$ & $7.031 \pm 0.024$ & $0.622 \pm 0.024$ & +0.07 & & \\
\hline HD 285507 & 10.49 & 0.585 & 4544 & - & $4544 \pm 35$ & dK3.8 & $22.1800 \pm 0.1188$ & $7.220 \pm 0.032$ & $0.670 \pm 0.032$ & & 5 & \\
\hline HD 286572 & 10.10 & 0.620 & 4456 & - & $4456 \pm 35$ & dK4.7 & $27.7687 \pm 0.0728$ & $7.318 \pm 0.026$ & $0.684 \pm 0.031$ & & 15 & \\
\hline HIP 948 & 9.95 & 0.614 & 4471 & - & $4471 \pm 35$ & dK4.5 & $28.6637 \pm 0.0475$ & $7.237 \pm 0.024$ & $0.702 \pm 0.031$ & & & \\
\hline HIP 24301 & 9.775 & 0.543 & 4680 & 4600 & $4640 \pm 40$ & sdK3.2 & $32.4351 \pm 0.0500$ & $7.330 \pm 0.023$ & $0.591 \pm 0.028$ & -0.84 & & \\
\hline
\end{tabular}


Table 2:: continued.

\begin{tabular}{|c|c|c|c|c|c|c|c|c|c|c|c|c|}
\hline Star & $\underset{(\mathrm{mag})}{\mathrm{V}}$ & $\begin{array}{c}(\mathrm{R}-\mathrm{I})_{C} \\
(\mathrm{mag})\end{array}$ & $\begin{array}{c}T_{e f f}^{1} \\
(\mathrm{~K})\end{array}$ & $\begin{array}{c}T_{e f f}^{2} \\
(\mathrm{~K})\end{array}$ & $\begin{array}{c}T_{e f f} \pm 3 \sigma^{3} \\
(\mathrm{~K})\end{array}$ & $\begin{array}{l}\text { Spect. } \\
\text { Type }\end{array}$ & $\begin{array}{c}\pi \\
(\mathrm{m} ")\end{array}$ & $\begin{array}{c}M_{V} \\
(\mathrm{mag})\end{array}$ & $\begin{array}{c}R_{\star} \\
\left(R_{\odot}\right)\end{array}$ & {$[\mathrm{M} / \mathrm{H}]$} & $\begin{array}{l}\text { Hi. Res. } \\
\text { Spectra }\end{array}$ & Comments \\
\hline HIP 28940 & 10.895 & 0.561 & 4603 & 4691 & $4647 \pm 44$ & sdK3.2 & $17.1457 \pm 0.0529$ & $7.066 \pm 0.027$ & $0.664 \pm 0.035$ & -0.68 & 3 & \\
\hline HIP 41277 & 10.365 & 0.609 & 4484 & - & $4484 \pm 35$ & $\mathrm{dK} 4.3$ & $23.7256 \pm 0.0607$ & $7.241 \pm 0.026$ & $0.694 \pm 0.031$ & & 2 & \\
\hline HIP 47333 & 10.495 & 0.651 & 4379 & - & $4379 \pm 35$ & sdK5.3 & $28.6394 \pm 0.0264$ & $7.780 \pm 0.022$ & $0.586 \pm 0.026$ & & & Subdwarf? \\
\hline HIP 91862 & 10.07 & 0.577 & 4564 & - & $4564 \pm 35$ & dK3.7 & $28.9071 \pm 0.0346$ & $7.375 \pm 0.023$ & $0.614 \pm 0.027$ & & & \\
\hline HIP 102582 & 9.73 & 0.576 & 4566 & - & $4566 \pm 35$ & dK3.7 & $31.6264 \pm 0.0508$ & $7.230 \pm 0.023$ & $0.655 \pm 0.029$ & & & \\
\hline HIP 106335 & 9.865 & 0.620 & 4456 & 4492 & $4474 \pm 18$ & $\mathrm{dK} 4.5$ & $23.2367 \pm 0.0412$ & $6.696 \pm 0.024$ & $0.899 \pm 0.027$ & -1.08 & 2 & Binary? \\
\hline LHS 1036 & 9.95 & 0.579 & 4559 & 4554 & $4557 \pm 3$ & dK3.7 & $28.6637 \pm 0.0475$ & $7.237 \pm 0.024$ & $0.658 \pm 0.012$ & -0.16 & 20 & \\
\hline LHS 1138 & 13.295 & 0.559 & 4610 & 4620 & $4615 \pm 5$ & sdK3.4 & $12.1535 \pm 0.0751$ & $8.719 \pm 0.033$ & $0.318 \pm 0.008$ & -2.49 & & \\
\hline LHS 1444 & 11.15 & 0.612 & 4476 & 4650 & $4563 \pm 87$ & sdK3.7 & $14.8825 \pm 0.0979$ & $7.013 \pm 0.034$ & $0.726 \pm 0.068$ & -1.08 & & Binary? \\
\hline LHS 1527 & 13.445 & 0.532 & 4728 & 4500 & $4614 \pm 114$ & sdK3.4 & $13.1245 \pm 0.0800$ & $9.035 \pm 0.033$ & $0.275 \pm 0.032$ & -1.59 & & \\
\hline LHS 1543 & 12.8 & 0.549 & 4654 & - & $4654 \pm 35$ & $\mathrm{sdK} 3.2$ & $13.1732 \pm 0.0605$ & $8.398 \pm 0.210$ & $0.357 \pm 0.049$ & -1.84 & 4 & \\
\hline LHS 1555 & 13.890 & 0.633 & 4424 & - & $4424 \pm 35$ & sdK5.1 & $12.7174 \pm 0.2106$ & $9.412 \pm 0.056$ & $0.267 \pm 0.016$ & -3.14 & & \\
\hline LHS 1776 & 10.825 & 0.549 & 4654 & 4458 & $4556 \pm 98$ & sdK3.7 & $22.4007 \pm 0.0394$ & $7.576 \pm 0.024$ & $0.563 \pm 0.055$ & -2.15 & & Binary? \\
\hline LHS 2161 & 11.570 & 0.584 & 4546 & 4500 & $4523 \pm 23$ & dK3.9 & $13.7826 \pm 0.0424$ & $7.267 \pm 0.027$ & $0.666 \pm 0.024$ & -0.32 & & \\
\hline LHS 2165 & 10.495 & 0.616 & 4466 & 4448 & $4457 \pm 9$ & sdK4.7 & $28.6394 \pm 0.0264$ & $7.780 \pm 0.022$ & $0.553 \pm 0.013$ & +0.02 & & \\
\hline LHS 2271 & 13.65 & 0.579 & 4559 & - & $4559 \pm 35$ & dK3.7 & $7.5744 \pm 0.0264$ & $8.047 \pm 0.028$ & $0.452 \pm 0.021$ & & 2 & Subdwarf? \\
\hline LHS 2321 & 9.840 & 0.561 & 4601 & 4517 & $4559 \pm 42$ & sdK3.7 & $23.5988 \pm 0.0446$ & $6.704 \pm 0.024$ & $0.839 \pm 0.042$ & -0.53 & & Binary? \\
\hline LHS 2463 & 12.49 & 0.532 & 4728 & 4540 & $4634 \pm 94$ & sdK3.3 & $12.9242 \pm 0.0399$ & $8.047 \pm 0.027$ & $0.427 \pm 0.040$ & -1.89 & & \\
\hline LHS 2558 & 10.72 & 0.627 & 4439 & 4500 & $4470 \pm 30$ & $\mathrm{sdK} 4.5$ & $25.4750 \pm 0.1046$ & $7.751 \pm 0.029$ & $0.555 \pm 0.023$ & -0.42 & & Subdwarf? \\
\hline LHS 2801 & 10.025 & 0.633 & 4424 & 4418 & $4421 \pm 3$ & dK5.1 & $31.9166 \pm 0.0459$ & $7.545 \pm 0.023$ & $0.632 \pm 0.012$ & +0.06 & & \\
\hline LHS 2869 & 11.058 & 0.609 & 4484 & - & $4484 \pm 35$ & $\mathrm{sdK} 4.3$ & $21.7339 \pm 0.0753$ & $7.744 \pm 0.028$ & $0.551 \pm 0.029$ & -0.45 & & Subdwarf? \\
\hline LHS 2938 & 10.725 & 0.605 & 4494 & 4490 & $4492 \pm 2$ & $\mathrm{dK} 4.2$ & $19.4753 \pm 0.0999$ & $7.172 \pm 0.031$ & $0.712 \pm 0.015$ & +0.14 & & \\
\hline LHS 2944 & 10.829 & 0.559 & 4610 & 4500 & $4555 \pm 55$ & dK3.7 & $19.0471 \pm 0.0352$ & $7.228 \pm 0.024$ & $0.661 \pm 0.040$ & -0.27 & & \\
\hline LHS 3074 & 11.35 & 0.607 & 4489 & - & $4489 \pm 35$ & $\mathrm{sdK} 4.3$ & $19.6547 \pm 0.0335$ & $7.817 \pm 0.024$ & $0.530 \pm 0.023$ & +0.31 & & Subdwarf? \\
\hline LHS 3110 & 12.330 & 0.639 & 4409 & 4600 & $4505 \pm 95$ & sdK4.0 & $11.9353 \pm 0.0351$ & $7.714 \pm 0.026$ & $0.550 \pm 0.053$ & -0.93 & & \\
\hline LHS $3172 \mathrm{~A}$ & 9.39 & 0.573 & 4573 & 4475 & $4524 \pm 49$ & dK3.9 & $37.1441 \pm 0.0551$ & $7.239 \pm 0.023$ & $0.674 \pm 0.038$ & -0.13 & & \\
\hline LHS 3172B & 9.70 & 0.573 & 4573 & 4475 & $4524 \pm 49$ & dK3.9 & $37.0752 \pm 0.0452$ & $7.545 \pm 0.023$ & $0.585 \pm 0.033$ & -0.13 & & \\
\hline LHS 3194 & 10.11 & 0.568 & 4586 & 4463 & $4525 \pm 61$ & dK3.9 & $20.5276 \pm 0.1939$ & $6.672 \pm 0.040$ & $0.875 \pm 0.065$ & +0.21 & & Binary? \\
\hline LHS 3266 & 13.61 & 0.628 & 4436 & - & $4436 \pm 35$ & sdK5.0 & $8.9056 \pm 0.0142$ & $8.358 \pm 0.023$ & $0.430 \pm 0.019$ & & & Subdwarf? \\
\hline LHS 3407 & 10.085 & 0.542 & 4684 & 4514 & $4599 \pm 85$ & $\mathrm{sdK} 3.5$ & $28.9071 \pm 0.0346$ & $7.390 \pm 0.023$ & $0.593 \pm 0.050$ & -0.25 & 1 & \\
\hline LHS 3426 & 10.63 & 0.547 & 4663 & 4644 & $4654 \pm 10$ & $\mathrm{dK} 3.2$ & $18.6398 \pm 0.0489$ & $6.982 \pm 0.026$ & $0.686 \pm 0.017$ & +0.34 & & \\
\hline LHS 3584 & 9.730 & 0.541 & 4689 & 4538 & $4614 \pm 75$ & $\mathrm{dK} 3.4$ & $31.6264 \pm 0.0508$ & $7.230 \pm 0.023$ & $0.631 \pm 0.048$ & -0.10 & & \\
\hline LHS 3742 & 10.48 & 0.531 & 4732 & 4619 & $4676 \pm 56$ & sdK3.0 & $20.9498 \pm 0.0407$ & $7.086 \pm 0.024$ & $0.643 \pm 0.039$ & -0.60 & & \\
\hline LHS 3986 & 11.616 & 0.600 & 4506 & 4285 & $4396 \pm 110$ & sdK5.2 & $12.0697 \pm 0.1213$ & $7.024 \pm 0.042$ & $0.819 \pm 0.094$ & -1.80 & & Binary? \\
\hline MCC 15 & 10.74 & 0.597 & 4514 & 4510 & $4512 \pm 2$ & $\mathrm{dK} 4.0$ & $21.6124 \pm 0.4269$ & $7.414 \pm 0.062$ & $0.628 \pm 0.023$ & -0.75 & & \\
\hline MCC 40 & 11.14 & 0.644 & 4396 & 4301 & $4348 \pm 47$ & dK5.5 & $17.8140 \pm 0.0429$ & $7.394 \pm 0.025$ & $0.716 \pm 0.040$ & +0.06 & & \\
\hline MCC 60 & 10.064 & 0.645 & 4394 & 4001 & $4394 \pm 35$ & sdK5.2 & $24.7518 \pm 0.3203$ & $7.032 \pm 0.048$ & $0.817 \pm 0.046$ & -0.86 & & Binary? \\
\hline MCC 108 & 10.61 & 0.585 & 4544 & 4500 & $4522 \pm 22$ & dK3.9 & $21.0030 \pm 0.0545$ & $7.221 \pm 0.026$ & $0.681 \pm 0.024$ & -0.08 & & \\
\hline MCC 147 & 10.27 & 0.549 & 4654 & 4623 & $4638 \pm 15$ & $\mathrm{dK} 3.2$ & $22.4300 \pm 0.0482$ & $7.024 \pm 0.025$ & $0.681 \pm 0.019$ & & 5 & \\
\hline MCC 151 & 10.48 & 0.668 & 4336 & 4349 & $4342 \pm 7$ & dK5.6 & $25.3088 \pm 0.0608$ & $7.496 \pm 0.025$ & $0.686 \pm 0.016$ & -0.16 & & \\
\hline MCC 175 & 10.645 & 0.645 & 4394 & 4344 & $4369 \pm 25$ & dK5.4 & $26.5797 \pm 0.0266$ & $7.768 \pm 0.022$ & $0.594 \pm 0.021$ & +0.33 & & \\
\hline MCC 240 & 9.145 & 0.573 & 4574 & 4410 & $4492 \pm 80$ & $\mathrm{dK} 4.2$ & $39.7680 \pm 0.0680$ & $7.143 \pm 0.024$ & $0.722 \pm 0.057$ & +0.04 & & \\
\hline MCC 241 & 10.145 & 0.526 & 4754 & 4724 & $4739 \pm 15$ & $\mathrm{dK} 2.8$ & $20.1559 \pm 0.4002$ & $6.667 \pm 0.063$ & $0.744 \pm 0.034$ & -0.44 & & \\
\hline MCC 248 & 10.880 & 0.549 & 4654 & 4513 & $4584 \pm 70$ & dK3.6 & $17.4788 \pm 0.0499$ & $7.093 \pm 0.026$ & $0.688 \pm 0.051$ & +0.00 & & \\
\hline MCC 249 & 10.232 & 0.573 & 4574 & 4528 & $4551 \pm 23$ & dK3.7 & $26.2703 \pm 0.0412$ & $7.329 \pm 0.023$ & $0.633 \pm 0.022$ & -0.17 & & \\
\hline MCC 251 & 10.052 & 0.562 & 4601 & - & $4601 \pm 35$ & dK3.5 & $27.7685 \pm 0.0440$ & $7.270 \pm 0.023$ & $0.626 \pm 0.027$ & -0.11 & & \\
\hline MCC 255 & 10.515 & 0.549 & 4654 & 4677 & $4666 \pm 11$ & dK3.1 & $20.9402 \pm 0.0379$ & $7.120 \pm 0.024$ & $0.638 \pm 0.015$ & -0.34 & & \\
\hline MCC 259 & 10.065 & 0.573 & 4574 & 4458 & $4516 \pm 58$ & dK3.9 & $27.4899 \pm 0.0539$ & $7.261 \pm 0.024$ & $0.671 \pm 0.043$ & -0.19 & & \\
\hline MCC 266 & 10.55 & 0.620 & 4456 & 4472 & $4464 \pm 16$ & dK4.6 & $22.9574 \pm 0.0429$ & $7.355 \pm 0.024$ & $0.669 \pm 0.020$ & & & \\
\hline MCC 274 & 10.356 & 0.633 & 4424 & 4500 & $4462 \pm 38$ & $\mathrm{dK} 4.6$ & $27.5571 \pm 0.0669$ & $7.557 \pm 0.025$ & $0.610 \pm 0.029$ & -0.04 & & \\
\hline MCC 276 & 9.82 & 0.585 & 4544 & 4517 & $4531 \pm 13$ & dK3.9 & $23.5988 \pm 0.0446$ & $6.684 \pm 0.024$ & $0.865 \pm 0.023$ & -0.53 & & Binary? \\
\hline MCC 287 & 10.920 & 0.633 & 4424 & 4451 & $4438 \pm 14$ & dK5.0 & $21.5030 \pm 0.0397$ & $7.582 \pm 0.024$ & $0.614 \pm 0.017$ & -0.24 & & \\
\hline
\end{tabular}


Table 2:: continued.

\begin{tabular}{|c|c|c|c|c|c|c|c|c|c|c|c|c|}
\hline Star & $\underset{(\mathrm{mag})}{\mathrm{V}}$ & $\begin{array}{c}(\mathrm{R}-\mathrm{I})_{C} \\
(\mathrm{mag})\end{array}$ & $\begin{array}{c}T_{e f f}^{1} \\
(\mathrm{~K})\end{array}$ & $\begin{array}{c}T_{e f f}^{2} \\
(\mathrm{~K})\end{array}$ & $\begin{array}{c}T_{e f f} \pm 3 \sigma^{3} \\
(\mathrm{~K})\end{array}$ & $\begin{array}{l}\text { Spect. } \\
\text { Type }\end{array}$ & $\begin{array}{c}\pi \\
(\mathrm{m} ")\end{array}$ & $\begin{array}{c}M_{V} \\
(\mathrm{mag})\end{array}$ & $\begin{array}{c}R_{\star} \\
\left(R_{\odot}\right)\end{array}$ & {$[\mathrm{M} / \mathrm{H}]$} & $\begin{array}{l}\text { Hi. Res. } \\
\text { Spectra }\end{array}$ & Comments \\
\hline MCC 317 & 10.18 & 0.584 & 4546 & 4573 & $4559 \pm 14$ & dK3.7 & $18.4988 \pm 0.0324$ & $6.516 \pm 0.024$ & $0.915 \pm 0.025$ & & & Binary? \\
\hline MCC 328 & 9.810 & 0.609 & 4484 & 4551 & $4518 \pm 33$ & dK3.9 & $34.5471 \pm 0.0403$ & $7.502 \pm 0.023$ & $0.600 \pm 0.026$ & +0.02 & & \\
\hline MCC 332A & 10.203 & 0.645 & 4394 & 4290 & $4342 \pm 52$ & sdK5.6 & $41.7694 \pm 0.9871$ & $8.307 \pm 0.071$ & $0.472 \pm 0.039$ & & 1 & Subdwarf? \\
\hline MCC 332B & 10.203 & 0.645 & 4394 & 4290 & $4342 \pm 52$ & sdK5.6 & $41.7694 \pm 0.9871$ & $8.307 \pm 0.071$ & $0.472 \pm 0.039$ & & 1 & Subdwarf? \\
\hline MCC 352A & 10.30 & 0.514 & 4806 & 4795 & $4801 \pm 6$ & dK??? & $11.4236 \pm 1.0559$ & $5.589 \pm 0.212$ & $1.173 \pm 0.130$ & -0.20 & & PMS? \\
\hline MCC $352 \mathrm{~B}$ & 10.74 & 0.537 & 4706 & 4736 & $4721 \pm 15$ & $\mathrm{dK} 2.9$ & $11.4236 \pm 1.0559$ & $6.029 \pm 0.212$ & $1.011 \pm 0.121$ & -0.20 & & PMS? \\
\hline MCC 359 & 11.00 & 0.573 & 4574 & 4443 & $4509 \pm 65$ & $\mathrm{dK} 4.0$ & $16.8438 \pm 0.0896$ & $7.132 \pm 0.032$ & $0.716 \pm 0.053$ & +0.50 & & \\
\hline MCC 363 & 9.10 & 0.537 & 4706 & 4636 & $4671 \pm 65$ & dK3.0 & $39.8944 \pm 0.0298$ & $7.105 \pm 0.022$ & $0.640 \pm 0.043$ & -0.14 & & \\
\hline MCC 373A & 10.85 & 0.526 & 4754 & 4672 & $4713 \pm 41$ & $\mathrm{dK} 2.8$ & $9.93 \pm 3.72$ & $5.835 \pm 0.711$ & $1.113 \pm 0.487$ & -0.31 & & PMS? \\
\hline MCC 373B & 10.85 & 0.526 & 4754 & 4672 & $4713 \pm 41$ & $\mathrm{dK} 2.8$ & $9.93 \pm 3.72$ & $5.835 \pm 0.711$ & $1.113 \pm 0.487$ & -0.31 & & PMS? \\
\hline MCC 376 & 10.638 & 0.609 & 4484 & 4416 & $4450 \pm 34$ & $\mathrm{dK} 4.8$ & $23.3065 \pm 0.0592$ & $7.475 \pm 0.026$ & $0.639 \pm 0.028$ & -1.64 & & Binary? \\
\hline MCC 377 & 10.82 & 0.633 & 4424 & 4484 & $4454 \pm 30$ & sdK4.7 & $24.3262 \pm 0.0321$ & $7.750 \pm 0.023$ & $0.561 \pm 0.022$ & -0.70 & & \\
\hline MCC 380 & 10.22 & 0.597 & 4514 & 4403 & $4459 \pm 56$ & $\mathrm{dK} 4.7$ & $25.7030 \pm 0.0597$ & $7.270 \pm 0.025$ & $0.698 \pm 0.043$ & +0.43 & & \\
\hline MCC 382 & 10.315 & 0.608 & 4486 & 4423 & $4454 \pm 31$ & $\mathrm{dK} 4.7$ & $23.7304 \pm 0.1408$ & $7.192 \pm 0.033$ & $0.726 \pm 0.033$ & +0.08 & & \\
\hline MCC 384 & 10.175 & 0.609 & 4484 & 4482 & $4483 \pm 1$ & $\mathrm{dK} 4.3$ & $25.5419 \pm 0.0513$ & $7.211 \pm 0.024$ & $0.704 \pm 0.012$ & +0.47 & & \\
\hline MCC 387 & 10.295 & 0.585 & 4544 & 4544 & $4544 \pm 1$ & dK3.8 & $26.8925 \pm 0.0657$ & $7.443 \pm 0.025$ & $0.604 \pm 0.011$ & -0.20 & & \\
\hline MCC 409 & 9.835 & 0.609 & 4484 & 4441 & $4462 \pm 21$ & $\mathrm{dK} 4.6$ & $32.3364 \pm 0.0438$ & $7.383 \pm 0.023$ & $0.661 \pm 0.021$ & +0.02 & 7 & \\
\hline MCC 441 & 9.810 & 0.597 & 4514 & 4580 & $4547 \pm 33$ & $\mathrm{dK} 3.8$ & $23.8993 \pm 0.1726$ & $6.702 \pm 0.036$ & $0.848 \pm 0.041$ & +0.29 & & Binary? \\
\hline MCC 448 & 10.69 & 0.668 & 4336 & 4378 & $4357 \pm 23$ & dK5.5 & $22.8329 \pm 0.0471$ & $7.483 \pm 0.024$ & $0.683 \pm 0.024$ & -0.10 & & \\
\hline MCC 475A & 9.840 & 0.633 & 4424 & - & $4424 \pm 35$ & dK5.1 & $33.4210 \pm 0.0593$ & $7.460 \pm 0.024$ & $0.656 \pm 0.029$ & +0.04 & & \\
\hline MCC 491 & 11.226 & 0.549 & 4654 & 4666 & $4660 \pm 6$ & $\mathrm{dK} 3.1$ & $14.4475 \pm 0.0585$ & $7.025 \pm 0.029$ & $0.670 \pm 0.015$ & -0.01 & & \\
\hline MCC 496 & 11.065 & 0.597 & 4514 & 4554 & $4534 \pm 20$ & $\mathrm{dK} 3.8$ & $17.5048 \pm 0.0486$ & $7.281 \pm 0.026$ & $0.656 \pm 0.022$ & -0.25 & & \\
\hline MCC 508 & 10.68 & 0.526 & 4754 & 4848 & $4801 \pm 47$ & dK??? & $12.5332 \pm 0.3682$ & $6.170 \pm 0.083$ & $0.897 \pm 0.067$ & +0.18 & & Binary? \\
\hline MCC 513 & 10.835 & 0.526 & 4754 & - & $4754 \pm 35$ & sdK2.6 & $25.5744 \pm 0.0406$ & $7.874 \pm 0.023$ & $0.422 \pm 0.016$ & -0.23 & & Subdwarf? \\
\hline MCC 515 & 10.826 & 0.645 & 4394 & 4389 & $4392 \pm 2$ & dK5.3 & $22.7375 \pm 0.0640$ & $7.610 \pm 0.026$ & $0.627 \pm 0.012$ & -0.36 & & \\
\hline MCC 519 & 10.692 & 0.549 & 4654 & 4732 & $4693 \pm 39$ & $\mathrm{dK} 2.9$ & $18.5970 \pm 0.0432$ & $7.039 \pm 0.025$ & $0.649 \pm 0.030$ & -0.20 & & \\
\hline MCC 522 & 10.365 & 0.644 & 4396 & 4391 & $4393 \pm 4$ & dK5.3 & $23.7256 \pm 0.0607$ & $7.241 \pm 0.026$ & $0.743 \pm 0.016$ & & & \\
\hline MCC 525 & 11.55 & 0.765 & 4092 & 4034 & $4063 \pm 29$ & $\mathrm{dK} 7.2$ & $15.7012 \pm 0.0426$ & $7.530 \pm 0.026$ & $0.856 \pm 0.035$ & -0.65 & & Binary? \\
\hline MCC 526 & 10.195 & 0.597 & 4513 & 4460 & $4486 \pm 26$ & $\mathrm{dK} 4.3$ & $28.3062 \pm 0.0442$ & $7.454 \pm 0.023$ & $0.628 \pm 0.023$ & & 15 & \\
\hline MCC 527 & 10.17 & 0.633 & 4424 & 4280 & $4352 \pm 72$ & dK5.5 & $28.1188 \pm 0.0662$ & $7.415 \pm 0.025$ & $0.707 \pm 0.054$ & & 1 & \\
\hline MCC 553 & 10.390 & 0.585 & 4544 & 4541 & $4543 \pm 2$ & dK3.8 & $18.2332 \pm 0.0456$ & $6.694 \pm 0.025$ & $0.853 \pm 0.016$ & -0.58 & & Binary? \\
\hline MCC 576 & 10.945 & 0.596 & 4516 & 4383 & $4449 \pm 66$ & $\mathrm{dK} 4.8$ & $16.9568 \pm 0.0442$ & $7.092 \pm 0.026$ & $0.763 \pm 0.053$ & -1.10 & & Binary? \\
\hline MCC 580 & 9.79 & 0.537 & 4706 & 4677 & $4692 \pm 14$ & $\operatorname{sdK} 2.9$ & $33.0816 \pm 0.2684$ & $7.388 \pm 0.038$ & $0.553 \pm 0.018$ & -0.93 & & \\
\hline MCC 590 & 10.08 & 0.537 & 4706 & 4692 & $4699 \pm 7$ & $\mathrm{dK} 2.8$ & $22.2720 \pm 0.0370$ & $6.819 \pm 0.024$ & $0.715 \pm 0.015$ & +0.11 & & \\
\hline MCC 592 & 10.077 & 0.537 & 4706 & 4705 & $4706 \pm 1$ & $\mathrm{dK} 2.8$ & $22.1425 \pm 0.0407$ & $6.803 \pm 0.024$ & $0.716 \pm 0.011$ & +0.01 & & \\
\hline MCC 595 & 10.005 & 0.514 & 4806 & 4699 & $4753 \pm 54$ & dK??? & $21.4515 \pm 0.0376$ & $6.662 \pm 0.024$ & $0.738 \pm 0.038$ & +0.06 & & \\
\hline MCC 620B & 11.78 & 0.561 & 4603 & 4385 & $4494 \pm 109$ & $\mathrm{dK} 4.2$ & $11.1954 \pm 0.1159$ & $7.025 \pm 0.042$ & $0.761 \pm 0.086$ & +0.68 & & \\
\hline MCC 628A & 10.065 & 0.561 & 4603 & 4663 & $4633 \pm 30$ & dK3.3 & $23.2051 \pm 0.0388$ & $6.893 \pm 0.024$ & $0.727 \pm 0.029$ & -0.21 & & \\
\hline MCC 628B & 10.065 & 0.561 & 4603 & 4663 & $4633 \pm 30$ & dK3.3 & $23.2051 \pm 0.0388$ & $6.893 \pm 0.024$ & $0.727 \pm 0.029$ & -0.21 & & \\
\hline MCC 629 & 11.19 & 0.632 & 4426 & - & $4426 \pm 35$ & dK5.1 & $16.3066 \pm 0.0430$ & $7.252 \pm 0.026$ & $0.721 \pm 0.033$ & & & \\
\hline
\end{tabular}


Table 2:: continued.

\begin{tabular}{|c|c|c|c|c|c|c|c|c|c|c|c|c|}
\hline Star & $\underset{(\mathrm{mag})}{\mathrm{V}}$ & $\begin{array}{c}(\mathrm{R}-\mathrm{I})_{C} \\
(\mathrm{mag})\end{array}$ & $\begin{array}{c}T_{e f f}^{1} \\
(\mathrm{~K})\end{array}$ & $\begin{array}{c}T_{e f f}^{2} \\
(\mathrm{~K})\end{array}$ & $\begin{array}{c}T_{e f f} \pm 3 \sigma^{3} \\
(\mathrm{~K})\end{array}$ & $\begin{array}{l}\text { Spect. } \\
\text { Type }\end{array}$ & $\begin{array}{c}\pi \\
(\mathrm{m} ")\end{array}$ & $\begin{array}{c}M_{V} \\
(\mathrm{mag})\end{array}$ & $\begin{array}{c}R_{\star} \\
\left(R_{\odot}\right)\end{array}$ & {$[\mathrm{M} / \mathrm{H}]$} & $\begin{array}{l}\text { Hi. Res. } \\
\text { Spectra }\end{array}$ & Comments \\
\hline MCC 631 & 10.575 & 0.584 & 4546 & 4446 & $4496 \pm 50$ & $\mathrm{dK} 4.2$ & $20.1504 \pm 0.0233$ & $7.096 \pm 0.023$ & $0.735 \pm 0.040$ & +0.07 & & \\
\hline MCC 643 & 10.445 & 0.573 & 4574 & 4514 & $4544 \pm 30$ & $\mathrm{dK} 3.8$ & $20.9927 \pm 0.0739$ & $7.055 \pm 0.028$ & $0.722 \pm 0.031$ & +0.06 & & \\
\hline MCC 657 & 10.45 & 0.597 & 4514 & 4447 & $4481 \pm 34$ & sdK4.3 & $26.9424 \pm 0.0234$ & $7.602 \pm 0.022$ & $0.589 \pm 0.025$ & -0.50 & & \\
\hline MCC 659 & 10.575 & 0.597 & 4514 & 4265 & $4390 \pm 124$ & dK5.3 & $23.2632 \pm 0.0264$ & $7.408 \pm 0.022$ & $0.689 \pm 0.081$ & +0.75 & & \\
\hline MCC 661 & 10.957 & 0.645 & 4394 & 4333 & $4364 \pm 30$ & dK5.4 & $20.6303 \pm 0.0544$ & $7.530 \pm 0.026$ & $0.665 \pm 0.028$ & +0.25 & & \\
\hline MCC 663 & 11.03 & 0.633 & 4424 & 4345 & $4384 \pm 39$ & dK5.3 & $18.2086 \pm 0.0406$ & $7.331 \pm 0.025$ & $0.718 \pm 0.035$ & -0.59 & & \\
\hline MCC 664 & 10.691 & 0.621 & 4454 & 4520 & $4487 \pm 33$ & $\mathrm{dK} 4.3$ & $23.0988 \pm 0.0400$ & $7.509 \pm 0.024$ & $0.612 \pm 0.026$ & -0.11 & & \\
\hline MCC 672 & 10.42 & 0.621 & 4454 & - & $4454 \pm 35$ & $\mathrm{dK} 4.7$ & $21.7506 \pm 0.1061$ & $7.107 \pm 0.031$ & $0.755 \pm 0.036$ & +0.17 & & \\
\hline MCC 678 & 11.28 & 0.549 & 4654 & 4862 & $4758 \pm 104$ & sdK2.6 & $18.6136 \pm 1.8408$ & $7.629 \pm 0.225$ & $0.471 \pm 0.090$ & -0.41 & & Subdwarf? \\
\hline MCC 683 & 10.260 & 0.645 & 4394 & 4419 & $4407 \pm 12$ & $\mathrm{dK} 5.2$ & $29.2771 \pm 0.0387$ & $7.593 \pm 0.023$ & $0.625 \pm 0.016$ & +0.07 & & \\
\hline MCC 698 & 10.005 & 0.633 & 4424 & 4394 & $4409 \pm 15$ & dK5.2 & $31.9166 \pm 0.0459$ & $7.525 \pm 0.023$ & $0.644 \pm 0.018$ & +0.06 & & \\
\hline MCC 703 & 10.270 & 0.621 & 4454 & 4457 & $4456 \pm 2$ & $\mathrm{dK} 4.7$ & $25.2560 \pm 0.0268$ & $7.282 \pm 0.022$ & $0.696 \pm 0.012$ & -0.08 & & \\
\hline MCC 710 & 10.040 & 0.621 & 4454 & 4416 & $4435 \pm 19$ & dK5.0 & $28.2407 \pm 0.0219$ & $7.294 \pm 0.022$ & $0.703 \pm 0.022$ & +0.35 & & \\
\hline MCC 712 & 10.86 & 0.561 & 4603 & 4619 & $4611 \pm 8$ & dK3.4 & $18.5318 \pm 0.0264$ & $7.200 \pm 0.023$ & $0.642 \pm 0.014$ & -0.71 & & \\
\hline MCC 717 & 9.91 & 0.621 & 4454 & 4414 & $4434 \pm 20$ & dK5.0 & $28.5387 \pm 0.0393$ & $7.187 \pm 0.023$ & $0.739 \pm 0.024$ & +0.29 & 6 & \\
\hline MCC $724 \mathrm{~A}$ & 10.97 & 0.585 & 4544 & 4524 & $4534 \pm 10$ & sdK3.8 & $13.1655 \pm 0.0319$ & $6.567 \pm 0.025$ & $0.911 \pm 0.023$ & -0.98 & & Binary? \\
\hline MCC 725 & 10.247 & 0.573 & 4574 & 4288 & $4431 \pm 143$ & dK5.0 & $23.5023 \pm 0.0436$ & $7.103 \pm 0.024$ & $0.770 \pm 0.103$ & +0.91 & & \\
\hline MCC 738 & 10.11 & 0.537 & 4706 & 4632 & $4669 \pm 37$ & dK3.1 & $20.4134 \pm 0.0429$ & $6.660 \pm 0.025$ & $0.787 \pm 0.036$ & +0.63 & & \\
\hline MCC $748 \mathrm{~B}$ & 11.11 & 0.621 & 4454 & 4536 & $4495 \pm 41$ & $\mathrm{dK} 4.2$ & $16.7063 \pm 0.0188$ & $7.224 \pm 0.022$ & $0.694 \pm 0.033$ & -0.37 & & \\
\hline MCC 754 & 10.827 & 0.621 & 4454 & 4343 & $4399 \pm 44$ & dK5.2 & $19.3023 \pm 0.3945$ & $7.255 \pm 0.064$ & $0.735 \pm 0.052$ & +0.18 & & \\
\hline MCC 757 & 10.375 & 0.621 & 4454 & 4257 & $4356 \pm 98$ & dK5.5 & $24.6896 \pm 0.0217$ & $7.338 \pm 0.022$ & $0.731 \pm 0.070$ & +0.08 & & \\
\hline MCC 768 & 10.079 & 0.645 & 4394 & 4105 & $4250 \pm 144$ & dK6.1 & $30.8429 \pm 0.0379$ & $7.525 \pm 0.023$ & $0.727 \pm 0.114$ & +0.38 & & \\
\hline MCC 773 & 10.397 & 0.609 & 4484 & 4554 & $4519 \pm 35$ & dK3.9 & $26.0960 \pm 0.0265$ & $7.480 \pm 0.022$ & $0.606 \pm 0.027$ & +0.05 & & \\
\hline MCC 778 & 9.60 & 0.596 & 4516 & 4628 & $4572 \pm 56$ & dK3.6 & $30.0928 \pm 0.0451$ & $6.992 \pm 0.023$ & $0.728 \pm 0.045$ & +0.03 & 8 & \\
\hline MCC 795A & 10.083 & 0.609 & 4484 & 4435 & $4460 \pm 24$ & sdK4.6 & $31.0905 \pm 0.0411$ & $7.546 \pm 0.023$ & $0.614 \pm 0.021$ & -0.70 & & Binary? \\
\hline MCC 795B & 10.083 & 0.609 & 4484 & 4435 & $4460 \pm 24$ & sdK4.6 & $31.0905 \pm 0.0411$ & $7.546 \pm 0.023$ & $0.614 \pm 0.021$ & -0.70 & & Binary? \\
\hline MCC 807 & 9.718 & 0.597 & 4514 & 4456 & $4485 \pm 29$ & $\mathrm{dK} 4.3$ & $29.1846 \pm 0.0265$ & $7.044 \pm 0.022$ & $0.760 \pm 0.029$ & -0.01 & & \\
\hline MCC 808 & 9.782 & 0.645 & 4394 & 4392 & $4393 \pm 1$ & dK5.3 & $35.0926 \pm 0.0546$ & $7.508 \pm 0.023$ & $0.657 \pm 0.012$ & +0.13 & & \\
\hline MCC 810 & 9.94 & 0.620 & 4456 & - & $4456 \pm 35$ & sdK4.7 & $34.4098 \pm 0.0386$ & $7.623 \pm 0.022$ & $0.594 \pm 0.026$ & & & \\
\hline MCC 822 & 10.12 & 0.609 & 4484 & 4495 & $4490 \pm 5$ & $\mathrm{dK} 4.2$ & $30.2977 \pm 0.0233$ & $7.527 \pm 0.022$ & $0.606 \pm 0.012$ & -0.08 & & \\
\hline MCC 829 & 9.87 & 0.584 & 4546 & 4478 & $4512 \pm 34$ & $\mathrm{dK} 4.0$ & $23.5105 \pm 0.0408$ & $6.726 \pm 0.024$ & $0.861 \pm 0.038$ & -0.07 & & Binary? \\
\hline MCC 847 & 10.185 & 0.526 & 4754 & 4765 & $4760 \pm 5$ & dK??? & $19.8272 \pm 0.0568$ & $6.671 \pm 0.026$ & $0.731 \pm 0.014$ & +0.31 & & \\
\hline MCC 849 & 10.672 & 0.645 & 4394 & - & $4394 \pm 35$ & dK5.3 & $24.9588 \pm 0.0876$ & $7.658 \pm 0.028$ & $0.613 \pm 0.028$ & -0.12 & & \\
\hline MCC 872 & 9.980 & 0.526 & 4754 & 4646 & $4700 \pm 54$ & dK2.9 & $23.7487 \pm 0.0462$ & $6.858 \pm 0.024$ & $0.702 \pm 0.041$ & -0.01 & & \\
\hline MCC 878 & 9.270 & 0.549 & 4654 & 4677 & $4666 \pm 11$ & dK3.1 & $35.5025 \pm 0.0506$ & $7.021 \pm 0.023$ & $0.668 \pm 0.016$ & +0.48 & & \\
\hline MCC 882 & 11.25 & 0.597 & 4514 & 4537 & $4526 \pm 11$ & sdK3.9 & $20.3831 \pm 0.0501$ & $7.796 \pm 0.025$ & $0.521 \pm 0.013$ & -0.32 & & Subdwarf? \\
\hline SAO 3300 & 10.325 & 0.600 & 4506 & - & $4506 \pm 35$ & $\mathrm{dK} 4.0$ & $22.05 \pm 1.70$ & $7.042 \pm 0.181$ & $0.748 \pm 0.092$ & & & \\
\hline STKM 1-83 & 10.315 & 0.549 & 4654 & 4550 & $4602 \pm 52$ & dK3.5 & $20.2828 \pm 0.0623$ & $6.851 \pm 0.027$ & $0.759 \pm 0.045$ & +0.09 & & \\
\hline STKM 1-98 & 9.48 & 0.526 & 4754 & 4750 & $4752 \pm 2$ & K2.5III & $2.1701 \pm 0.0682$ & $1.162 \pm 0.087$ & $9.293 \pm 0.430$ & +0.49 & & \\
\hline STKM 1-136 & 11.49 & 0.573 & 4574 & 4626 & $4600 \pm 26$ & dK 3.5 & $9.3979 \pm 0.0485$ & $6.355 \pm 0.031$ & $0.955 \pm 0.038$ & +0.21 & & Binary? \\
\hline STKM 1-158 & 9.815 & 0.549 & 4654 & - & $4654 \pm 35$ & dK3.1 & $26.7678 \pm 0.0777$ & $6.953 \pm 0.026$ & $0.696 \pm 0.031$ & +0.44 & & \\
\hline STKM 1-465 & 10.10 & 0.585 & 4544 & 4537 & $4540 \pm 4$ & dK 3.8 & $27.7687 \pm 0.0728$ & $7.318 \pm 0.026$ & $0.642 \pm 0.013$ & +0.34 & & \\
\hline STKM 1-475 & 11.195 & 0.597 & 4514 & 4541 & $4528 \pm 13$ & dK3.9 & $17.5486 \pm 0.0368$ & $7.416 \pm 0.025$ & $0.619 \pm 0.017$ & +0.15 & & \\
\hline STKM 1-480 & 10.820 & 0.621 & 4454 & 4477 & $4466 \pm 11$ & $\mathrm{dK} 4.6$ & $20.8956 \pm 0.0768$ & $7.420 \pm 0.028$ & $0.648 \pm 0.018$ & +0.02 & & \\
\hline STKM $1-489$ & 11.28 & 0.609 & 4484 & - & $4484 \pm 35$ & $\mathrm{dK} 4.3$ & $16.9481 \pm 0.0587$ & $7.426 \pm 0.028$ & $0.638 \pm 0.029$ & & 1 & \\
\hline STKM 1-518 & 10.61 & 0.561 & 4603 & 4522 & $4562 \pm 40$ & dK3.7 & $20.2409 \pm 0.0542$ & $7.141 \pm 0.026$ & $0.685 \pm 0.034$ & +0.39 & 1 & \\
\hline
\end{tabular}


Table 2:: continued.

\begin{tabular}{|c|c|c|c|c|c|c|c|c|c|c|c|c|}
\hline Star & $\underset{(\mathrm{mag})}{\mathrm{V}}$ & $\begin{array}{c}(\mathrm{R}-\mathrm{I})_{C} \\
(\mathrm{mag})\end{array}$ & $\begin{array}{c}T_{\text {eff }}{ }^{1} \\
(\mathrm{~K})\end{array}$ & $\begin{array}{c}T_{e f f}^{2} \\
(\mathrm{~K})\end{array}$ & $T_{\text {eff }} \pm 3 \sigma^{3}$ & $\begin{array}{l}\text { Spect. } \\
\text { Type }\end{array}$ & $\begin{array}{c}\pi \\
(\mathrm{m} ")\end{array}$ & $\begin{array}{c}M_{V} \\
(\mathrm{mag})\end{array}$ & $\begin{array}{c}R_{\star} \\
\left(R_{\odot}\right)\end{array}$ & {$[\mathrm{M} / \mathrm{H}]$} & $\begin{array}{l}\text { Hi. Res. } \\
\text { Spectra }\end{array}$ & Comments \\
\hline STKM 1-544 & 11.43 & 0.620 & 4456 & - & $4456 \pm 35$ & $\mathrm{dK} 4.7$ & $14.5389 \pm 0.0557$ & $7.243 \pm 0.028$ & $0.708 \pm 0.033$ & & & \\
\hline STKM 1-564 & 10.53 & 0.645 & 4394 & 4395 & $4395 \pm 1$ & dK5.2 & $24.7895 \pm 0.0442$ & $7.501 \pm 0.024$ & $0.658 \pm 0.012$ & -0.09 & & \\
\hline STKM 1-580 & 10.935 & 0.597 & 4514 & 4323 & $4419 \pm 95$ & dK5.1 & $19.3514 \pm 0.0295$ & $7.369 \pm 0.023$ & $0.687 \pm 0.064$ & +0.60 & & \\
\hline STKM 1-611 & 10.535 & 0.633 & 4424 & - & $4424 \pm 35$ & dK5.1 & $23.6491 \pm 0.0633$ & $7.404 \pm 0.026$ & $0.673 \pm 0.030$ & & & \\
\hline STKM 1-665 & 8.603 & 0.526 & 4754 & 4659 & $4707 \pm 47$ & dK2.8 & $45.7095 \pm 0.0451$ & $6.903 \pm 0.022$ & $0.684 \pm 0.035$ & -0.11 & & \\
\hline STKM 1-765 & 11.050 & 0.609 & 4484 & 4419 & $4452 \pm 32$ & $\mathrm{dK} 4.8$ & $19.0055 \pm 0.0261$ & $7.444 \pm 0.023$ & $0.647 \pm 0.027$ & +0.35 & & \\
\hline STKM 1-926 & 11.21 & 0.561 & 4603 & - & $4603 \pm 35$ & dK3.4 & $14.3656 \pm 0.0526$ & $6.997 \pm 0.028$ & $0.709 \pm 0.033$ & -0.23 & & \\
\hline STKM 1-964 & 11.63 & 0.585 & 4544 & - & $4544 \pm 35$ & dK3.8 & - & - & - & & & \\
\hline STKM 1-1118 & 10.190 & 0.561 & 4603 & - & $4603 \pm 35$ & dK3.4 & $24.8543 \pm 0.0369$ & $7.167 \pm 0.023$ & $0.655 \pm 0.029$ & -0.20 & & \\
\hline STKM 1-1227 & 10.43 & 0.585 & 4544 & 4347 & $4446 \pm 98$ & $\mathrm{dK} 4.9$ & $21.2210 \pm 0.0642$ & $7.064 \pm 0.027$ & $0.775 \pm 0.075$ & +0.46 & & \\
\hline STKM 1-1379 & 9.92 & 0.597 & 4514 & 4436 & $4475 \pm 39$ & $\mathrm{dK} 4.5$ & $29.9046 \pm 0.0284$ & $7.299 \pm 0.022$ & $0.681 \pm 0.031$ & +0.28 & & \\
\hline STKM 1-1489 & 11.15 & 0.549 & 4654 & 4492 & $4573 \pm 81$ & dK3.6 & $13.9195 \pm 0.0402$ & $6.868 \pm 0.026$ & $0.770 \pm 0.065$ & +0.34 & & \\
\hline STKM 1-1563 & 10.64 & 0.609 & 4484 & 4505 & $4495 \pm 10$ & $\mathrm{dK} 4.2$ & $19.9272 \pm 0.0194$ & $7.137 \pm 0.022$ & $0.722 \pm 0.017$ & -0.07 & & \\
\hline STKM 1-1767 & 10.305 & 0.645 & 4394 & 4351 & $4373 \pm 23$ & dK5.4 & $27.0517 \pm 0.0835$ & $7.466 \pm 0.027$ & $0.680 \pm 0.025$ & +0.36 & & \\
\hline STKM 1-1998 & 10.46 & 0.609 & 4484 & - & $4484 \pm 35$ & $\mathrm{dK} 4.3$ & $24.1672 \pm 0.0461$ & $7.376 \pm 0.024$ & $0.652 \pm 0.029$ & -0.08 & & \\
\hline G $127-107$ & 12.11 & 0.561 & 4603 & 4480 & $4542 \pm 61$ & $\operatorname{sdK} 3.8$ & $13.7920 \pm 0.0468$ & $7.808 \pm 0.027$ & $0.511 \pm 0.035$ & -1.52 & & \\
\hline G $145-38$ & 11.15 & 0.615 & 4469 & 4406 & $4438 \pm 33$ & $\operatorname{sdK5} .0$ & $17.6650 \pm 0.1690$ & $7.386 \pm 0.041$ & $0.672 \pm 0.034$ & -2.06 & & Binary? \\
\hline \multirow[t]{2}{*}{ PM 11088-1041 } & 9.230 & 0.627 & 4439 & 4413 & $4426 \pm 13$ & SdK5.1 & $50.3340 \pm 0.0478$ & $7.739 \pm 0.022$ & $0.576 \pm 0.015$ & -1.17 & & \\
\hline & & & & & Late-K sampl & & & & & & & \\
\hline GJ 1040 & 10.794 & 0.670 & 4331 & 4365 & $4348 \pm 17$ & sdK5.5 & $24.8206 \pm 0.1034$ & $7.768 \pm 0.029$ & $0.603 \pm 0.020$ & -0.84 & & \\
\hline GJ 1056 & 9.259 & 0.686 & 4290 & 4384 & $4337 \pm 47$ & dK5.6 & $45.5745 \pm 0.0318$ & $7.553 \pm 0.022$ & $0.671 \pm 0.036$ & & 8 & \\
\hline GJ 1066 & 8.847 & 0.680 & 4306 & 4365 & $4335 \pm 30$ & dK5.6 & $56.9195 \pm 0.0257$ & $7.623 \pm 0.021$ & $0.651 \pm 0.026$ & & 2 & \\
\hline GJ 1067 & 9.70 & 0.680 & 4306 & 4381 & $4343 \pm 38$ & dK5.6 & $38.6848 \pm 0.0305$ & $7.638 \pm 0.022$ & $0.643 \pm 0.030$ & & 2 & \\
\hline GJ 1107 & 10.14 & 0.719 & 4207 & 4250 & $4228 \pm 22$ & dK6.3 & $33.4765 \pm 0.0456$ & $7.764 \pm 0.023$ & $0.664 \pm 0.025$ & & & \\
\hline GJ 1127 & 9.406 & 0.700 & 4255 & 4305 & $4280 \pm 25$ & dK5.9 & $44.3476 \pm 0.0478$ & $7.640 \pm 0.022$ & $0.673 \pm 0.025$ & & & \\
\hline GJ 1150 & 9.534 & 0.676 & 4316 & 4296 & $4306 \pm 10$ & dK5.8 & $42.4308 \pm 0.0281$ & $7.672 \pm 0.021$ & $0.650 \pm 0.016$ & & & \\
\hline GJ $1177 \mathrm{~A}$ & 8.94 & 0.731 & 4177 & 4312 & $4244 \pm 68$ & PMS K6.2 & $68.4515 \pm 0.0640$ & $8.117 \pm 0.022$ & $0.556 \pm 0.045$ & +0.06 & 14 & \\
\hline GJ 1184 & 9.716 & 0.731 & 4177 & 4343 & $4260 \pm 83$ & dK6.1 & $33.0590 \pm 0.5902$ & $7.312 \pm 0.058$ & $0.795 \pm 0.082$ & +0.14 & & Binary? \\
\hline GJ 1213 & 10.18 & 0.781 & 4050 & 4281 & $4165 \pm 116$ & dK6.6 & $41.9536 \pm 0.0603$ & $8.294 \pm 0.023$ & $0.549 \pm 0.072$ & & & \\
\hline GJ 1257 & 11.098 & 0.688 & 4285 & 4285 & $4285 \pm 1$ & dK5.9 & $41.3937 \pm 0.0643$ & $9.183 \pm 0.023$ & $0.330 \pm 0.006$ & -0.23 & & Subdwarf? \\
\hline GJ 1267 & 8.992 & 0.800 & 4002 & 4050 & $4026 \pm 24$ & dK6.9 & $76.1461 \pm 0.0400$ & $8.400 \pm 0.021$ & $0.592 \pm 0.024$ & & 5 & \\
\hline GJ 1279 & 8.39 & 0.686 & 4290 & 4395 & $4342 \pm 53$ & dK5.6 & $67.3960 \pm 0.0382$ & $7.533 \pm 0.021$ & $0.675 \pm 0.039$ & -0.10 & 1 & \\
\hline GJ 3048 & 10.97 & 0.793 & 4020 & - & $4020 \pm 41$ & dK7.4 & $22.1804 \pm 0.0434$ & $7.700 \pm 0.024$ & $0.822 \pm 0.049$ & & & Binary? \\
\hline GJ 3072 & 9.993 & 0.778 & 4058 & 4053 & $4055 \pm 3$ & $\mathrm{dK} 7.2$ & $47.3703 \pm 0.0397$ & $8.371 \pm 0.022$ & $0.585 \pm 0.013$ & & 1 & \\
\hline GJ 3199 & 9.650 & 0.719 & 4207 & 4148 & $4178 \pm 30$ & dK6.6 & $37.0209 \pm 0.2243$ & $7.492 \pm 0.033$ & $0.786 \pm 0.040$ & -0.21 & & Binary? \\
\hline GJ 3269 & 10.97 & 0.657 & 4364 & 4325 & $4344 \pm 19$ & dK5.6 & $21.9414 \pm 0.0690$ & $7.676 \pm 0.027$ & $0.631 \pm 0.021$ & & 1 & \\
\hline GJ 3411 & 10.16 & 0.731 & 4177 & 4291 & $4234 \pm 57$ & dK6.2 & $36.3127 \pm 0.0388$ & $7.960 \pm 0.022$ & $0.603 \pm 0.042$ & +0.48 & 3 & \\
\hline GJ 3433 & 9.945 & 0.732 & 4174 & 4250 & $4212 \pm 38$ & dK6.4 & $42.8025 \pm 0.0480$ & $8.102 \pm 0.022$ & $0.576 \pm 0.030$ & & & \\
\hline GJ 3494 & 9.748 & 0.740 & 4154 & 4261 & $4207 \pm 54$ & dK6.4 & $44.0229 \pm 0.0437$ & $7.966 \pm 0.022$ & $0.616 \pm 0.042$ & & & \\
\hline GJ 3546 & 9.54 & 0.719 & 4207 & 4343 & $4275 \pm 32$ & dK6.0 & $47.6539 \pm 0.0507$ & $7.930 \pm 0.022$ & $0.591 \pm 0.025$ & +0.19 & & \\
\hline GJ 3551 & 9.531 & 0.728 & 4184 & 4306 & $4245 \pm 61$ & $\mathrm{dK} 6.2$ & $42.7802 \pm 0.0263$ & $7.687 \pm 0.021$ & $0.677 \pm 0.050$ & -0.40 & & \\
\hline GJ 3608 & 10.19 & 0.756 & 4114 & 4060 & $4087 \pm 27$ & $\mathrm{dK} 7.1$ & $29.4215 \pm 0.0625$ & $7.533 \pm 0.025$ & $0.836 \pm 0.038$ & -0.32 & 1 & Binary? \\
\hline GJ 3674 & 9.970 & 0.768 & 4083 & 4209 & $4146 \pm 63$ & dK6.8 & $41.2662 \pm 0.0484$ & $8.048 \pm 0.023$ & $0.626 \pm 0.049$ & & & \\
\hline GJ 3883 & 9.988 & 0.781 & 4050 & 4106 & $4078 \pm 28$ & dK7.1 & $38.8276 \pm 0.0291$ & $7.934 \pm 0.022$ & $0.701 \pm 0.031$ & & & \\
\hline GJ 3906 & 9.87 & 0.706 & 4240 & 4224 & $4232 \pm 8$ & $\mathrm{dK} 6.2$ & $36.5938 \pm 0.0498$ & $7.687 \pm 0.023$ & $0.685 \pm 0.017$ & +0.72 & & \\
\hline GJ 3957 & 9.62 & 0.645 & 4394 & 4274 & $4334 \pm 60$ & dK5.6 & $35.8254 \pm 0.1712$ & $7.391 \pm 0.030$ & $0.725 \pm 0.050$ & & & \\
\hline GJ 3996 & 10.333 & 0.812 & 3985 & 4046 & $4015 \pm 31$ & dK7.4 & $38.3099 \pm 0.0493$ & $8.250 \pm 0.023$ & $0.641 \pm 0.031$ & -0.11 & & \\
\hline GJ 4140 & 10.168 & 0.812 & 3985 & 4030 & $4007 \pm 23$ & $\mathrm{dK} 7.4$ & $46.1956 \pm 0.0439$ & $8.491 \pm 0.022$ & $0.578 \pm 0.024$ & +0.42 & 1 & \\
\hline GJ 9073B & 10.49 & 0.719 & 4207 & - & $4207 \pm 41$ & dK6.4 & $26.9229 \pm 0.0458$ & $7.641 \pm 0.024$ & $0.715 \pm 0.040$ & +0.09 & & \\
\hline
\end{tabular}


Table 2:: continued.

\begin{tabular}{|c|c|c|c|c|c|c|c|c|c|c|c|c|}
\hline Star & $\begin{array}{c}\mathrm{V} \\
(\mathrm{mag})\end{array}$ & $\begin{array}{c}(\mathrm{R}-\mathrm{I})_{C} \\
(\mathrm{mag})\end{array}$ & $\begin{array}{c}T_{\text {eff }}{ }^{1} \\
(\mathrm{~K})\end{array}$ & $\begin{array}{c}T_{e f f}{ }^{2} \\
(\mathrm{~K})\end{array}$ & $T_{\text {eff }} \pm 3 \sigma^{3}$ & $\begin{array}{l}\text { Spect. } \\
\text { Type }\end{array}$ & $\begin{array}{c}\pi \\
(\mathrm{m} ")\end{array}$ & $\begin{array}{c}M_{V} \\
(\mathrm{mag})\end{array}$ & $\begin{array}{c}R_{\star} \\
\left(R_{\odot}\right)\end{array}$ & {$[\mathrm{M} / \mathrm{H}]$} & $\begin{array}{l}\text { Hi. Res. } \\
\text { Spectra }\end{array}$ & Comments \\
\hline GJ 9299 & 9.742 & 0.728 & 4184 & 4368 & $4276 \pm 92$ & dK6.0 & $41.3591 \pm 0.0590$ & $7.825 \pm 0.023$ & $0.620 \pm 0.057$ & +0.15 & & \\
\hline GJ 9323A & 10.176 & 0.682 & 4300 & 4326 & $4313 \pm 13$ & dK5.7 & $36.3587 \pm 0.0556$ & $7.979 \pm 0.023$ & $0.562 \pm 0.016$ & +0.30 & & \\
\hline GJ 9354 & 10.536 & 0.694 & 4270 & 4332 & $4301 \pm 31$ & dK5.8 & $28.3367 \pm 0.0625$ & $7.798 \pm 0.025$ & $0.616 \pm 0.026$ & +0.33 & & \\
\hline GJ 9358 & 10.375 & 0.768 & 4083 & - & $4083 \pm 41$ & $\mathrm{dK} 7.1$ & $31.2347 \pm 0.8082$ & $7.848 \pm 0.075$ & $0.726 \pm 0.061$ & & 4 & \\
\hline GJ 9372 & 9.585 & 0.676 & 4316 & 4460 & $4388 \pm 28$ & dK5.3 & $32.2570 \pm 0.2491$ & $7.128 \pm 0.037$ & $0.786 \pm 0.036$ & -0.20 & & Binary? \\
\hline GJ 9412 & 10.51 & 0.768 & 4083 & 4073 & $4078 \pm 5$ & $\mathrm{dK} 7.1$ & $24.4343 \pm 0.1955$ & $7.450 \pm 0.037$ & $0.876 \pm 0.027$ & & & Binary? \\
\hline GJ 9452 & 10.226 & 0.700 & 4255 & 4368 & $4312 \pm 56$ & sdK5.7 & $28.1847 \pm 0.0206$ & $7.476 \pm 0.022$ & $0.709 \pm 0.043$ & -0.59 & & \\
\hline Gl 9464 & 10.317 & 0.706 & 4240 & & $4240 \pm 41$ & dK6.2 & $28.3819 \pm 0.0364$ & $7.582 \pm 0.023$ & $0.714 \pm 0.040$ & & & \\
\hline GJ $9490 \mathrm{C}$ & 10.057 & 0.768 & 4083 & 4170 & $4127 \pm 43$ & dK6.9 & $42.2410 \pm 0.0469$ & $8.186 \pm 0.022$ & $0.597 \pm 0.035$ & +0.20 & & \\
\hline GJ $9490 \mathrm{~A}$ & 9.043 & 0.719 & 4207 & 4300 & $4254 \pm 47$ & $\mathrm{dK} 6.1$ & $42.2410 \pm 0.0469$ & $7.172 \pm 0.022$ & $0.852 \pm 0.047$ & +0.20 & & Binary? \\
\hline GJ 9490B & 10.035 & 0.719 & 4207 & 4300 & $4254 \pm 47$ & dK6.1 & $42.2410 \pm 0.0469$ & $8.164 \pm 0.022$ & $0.540 \pm 0.030$ & +0.20 & & \\
\hline GJ 9506 & 10.513 & 0.768 & 4083 & - & $4083 \pm 41$ & $\mathrm{dK} 7.1$ & $27.0340 \pm 0.1664$ & $7.673 \pm 0.033$ & $0.787 \pm 0.049$ & -0.13 & & Binary? \\
\hline GJ 9518 & 10.34 & 0.700 & 4255 & - & $4255 \pm 41$ & dK6.1 & $36.3923 \pm 0.0805$ & $8.145 \pm 0.025$ & $0.544 \pm 0.028$ & & & Subdwarfs? \\
\hline GJ $9522 \mathrm{~A}$ & 10.389 & 0.694 & 4270 & - & $4270 \pm 41$ & dK6.0 & $30.7613 \pm 0.0212$ & $7.829 \pm 0.021$ & $0.622 \pm 0.031$ & & & \\
\hline GJ 9667 & 9.958 & 0.698 & 4260 & 4374 & $4317 \pm 57$ & dK5.7 & $38.3851 \pm 0.0419$ & $7.879 \pm 0.022$ & $0.587 \pm 0.037$ & +0.09 & 1 & \\
\hline GJ 9684 & 10.855 & 0.721 & 4202 & 4308 & $4255 \pm 53$ & dK6.1 & $25.9378 \pm 0.0404$ & $7.925 \pm 0.023$ & $0.602 \pm 0.036$ & -0.04 & & \\
\hline GJ 9714 & 9.326 & 0.728 & 4184 & 4344 & $4264 \pm 80$ & dK6.0 & $48.7229 \pm 0.0582$ & $7.765 \pm 0.023$ & $0.644 \pm 0.053$ & +0.10 & & \\
\hline GJ $9721 \mathrm{~A}$ & 9.44 & 0.700 & 4255 & 4290 & $4272 \pm 18$ & dK6.0 & $41.7694 \pm 0.9871$ & $7.544 \pm 0.071$ & $0.708 \pm 0.039$ & & 1 & \\
\hline GJ 9805 & 11.452 & 0.756 & 4114 & 4086 & $4100 \pm 14$ & $\mathrm{dK} 7.0$ & $21.9889 \pm 0.0606$ & $8.163 \pm 0.026$ & $0.618 \pm 0.021$ & +0.45 & & \\
\hline GJ 9827 & 10.39 & 0.716 & 4215 & 4250 & $4233 \pm 17$ & dK6.3 & $32.6855 \pm 0.0611$ & $7.962 \pm 0.024$ & $0.603 \pm 0.020$ & & 9 & \\
\hline GJ 9846 & 10.718 & 0.694 & 4270 & 4286 & $4278 \pm 8$ & dK6.0 & $22.7434 \pm 0.0613$ & $7.502 \pm 0.026$ & $0.719 \pm 0.018$ & -0.67 & & \\
\hline Gl 14 & 9.00 & 0.812 & 3985 & 4020 & $4002 \pm 18$ & $\mathrm{dK} 7.4$ & $68.0351 \pm 0.0566$ & $8.164 \pm 0.022$ & $0.675 \pm 0.024$ & -0.05 & 2 & \\
\hline Gl 31.2 & 10.722 & 0.805 & 3995 & 3970 & $3983 \pm 13$ & $\mathrm{dK} 7.5$ & $27.4141 \pm 0.5302$ & $7.912 \pm 0.062$ & $0.774 \pm 0.041$ & +0.32 & & Binary? \\
\hline Gl $40 \mathrm{~A}$ & 8.930 & 0.702 & 4250 & 4253 & $4251 \pm 2$ & sdK6.1 & $65.1905 \pm 0.0567$ & $8.001 \pm 0.022$ & $0.583 \pm 0.011$ & -0.61 & 2 & \\
\hline Gl 45 & 9.482 & 0.696 & 4265 & 4206 & $4235 \pm 29$ & $\mathrm{dK} 6.2$ & $47.7655 \pm 0.0244$ & $7.878 \pm 0.021$ & $0.626 \pm 0.027$ & +0.00 & 4 & \\
\hline Gl 50 & 10.47 & 0.684 & 4295 & 4344 & $4319 \pm 25$ & dK5.7 & $29.0239 \pm 0.0353$ & $7.784 \pm 0.023$ & $0.612 \pm 0.022$ & +0.56 & & \\
\hline Gl 52 & 8.97 & 0.740 & 4154 & 4123 & $4138 \pm 15$ & dK6.8 & $66.3671 \pm 0.0375$ & $8.080 \pm 0.021$ & $0.621 \pm 0.020$ & & 3 & \\
\hline Gl 53.2 & 10.519 & 0.723 & 4197 & 4250 & $4224 \pm 26$ & dK6.3 & $34.9671 \pm 0.0578$ & $8.237 \pm 0.024$ & $0.535 \pm 0.022$ & & & Subdwarfs? \\
\hline Gl 57 & 10.130 & 0.874 & 3902 & 3906 & $3904 \pm 2$ & dK7.9 & $59.8670 \pm 0.0262$ & $9.016 \pm 0.021$ & $0.508 \pm 0.011$ & & 3 & \\
\hline GL 105.5 & 9.509 & 0.680 & 4306 & 4283 & $4294 \pm 11$ & dK5.9 & $41.7670 \pm 0.0403$ & $7.613 \pm 0.022$ & $0.675 \pm 0.017$ & -0.01 & 1 & \\
\hline Gl 106.1C & 10.127 & 0.731 & 4177 & 4193 & $4185 \pm 8$ & dK6.5 & $40.6395 \pm 0.0479$ & $8.172 \pm 0.023$ & $0.571 \pm 0.014$ & +1.03 & & \\
\hline Gl 112.1 & 8.574 & 0.664 & 4346 & 4373 & $4359 \pm 14$ & dK5.5 & $61.9128 \pm 0.0461$ & $7.533 \pm 0.022$ & $0.666 \pm 0.018$ & & & \\
\hline GL 116 & 9.608 & 0.725 & 4192 & 4290 & $4241 \pm 49$ & sdK6.2 & $67.7365 \pm 0.0380$ & $8.762 \pm 0.021$ & $0.414 \pm 0.026$ & & 6 & Subdwarfs? \\
\hline Gl 123 & 9.055 & 0.783 & 4045 & 4110 & $4077 \pm 33$ & $\mathrm{dK} 7.1$ & $67.8892 \pm 0.0518$ & $8.214 \pm 0.022$ & $0.617 \pm 0.030$ & & & \\
\hline Gl 142 & 8.367 & 0.752 & 4124 & 4172 & $4148 \pm 24$ & dK6.7 & $80.04 \pm 0.99$ & $7.884 \pm 0.047$ & $0.674 \pm 0.035$ & -0.13 & 1 & \\
\hline Gl 143.1 & 9.991 & 0.799 & 4005 & 4073 & $4039 \pm 34$ & dK 7.3 & $47.5363 \pm 0.0348$ & $8.376 \pm 0.022$ & $0.592 \pm 0.030$ & +0.45 & 4 & \\
\hline Gl 146 & 8.60 & 0.725 & 4192 & 4252 & $4222 \pm 30$ & $\mathrm{dK} 6.3$ & $73.5244 \pm 0.0387$ & $7.932 \pm 0.021$ & $0.617 \pm 0.027$ & +0.38 & 3 & \\
\hline Gl 148A & 10.392 & 0.779 & 4056 & 4152 & $4104 \pm 48$ & $\mathrm{dK} 7.0$ & $38.7108 \pm 0.0647$ & $8.331 \pm 0.024$ & $0.570 \pm 0.037$ & +0.77 & & \\
\hline Gl 148B & 10.392 & 0.779 & 4056 & 4152 & $4104 \pm 48$ & dK7.0 & $38.7108 \pm 0.0647$ & $8.331 \pm 0.024$ & $0.570 \pm 0.037$ & +0.77 & & \\
\hline Gl 153A & 9.35 & 0.728 & 4184 & 4190 & $4187 \pm 3$ & dK6.5 & $55.8339 \pm 0.0343$ & $8.084 \pm 0.021$ & $0.593 \pm 0.012$ & +0.04 & 2 & \\
\hline Gl 156 & 9.015 & 0.750 & 4129 & 4006 & $4067 \pm 61$ & $\mathrm{dK} 7.2$ & $63.7059 \pm 0.0468$ & $8.036 \pm 0.022$ & $0.675 \pm 0.052$ & & 1 & \\
\hline Gl 182 & 10.107 & 1.37 & 4073 & - & $4073 \pm 41$ & PMS K7.1 & $40.9812 \pm 0.0338$ & $8.170 \pm 0.022$ & $0.631 \pm 0.036$ & & 6 & \\
\hline Gl $186 \mathrm{~A}$ & 10.02 & 0.744 & 4144 & 4272 & $4208 \pm 64$ & dK6.4 & $43.0961 \pm 0.3615$ & $8.192 \pm 0.038$ & $0.554 \pm 0.047$ & +0.09 & & \\
\hline Gl 186B & 10.02 & 0.744 & 4144 & 4272 & $4208 \pm 64$ & dK6.4 & $43.0961 \pm 0.3615$ & $8.192 \pm 0.038$ & $0.554 \pm 0.047$ & +0.09 & & \\
\hline Gl 208 & 8.841 & 0.801 & 4000 & 3975 & $3987 \pm 24$ & $\mathrm{dK} 7.5(\mathrm{e})$ & $87.4367 \pm 0.0562$ & $8.549 \pm 0.021$ & $0.574 \pm 0.027$ & +0.01 & 52 & \\
\hline Gl 221 & 9.693 & 0.744 & 4144 & 4122 & $4133 \pm 11$ & dK6.8 & $49.3062 \pm 0.0529$ & $8.158 \pm 0.022$ & $0.602 \pm 0.017$ & +0.19 & 44 & \\
\hline Gl 226.2 & 9.75 & 0.645 & 4394 & 4393 & $4393 \pm 1$ & dK5.3 & $39.9945 \pm 0.0290$ & $7.760 \pm 0.022$ & $0.585 \pm 0.010$ & & & Subdwarfs? \\
\hline Gl 249 & 8.96 & 0.657 & 4364 & 4348 & $4356 \pm 16$ & dK5.5 & $53.5873 \pm 0.0377$ & $7.605 \pm 0.022$ & $0.646 \pm 0.019$ & -0.17 & 20 & \\
\hline Gl $265 \mathrm{~A}$ & 10.184 & 0.743 & 4146 & 4139 & $4143 \pm 4$ & $\mathrm{dK} 6.8$ & $43.7567 \pm 0.0513$ & $8.389 \pm 0.023$ & $0.536 \pm 0.012$ & & & \\
\hline Gl 266 & 9.87 & 0.682 & 4301 & 4203 & $4252 \pm 49$ & dK6.1 & $38.2618 \pm 0.2672$ & $7.784 \pm 0.035$ & $0.644 \pm 0.041$ & +0.17 & 10 & \\
\hline Gl 287 & 10.170 & 0.758 & 4109 & 4130 & $4119 \pm 11$ & dK6.9 & $37.5791 \pm 0.0543$ & $8.045 \pm 0.023$ & $0.642 \pm 0.019$ & +0.20 & 2 & \\
\hline Gl 296 & 9.65 & 0.765 & 4091 & 4037 & $4064 \pm 27$ & dK 7.2 & $47.2484 \pm 0.0293$ & $8.022 \pm 0.021$ & $0.681 \pm 0.030$ & & & \\
\hline
\end{tabular}


Table 2:: continued.

\begin{tabular}{|c|c|c|c|c|c|c|c|c|c|c|c|c|}
\hline Star & $\begin{array}{c}\mathrm{V} \\
(\mathrm{mag})\end{array}$ & $\begin{array}{c}(\mathrm{R}-\mathrm{I})_{C} \\
(\mathrm{mag})\end{array}$ & $\begin{array}{c}T_{\text {eff }}{ }^{1} \\
(\mathrm{~K})\end{array}$ & $\begin{array}{c}T_{e f f}{ }^{2} \\
(\mathrm{~K})\end{array}$ & $\begin{array}{c}T_{\text {eff }} \pm 3 \sigma^{3} \\
(\mathrm{~K})\end{array}$ & $\begin{array}{l}\text { Spect. } \\
\text { Type }\end{array}$ & $\begin{array}{c}\pi \\
(\mathrm{m} ")\end{array}$ & $\begin{array}{c}M_{V} \\
(\mathrm{mag})\end{array}$ & $\begin{array}{c}R_{\star} \\
\left(R_{\odot}\right)\end{array}$ & {$[\mathrm{M} / \mathrm{H}]$} & $\begin{array}{l}\text { Hi. Res. } \\
\text { Spectra }\end{array}$ & Comments \\
\hline Gl 322 & 9.28 & 0.764 & 4093 & 4237 & $4165 \pm 72$ & dK6.6 & $60.5523 \pm 0.0303$ & $8.191 \pm 0.021$ & $0.576 \pm 0.049$ & & 3 & \\
\hline Gl 343.1 & 9.845 & 0.706 & 4240 & - & $4240 \pm 41$ & dK6.2 & $30.6014 \pm 0.0681$ & $7.274 \pm 0.025$ & $0.823 \pm 0.046$ & & & Binary? \\
\hline Gl $350 \mathrm{~A}$ & 11.001 & 0.719 & 4207 & - & $4207 \pm 41$ & $\mathrm{dK} 6.4$ & $26.3248 \pm 0.0595$ & $8.103 \pm 0.025$ & $0.578 \pm 0.033$ & -0.03 & & \\
\hline Gl 369 & 10.016 & 0.780 & 4053 & 3932 & $3992 \pm 60$ & $\mathrm{sdK} 7.5$ & $76.2323 \pm 0.0475$ & $9.427 \pm 0.021$ & $0.381 \pm 0.034$ & -0.20 & 51 & Subdwarfs? \\
\hline Gl 379A & 10.241 & 0.756 & 4114 & 4290 & $4202 \pm 88$ & dK6.4 & $47.4964 \pm 0.0432$ & $8.624 \pm 0.022$ & $0.457 \pm 0.046$ & & & Subdwarfs? \\
\hline Gl $379 \mathrm{~B}$ & 10.267 & 0.756 & 4114 & 4290 & $4202 \pm 88$ & $\mathrm{dK} 6.4$ & $47.4612 \pm 0.0420$ & $8.649 \pm 0.022$ & $0.452 \pm 0.046$ & & & Subdwarfs? \\
\hline Gl 380 & 7.24 & 0.801 & 4002 & 4003 & $4002 \pm 1$ & $\mathrm{dK} 7.5$ & $205.3917 \pm 0.0342$ & $8.803 \pm 0.020$ & $0.503 \pm 0.010$ & & & \\
\hline Gl 389.1 & 9.99 & 0.692 & 4275 & - & $4275 \pm 41$ & $\mathrm{dK} 6.0$ & $34.3613 \pm 0.0623$ & $7.670 \pm 0.024$ & $0.667 \pm 0.034$ & & & \\
\hline Gl 397 & 8.83 & 0.743 & 4146 & 4181 & $4163 \pm 18$ & dK6.6 & $62.4489 \pm 0.0595$ & $7.808 \pm 0.022$ & $0.689 \pm 0.024$ & +0.04 & & \\
\hline Gl 397.1 & 9.674 & 0.781 & 4050 & - & $4050 \pm 41$ & $\mathrm{dK} 6.1$ & $56.9607 \pm 0.0309$ & $8.452 \pm 0.021$ & $0.566 \pm 0.032$ & & & \\
\hline Gl 401 & 11.02 & 0.775 & 4066 & - & $4066 \pm 41$ & sdK7.2 & $53.1593 \pm 0.0570$ & $9.648 \pm 0.022$ & $0.322 \pm 0.019$ & -0.55 & & \\
\hline Gl $412.3 \mathrm{~A}$ & 10.088 & 0.695 & 4268 & 4201 & $4234 \pm 33$ & $\mathrm{dK} 6.2$ & $36.8377 \pm 0.3025$ & $7.919 \pm 0.038$ & $0.614 \pm 0.034$ & & & \\
\hline Gl $412.3 \mathrm{~B}$ & 10.088 & 0.695 & 4268 & 4201 & $4234 \pm 33$ & $\mathrm{dK} 6.2$ & $36.8377 \pm 0.3025$ & $7.919 \pm 0.038$ & $0.614 \pm 0.034$ & & & \\
\hline Gl 414A & 8.34 & 0.776 & 4063 & 4060 & $4061 \pm 1$ & $\operatorname{sdK} 7.2$ & $84.0803 \pm 0.0471$ & $7.963 \pm 0.021$ & $0.702 \pm 0.014$ & -0.99 & 10 & Binary? \\
\hline Gl 416 & 9.038 & 0.706 & 4240 & 4276 & $4258 \pm 18$ & dK6.1 & $45.4075 \pm 0.5798$ & $7.324 \pm 0.048$ & $0.792 \pm 0.035$ & & 2 & Binary? \\
\hline Gl $421 \mathrm{~A}$ & 10.323 & 0.765 & 4091 & 3951 & $4021 \pm 70$ & sdK7.4 & $43.5608 \pm 0.0428$ & $8.518 \pm 0.022$ & $0.564 \pm 0.049$ & -1.50 & & \\
\hline Gl 421B & 10.030 & 0.788 & 4033 & 3951 & $3992 \pm 41$ & sdK7.5 & $43.5895 \pm 0.0405$ & $8.227 \pm 0.022$ & $0.663 \pm 0.044$ & -1.50 & 4 & \\
\hline Gl $425 \mathrm{~B}$ & 8.61 & 0.820 & 3975 & 3955 & $3965 \pm 10$ & $\mathrm{dK} 7.6$ & $76.4165 \pm 0.0821$ & $8.026 \pm 0.022$ & $0.749 \pm 0.023$ & +0.19 & 1 & \\
\hline Gl 430 & 9.522 & 0.781 & 4050 & 4161 & $4106 \pm 55$ & $\mathrm{dK} 7.0$ & $43.4651 \pm 0.0233$ & $7.713 \pm 0.021$ & $0.757 \pm 0.053$ & & & Binary? \\
\hline Gl 435.1 & 9.585 & 0.676 & 4316 & - & $4316 \pm 41$ & dK5.7 & $32.2570 \pm 0.2491$ & $7.128 \pm 0.037$ & $0.829 \pm 0.047$ & & & Binary? \\
\hline Gl 455.1 & 10.016 & 0.751 & 4126 & 4262 & $4194 \pm 68$ & dK6.5 & $42.7177 \pm 0.0628$ & $8.169 \pm 0.023$ & $0.567 \pm 0.047$ & +0.51 & & \\
\hline Gl 462 & 9.39 & 0.781 & 4050 & 4136 & $4093 \pm 43$ & dK7.1 & $62.6959 \pm 0.0354$ & $8.376 \pm 0.021$ & $0.564 \pm 0.033$ & +0.40 & & \\
\hline Gl 466 & 10.370 & 0.686 & 4290 & 4355 & $4322 \pm 33$ & dK5.7 & $27.2905 \pm 0.0523$ & $7.560 \pm 0.024$ & $0.677 \pm 0.030$ & -0.05 & 3 & \\
\hline Gl 485 & 9.882 & 0.651 & 4379 & 4590 & $4485 \pm 105$ & dK4.3 & $34.5216 \pm 0.0392$ & $7.572 \pm 0.022$ & $0.596 \pm 0.059$ & & & \\
\hline Gl 496.1 & 9.057 & 0.783 & 4045 & 4075 & $4060 \pm 15$ & $\mathrm{dK} 7.2$ & $61.3899 \pm 0.0523$ & $7.997 \pm 0.022$ & $0.692 \pm 0.023$ & -0.11 & 29 & \\
\hline Gl 509.1 & 9.74 & 0.764 & 4093 & 4070 & $4081 \pm 11$ & $\mathrm{dK} 7.1$ & $41.4667 \pm 0.2621$ & $7.828 \pm 0.034$ & $0.734 \pm 0.025$ & & 2 & Binary? \\
\hline Gl 517 & 9.360 & 0.708 & 4235 & 4305 & $4270 \pm 35$ & dK6.0e & $48.7322 \pm 0.0587$ & $7.799 \pm 0.023$ & $0.630 \pm 0.034$ & -0.11 & 6 & \\
\hline Gl 522 & 9.710 & 0.712 & 4225 & 4136 & $4180 \pm 44$ & dK6.5 & $40.4528 \pm 0.0689$ & $7.745 \pm 0.024$ & $0.698 \pm 0.042$ & -0.22 & 7 & \\
\hline Gl 524.1 & 10.480 & 0.742 & 4149 & 4544 & $4346 \pm 197$ & $\mathrm{dK} 5.5$ & $33.9940 \pm 0.0744$ & $8.137 \pm 0.025$ & $0.509 \pm 0.094$ & -0.22 & 2 & \\
\hline Gl 542.2 & 9.112 & 0.711 & 4227 & 4359 & $4293 \pm 66$ & dK5.9 & $44.3763 \pm 0.7670$ & $7.348 \pm 0.057$ & $0.763 \pm 0.067$ & +0.73 & & \\
\hline Gl 546 & 8.610 & 0.716 & 4215 & 4230 & $4222 \pm 8$ & dK6.3 & $68.8608 \pm 0.0398$ & $7.800 \pm 0.021$ & $0.656 \pm 0.016$ & -0.22 & 55 & \\
\hline Gl 550.1 & 10.88 & 0.656 & 4366 & 4400 & $4383 \pm 17$ & dK5.3 & $21.8439 \pm 0.0317$ & $7.577 \pm 0.023$ & $0.641 \pm 0.019$ & +0.01 & & \\
\hline Gl 558 & 9.58 & 0.740 & 4154 & 4328 & $4241 \pm 87$ & $\mathrm{dK} 6.2$ & $49.9000 \pm 0.0282$ & $8.071 \pm 0.021$ & $0.570 \pm 0.056$ & -0.01 & 2 & \\
\hline Gl 562 & 9.23 & 0.716 & 4215 & - & $4215 \pm 41$ & dK6.3 & $56.3208 \pm 0.0432$ & $7.983 \pm 0.022$ & $0.607 \pm 0.033$ & & 6 & \\
\hline Gl 563.1 & 9.726 & 0.731 & 4177 & 4111 & $4144 \pm 33$ & dK6.8 & $41.6898 \pm 0.0263$ & $7.826 \pm 0.021$ & $0.694 \pm 0.034$ & -1.00 & & Binary? \\
\hline Gl 570.2 & 11.110 & 0.806 & 3993 & 3984 & $3988 \pm 4$ & $\mathrm{dK} 7.5$ & $21.4987 \pm 0.5332$ & $7.772 \pm 0.073$ & $0.821 \pm 0.040$ & -1.21 & & Binary? \\
\hline Gl 571 & 10.14 & 0.716 & 4215 & 4180 & $4197 \pm 17$ & $\mathrm{dK} 6.4$ & $41.3889 \pm 0.0386$ & $8.224 \pm 0.022$ & $0.552 \pm 0.018$ & +0.29 & 8 & \\
\hline Gl 571.1 & 9.472 & 0.805 & 3995 & 4079 & $4037 \pm 42$ & $\mathrm{dK} 7.3$ & $56.0214 \pm 0.0529$ & $8.214 \pm 0.022$ & $0.639 \pm 0.038$ & +0.37 & 1 & \\
\hline Gl 576 & 9.815 & 0.748 & 4134 & 4246 & $4190 \pm 56$ & dK6.5 & $52.5873 \pm 0.0668$ & $8.419 \pm 0.023$ & $0.507 \pm 0.036$ & -0.25 & & \\
\hline Gl 583 & 9.459 & 0.721 & 4202 & 4219 & $4210 \pm 9$ & dK6.4 & $52.1506 \pm 0.0532$ & $8.045 \pm 0.022$ & $0.592 \pm 0.015$ & +0.37 & 3 & \\
\hline Gl 585.1 & 10.23 & 0.743 & 4146 & 4205 & $4175 \pm 30$ & dK6.6 & $35.3151 \pm 0.0428$ & $7.970 \pm 0.023$ & $0.632 \pm 0.029$ & & & \\
\hline Gl 596A & 10.716 & 0.682 & 4301 & 4220 & $4261 \pm 41$ & $\mathrm{dK} 6.1$ & $23.1657 \pm 0.0312$ & $7.540 \pm 0.023$ & $0.715 \pm 0.036$ & -0.57 & & \\
\hline Gl 600 & 9.363 & 0.818 & 3977 & 4087 & $4032 \pm 55$ & $\mathrm{dK} 7.3$ & $47.2860 \pm 0.1747$ & $7.737 \pm 0.028$ & $0.800 \pm 0.060$ & +0.73 & 13 & Binary? \\
\hline Gl 605 & 10.473 & 0.694 & 4270 & 4394 & $4332 \pm 38$ & dK5.6 & $30.5321 \pm 0.0277$ & $7.897 \pm 0.022$ & $0.575 \pm 0.027$ & -0.31 & & \\
\hline Gl 608 & 9.983 & 0.700 & 4255 & 4276 & $4266 \pm 10$ & $\mathrm{dK} 6.0$ & $30.9793 \pm 0.0186$ & $7.438 \pm 0.021$ & $0.747 \pm 0.018$ & +0.35 & & \\
\hline Gl 619 & 8.98 & 0.746 & 4139 & 4089 & $4114 \pm 25$ & dK6.9 & $63.3463 \pm 0.0281$ & $7.989 \pm 0.021$ & $0.662 \pm 0.027$ & -0.24 & 6 & \\
\hline Gl 626 & 8.85 & 0.651 & 4379 & 4408 & $4393 \pm 15$ & dK5.3 & $57.0479 \pm 0.0414$ & $7.631 \pm 0.022$ & $0.621 \pm 0.017$ & +0.29 & & \\
\hline Gl 629.1 & 10.53 & 0.754 & 4119 & - & $4119 \pm 41$ & dK6.9 & $31.5333 \pm 0.0436$ & $8.024 \pm 0.023$ & $0.648 \pm 0.037$ & & 3 & \\
\hline Gl 630 & 11.007 & 0.768 & 4083 & 4116 & $4099 \pm 17$ & $\mathrm{dK} 7.0$ & $24.7018 \pm 0.0239$ & $7.971 \pm 0.022$ & $0.676 \pm 0.023$ & & & \\
\hline Gl 659B & 9.36 & 0.645 & 4394 & 4436 & $4415 \pm 21$ & dK5.1 & $47.0737 \pm 0.0244$ & $7.724 \pm 0.021$ & $0.585 \pm 0.019$ & & & Subdwarf? \\
\hline Gl 673 & 7.530 & 0.760 & 4104 & 4096 & $4100 \pm 4$ & $\mathrm{dK} 7.0$ & $129.6231 \pm 0.0286$ & $8.093 \pm 0.020$ & $0.638 \pm 0.014$ & +0.01 & 64 & \\
\hline Gl 697.1 & 10.155 & 0.756 & 4114 & 4069 & $4092 \pm 23$ & $\mathrm{dK} 7.1$ & $39.7152 \pm 0.0424$ & $8.150 \pm 0.022$ & $0.627 \pm 0.025$ & & & \\
\hline Gl 708 & 10.020 & 0.694 & 4270 & 4244 & $4257 \pm 13$ & dK6.1 & $34.2819 \pm 0.0371$ & $7.695 \pm 0.022$ & $0.668 \pm 0.018$ & & & \\
\hline
\end{tabular}


Table 2:: continued.

\begin{tabular}{|c|c|c|c|c|c|c|c|c|c|c|c|c|}
\hline Star & $\begin{array}{c}\mathrm{V} \\
(\mathrm{mag})\end{array}$ & $\begin{array}{c}(\mathrm{R}-\mathrm{I})_{C} \\
(\mathrm{mag})\end{array}$ & $\begin{array}{c}T_{\text {eff }}{ }^{1} \\
(\mathrm{~K})\end{array}$ & $\begin{array}{c}T_{e f f}{ }^{2} \\
(\mathrm{~K})\end{array}$ & $\begin{array}{c}T_{\text {eff }} \pm 3 \sigma^{3} \\
(\mathrm{~K})\end{array}$ & $\begin{array}{l}\text { Spect. } \\
\text { Type }\end{array}$ & $\begin{array}{c}\pi \\
(\mathrm{m} ")\end{array}$ & $\begin{array}{c}M_{V} \\
(\mathrm{mag})\end{array}$ & $\begin{array}{c}R_{\star} \\
\left(R_{\odot}\right)\end{array}$ & {$[\mathrm{M} / \mathrm{H}]$} & $\begin{array}{l}\text { Hi. Res. } \\
\text { Spectra }\end{array}$ & Comments \\
\hline Gl 710 & 9.660 & 0.755 & 4116 & 4130 & $4123 \pm 7$ & dK6.9 & $52.5185 \pm 0.0478$ & $8.262 \pm 0.022$ & $0.579 \pm 0.014$ & +0.00 & 4 & \\
\hline Gl 726 & 8.810 & 0.700 & 4255 & 4208 & $4231 \pm 23$ & sdK6.2 & $70.1428 \pm 0.0609$ & $8.040 \pm 0.022$ & $0.583 \pm 0.022$ & -0.97 & 20 & \\
\hline Gl 728 & 9.155 & 0.776 & 4063 & 3951 & $4007 \pm 56$ & sdK7.4 & $59.9538 \pm 0.0428$ & $8.044 \pm 0.022$ & $0.710 \pm 0.052$ & -1.50 & 47 & Binary? \\
\hline Gl 747.2 & 9.73 & 0.743 & 4146 & 4183 & $4164 \pm 19$ & dK6.6 & $34.7890 \pm 0.3861$ & $7.437 \pm 0.044$ & $0.816 \pm 0.037$ & & & Binary? \\
\hline Gl 747.3 & 9.342 & 0.730 & 4179 & 4170 & $4174 \pm 4$ & dK6.6 & $48.1310 \pm 0.2524$ & $7.754 \pm 0.031$ & $0.699 \pm 0.018$ & +0.33 & 6 & \\
\hline Gl $748.2 B$ & 11.094 & 0.807 & 3992 & - & $3992 \pm 41$ & $\mathrm{dK} 7.5$ & $37.2329 \pm 0.0411$ & $8.949 \pm 0.022$ & $0.475 \pm 0.032$ & -0.13 & & \\
\hline Gl 757 & 10.95 & 0.802 & 3999 & 4100 & $4049 \pm 51$ & $\operatorname{sdK} 7.2$ & $36.1947 \pm 0.0624$ & $8.743 \pm 0.024$ & $0.495 \pm 0.034$ & & 6 & Subdwarf? \\
\hline Gl 763 & 9.326 & 0.790 & 4028 & 4011 & $4019 \pm 7$ & sdK7.4 & $69.3122 \pm 0.0389$ & $8.530 \pm 0.021$ & $0.562 \pm 0.014$ & -0.88 & 8 & \\
\hline Gl 773 & 9.285 & 0.737 & 4162 & 4396 & $4279 \pm 117$ & dK5.9 & $56.2226 \pm 0.0974$ & $8.035 \pm 0.024$ & $0.562 \pm 0.064$ & -0.10 & & Subdwarf? \\
\hline Gl 782 & 8.890 & 0.691 & 4278 & 4377 & $4327 \pm 50$ & sdK5.7 & $63.2814 \pm 0.0714$ & $7.896 \pm 0.022$ & $0.557 \pm 0.033$ & -0.88 & 4 & \\
\hline Gl 786.1 & 10.16 & 0.800 & 4002 & 4112 & $4057 \pm 55$ & $\mathrm{dK} 7.2$ & $39.5368 \pm 0.0365$ & $8.145 \pm 0.022$ & $0.648 \pm 0.046$ & & 2 & \\
\hline Gl 795A & 9.348 & 0.707 & 4237 & 4148 & $4192 \pm 44$ & dK6.5 & $59.80 \pm 3.42$ & $8.232 \pm 0.141$ & $0.552 \pm 0.065$ & & 1 & Subdwarf? \\
\hline Gl 795B & 9.348 & 0.707 & 4237 & 4148 & $4192 \pm 44$ & dK6.5 & $59.80 \pm 3.42$ & $8.232 \pm 0.141$ & $0.552 \pm 0.065$ & & 1 & Subdwarf? \\
\hline Gl 798 & 8.82 & 0.748 & 4134 & 4250 & $4192 \pm 58$ & sdK6.5 & $81.1200 \pm 0.0480$ & $8.366 \pm 0.021$ & $0.519 \pm 0.037$ & -1.50 & 5 & \\
\hline Gl 801 & 9.88 & 0.704 & 4245 & - & $4245 \pm 41$ & $\mathrm{dK} 6.2$ & $38.4592 \pm 0.0530$ & $7.805 \pm 0.023$ & $0.641 \pm 0.036$ & -0.21 & 1 & \\
\hline Gl $820 \mathrm{~B}$ & 7.40 & 0.785 & 4040 & - & $4040 \pm 41$ & $\operatorname{sdK} 7.3$ & $286.1457 \pm 0.0590$ & $9.683 \pm 0.020$ & $0.324 \pm 0.018$ & & & Subdwarf? \\
\hline Gl 826.1 & 9.086 & 0.749 & 4131 & 4350 & $4240 \pm 110$ & dK6.2 & $63.3493 \pm 0.0899$ & $8.095 \pm 0.023$ & $0.564 \pm 0.069$ & & 3 & \\
\hline Gl 828.1 & 10.480 & 0.803 & 3997 & 4010 & $4003 \pm 7$ & $\mathrm{dK} 7.5$ & $36.0460 \pm 0.0387$ & $8.264 \pm 0.022$ & $0.644 \pm 0.017$ & +0.93 & 2 & \\
\hline Gl 830 & 9.075 & 0.728 & 4184 & 4124 & $4154 \pm 30$ & dK6.7 & $56.4251 \pm 0.0600$ & $7.832 \pm 0.022$ & $0.686 \pm 0.032$ & -0.21 & 5 & \\
\hline Gl $836.9 \mathrm{~A}$ & 9.60 & 0.775 & 4066 & 4130 & $4098 \pm 32$ & $\mathrm{dK} 7.0$ & $52.1 \pm 8.5$ & $8.184 \pm 0.348$ & $0.613 \pm 0.134$ & & & \\
\hline Gl $836.9 B$ & 9.60 & 0.775 & 4066 & 4130 & $4098 \pm 32$ & $\mathrm{dK} 7.0$ & $52.1 \pm 8.5$ & $8.184 \pm 0.348$ & $0.613 \pm 0.134$ & & & \\
\hline Gl $847 \mathrm{~A}$ & 10.97 & 0.761 & 4101 & - & $4101 \pm 41$ & $\mathrm{dK} 7.0$ & $29.6910 \pm 0.0238$ & $8.333 \pm 0.022$ & $0.571 \pm 0.032$ & & & \\
\hline Gl 857.1 & 8.770 & 0.728 & 4184 & 4074 & $4129 \pm 55$ & dK6.9 & $47.9000 \pm 0.0927$ & $7.172 \pm 0.024$ & $0.951 \pm 0.068$ & & 2 & Binary? \\
\hline Gl 883 & 10.568 & 0.811 & 3987 & 4023 & $4005 \pm 18$ & $\mathrm{dK} 7.4$ & $38.4083 \pm 0.0498$ & $8.490 \pm 0.023$ & $0.579 \pm 0.021$ & +0.60 & & \\
\hline Gl 884 & 7.869 & 0.770 & 4078 & 3999 & $4038 \pm 39$ & sdK7.3 & $121.4918 \pm 0.0507$ & $8.292 \pm 0.021$ & $0.616 \pm 0.034$ & -1.16 & 17 & \\
\hline Gl 885A & 9.57 & 0.749 & 4131 & 4257 & $4194 \pm 63$ & dK6.5 & $31.4730 \pm 0.0610$ & $7.060 \pm 0.024$ & $0.945 \pm 0.074$ & & 4 & Binary? \\
\hline Gl $889 A$ & 9.640 & 0.756 & 4114 & 4251 & $4182 \pm 69$ & dK6.5 & $45.2440 \pm 0.0528$ & $7.918 \pm 0.023$ & $0.644 \pm 0.053$ & +0.74 & 4 & \\
\hline Gl 894 & 10.38 & 0.728 & 4184 & 4160 & $4172 \pm 12$ & $\mathrm{dK} 6.6$ & $30.2129 \pm 0.0571$ & $7.781 \pm 0.024$ & $0.692 \pm 0.021$ & -0.23 & 2 & \\
\hline Gl 895.3 & 10.19 & 0.691 & 4278 & 4234 & $4256 \pm 22$ & dK6.1 & $34.2247 \pm 0.0467$ & $7.862 \pm 0.023$ & $0.619 \pm 0.022$ & +0.13 & 4 & \\
\hline Gl 898 & 8.607 & 0.716 & 4215 & 4164 & $4189 \pm 25$ & $\mathrm{dK} 6.5$ & $68.6979 \pm 0.0581$ & $7.792 \pm 0.022$ & $0.678 \pm 0.028$ & +0.01 & 7 & \\
\hline Gl 900 & 9.546 & 0.814 & 3983 & 4109 & $4046 \pm 63$ & $\mathrm{dK} 7.3$ & $48.0331 \pm 0.0818$ & $7.954 \pm 0.024$ & $0.715 \pm 0.057$ & +0.05 & 5 & \\
\hline Gl 906 & 9.932 & 0.776 & 4063 & 4269 & $4166 \pm 103$ & dK6.6 & $50.4494 \pm 0.0522$ & $8.446 \pm 0.022$ & $0.512 \pm 0.060$ & +0.61 & 3 & \\
\hline Gl 907.1 & 9.63 & 0.774 & 4068 & - & $4068 \pm 41$ & $\mathrm{dK} 7.2$ & $35.4713 \pm 0.8994$ & $7.379 \pm 0.074$ & $0.913 \pm 0.076$ & & & Binary? \\
\hline HIP 1055 & 10.435 & 0.719 & 4207 & 4421 & $4314 \pm 107$ & $\mathrm{dK} 5.7$ & $24.0695 \pm 0.1164$ & $7.342 \pm 0.030$ & $0.753 \pm 0.082$ & -0.31 & & \\
\hline HIP $3121 \mathrm{~A}$ & 11.241 & 0.742 & 4149 & 4263 & $4206 \pm 57$ & sdK6.4 & $29.4884 \pm 0.0488$ & $8.589 \pm 0.024$ & $0.463 \pm 0.033$ & -0.55 & & \\
\hline HIP 3121B & 11.241 & 0.742 & 4149 & 4263 & $4206 \pm 57$ & $\mathrm{sdK} 6.4$ & $29.4884 \pm 0.0488$ & $8.589 \pm 0.024$ & $0.463 \pm 0.033$ & -0.55 & & \\
\hline HIP 3428 & 10.97 & 0.793 & 4020 & 3971 & $3996 \pm 24$ & $\mathrm{dK} 7.5$ & $22.1804 \pm 0.0434$ & $7.700 \pm 0.024$ & $0.841 \pm 0.040$ & -0.37 & & Binary? \\
\hline HIP $5110 \mathrm{~A}$ & 9.26 & 0.645 & 4394 & 4305 & $4350 \pm 45$ & dK5.5 & $36.6470 \pm 0.0432$ & $7.080 \pm 0.023$ & $0.826 \pm 0.043$ & -0.09 & & Binary? \\
\hline HIP 5110B & 9.71 & 0.645 & 4394 & 4305 & $4350 \pm 45$ & dK5.5 & $37.4109 \pm 0.1135$ & $7.575 \pm 0.027$ & $0.658 \pm 0.036$ & -0.09 & & \\
\hline HIP 6239 & 11.295 & 0.743 & 4146 & - & $4146 \pm 41$ & $\mathrm{dK} 6.7$ & $23.0156 \pm 0.0471$ & $8.105 \pm 0.024$ & $0.610 \pm 0.035$ & & & \\
\hline HIP 6290 & 10.39 & 0.743 & 4146 & 4200 & $4173 \pm 27$ & $\mathrm{dK} 6.6$ & $45.9702 \pm 0.0373$ & $8.702 \pm 0.022$ & $0.452 \pm 0.019$ & +0.42 & & \\
\hline HIP 7420 & 10.985 & 0.670 & 4331 & 4382 & $4357 \pm 25$ & dK5.5 & $21.7609 \pm 0.0646$ & $7.673 \pm 0.026$ & $0.626 \pm 0.024$ & -0.21 & & \\
\hline HIP 7817 & 11.535 & 0.706 & 4240 & 4049 & $4145 \pm 95$ & $\mathrm{dK} 6.8$ & $21.7976 \pm 0.1358$ & $8.227 \pm 0.033$ & $0.577 \pm 0.066$ & & & \\
\hline HIP 9067 & 11.431 & 0.756 & 4114 & 4082 & $4098 \pm 16$ & $\mathrm{dK} 7.0$ & $20.9876 \pm 0.0388$ & $8.041 \pm 0.024$ & $0.655 \pm 0.022$ & & & \\
\hline HIP 10960 & 10.34 & 0.694 & 4270 & 4297 & $4284 \pm 13$ & dK5.9 & $34.7029 \pm 0.1180$ & $8.042 \pm 0.027$ & $0.558 \pm 0.017$ & +0.52 & & \\
\hline HIP 11437 & 10.19 & 0.667 & 4338 & 4323 & $4330 \pm 7$ & dK5.6 & $24.3599 \pm 0.0496$ & $7.123 \pm 0.024$ & $0.822 \pm 0.019$ & & 1 & Binary? \\
\hline HIP 11707 & 10.912 & 0.731 & 4177 & 4194 & $4186 \pm 8$ & sdK6.5 & $27.7059 \pm 0.0453$ & $8.125 \pm 0.024$ & $0.583 \pm 0.015$ & -1.66 & & \\
\hline HIP 13323 & 10.895 & 0.706 & 4240 & 4213 & $4227 \pm 14$ & dK6.3 & $21.7240 \pm 0.0501$ & $7.580 \pm 0.025$ & $0.723 \pm 0.023$ & +0.31 & & \\
\hline
\end{tabular}


Table 2:: continued.

\begin{tabular}{|c|c|c|c|c|c|c|c|c|c|c|c|c|}
\hline Star & $\begin{array}{c}\mathrm{V} \\
(\mathrm{mag})\end{array}$ & $\begin{array}{c}(\mathrm{R}-\mathrm{I})_{C} \\
(\mathrm{mag})\end{array}$ & $\begin{array}{c}T_{e f f}{ }^{1} \\
(\mathrm{~K})\end{array}$ & $\begin{array}{c}T_{e f f}^{2} \\
(\mathrm{~K})\end{array}$ & $T_{\text {eff }} \pm 3 \sigma^{3}$ & $\begin{array}{l}\text { Spect. } \\
\text { Type }\end{array}$ & $\begin{array}{c}\pi \\
(\mathrm{m} ")\end{array}$ & $\begin{array}{c}M_{V} \\
\text { (mag) }\end{array}$ & $\begin{array}{c}R_{\star} \\
\left(R_{\odot}\right)\end{array}$ & {$[\mathrm{M} / \mathrm{H}]$} & $\begin{array}{l}\text { Hi. Res. } \\
\text { Spectra }\end{array}$ & Comments \\
\hline HIP $14593 \mathrm{~A}$ & 10.06 & 0.716 & 4215 & 4220 & $4217 \pm 4$ & $\mathrm{dK} 6.3$ & $34.9234 \pm 0.0386$ & $7.776 \pm 0.022$ & $0.666 \pm 0.014$ & +0.21 & 1 & \\
\hline HIP 15234 & 10.680 & 0.700 & 4255 & 4215 & $4235 \pm 20$ & sdK6.2 & $32.8499 \pm 0.0572$ & $8.263 \pm 0.024$ & $0.524 \pm 0.019$ & -0.99 & & \\
\hline HIP 15466 & 10.57 & 0.719 & 4207 & 4389 & $4298 \pm 91$ & $\mathrm{dK} 5.8$ & $29.9146 \pm 0.0638$ & $7.949 \pm 0.025$ & $0.576 \pm 0.053$ & & & \\
\hline HIP 16560 & 11.230 & 0.706 & 4240 & 4303 & $4272 \pm 31$ & dK6.0 & $19.4683 \pm 0.0369$ & $7.677 \pm 0.024$ & $0.666 \pm 0.028$ & +0.12 & & \\
\hline HIP $18377 \mathrm{~A}$ & 10.927 & 0.657 & 4364 & 4302 & $4333 \pm 31$ & dK5.6 & $21.2051 \pm 0.4439$ & $7.559 \pm 0.065$ & $0.671 \pm 0.042$ & +0.19 & & \\
\hline HIP $18377 \mathrm{~B}$ & 11.005 & 0.657 & 4364 & 4302 & $4333 \pm 31$ & dK5.6 & $26.9176 \pm 0.4970$ & $8.155 \pm 0.060$ & $0.510 \pm 0.030$ & +0.19 & & Subdwarf? \\
\hline HIP 19082 & 11.41 & 0.731 & 4177 & 4039 & $4108 \pm 69$ & $\mathrm{dK} 7.0$ & $21.1435 \pm 0.0517$ & $8.036 \pm 0.025$ & $0.651 \pm 0.056$ & & 1 & \\
\hline HIP 19316 & 11.275 & 0.731 & 4177 & - & $4177 \pm 41$ & dK6.6 & $21.5318 \pm 0.0694$ & $7.940 \pm 0.027$ & $0.640 \pm 0.037$ & & 1 & \\
\hline HIP 19410A & 11.39 & 0.704 & 4245 & 4387 & $4316 \pm 71$ & dK5.7 & $24.6701 \pm 0.0381$ & $8.351 \pm 0.023$ & $0.472 \pm 0.035$ & +0.01 & & Subdwarf? \\
\hline HIP $19410 \mathrm{~B}$ & 12.15 & 0.704 & 4245 & 4387 & $4316 \pm 71$ & dK5.7 & $24.6701 \pm 0.0381$ & $9.111 \pm 0.023$ & $0.333 \pm 0.025$ & & & Subdwarf? \\
\hline HIP $19410 \mathrm{C}$ & 12.15 & 0.704 & 4245 & 4387 & $4316 \pm 71$ & dK5.7 & $24.6701 \pm 0.0381$ & $9.111 \pm 0.023$ & $0.333 \pm 0.025$ & & & Subdwarf? \\
\hline HIP 19808A & 11.454 & 0.670 & 4331 & - & $4331 \pm 41$ & dK5.6 & $22.4472 \pm 0.0916$ & $8.210 \pm 0.029$ & $0.498 \pm 0.026$ & & 1 & Subdwarf? \\
\hline HIP $19808 \mathrm{~B}$ & 11.454 & 0.670 & 4331 & - & $4331 \pm 41$ & dK5.6 & $22.4472 \pm 0.0916$ & $8.210 \pm 0.029$ & $0.498 \pm 0.026$ & & 1 & Subdwarf? \\
\hline HIP 19834 & 11.55 & 0.756 & 4114 & - & $4114 \pm 41$ & $\mathrm{dK} 6.9$ & $20.8276 \pm 0.0383$ & $8.143 \pm 0.024$ & $0.616 \pm 0.036$ & & 1 & \\
\hline HIP 20012 & 11.378 & 0.713 & 4222 & - & $4222 \pm 41$ & $\mathrm{dK} 6.3$ & $27.1039 \pm 0.0596$ & $8.543 \pm 0.025$ & $0.466 \pm 0.026$ & & & Subdwarf? \\
\hline HIP 20142 & 10.581 & 0.782 & 4048 & 4002 & $4024 \pm 22$ & $\mathrm{dK} 7.4$ & $34.1459 \pm 0.0380$ & $8.248 \pm 0.022$ & $0.637 \pm 0.025$ & & & \\
\hline HIP 20745 & 10.438 & 0.768 & 4083 & 4088 & $4085 \pm 3$ & $\mathrm{dK} 7.1$ & $29.09 \pm 2.68$ & $7.757 \pm 0.211$ & $0.756 \pm 0.086$ & & 1 & Binary? \\
\hline HIP $20895 \mathrm{~A}$ & 11.726 & 0.781 & 4050 & 4170 & $4110 \pm 60$ & $\mathrm{dK} 7.0$ & $20.7793 \pm 0.3401$ & $8.314 \pm 0.055$ & $0.572 \pm 0.052$ & & & \\
\hline HIP 20895B & 11.726 & 0.781 & 4050 & 4170 & $4110 \pm 60$ & $\mathrm{dK} 7.0$ & $20.7793 \pm 0.3401$ & $8.314 \pm 0.055$ & $0.572 \pm 0.052$ & & & \\
\hline HIP 21006 & 10.470 & 0.805 & 3995 & 3933 & $3964 \pm 31$ & $\mathrm{dK} 7.6$ & $37.4062 \pm 0.0557$ & $8.335 \pm 0.023$ & $0.650 \pm 0.037$ & & 2 & \\
\hline HIP 21138 & 10.974 & 0.670 & 4331 & 4365 & $4348 \pm 17$ & dK5.5 & $21.8367 \pm 0.0378$ & $7.670 \pm 0.024$ & $0.631 \pm 0.019$ & +0.09 & 1 & \\
\hline HIP $21659 \mathrm{~A}$ & 12.01 & 0.657 & 4364 & 4545 & $4455 \pm 90$ & $\mathrm{dK} 4.8$ & $14.0122 \pm 0.0485$ & $7.743 \pm 0.028$ & $0.563 \pm 0.051$ & & & Subdwarf? \\
\hline HIP 21659B & 12.21 & 0.657 & 4364 & 4545 & $4455 \pm 90$ & $\mathrm{dK} 4.8$ & $14.0514 \pm 0.0497$ & $7.949 \pm 0.028$ & $0.512 \pm 0.046$ & & & Subdwarf? \\
\hline HIP 21865 & 10.03 & 0.680 & 4306 & 4304 & $4305 \pm 1$ & dK5.8 & $31.9499 \pm 0.0220$ & $7.552 \pm 0.021$ & $0.688 \pm 0.012$ & -0.19 & 2 & \\
\hline HIP 21912 & 11.229 & 0.700 & 4255 & - & $4255 \pm 41$ & $\mathrm{dK} 6.1$ & $22.5151 \pm 0.0676$ & $7.991 \pm 0.027$ & $0.584 \pm 0.030$ & & & \\
\hline HIP 21946 & 9.863 & 0.682 & 4301 & 4361 & $4330 \pm 31$ & dK5.6 & $23.4888 \pm 0.2356$ & $6.717 \pm 0.042$ & $0.992 \pm 0.050$ & & 12 & Binary? \\
\hline HIP 22177 & 10.92 & 0.657 & 4363 & 4351 & $4357 \pm 6$ & dK5.5 & $22.1438 \pm 0.0414$ & $7.646 \pm 0.024$ & $0.633 \pm 0.014$ & & 1 & \\
\hline HIP 23113 & 11.75 & 0.781 & 4050 & 4207 & $4129 \pm 78$ & dK6.9 & $26.0447 \pm 0.0612$ & $8.829 \pm 0.025$ & $0.443 \pm 0.042$ & -0.31 & & Subdwarf? \\
\hline HIP 23198 & 10.84 & 0.682 & 4300 & 4175 & $4238 \pm 62$ & $\mathrm{dK} 6.2$ & $25.8373 \pm 0.0452$ & $7.901 \pm 0.024$ & $0.617 \pm 0.047$ & & & \\
\hline HIP 28494 & 10.055 & 0.645 & 4394 & 4315 & $4355 \pm 39$ & dK5.5 & $34.3277 \pm 0.0533$ & $7.733 \pm 0.023$ & $0.609 \pm 0.029$ & +0.52 & & \\
\hline HIP 28736 & 11.030 & 0.700 & 4255 & 4280 & $4268 \pm 12$ & sdK6.0 & $20.3386 \pm 0.0497$ & $7.572 \pm 0.025$ & $0.701 \pm 0.020$ & -0.81 & & Binary? \\
\hline HIP 29036 & 10.645 & 0.700 & 4255 & 4353 & $4304 \pm 49$ & dK5.8 & $26.3007 \pm 0.0249$ & $7.745 \pm 0.022$ & $0.630 \pm 0.035$ & & & \\
\hline HIP 29132 & 10.40 & 0.642 & 4401 & 4274 & $4337 \pm 63$ & dK5.6 & $28.5209 \pm 0.0595$ & $7.676 \pm 0.025$ & $0.634 \pm 0.043$ & & 2 & \\
\hline HIP 29875 & 10.30 & 0.682 & 4300 & 4343 & $4322 \pm 21$ & dK5.7 & $32.3503 \pm 0.0447$ & $7.849 \pm 0.023$ & $0.592 \pm 0.020$ & & & Subdwarf? \\
\hline HIP 32754 & 10.29 & 0.657 & 4363 & 4405 & $4384 \pm 21$ & dK5.3 & $28.8284 \pm 0.0392$ & $7.589 \pm 0.023$ & $0.637 \pm 0.021$ & +0.07 & & \\
\hline HIP 33368 & 10.913 & 0.682 & 4300 & 4312 & $4306 \pm 6$ & dK5.8 & $21.2394 \pm 0.1657$ & $7.549 \pm 0.037$ & $0.689 \pm 0.020$ & +0.48 & & \\
\hline HIP 38492 & 10.500 & 0.768 & 4083 & 4105 & $4094 \pm 11$ & $\mathrm{dK} 7.0$ & $29.3789 \pm 0.0401$ & $7.840 \pm 0.023$ & $0.721 \pm 0.021$ & & & Binary? \\
\hline HIP 40140 & 11.348 & 0.657 & 4363 & 4389 & $4376 \pm 13$ & dK5.4 & $19.0635 \pm 0.0443$ & $7.749 \pm 0.025$ & $0.596 \pm 0.017$ & & & Subdwarf? \\
\hline HIP 40170 & 10.14 & 0.706 & 4240 & 4390 & $4315 \pm 75$ & dK5.7 & $36.6005 \pm 0.0365$ & $7.957 \pm 0.022$ & $0.567 \pm 0.044$ & +0.12 & & Subdwarf? \\
\hline HIP 41626 & 11.143 & 0.657 & 4363 & 4333 & $4348 \pm 15$ & dK5.5 & $20.0205 \pm 0.0361$ & $7.650 \pm 0.024$ & $0.637 \pm 0.019$ & +0.66 & & \\
\hline HIP $42108 \mathrm{~A}$ & 11.03 & 0.716 & 4215 & 4346 & $4280 \pm 66$ & dK5.9 & $28.9916 \pm 0.1167$ & $8.341 \pm 0.029$ & $0.488 \pm 0.036$ & & & Subdwarf? \\
\hline HIP 42108B & 11.03 & 0.716 & 4215 & 4368 & $4291 \pm 77$ & dK5.9 & $28.9916 \pm 0.1167$ & $8.341 \pm 0.029$ & $0.483 \pm 0.040$ & & & Subdwarf? \\
\hline HIP 42910 & 10.16 & 0.752 & 4124 & 4060 & $4092 \pm 32$ & $\mathrm{dK} 7.1$ & $27.4142 \pm 0.1383$ & $7.350 \pm 0.031$ & $0.906 \pm 0.048$ & +0.16 & & Binary? \\
\hline HIP 43233 & 10.345 & 0.768 & 4083 & 4214 & $4148 \pm 66$ & $\mathrm{dK} 6.7$ & $35.2183 \pm 0.0370$ & $8.079 \pm 0.022$ & $0.616 \pm 0.050$ & -0.54 & 1 & \\
\hline HIP 43274 & 10.955 & 0.756 & 4114 & 4072 & $4093 \pm 21$ & $\mathrm{dK} 7.1$ & $26.5331 \pm 0.0483$ & $8.074 \pm 0.024$ & $0.648 \pm 0.025$ & & & \\
\hline HIP $44295 \mathrm{~A}$ & 10.09 & 0.706 & 4240 & 4250 & $4245 \pm 5$ & sdK6.2 & $54.1310 \pm 0.0416$ & $8.757 \pm 0.022$ & $0.414 \pm 0.009$ & & & Subdwarf? \\
\hline HIP $44295 \mathrm{~B}$ & 10.27 & 0.706 & 4240 & 4250 & $4245 \pm 5$ & sdK6.2 & $54.2762 \pm 0.0528$ & $8.943 \pm 0.022$ & $0.380 \pm 0.008$ & & & Subdwarf? \\
\hline HIP 46493 & 10.445 & 0.700 & 4255 & 4263 & $4259 \pm 4$ & $\mathrm{dK} 6.1$ & $29.9328 \pm 0.0532$ & $7.826 \pm 0.024$ & $0.628 \pm 0.013$ & & & \\
\hline HIP 47261 & 9.80 & 0.645 & 4394 & 4223 & $4309 \pm 85$ & dK5.8 & $32.1443 \pm 0.0612$ & $7.336 \pm 0.024$ & $0.758 \pm 0.066$ & & & \\
\hline HIP 48016 & 10.949 & 0.756 & 4114 & 4378 & $4246 \pm 132$ & sdK6.1 & $33.3877 \pm 0.0645$ & $8.567 \pm 0.024$ & $0.451 \pm 0.066$ & -1.80 & & \\
\hline HIP 50213 & 12.53 & 0.677 & 4313 & - & $4313 \pm 41$ & dK5.7 & $13.4741 \pm 0.0483$ & $8.177 \pm 0.028$ & $0.513 \pm 0.027$ & & & Subdwarf? \\
\hline HIP 50375 & 10.900 & 0.719 & 4207 & 4073 & $4140 \pm 67$ & dK6.8 & $26.9554 \pm 0.0647$ & $8.053 \pm 0.025$ & $0.628 \pm 0.052$ & & & \\
\hline
\end{tabular}


Table 2:: continued.

\begin{tabular}{|c|c|c|c|c|c|c|c|c|c|c|c|c|}
\hline Star & $\underset{(\mathrm{mag})}{\mathrm{V}}$ & $\begin{array}{c}(\mathrm{R}-\mathrm{I})_{C} \\
(\mathrm{mag})\end{array}$ & $\begin{array}{c}T_{\text {eff }}{ }^{1} \\
(\mathrm{~K})\end{array}$ & $\begin{array}{c}T_{e f f}^{2} \\
(\mathrm{~K})\end{array}$ & $T_{\text {eff }} \pm 3 \sigma^{3}$ & $\begin{array}{l}\text { Spect. } \\
\text { Type }\end{array}$ & $\begin{array}{c}\pi \\
(\mathrm{m} ")\end{array}$ & $\begin{array}{c}M_{V} \\
(\mathrm{mag})\end{array}$ & $\begin{array}{c}R_{\star} \\
\left(R_{\odot}\right)\end{array}$ & {$[\mathrm{M} / \mathrm{H}]$} & $\begin{array}{l}\text { Hi. Res. } \\
\text { Spectra }\end{array}$ & Comments \\
\hline HIP 50773 & 10.69 & 0.788 & 4033 & 4263 & $4148 \pm 115$ & dK6.7 & $33.2493 \pm 0.0469$ & $8.299 \pm 0.023$ & $0.557 \pm 0.072$ & +0.66 & 3 & \\
\hline HIP 50960 & 10.43 & 0.719 & 4207 & 4107 & $4057 \pm 50$ & $\mathrm{dK} 7.2$ & $31.8241 \pm 0.0273$ & $7.944 \pm 0.022$ & $0.711 \pm 0.047$ & +1.12 & & \\
\hline HIP 51073 & 10.83 & 0.697 & 4263 & 4305 & $4284 \pm 21$ & sdK5.9 & $24.7179 \pm 0.0638$ & $7.795 \pm 0.026$ & $0.625 \pm 0.022$ & -0.72 & 9 & \\
\hline HIP 51263A & 10.44 & 0.692 & 4275 & 4403 & $4339 \pm 64$ & dK5.6 & $27.4281 \pm 0.5896$ & $7.631 \pm 0.066$ & $0.647 \pm 0.058$ & & & \\
\hline HIP 51263B & 10.44 & 0.692 & 4275 & 4403 & $4339 \pm 64$ & dK5.6 & $27.4281 \pm 0.5896$ & $7.631 \pm 0.066$ & $0.647 \pm 0.058$ & & & \\
\hline HIP 52008 & 10.536 & 0.657 & 4363 & 4341 & $4352 \pm 11$ & dK5.5 & $24.8547 \pm 0.0385$ & $7.513 \pm 0.023$ & $0.676 \pm 0.017$ & & & \\
\hline HIP 52544 & 11.00 & 0.768 & 4083 & 4165 & $4124 \pm 41$ & dK6.9 & $26.6141 \pm 0.0285$ & $8.126 \pm 0.022$ & $0.616 \pm 0.035$ & +0.45 & & \\
\hline HIP 53175 & 10.69 & 0.788 & 4033 & 4120 & $4076 \pm 44$ & $\mathrm{dK} 7.1$ & $25.1842 \pm 0.0820$ & $7.696 \pm 0.027$ & $0.783 \pm 0.049$ & & 7 & Binary? \\
\hline HIP 54559 & 11.063 & 0.719 & 4207 & 4262 & $4235 \pm 27$ & $\mathrm{dK} 6.2$ & $24.6467 \pm 0.0326$ & $8.022 \pm 0.023$ & $0.586 \pm 0.025$ & -0.08 & & \\
\hline HIP 54697 & 10.542 & 0.806 & 3993 & 4170 & $4082 \pm 88$ & $\mathrm{dK} 7.1$ & $27.3537 \pm 0.0900$ & $7.727 \pm 0.027$ & $0.768 \pm 0.081$ & -0.12 & & Binary? \\
\hline HIP 54888 & 11.404 & 0.756 & 4114 & 4032 & $4073 \pm 41$ & $\mathrm{dK} 7.1$ & $15.3594 \pm 0.2786$ & $7.336 \pm 0.059$ & $0.927 \pm 0.070$ & & & Binary? \\
\hline HIP 54922 & 9.005 & 0.657 & 4363 & 4606 & $4485 \pm 121$ & $\mathrm{dK} 4.3$ & $43.2735 \pm 0.0473$ & $7.186 \pm 0.022$ & $0.711 \pm 0.080$ & -0.30 & & \\
\hline HIP 55988 & 10.235 & 0.676 & 4316 & 4260 & $4288 \pm 28$ & $\mathrm{sdK} 5.3$ & $35.2851 \pm 0.0550$ & $7.973 \pm 0.023$ & $0.574 \pm 0.023$ & -0.53 & & \\
\hline HIP 56715 & 10.815 & 0.704 & 4245 & 4347 & $4296 \pm 51$ & dK5.8 & $23.0116 \pm 0.0392$ & $7.625 \pm 0.024$ & $0.670 \pm 0.039$ & -0.07 & & \\
\hline HIP 56838 & 10.03 & 0.698 & 4260 & - & $4260 \pm 41$ & $\mathrm{dK} 6.1$ & $33.3875 \pm 0.0423$ & $7.648 \pm 0.023$ & $0.681 \pm 0.034$ & & 3 & \\
\hline HIP 57857 & 11.076 & 0.657 & 4363 & - & $4363 \pm 41$ & sdK5.4 & $18.4957 \pm 0.1178$ & $7.411 \pm 0.034$ & $0.703 \pm 0.038$ & -0.78 & & Binary? \\
\hline HIP 58212 & 10.961 & 0.706 & 4240 & 4001 & $4121 \pm 121$ & dK6.9 & $22.1196 \pm 0.0576$ & $7.685 \pm 0.026$ & $0.756 \pm 0.105$ & & & \\
\hline HIP 58515 & 10.762 & 0.645 & 4394 & 4386 & $4390 \pm 4$ & $\mathrm{dK} 5.3$ & $20.4808 \pm 0.1845$ & $7.319 \pm 0.039$ & $0.718 \pm 0.020$ & +0.03 & & \\
\hline HIP 58817 & 10.738 & 0.682 & 4300 & 4342 & $4324 \pm 24$ & dK5.7 & $26.7983 \pm 0.0649$ & $7.879 \pm 0.025$ & $0.584 \pm 0.022$ & +0.42 & & \\
\hline HIP 58945 & 11.12 & 0.752 & 4124 & 4187 & $4155 \pm 32$ & dK6.7 & $22.9730 \pm 0.0545$ & $7.941 \pm 0.025$ & $0.657 \pm 0.032$ & & 1 & \\
\hline HIP 59247 & 11.04 & 0.776 & 4063 & 4265 & $4164 \pm 101$ & dK6.6 & $23.833 \pm 0.261$ & $7.926 \pm 0.044$ & $0.652 \pm 0.082$ & -0.27 & 4 & \\
\hline HIP 60115 & 11.730 & 0.755 & 4116 & - & $4116 \pm 41$ & dK6.9 & $18.6754 \pm 0.0279$ & $8.086 \pm 0.023$ & $0.631 \pm 0.036$ & & & \\
\hline HIP 60186 & 11.61 & 0.731 & 4177 & 4139 & $4158 \pm 19$ & dK6.7 & $21.0389 \pm 0.0949$ & $8.225 \pm 0.030$ & $0.571 \pm 0.022$ & & & \\
\hline HIP 60265 & 10.87 & 0.700 & 4255 & 4428 & $4342 \pm 86$ & dK5.6 & $23.2440 \pm 0.0340$ & $7.702 \pm 0.023$ & $0.625 \pm 0.054$ & +0.16 & & \\
\hline HIP $60438 \mathrm{~A}$ & 11.53 & 0.781 & 4050 & 4165 & $4107 \pm 58$ & $\mathrm{dK} 7.0$ & $22.3457 \pm 0.0805$ & $8.276 \pm 0.028$ & $0.583 \pm 0.044$ & +0.06 & 1 & \\
\hline HIP $60438 \mathrm{~B}$ & 11.53 & 0.781 & 4050 & 4165 & $4107 \pm 58$ & $\mathrm{dK} 7.0$ & $22.3457 \pm 0.0805$ & $8.276 \pm 0.028$ & $0.583 \pm 0.044$ & +0.06 & 1 & \\
\hline HIP 60501 & 10.58 & 0.716 & 4215 & 4106 & $4160 \pm 54$ & dK6.7 & $17.6557 \pm 0.2917$ & $6.814 \pm 0.056$ & $1.091 \pm 0.093$ & +0.52 & 4 & PMS? \\
\hline HIP 61633 & 11.48 & 0.682 & 4300 & 4488 & $4394 \pm 94$ & dK5.3 & $17.6843 \pm 0.0278$ & $7.718 \pm 0.023$ & $0.596 \pm 0.055$ & & & \\
\hline HIP 62454 & 11.312 & 0.768 & 4083 & 3997 & $4040 \pm 43$ & $\mathrm{dK} 7.3$ & $24.1966 \pm 0.0517$ & $8.231 \pm 0.025$ & $0.632 \pm 0.039$ & & & \\
\hline HIP 62647 & 10.664 & 0.672 & 4326 & - & $4326 \pm 41$ & dK5.7 & $28.0525 \pm 0.0550$ & $7.904 \pm 0.024$ & $0.576 \pm 0.029$ & & & \\
\hline HIP 63942 & 9.398 & 0.768 & 4083 & - & $4083 \pm 41$ & $\mathrm{dK} 7.1$ & $50.9035 \pm 0.0435$ & $7.932 \pm 0.022$ & $0.698 \pm 0.040$ & & 2 & \\
\hline HIP 66828 & 10.933 & 0.717 & 4212 & 4277 & $4245 \pm 32$ & $\mathrm{dK} 6.2$ & $26.0691 \pm 0.0311$ & $8.014 \pm 0.023$ & $0.583 \pm 0.027$ & +0.50 & & \\
\hline HIP 68342 & 10.760 & 0.700 & 4255 & - & $4255 \pm 41$ & $\mathrm{dK} 6.1$ & $24.6800 \pm 0.0334$ & $7.722 \pm 0.023$ & $0.661 \pm 0.033$ & & & \\
\hline HIP 68403 & 10.266 & 0.719 & 4207 & 4369 & $4288 \pm 81$ & dK5.9 & $32.3543 \pm 0.0250$ & $7.816 \pm 0.022$ & $0.617 \pm 0.051$ & +0.32 & & \\
\hline HIP 68745 & 10.642 & 0.731 & 4177 & 3976 & $4077 \pm 101$ & $\mathrm{dK} 7.1$ & $29.4676 \pm 0.0318$ & $7.989 \pm 0.022$ & $0.684 \pm 0.080$ & +1.41 & & \\
\hline HIP $68889 \mathrm{~A}$ & 11.043 & 0.694 & 4270 & - & $4270 \pm 41$ & $\mathrm{dK} 6.0$ & $22.7018 \pm 0.0793$ & $7.823 \pm 0.028$ & $0.624 \pm 0.033$ & & & \\
\hline HIP $68889 \mathrm{~B}$ & 11.043 & 0.694 & 4270 & - & $4270 \pm 41$ & $\mathrm{dK} 6.0$ & $22.7018 \pm 0.0793$ & $7.823 \pm 0.028$ & $0.624 \pm 0.033$ & & & \\
\hline HIP 72044 & 9.91 & 0.680 & 4306 & 4365 & $4335 \pm 30$ & dK5.6 & $36.5859 \pm 0.0385$ & $7.727 \pm 0.022$ & $0.621 \pm 0.025$ & & 3 & \\
\hline HIP 72625 & 11.63 & 0.719 & 4207 & 4365 & $4286 \pm 79$ & dK5.9 & $14.8591 \pm 0.4367$ & $7.490 \pm 0.083$ & $0.718 \pm 0.080$ & -0.36 & & \\
\hline HIP 73360 & 10.62 & 0.694 & 4270 & 4286 & $4278 \pm 8$ & $\mathrm{dK} 6.0$ & $29.6140 \pm 0.0209$ & $7.977 \pm 0.022$ & $0.577 \pm 0.013$ & +0.11 & 1 & \\
\hline HIP 75116 & 11.47 & 0.676 & 4316 & 4330 & $4323 \pm 7$ & sdK5.7 & $20.7666 \pm 0.0423$ & $8.057 \pm 0.024$ & $0.538 \pm 0.013$ & -1.26 & & \\
\hline HIP 75337 & 10.527 & 0.731 & 4177 & 4204 & $4191 \pm 13$ & dK6.5 & $28.1458 \pm 0.0297$ & $7.774 \pm 0.022$ & $0.682 \pm 0.020$ & & & \\
\hline HIP 75964 & 11.66 & 0.665 & 4343 & - & $4343 \pm 41$ & dK5.6 & $18.5630 \pm 0.0412$ & $8.003 \pm 0.025$ & $0.543 \pm 0.027$ & & & Subdwarf? \\
\hline HIP 76282 & 10.816 & 0.757 & 4111 & 3985 & $4048 \pm 63$ & $\mathrm{dK} 7.3$ & $28.9336 \pm 0.0429$ & $8.123 \pm 0.023$ & $0.660 \pm 0.053$ & & & \\
\hline HIP 76374 & 11.608 & 0.756 & 4114 & 4171 & $4143 \pm 28$ & dK6.8 & $18.0642 \pm 0.0520$ & $7.892 \pm 0.026$ & $0.674 \pm 0.031$ & +0.87 & & \\
\hline HIP 76550 & 11.35 & 0.747 & 4136 & - & $4136 \pm 41$ & dK6.8 & $26.3172 \pm 0.0434$ & $8.451 \pm 0.024$ & $0.525 \pm 0.030$ & & 5 & \\
\hline HIP 76941 & 10.882 & 0.657 & 4364 & 4378 & $4371 \pm 7$ & dK5.4 & $20.1014 \pm 0.1646$ & $7.398 \pm 0.038$ & $0.703 \pm 0.021$ & & & \\
\hline HIP 77451 & 11.292 & 0.706 & 4240 & 4330 & $4285 \pm 45$ & dK5.9 & $21.3267 \pm 0.0301$ & $7.937 \pm 0.023$ & $0.585 \pm 0.031$ & & & \\
\hline HIP 78158 & 10.827 & 0.731 & 4177 & 4343 & $4260 \pm 83$ & $\mathrm{dK} 6.1$ & $19.3023 \pm 0.3945$ & $7.255 \pm 0.064$ & $0.816 \pm 0.086$ & +0.18 & & Binary? \\
\hline HIP 78395 & 10.33 & 0.716 & 4215 & 4295 & $4255 \pm 40$ & dK6.1 & $32.6057 \pm 0.0504$ & $7.896 \pm 0.023$ & $0.610 \pm 0.030$ & +0.11 & 8 & \\
\hline HIP 79419 & 9.855 & 0.694 & 4270 & 4250 & $4260 \pm 10$ & dK6.1 & $28.6792 \pm 0.0401$ & $7.143 \pm 0.023$ & $0.860 \pm 0.022$ & & & Binary? \\
\hline
\end{tabular}


Table 2:: continued.

\begin{tabular}{|c|c|c|c|c|c|c|c|c|c|c|c|c|}
\hline Star & $\begin{array}{c}\mathrm{V} \\
(\mathrm{mag})\end{array}$ & $\begin{array}{c}(\mathrm{R}-\mathrm{I})_{C} \\
(\mathrm{mag})\end{array}$ & $\begin{array}{c}T_{e f f}{ }^{1} \\
(\mathrm{~K})\end{array}$ & $\begin{array}{c}T_{e f f}^{2} \\
(\mathrm{~K})\end{array}$ & $T_{\text {eff }} \pm 3 \sigma^{3}$ & $\begin{array}{l}\text { Spect. } \\
\text { Type }\end{array}$ & $\begin{array}{c}\pi \\
(\mathrm{m} ")\end{array}$ & $\begin{array}{c}M_{V} \\
\text { (mag) }\end{array}$ & $\begin{array}{c}R_{\star} \\
\left(R_{\odot}\right)\end{array}$ & {$[\mathrm{M} / \mathrm{H}]$} & $\begin{array}{l}\text { Hi. Res. } \\
\text { Spectra }\end{array}$ & Comments \\
\hline HIP 80083 & 11.42 & 0.712 & 4225 & 4828 & $4527 \pm 301$ & sdK6.3 & $20.8594 \pm 0.0684$ & $8.017 \pm 0.027$ & $0.470 \pm 0.139$ & -0.81 & & \\
\hline HIP 80539 & 10.548 & 0.756 & 4114 & 4242 & $4178 \pm 64$ & dK6.6 & $36.4752 \pm 0.0272$ & $8.358 \pm 0.022$ & $0.527 \pm 0.041$ & -0.47 & & \\
\hline HIP 80616A & 11.631 & 0.700 & 4255 & 4275 & $4265 \pm 10$ & sdK6.0 & $21.1463 \pm 0.0533$ & $8.257 \pm 0.025$ & $0.513 \pm 0.014$ & -0.47 & & \\
\hline HIP $80616 \mathrm{~B}$ & 11.631 & 0.700 & 4255 & 4275 & $4265 \pm 10$ & $\mathrm{sdK} 6.0$ & $21.1463 \pm 0.0533$ & $8.257 \pm 0.025$ & $0.513 \pm 0.014$ & -0.47 & & \\
\hline HIP 82169 & 10.707 & 0.731 & 4177 & 4231 & $4204 \pm 27$ & dK6.4 & $33.0174 \pm 0.0419$ & $8.301 \pm 0.023$ & $0.529 \pm 0.023$ & +0.01 & & \\
\hline HIP 82844 & 10.637 & 0.657 & 4364 & 4361 & $4363 \pm 2$ & dK5.4 & $24.6289 \pm 0.0199$ & $7.594 \pm 0.022$ & $0.646 \pm 0.012$ & +0.47 & & \\
\hline HIP 86509 & 10.28 & 0.670 & 4331 & 4309 & $4320 \pm 22$ & dK5.7 & $22.7802 \pm 0.0522$ & $7.068 \pm 0.025$ & $0.850 \pm 0.030$ & & 7 & Binary? \\
\hline HIP 89320 & 10.93 & 0.731 & 4177 & - & $4177 \pm 41$ & dK6.4 & $22.7691 \pm 0.0204$ & $7.717 \pm 0.022$ & $0.709 \pm 0.040$ & & & Binary? \\
\hline HIP 91906 & 10.547 & 0.682 & 4300 & - & $4300 \pm 41$ & dK5.8 & $25.0406 \pm 0.0192$ & $7.540 \pm 0.022$ & $0.694 \pm 0.034$ & & & \\
\hline HIP 95223 & 9.93 & 0.645 & 4394 & 4392 & $4393 \pm 2$ & dK5.3 & $35.0926 \pm 0.0546$ & $7.656 \pm 0.023$ & $0.614 \pm 0.011$ & +0.13 & & \\
\hline HIP 97337 & 11.14 & 0.731 & 4177 & - & $4177 \pm 41$ & dK6.6 & $26.0538 \pm 0.0406$ & $8.219 \pm 0.023$ & $0.563 \pm 0.032$ & -0.01 & & \\
\hline HIP 99096 & 11.19 & 0.672 & 4326 & - & $4326 \pm 41$ & dK5.7 & $20.7713 \pm 0.0454$ & $7.777 \pm 0.025$ & $0.610 \pm 0.031$ & +0.12 & & \\
\hline HIP 102320 & 9.935 & 0.682 & 4300 & 4401 & $4350 \pm 51$ & dK5.5 & $24.0617 \pm 0.0268$ & $6.842 \pm 0.022$ & $0.922 \pm 0.053$ & & & Binary? \\
\hline HIP 103150 & 11.18 & 0.812 & 3985 & 3900 & $3942 \pm 42$ & $\mathrm{dK} 7.7$ & $30.9218 \pm 0.0675$ & $8.631 \pm 0.025$ & $0.581 \pm 0.041$ & -0.20 & & \\
\hline HIP 104078 & 11.61 & 0.746 & 4139 & - & $4139 \pm 41$ & $\mathrm{dK} 6.8$ & $19.909 \pm 0.562$ & $8.105 \pm 0.080$ & $0.613 \pm 0.052$ & & & \\
\hline HIP 105504 & 10.33 & 0.731 & 4177 & 4154 & $4166 \pm 12$ & dK6.6 & $24.0023 \pm 0.0726$ & $7.231 \pm 0.027$ & $0.896 \pm 0.028$ & & & Binary? \\
\hline HIP 106271A & 9.77 & 0.645 & 4394 & 4406 & $4400 \pm 6$ & dK5.2 & $23.0160 \pm 0.0651$ & $6.580 \pm 0.026$ & $1.002 \pm 0.023$ & & & PMS? \\
\hline HIP 106271B & 10.91 & 0.645 & 4394 & 4406 & $4400 \pm 6$ & $\mathrm{dK} 5.2$ & $22.4876 \pm 0.0336$ & $7.670 \pm 0.023$ & $0.607 \pm 0.013$ & & & \\
\hline HIP 106439 & 10.582 & 0.743 & 4146 & 4183 & $4165 \pm 18$ & sdK6.6 & $22.6957 \pm 0.0528$ & $7.362 \pm 0.025$ & $0.844 \pm 0.030$ & -1.46 & & Binary? \\
\hline HIP 109528 & 11.07 & 0.731 & 4177 & 4185 & $4181 \pm 4$ & sdK6.5 & $24.3926 \pm 0.1068$ & $8.006 \pm 0.029$ & $0.618 \pm 0.015$ & -1.18 & 2 & \\
\hline HIP 110245 & 10.17 & 0.728 & 4184 & 4422 & $4303 \pm 119$ & dK5.8 & $29.1588 \pm 0.0490$ & $7.494 \pm 0.024$ & $0.708 \pm 0.082$ & +0.04 & 4 & \\
\hline HIP 110401 & 10.675 & 0.737 & 4162 & 4230 & $4196 \pm 34$ & $\mathrm{dK} 6.4$ & $20.9375 \pm 1.0215$ & $7.280 \pm 0.123$ & $0.853 \pm 0.085$ & +0.09 & & Binary? \\
\hline HIP 110714 & 10.52 & 0.770 & 4078 & 4157 & $4117 \pm 40$ & dK6.9 & $30.4301 \pm 0.0416$ & $7.937 \pm 0.023$ & $0.676 \pm 0.038$ & +0.37 & 3 & \\
\hline HIP 111520 & 10.26 & 0.657 & 4364 & 4318 & $4341 \pm 23$ & dK5.6 & $19.4299 \pm 0.9547$ & $6.702 \pm 0.124$ & $0.990 \pm 0.084$ & +0.50 & & \\
\hline HIP 111657 & 10.571 & 0.701 & 4253 & 4387 & $4320 \pm 67$ & dK5.7 & $26.2023 \pm 0.1038$ & $7.663 \pm 0.029$ & $0.646 \pm 0.048$ & -0.29 & & \\
\hline HIP $112354 \mathrm{~A}$ & 10.23 & 0.657 & 4364 & - & $4364 \pm 41$ & dK5.4 & $23.0586 \pm 0.0404$ & $7.044 \pm 0.024$ & $0.831 \pm 0.041$ & & & Binary? \\
\hline HIP 112354B & 11.26 & 0.657 & 4364 & - & $4364 \pm 41$ & dK5.4 & $23.0108 \pm 0.0409$ & $8.070 \pm 0.024$ & $0.518 \pm 0.026$ & & & \\
\hline HIP 112389 & 10.664 & 0.686 & 4290 & - & $4290 \pm 41$ & dK5.9 & $24.9588 \pm 0.0876$ & $7.650 \pm 0.028$ & $0.665 \pm 0.035$ & & & \\
\hline HIP 113128 & 10.42 & 0.645 & 4394 & 4451 & $4422 \pm 29$ & dK5.1 & $14.8717 \pm 0.1969$ & $6.282 \pm 0.049$ & $1.131 \pm 0.058$ & +0.01 & 2 & Binary? \\
\hline HIP 113597A & 10.10 & 0.756 & 4114 & 4257 & $4185 \pm 72$ & dK6.5 & $31.3880 \pm 0.0485$ & $7.584 \pm 0.023$ & $0.749 \pm 0.065$ & & 2 & \\
\hline HIP 113597B & 10.66 & 0.756 & 4114 & 4257 & $4185 \pm 72$ & dK6.5 & $31.4730 \pm 0.0610$ & $8.150 \pm 0.024$ & $0.577 \pm 0.050$ & & 2 & \\
\hline HIP 115213 & 11.640 & 0.657 & 4364 & 4222 & $4293 \pm 71$ & dK5.9 & $15.9767 \pm 0.0478$ & $7.657 \pm 0.026$ & $0.661 \pm 0.051$ & & & \\
\hline HIP 116011 & 11.80 & 0.704 & 4245 & - & $4245 \pm 41$ & dK6.2 & $16.3781 \pm 0.0356$ & $7.871 \pm 0.025$ & $0.622 \pm 0.035$ & & 8 & \\
\hline HIP 116744 & 11.59 & 0.756 & 4114 & 4057 & $4085 \pm 28$ & $\mathrm{dK} 7.1$ & $19.6607 \pm 0.0786$ & $8.058 \pm 0.029$ & $0.658 \pm 0.032$ & & & \\
\hline HIP 117795 & 10.545 & 0.657 & 4364 & 4360 & $4362 \pm 2$ & dK5.4 & $37.3452 \pm 0.0319$ & $8.406 \pm 0.022$ & $0.445 \pm 0.008$ & & & Subdwarf? \\
\hline HIP $117809 \mathrm{~A}$ & 11.117 & 0.792 & 4023 & 3988 & $4006 \pm 17$ & $\mathrm{dK} 7.4$ & $26.7869 \pm 0.0405$ & $8.257 \pm 0.023$ & $0.644 \pm 0.023$ & +0.05 & & \\
\hline HIP 120148 & 10.574 & 0.731 & 4177 & 3959 & $4068 \pm 109$ & $\mathrm{dK} 7.2$ & $33.3417 \pm 0.0345$ & $8.189 \pm 0.022$ & $0.629 \pm 0.079$ & & & \\
\hline LHS 104 & 13.78 & 0.775 & 4066 & 3970 & $4018 \pm 48$ & sdK7.4 & $17.8248 \pm 0.0278$ & $10.035 \pm 0.023$ & $0.281 \pm 0.018$ & -1.33 & & \\
\hline LHS 3060 & 10.10 & 0.706 & 4240 & - & $4240 \pm 41$ & $\mathrm{dK} 6.2$ & $39.7700 \pm 0.0466$ & $8.098 \pm 0.023$ & $0.563 \pm 0.031$ & -0.05 & 1 & \\
\hline LHS 3613 & 10.97 & 0.758 & 4109 & 4328 & $4219 \pm 109$ & sdK6.3 & $32.6969 \pm 0.0372$ & $8.543 \pm 0.022$ & $0.467 \pm 0.057$ & +0.27 & & Subdwarf? \\
\hline STKM 1-178 & 11.23 & 0.694 & 4270 & - & $4270 \pm 41$ & $\mathrm{dK} 6.0$ & $22.0115 \pm 0.0797$ & $7.943 \pm 0.028$ & $0.590 \pm 0.031$ & & & \\
\hline STKM 1-188 & 11.15 & 0.768 & 4083 & - & $4083 \pm 41$ & $\mathrm{dK} 7.1$ & $20.4705 \pm 0.1121$ & $7.706 \pm 0.032$ & $0.775 \pm 0.048$ & +0.67 & & Binary? \\
\hline STKM 1-279 & 10.805 & 0.657 & 4364 & 4204 & $4284 \pm 80$ & dK5.9 & $22.8537 \pm 0.0397$ & $7.600 \pm 0.024$ & $0.684 \pm 0.057$ & & & \\
\hline STKM $1-420$ & 11.14 & 0.719 & 4207 & 4471 & $4339 \pm 132$ & sdK5.6 & $22.4898 \pm 0.0604$ & $7.900 \pm 0.026$ & $0.571 \pm 0.073$ & -0.60 & & \\
\hline STKM 1-450 & 10.25 & 0.731 & 4177 & 4237 & $4207 \pm 30$ & dK6.4 & $24.8205 \pm 0.0370$ & $7.224 \pm 0.023$ & $0.867 \pm 0.040$ & & & Binary? \\
\hline STKM 1-451 & 11.052 & 0.706 & 4240 & 4246 & $4243 \pm 3$ & $\mathrm{dK} 6.3$ & $15.6410 \pm 1.1599$ & $7.023 \pm 0.175$ & $0.921 \pm 0.087$ & +0.63 & & Binary? \\
\hline STKM 1-459 & 11.275 & 0.731 & 4177 & - & $4177 \pm 41$ & dK6.6 & $21.5318 \pm 0.0694$ & $7.940 \pm 0.027$ & $0.640 \pm 0.037$ & -0.15 & 1 & \\
\hline STKM $1-477$ & 10.72 & 0.700 & 4255 & 4412 & $4334 \pm 79$ & dK5.6 & $22.4472 \pm 0.0916$ & $7.476 \pm 0.029$ & $0.697 \pm 0.058$ & +0.27 & & \\
\hline STKM 1-478 & 11.55 & 0.756 & 4114 & 4034 & $4074 \pm 40$ & dK6.9 & $20.8276 \pm 0.0383$ & $8.143 \pm 0.024$ & $0.639 \pm 0.037$ & & 1 & \\
\hline STKM 1-498 & 11.51 & 0.768 & 4083 & 4274 & $4179 \pm 95$ & $\mathrm{dK} 6.5$ & $20.6941 \pm 0.0402$ & $8.089 \pm 0.024$ & $0.596 \pm 0.065$ & & & \\
\hline STKM $1-589 \mathrm{~A}$ & 11.51 & 0.682 & 4300 & - & $4300 \pm 41$ & dK5.8 & $16.0050 \pm 0.0452$ & $7.531 \pm 0.026$ & $0.697 \pm 0.036$ & & & \\
\hline STKM 1-589B & 11.73 & 0.682 & 4300 & - & $4300 \pm 41$ & dK5.8 & $16.1011 \pm 0.0439$ & $7.764 \pm 0.026$ & $0.626 \pm 0.032$ & & & \\
\hline
\end{tabular}


Table 2:: continued.

\begin{tabular}{|c|c|c|c|c|c|c|c|c|c|c|c|c|}
\hline Star & $\begin{array}{c}\mathrm{V} \\
(\mathrm{mag})\end{array}$ & $\begin{array}{c}(\mathrm{R}-\mathrm{I})_{C} \\
(\mathrm{mag})\end{array}$ & $\begin{array}{c}T_{\text {eff }}{ }^{1} \\
(\mathrm{~K})\end{array}$ & $\begin{array}{c}T_{e f f}^{2} \\
(\mathrm{~K})\end{array}$ & $T_{(\mathrm{K})} T_{\text {eff }}^{ \pm} 3 \sigma^{3}$ & $\begin{array}{l}\text { Spect. } \\
\text { Type }\end{array}$ & $\begin{array}{c}\pi \\
(\mathrm{m} ")\end{array}$ & $\begin{array}{c}M_{V} \\
(\mathrm{mag})\end{array}$ & $\begin{array}{c}R_{\star} \\
\left(R_{\odot}\right)\end{array}$ & {$[\mathrm{M} / \mathrm{H}]$} & $\begin{array}{l}\text { Hi. Res. } \\
\text { Spectra }\end{array}$ & Comments \\
\hline STKM 1-607 & 9.56 & 0.756 & 4114 & 4226 & $4170 \pm 56$ & dK6.6 & - & - & - & & & \\
\hline STKM 1-782 & 11.595 & 0.694 & 4270 & 4096 & $4183 \pm 87$ & dK6.5 & $14.0946 \pm 0.1210$ & $7.340 \pm 0.039$ & $0.839 \pm 0.091$ & & & Binary? \\
\hline STKM 1-1031 & 11.10 & 0.706 & 4240 & - & $4240 \pm 41$ & $\mathrm{dK} 6.2$ & $21.9784 \pm 0.0290$ & $7.810 \pm 0.023$ & $0.643 \pm 0.036$ & & & \\
\hline STKM 1-1036 & 11.78 & 0.743 & 4146 & & $4146 \pm 41$ & dK6.7 & $16.0774 \pm 0.0282$ & $7.811 \pm 0.024$ & $0.698 \pm 0.040$ & -0.08 & & \\
\hline STKM 1-1131 & 11.969 & 0.731 & 4177 & 3988 & $4083 \pm 94$ & $\mathrm{dK} 7.1$ & $18.2349 \pm 0.0358$ & $8.274 \pm 0.024$ & $0.597 \pm 0.066$ & & & \\
\hline STKM 1-1171A & 12.34 & 0.731 & 4177 & - & $4177 \pm 41$ & $\mathrm{dK} 6.6$ & $10.6471 \pm 0.0684$ & $7.476 \pm 0.076$ & $0.792 \pm 0.049$ & & & Binary? \\
\hline STKM 1-1197 & 11.99 & 0.706 & 4240 & - & $4240 \pm 41$ & dK6.2 & $14.3038 \pm 0.0239$ & $7.767 \pm 0.024$ & $0.656 \pm 0.037$ & & & \\
\hline STKM 1-1211 & 11.545 & 0.682 & 4300 & 4162 & $4231 \pm 69$ & dK5.6 & $12.4548 \pm 0.1037$ & $7.022 \pm 0.038$ & $0.931 \pm 0.084$ & & & Binary? \\
\hline STKM 1-1241 & 10.51 & 0.657 & 4364 & 4364 & $4364 \pm 1$ & dK5.4 & $21.7685 \pm 0.3568$ & $7.199 \pm 0.055$ & $0.774 \pm 0.026$ & +0.11 & & \\
\hline STKM 1-1362 & 11.29 & 0.645 & 4394 & 4277 & $4336 \pm 58$ & dK5.6 & $13.7921 \pm 0.2949$ & $6.988 \pm 0.066$ & $0.871 \pm 0.074$ & -0.41 & & Binary? \\
\hline STKM 1-1384 & 11.035 & 0.682 & 4300 & 4248 & $4274 \pm 26$ & dK6.0 & $21.5733 \pm 0.0461$ & $7.705 \pm 0.025$ & $0.657 \pm 0.026$ & & & \\
\hline STKM 1-1392 & 11.025 & 0.694 & 4270 & 4229 & $4250 \pm 21$ & $\mathrm{dK} 6.1$ & $21.7708 \pm 0.0296$ & $7.714 \pm 0.023$ & $0.666 \pm 0.024$ & & & \\
\hline STKM 1-1446 & 10.99 & 0.768 & 4083 & 4241 & $4162 \pm 79$ & dK6.6 & $13.7189 \pm 0.2221$ & $6.677 \pm 0.055$ & $1.160 \pm 0.127$ & +0.56 & & PMS? \\
\hline STKM 1-1486 & 11.490 & 0.657 & 4364 & 4203 & $4284 \pm 81$ & dK5.9 & $17.8802 \pm 0.0359$ & $7.752 \pm 0.024$ & $0.638 \pm 0.053$ & & & \\
\hline STKM 1-1618 & 10.67 & 0.731 & 4177 & 4098 & $4138 \pm 39$ & $\mathrm{dK} 6.8$ & $24.7461 \pm 0.0192$ & $7.638 \pm 0.022$ & $0.761 \pm 0.041$ & & & \\
\hline STKM 1-1651 & 10.96 & 0.700 & 4255 & - & $4255 \pm 41$ & dK6.1 & $17.4699 \pm 0.0239$ & $7.171 \pm 0.023$ & $0.852 \pm 0.043$ & & & Binary? \\
\hline STKM 1-1669 & 11.03 & 0.682 & 4300 & 4100 & $4200 \pm 100$ & dK6.4 & $21.8654 \pm 0.0416$ & $7.729 \pm 0.024$ & $0.691 \pm 0.079$ & & & \\
\hline STKM 1-1745 & 10.893 & 0.682 & 4300 & 4338 & $4319 \pm 19$ & dK5.7 & $21.8801 \pm 0.0749$ & $7.593 \pm 0.027$ & $0.668 \pm 0.023$ & +0.09 & & \\
\hline STKM 1-1792 & 11.355 & 0.719 & 4207 & 4347 & $4277 \pm 70$ & $\mathrm{dK} 6.0$ & $21.5613 \pm 0.4031$ & $8.023 \pm 0.060$ & $0.566 \pm 0.052$ & & & \\
\hline STKM 1-2011 & 11.372 & 0.694 & 4270 & - & $4270 \pm 41$ & dK6.0 & $17.9886 \pm 0.0484$ & $7.647 \pm 0.026$ & $0.676 \pm 0.035$ & +0.43 & & \\
\hline STKM 1-2095 & 11.035 & 0.657 & 4364 & 4220 & $4292 \pm 72$ & dK5.9 & $14.7684 \pm 0.1780$ & $6.882 \pm 0.046$ & $0.946 \pm 0.082$ & & & Binary? \\
\hline STKM 1-2137 & 11.715 & 0.768 & 4083 & 4393 & $4238 \pm 155$ & $\mathrm{dK} 6.2$ & $19.1583 \pm 0.0540$ & $8.127 \pm 0.026$ & $0.556 \pm 0.095$ & & & \\
\hline MCC 66 & 10.40 & 0.694 & 4270 & 4409 & $4340 \pm 69$ & dK5.6 & $24.5442 \pm 0.0624$ & $7.350 \pm 0.026$ & $0.735 \pm 0.054$ & -0.34 & & Binary? \\
\hline MCC 189 & 10.547 & 0.682 & 4300 & 4387 & $4344 \pm 44$ & dK5.6 & $25.0406 \pm 0.0192$ & $7.540 \pm 0.022$ & $0.672 \pm 0.034$ & +0.28 & & \\
\hline MCC 374 & 11.380 & 0.719 & 4207 & 4100 & $4154 \pm 53$ & $\mathrm{dK} 6.7$ & $21.3328 \pm 0.0492$ & $8.025 \pm 0.025$ & $0.628 \pm 0.043$ & +0.30 & & \\
\hline L $477-10$ & 10.82 & 0.719 & 4207 & - & $4207 \pm 41$ & dK6.4 & $29.9181 \pm 0.0224$ & $8.200 \pm 0.022$ & $0.553 \pm 0.031$ & -0.09 & & \\
\hline LTT 15917 & 10.71 & 0.781 & 4050 & 4099 & $4075 \pm 24$ & dK7.1 & $31.9352 \pm 0.0450$ & $8.231 \pm 0.023$ & $0.613 \pm 0.025$ & +0.00 & & \\
\hline \multirow[t]{2}{*}{$\mathrm{BD}+13$ 5059B } & 10.239 & 0.786 & 4038 & 4031 & $4034 \pm 4$ & $\mathrm{dK} 7.3$ & $39.6998 \pm 0.0472$ & $8.233 \pm 0.023$ & $0.635 \pm 0.015$ & & & \\
\hline & & & & & M0-M1 sampl & & & & & & & \\
\hline GJ 1170 & 11.269 & 1.059 & 3723 & 3702 & $3713 \pm 11$ & $\mathrm{dM} 1.3$ & $42.2090 \pm 0.0345$ & $9.396 \pm 0.022$ & $0.529 \pm 0.019$ & -0.16 & & \\
\hline GJ 2090 & 11.774 & 1.046 & 3735 & 3725 & $3730 \pm 5$ & $\mathrm{dM} 1.2$ & $36.9881 \pm 0.0485$ & $9.614 \pm 0.023$ & $0.469 \pm 0.014$ & +0.00 & & \\
\hline GJ 3108 & 10.402 & 0.954 & 3815 & 3785 & $3800 \pm 15$ & $\mathrm{dM} 0.7$ & $45.3802 \pm 0.0395$ & $8.686 \pm 0.022$ & $0.664 \pm 0.026$ & & & \\
\hline GJ $3186 \mathrm{~A}$ & 11.832 & 1.072 & 3711 & - & $3711 \pm 30$ & $\mathrm{dM} 1.4$ & $42.6652 \pm 0.0407$ & $9.982 \pm 0.022$ & $0.404 \pm 0.024$ & & & Subdwarf? \\
\hline GJ $3186 B$ & 11.832 & 1.072 & 3711 & - & $3711 \pm 30$ & dM1.4 & $42.6652 \pm 0.0407$ & $9.982 \pm 0.022$ & $0.404 \pm 0.024$ & & & Subdwarf? \\
\hline GJ 3212 & 11.619 & 1.046 & 3735 & 4075 & $3905 \pm 170$ & $\operatorname{sdK} 7.9$ & $45.1245 \pm 0.0495$ & $9.891 \pm 0.022$ & $0.339 \pm 0.081$ & -0.07 & & Subdwarf? \\
\hline GJ 3251 & 11.401 & 1.084 & 3700 & 3693 & $3697 \pm 4$ & $\mathrm{dM} 1.5$ & $25.2910 \pm 0.0362$ & $8.416 \pm 0.023$ & $0.846 \pm 0.024$ & & 1 & Binary? \\
\hline GJ 3260 & 11.431 & 1.059 & 3723 & 3715 & $3719 \pm 4$ & $\mathrm{dM} 1.3$ & $29.5902 \pm 0.0353$ & $8.787 \pm 0.023$ & $0.695 \pm 0.019$ & -0.09 & & Binary? \\
\hline GJ 3305 & 10.59 & 1.097 & 3688 & 3698 & $3693 \pm 5$ & $\mathrm{dM} 1.5$ & $37.9633 \pm 0.3662$ & $8.487 \pm 0.041$ & $0.822 \pm 0.031$ & -0.01 & 6 & Binary? \\
\hline GJ 3371 & 10.253 & 1.059 & 3723 & 3712 & $3717 \pm 5$ & dM1.3 & $74.1524 \pm 0.0432$ & $9.604 \pm 0.021$ & $0.478 \pm 0.014$ & +0.07 & 1 & \\
\hline
\end{tabular}


Table 2:: continued.

\begin{tabular}{|c|c|c|c|c|c|c|c|c|c|c|c|c|}
\hline Star & $\underset{(\mathrm{mag})}{\mathrm{V}}$ & $\begin{array}{c}(\mathrm{R}-\mathrm{I})_{C} \\
(\mathrm{mag})\end{array}$ & $\begin{array}{c}T_{e f f}^{1} \\
(\mathrm{~K})\end{array}$ & $\begin{array}{c}T_{e f f}^{2} \\
(\mathrm{~K})\end{array}$ & $\begin{array}{c}T_{e f f} \pm 3 \sigma^{3} \\
(\mathrm{~K})\end{array}$ & $\begin{array}{l}\text { Spect. } \\
\text { Type }\end{array}$ & $\begin{array}{c}\pi \\
(\mathrm{m} ")\end{array}$ & $\begin{array}{c}M_{V} \\
(\mathrm{mag})\end{array}$ & $\begin{array}{c}R_{\star} \\
\left(R_{\odot}\right)\end{array}$ & {$[\mathrm{M} / \mathrm{H}]$} & $\begin{array}{l}\text { Hi. Res. } \\
\text { Spectra }\end{array}$ & Comments \\
\hline GJ 3408B & 12.160 & 1.062 & 3720 & 3705 & $3712 \pm 8$ & $\operatorname{sdM} 1.3$ & $38.7792 \pm 0.0414$ & $10.103 \pm 0.022$ & $0.382 \pm 0.012$ & & & Subdwarf? \\
\hline GJ $3409 B$ & 12.121 & 1.034 & 3746 & 3753 & $3750 \pm 3$ & sdM1.1 & $38.7792 \pm 0.0414$ & $10.064 \pm 0.022$ & $0.373 \pm 0.010$ & -0.33 & 1 & Subdwarf? \\
\hline GJ 3432 & 11.279 & 1.059 & 3723 & 3771 & $3747 \pm 24$ & $\mathrm{dM} 1.1$ & $39.6119 \pm 0.0439$ & $9.268 \pm 0.022$ & $0.539 \pm 0.028$ & -0.41 & & \\
\hline GJ $3501 \mathrm{~A}$ & 11.185 & 1.072 & 3711 & 3728 & $3719 \pm 9$ & $\mathrm{dM} 1.3$ & $39.9747 \pm 0.1231$ & $9.194 \pm 0.027$ & $0.576 \pm 0.021$ & -0.04 & 14 & \\
\hline GJ 3759 & 10.935 & 1.082 & 3702 & 3740 & $3721 \pm 2$ & sdM1.3 & $59.3162 \pm 0.0403$ & $9.801 \pm 0.021$ & $0.435 \pm 0.011$ & -1.05 & 14 & \\
\hline GJ 3765 & 11.020 & 1.046 & 3735 & 3698 & $3717 \pm 19$ & $\mathrm{dM} 1.3$ & $56.1079 \pm 0.1467$ & $9.765 \pm 0.026$ & $0.444 \pm 0.021$ & -0.10 & & \\
\hline GJ 3874 & 11.271 & 1.033 & 3746 & 3655 & $3701 \pm 46$ & $\mathrm{dM} 1.4$ & $40.2330 \pm 0.1053$ & $9.294 \pm 0.026$ & $0.562 \pm 0.046$ & -0.45 & & \\
\hline GJ 3997 & 10.365 & 1.034 & 3746 & 3705 & $3726 \pm 21$ & $\mathrm{dM} 1.2$ & $76.5790 \pm 0.2030$ & $9.786 \pm 0.026$ & $0.435 \pm 0.022$ & -0.25 & 1 & Subdwarf? \\
\hline GJ 4041 & 10.96 & 1.046 & 3735 & - & $3735 \pm 22$ & $\mathrm{dM} 1.2$ & $46.2650 \pm 0.0220$ & $9.286 \pm 0.021$ & $0.542 \pm 0.026$ & & 1 & \\
\hline GJ 4153A & 12.25 & 1.059 & 3723 & 3709 & $3716 \pm 7$ & $\mathrm{sdM} 1.3$ & $23.6590 \pm 0.0524$ & $9.120 \pm 0.025$ & $0.598 \pm 0.019$ & -0.89 & 1 & \\
\hline GJ 4153B & 12.25 & 1.059 & 3723 & 3709 & $3716 \pm 7$ & $\operatorname{sdM} 1.3$ & $23.6590 \pm 0.0524$ & $9.120 \pm 0.025$ & $0.598 \pm 0.019$ & -0.89 & 1 & \\
\hline GJ $4153 \mathrm{C}$ & 12.25 & 1.059 & 3723 & 3709 & $3716 \pm 7$ & $\mathrm{sdM} 1.3$ & $23.6590 \pm 0.0524$ & $9.120 \pm 0.025$ & $0.598 \pm 0.019$ & -0.89 & 1 & \\
\hline GJ $4160 \mathrm{~A}$ & 11.25 & 1.072 & 3711 & 3701 & $3706 \pm 5$ & $\mathrm{dM} 1.4$ & $40.4913 \pm 0.0445$ & $9.287 \pm 0.022$ & $0.560 \pm 0.016$ & & 2 & \\
\hline GJ 4195 & 11.622 & 1.084 & 3700 & 3678 & $3689 \pm 11$ & $\mathrm{dM} 1.5$ & $38.7994 \pm 0.0649$ & $9.566 \pm 0.024$ & $0.503 \pm 0.019$ & -0.44 & 1 & \\
\hline GJ 4306 & 10.522 & 1.046 & 3735 & 3722 & $3729 \pm 7$ & $\mathrm{dM} 1.2$ & $59.0165 \pm 0.0581$ & $9.377 \pm 0.022$ & $0.524 \pm 0.016$ & -0.07 & 3 & \\
\hline GJ 4346 & 11.21 & 1.034 & 3746 & - & $3746 \pm 22$ & $\mathrm{dM} 1.1$ & $35.2475 \pm 0.0430$ & $8.946 \pm 0.023$ & $0.626 \pm 0.031$ & & 1 & \\
\hline GJ 4359A & 12.16 & 1.072 & 3711 & - & $3711 \pm 22$ & sdM1.3 & $34.3350 \pm 0.0220$ & $9.839 \pm 0.021$ & $0.432 \pm 0.021$ & & 1 & Subdwarf? \\
\hline GJ 4359B & 12.16 & 1.072 & 3711 & - & $3711 \pm 22$ & $\operatorname{sdM} 1.3$ & $34.3350 \pm 0.0220$ & $9.839 \pm 0.021$ & $0.432 \pm 0.021$ & & 1 & Subdwarf? \\
\hline GJ 9397 & 11.963 & 1.063 & 3719 & 3713 & $3716 \pm 3$ & $\mathrm{sdM} 1.3$ & $33.3099 \pm 0.0412$ & $9.576 \pm 0.023$ & $0.485 \pm 0.013$ & -0.54 & & \\
\hline Gl 17.1 & 10.394 & 0.970 & 3802 & 3820 & $3811 \pm 9$ & $\mathrm{dM} 0.6$ & $56.8562 \pm 0.0315$ & $9.168 \pm 0.021$ & $0.525 \pm 0.017$ & & & \\
\hline Gl 21 & 10.539 & 1.034 & 3746 & 3710 & $3728 \pm 18$ & $\mathrm{sdM} 1.2$ & $61.8444 \pm 0.0250$ & $9.496 \pm 0.021$ & $0.496 \pm 0.022$ & -1.03 & 9 & \\
\hline Gl 56.3B & 10.715 & 0.858 & 3923 & 3934 & $3928 \pm 6$ & $\mathrm{dK} 7.8$ & $36.4322 \pm 0.3670$ & $8.522 \pm 0.042$ & $0.621 \pm 0.023$ & & & \\
\hline Gl 63 & 11.22 & 1.10 & 3685 & 3716 & $3700 \pm 16$ & sdM1.4 & $76.1309 \pm 0.0565$ & $10.628 \pm 0.022$ & $0.304 \pm 0.013$ & & 7 & Subdwarf? \\
\hline Gl 79 & 8.906 & 0.932 & 3833 & 3842 & $3837 \pm 5$ & $\mathrm{dM} 0.4$ & $90.4253 \pm 0.0480$ & $8.687 \pm 0.021$ & $0.637 \pm 0.017$ & & & \\
\hline Gl 96 & 9.413 & 1.019 & 3759 & 3735 & $3747 \pm 12$ & $\mathrm{dM} 1.1$ & $83.7829 \pm 0.0650$ & $9.029 \pm 0.022$ & $0.602 \pm 0.022$ & +0.14 & 55 & \\
\hline Gl 118.2B & 10.02 & - & - & 3961 & $3961 \pm$ & $\mathrm{dK} 7.6$ & $44.3828 \pm 0.0672$ & $8.256 \pm 0.023$ & $0.677 \pm 0.031$ & & & \\
\hline Gl 126 & 11.589 & 1.030 & 3749 & 3830 & $3789 \pm 51$ & $\mathrm{dM} 0.7$ & $39.8098 \pm 0.0259$ & $9.589 \pm 0.021$ & $0.444 \pm 0.037$ & +0.09 & & Subdwarf? \\
\hline Gl 155.1 & 11.067 & 1.039 & 3741 & 3690 & $3716 \pm 26$ & $\mathrm{dM} 1.3$ & $56.9576 \pm 0.0453$ & $9.845 \pm 0.022$ & $0.428 \pm 0.023$ & +0.05 & 9 & Subdwarf? \\
\hline Gl 162 & 10.197 & 1.047 & 3734 & 3703 & $3718 \pm 15$ & $\mathrm{dM} 1.3$ & $71.8130 \pm 0.0431$ & $9.478 \pm 0.021$ & $0.506 \pm 0.020$ & +0.69 & 9 & \\
\hline Gl 169 & 8.318 & 0.831 & 3960 & 4096 & $4028 \pm 68$ & $\mathrm{dK} 7.3$ & $88.9622 \pm 0.0494$ & $8.064 \pm 0.021$ & $0.690 \pm 0.058$ & & & Binary? \\
\hline Gl 172 & 8.627 & 0.849 & 3935 & 3922 & $3928 \pm 7$ & $\mathrm{dK} 7.8$ & $100.7797 \pm 0.0640$ & $8.644 \pm 0.021$ & $0.587 \pm 0.016$ & & & \\
\hline Gl 205 & 7.967 & 1.088 & 3696 & 3712 & $3704 \pm 8$ & $\mathrm{dM} 1.5$ & $175.4287 \pm 0.0672$ & $9.188 \pm 0.021$ & $0.588 \pm 0.019$ & +0.13 & 58 & \\
\hline Gl 208 & 8.882 & 0.875 & 3901 & 3973 & $3937 \pm 36$ & $\mathrm{dK} 7.7$ & $87.4367 \pm 0.0562$ & $8.590 \pm 0.021$ & $0.596 \pm 0.037$ & & & \\
\hline Gl 212 & 9.779 & 1.044 & 3736 & 3736 & $3736 \pm 1$ & $\mathrm{dM} 1.2$ & $81.4300 \pm 0.0487$ & $9.333 \pm 0.021$ & $0.530 \pm 0.012$ & +0.21 & 1 & \\
\hline Gl $229 \mathrm{~A}$ & 8.125 & 1.102 & 3683 & 3745 & $3714 \pm 31$ & dM1.3 & $173.6955 \pm 0.0457$ & $9.324 \pm 0.021$ & $0.546 \pm 0.033$ & -0.03 & 2 & \\
\hline Gl 239 & 9.602 & 0.943 & 3824 & 3747 & $3785 \pm 38$ & $\mathrm{dM} 0.8$ & $99.9164 \pm 0.0503$ & $9.600 \pm 0.021$ & $0.443 \pm 0.030$ & & & Subdwarf? \\
\hline Gl 272 & 10.544 & 1.081 & 3703 & 3715 & $3709 \pm 12$ & $\mathrm{dM} 1.3$ & $58.9849 \pm 0.0420$ & $9.398 \pm 0.022$ & $0.531 \pm 0.020$ & & & \\
\hline Gl 275.1 & 10.832 & 1.059 & 3723 & 3758 & $3741 \pm 19$ & $\mathrm{dM} 1.2$ & $42.7515 \pm 0.0404$ & $8.987 \pm 0.022$ & $0.618 \pm 0.028$ & -0.30 & & \\
\hline Gl 277.1 & 10.397 & 1.036 & 3744 & 3793 & $3768 \pm 25$ & sdM0.9 & $84.6499 \pm 0.0356$ & $10.035 \pm 0.021$ & $0.370 \pm 0.019$ & & & Subdwarf? \\
\hline Gl 281 & 9.614 & 0.923 & 3836 & 3797 & $3816 \pm 19$ & $\mathrm{dM} 0.5$ & $66.4106 \pm 0.0543$ & $8.725 \pm 0.022$ & $0.641 \pm 0.028$ & & & \\
\hline Gl $301 \mathrm{~A}$ & 9.377 & 1.084 & 3700 & 3955 & $3828 \pm 128$ & $\mathrm{dM} 0.4$ & $48.0282 \pm 1.4182$ & $7.784 \pm 0.083$ & $0.975 \pm 0.210$ & +0.24 & & Binary? \\
\hline Gl 338A & 7.645 & 0.860 & 3921 & 3896 & $3908 \pm 12$ & $\mathrm{dK} 7.9$ & $157.8796 \pm 0.0366$ & $8.637 \pm 0.021$ & $0.602 \pm 0.020$ & & & \\
\hline Gl 338B & 7.72 & 0.860 & 3921 & 3840 & $3880 \pm 40$ & $\mathrm{dM} 0.0$ & $157.8851 \pm 0.0414$ & $8.712 \pm 0.021$ & $0.600 \pm 0.040$ & & & \\
\hline Gl 353 & 10.221 & 1.014 & 3764 & 3845 & $3804 \pm 41$ & $\mathrm{dM} 0.6$ & $73.6683 \pm 0.0483$ & $9.557 \pm 0.021$ & $0.443 \pm 0.031$ & & & Subdwarf? \\
\hline Gl 369 & 10.038 & 1.020 & 3758 & 3742 & $3750 \pm 8$ & $\mathrm{dM} 1.1$ & $76.2323 \pm 0.0475$ & $9.449 \pm 0.021$ & $0.495 \pm 0.016$ & -0.20 & & \\
\hline Gl 429.2 & 12.40 & 1.054 & 3727 & & $3727 \pm 22$ & $\operatorname{sdM} 1.2$ & $36.9232 \pm 0.0510$ & $10.236 \pm 0.023$ & $0.353 \pm 0.018$ & & 30 & Subdwarf? \\
\hline Gl 430.1 & 10.269 & 1.038 & 3732 & 3760 & $3746 \pm 21$ & $\mathrm{dM} 1.1$ & $63.1910 \pm 0.0507$ & $9.272 \pm 0.022$ & $0.539 \pm 0.026$ & +0.53 & 4 & \\
\hline Gl 438 & 10.35 & 1.085 & 3699 & 3671 & $3685 \pm 14$ & sdM1.5 & $90.7992 \pm 0.0353$ & $10.140 \pm 0.021$ & $0.388 \pm 0.015$ & -0.05 & 21 & Subdwarf? \\
\hline Gl 456 & 11.271 & 0.938 & 3828 & 3886 & $3857 \pm 29$ & $\mathrm{dM} 0.2$ & $34.0638 \pm 0.0827$ & $8.932 \pm 0.025$ & $0.556 \pm 0.032$ & -0.31 & & \\
\hline Gl 458.2 & 10.546 & 0.939 & 3828 & 3844 & $3836 \pm 8$ & $\mathrm{dM} 0.4$ & $44.5245 \pm 0.0789$ & $8.789 \pm 0.024$ & $0.608 \pm 0.019$ & & & \\
\hline Gl 459.3 & 10.643 & 1.005 & 3772 & 3806 & $3789 \pm 17$ & $\mathrm{dM} 0.8$ & $41.8403 \pm 0.0441$ & $8.751 \pm 0.022$ & $0.653 \pm 0.027$ & & & \\
\hline Gl 471 & 9.684 & 0.936 & 3830 & 3817 & $3823 \pm 7$ & $\mathrm{dM} 0.5$ & $73.7640 \pm 0.4146$ & $9.023 \pm 0.032$ & $0.554 \pm 0.019$ & & & \\
\hline Gl 488 & 8.482 & 0.829 & 3962 & 3891 & $3926 \pm 35$ & $\mathrm{dK} 7.8$ & $93.8820 \pm 0.0508$ & $8.345 \pm 0.021$ & $0.675 \pm 0.041$ & & & \\
\hline
\end{tabular}


Table 2:: continued.

\begin{tabular}{|c|c|c|c|c|c|c|c|c|c|c|c|c|}
\hline Star & $\underset{(\mathrm{mag})}{\mathrm{V}}$ & $\begin{array}{c}(\mathrm{R}-\mathrm{I})_{C} \\
(\mathrm{mag})\end{array}$ & $\begin{array}{c}T_{\text {eff }}{ }^{1} \\
(\mathrm{~K})\end{array}$ & $\begin{array}{c}T_{\text {eff }}^{2} \\
(\mathrm{~K})\end{array}$ & $\begin{array}{c}T_{\text {eff }} \pm 3 \sigma^{3} \\
(\mathrm{~K})\end{array}$ & $\begin{array}{l}\text { Spect. } \\
\text { Type }\end{array}$ & $\begin{array}{c}\pi \\
(\mathrm{m} ")\end{array}$ & $\begin{array}{c}M_{V} \\
\text { (mag) }\end{array}$ & $\begin{array}{c}R_{\star} \\
\left(R_{\odot}\right)\end{array}$ & {$[\mathrm{M} / \mathrm{H}]$} & $\begin{array}{l}\text { Hi. Res. } \\
\text { Spectra }\end{array}$ & Comments \\
\hline Gl 505B & 9.522 & 1.018 & 3760 & 3840 & $3800 \pm 40$ & $\mathrm{dM} 0.7$ & $91.1065 \pm 0.0461$ & $9.320 \pm 0.021$ & $0.496 \pm 0.034$ & & & \\
\hline Gl 507A & 9.570 & 1.037 & 3743 & 3652 & $3698 \pm 45$ & dM1.4 & $74.6102 \pm 0.2946$ & $8.934 \pm 0.029$ & $0.665 \pm 0.054$ & -0.11 & & \\
\hline Gl 514 & 9.027 & 1.063 & 3719 & 3766 & $3743 \pm 23$ & dM1.1 & $131.2428 \pm 0.0543$ & $9.617 \pm 0.021$ & $0.461 \pm 0.023$ & -0.10 & 171 & \\
\hline Gl 521 & 10.215 & 1.083 & 3701 & 3694 & $3697 \pm 4$ & $\mathrm{dM} 1.5$ & $74.8051 \pm 0.0257$ & $9.585 \pm 0.021$ & $0.494 \pm 0.013$ & -0.16 & 36 & \\
\hline Gl 525 & 9.780 & 0.876 & 3899 & 3860 & $3879 \pm 19$ & $\mathrm{dM} 0.0$ & $77.4471 \pm 0.0353$ & $9.225 \pm 0.021$ & $0.474 \pm 0.020$ & & & \\
\hline Gl 536 & 9.714 & 1.088 & 3696 & 3747 & $3722 \pm 25$ & $\mathrm{dM} 1.3$ & $96.0398 \pm 0.0872$ & $9.626 \pm 0.022$ & $0.471 \pm 0.025$ & -0.14 & 177 & \\
\hline Gl 540 & 10.350 & 1.063 & 3719 & 3754 & $3737 \pm 17$ & $\operatorname{sdM1.2}$ & $58.1724 \pm 0.0302$ & $9.174 \pm 0.021$ & $0.570 \pm 0.024$ & -1.20 & 41 & \\
\hline Gl 606 & 10.492 & 1.078 & 3705 & 3704 & $3705 \pm 1$ & $\mathrm{dM} 1.4$ & $75.2306 \pm 0.0513$ & $9.874 \pm 0.021$ & $0.428 \pm 0.010$ & +0.18 & 41 & \\
\hline Gl 611.3 & 11.573 & 1.070 & 3713 & 3744 & $3729 \pm 15$ & $\mathrm{dM} 1.2$ & $28.5148 \pm 0.0629$ & $8.848 \pm 0.025$ & $0.668 \pm 0.028$ & -0.05 & & \\
\hline Gl 637 & 11.326 & 1.105 & 3681 & 3714 & $3697 \pm 17$ & $\mathrm{sdM} 1.5$ & $63.4448 \pm 0.0290$ & $10.338 \pm 0.021$ & $0.349 \pm 0.015$ & & 18 & Subdwarf? \\
\hline Gl 642 & 10.749 & 0.923 & 3836 & 3921 & $3878 \pm 43$ & $\mathrm{dM} 0.0$ & $49.7925 \pm 0.0384$ & $9.235 \pm 0.022$ & $0.473 \pm 0.034$ & & & \\
\hline Gl 649 & 9.662 & 1.078 & 3705 & 3678 & $3692 \pm 14$ & $\mathrm{dM} 1.5$ & $96.3141 \pm 0.0311$ & $9.580 \pm 0.021$ & $0.497 \pm 0.020$ & -0.14 & 4 & \\
\hline Gl 666B & 8.695 & 1.034 & 3746 & 3840 & $3793 \pm 47$ & $\mathrm{dM} 0.7$ & $120.1791 \pm 0.4767$ & $9.094 \pm 0.029$ & $0.555 \pm 0.045$ & +0.27 & & \\
\hline Gl $678.1 \mathrm{~A}$ & 9.334 & 0.978 & 3795 & 3741 & $3768 \pm 27$ & $\mathrm{dM} 0.9$ & $98.8491 \pm 0.0403$ & $9.309 \pm 0.021$ & $0.517 \pm 0.028$ & & & \\
\hline Gl 685 & 9.986 & 1.096 & 3689 & 3780 & $3734 \pm 46$ & $\mathrm{dM} 1.2$ & $68.8254 \pm 0.0392$ & $9.175 \pm 0.021$ & $0.571 \pm 0.045$ & & & \\
\hline Gl 709 & 10.296 & 0.992 & 3783 & 3767 & $3775 \pm 8$ & $\mathrm{dM} 0.9$ & $57.3252 \pm 0.0203$ & $9.088 \pm 0.021$ & $0.568 \pm 0.018$ & & & \\
\hline Gl 723 & 11.422 & 1.085 & 3699 & 3691 & $3695 \pm 4$ & sdM1.5 & $56.3274 \pm 0.0474$ & $10.176 \pm 0.022$ & $0.377 \pm 0.010$ & -0.45 & 10 & Subdwarf? \\
\hline Gl 740 & 9.225 & 1.020 & 3759 & 3754 & $3756 \pm 2$ & $\mathrm{dM} 1.0$ & $90.0477 \pm 0.0490$ & $8.997 \pm 0.021$ & $0.605 \pm 0.015$ & +0.00 & & \\
\hline Gl 747.4 & 11.32 & 0.797 & 4010 & 3924 & $3967 \pm 43$ & sdK7.6 & $42.4954 \pm 0.0336$ & $9.462 \pm 0.022$ & $0.386 \pm 0.027$ & & & Subdwarf? \\
\hline Gl 756 & 11.518 & 1.098 & 3687 & 3718 & $3703 \pm 15$ & dM1.4 & $42.4277 \pm 0.0303$ & $9.656 \pm 0.022$ & $0.474 \pm 0.019$ & -0.25 & 2 & \\
\hline Gl 767A & 9.998 & 1.127 & 3659 & 3766 & $3713 \pm 53$ & sdM1.3 & $71.7677 \pm 0.0559$ & $9.278 \pm 0.022$ & $0.558 \pm 0.049$ & -1.00 & 1 & \\
\hline Gl 809 & 8.577 & 1.063 & 3719 & 3714 & $3717 \pm 3$ & $\mathrm{dM} 1.3$ & $142.0341 \pm 0.0305$ & $9.339 \pm 0.020$ & $0.540 \pm 0.014$ & -0.22 & 3 & \\
\hline Gl 817 & 11.468 & 1.075 & 3708 & 3717 & $3713 \pm 5$ & $\mathrm{dM} 1.3$ & $54.6973 \pm 0.0445$ & $10.158 \pm 0.022$ & $0.372 \pm 0.011$ & -0.46 & 38 & \\
\hline Gl 835 & 9.924 & 1.034 & 3746 & 3746 & $3746 \pm 1$ & dM1.1 & $76.2244 \pm 0.0440$ & $9.334 \pm 0.021$ & $0.524 \pm 0.012$ & -0.17 & 1 & \\
\hline Gl 838.3B & 11.482 & 0.970 & 3802 & 3793 & $3797 \pm 4$ & $\mathrm{dM} 0.7$ & $33.7487 \pm 0.0378$ & $9.123 \pm 0.022$ & $0.545 \pm 0.015$ & & & \\
\hline Gl 846 & 9.163 & 0.978 & 3795 & 3778 & $3786 \pm 10$ & dM0.8 & $94.7419 \pm 0.1406$ & $9.046 \pm 0.023$ & $0.572 \pm 0.020$ & & & \\
\hline Gl 855 & 10.708 & 1.052 & 3729 & 3750 & $3739 \pm 11$ & $\mathrm{dM} 1.2$ & $52.6817 \pm 0.0413$ & $9.316 \pm 0.022$ & $0.532 \pm 0.019$ & & 41 & \\
\hline Gl 863 & 10.339 & 1.068 & 3714 & 3731 & $3722 \pm 10$ & $\mathrm{dM} 1.3$ & $77.0259 \pm 0.0562$ & $9.772 \pm 0.022$ & $0.440 \pm 0.015$ & & 15 & Subdwarf? \\
\hline Gl 864 & 9.961 & 0.980 & 3793 & 3774 & $3783 \pm 9$ & dM0.8 & $60.4036 \pm 0.0418$ & $8.866 \pm 0.022$ & $0.623 \pm 0.020$ & & & \\
\hline Gl 875 & 9.857 & 0.942 & 3825 & 3892 & $3858 \pm 34$ & $\mathrm{dM} 0.2$ & $68.9567 \pm 0.0539$ & $9.050 \pm 0.022$ & $0.526 \pm 0.032$ & & & \\
\hline Gl 891 & 9.75 & 1.046 & 3735 & 3779 & $3757 \pm 22$ & dM1.0 & $67.0946 \pm 0.0516$ & $8.883 \pm 0.022$ & $0.637 \pm 0.031$ & -0.13 & & \\
\hline Gl 894.1 & 10.922 & 1.030 & 3749 & 3794 & $3771 \pm 23$ & dM0.9 & $42.1139 \pm 0.0313$ & $9.044 \pm 0.022$ & $0.582 \pm 0.029$ & & & \\
\hline HIP 34730 & 12.235 & 1.097 & 3688 & - & $3688 \pm 22$ & $\mathrm{dM} 1.5$ & $27.9890 \pm 0.0649$ & $9.470 \pm 0.025$ & $0.526 \pm 0.027$ & -0.67 & & \\
\hline HIP 61704 & 11.485 & 0.965 & 3806 & 3799 & $3803 \pm 4$ & dM0.6 & $20.9087 \pm 0.0287$ & $8.087 \pm 0.023$ & $0.872 \pm 0.023$ & & & Binary? \\
\hline HIP 65083 & 11.601 & 1.110 & 3676 & 3715 & $3696 \pm 19$ & dM1.5 & $44.3846 \pm 0.0526$ & $9.837 \pm 0.023$ & $0.440 \pm 0.020$ & -0.37 & & Subdwarf? \\
\hline HIP 91724 & 11.6 & 1.097 & 3688 & - & $3688 \pm 22$ & $\mathrm{dM} 1.5$ & $25.1354 \pm 0.0221$ & $8.601 \pm 0.202$ & $0.785 \pm 0.110$ & +0.10 & & Binary? \\
\hline HIP 110980 & 10.514 & 0.946 & 3822 & 3803 & $3812 \pm 9$ & $\mathrm{dM} 0.5$ & $45.0944 \pm 0.0898$ & $8.785 \pm 0.024$ & $0.626 \pm 0.021$ & & & \\
\hline G $82-29$ & 11.535 & 1.046 & 3735 & 3691 & $3713 \pm 22$ & $\mathrm{dM} 1.3$ & $38.0213 \pm 0.0484$ & $9.435 \pm 0.023$ & $0.519 \pm 0.026$ & +0.12 & & \\
\hline G $182-37$ & 10.96 & 1.046 & 3735 & 3680 & $3708 \pm 28$ & $\mathrm{dM} 1.4$ & $46.2650 \pm 0.0220$ & $9.286 \pm 0.021$ & $0.559 \pm 0.032$ & +0.37 & & \\
\hline G $192-11 \mathrm{~A}$ & 10.255 & 1.076 & 3707 & 3819 & $3763 \pm 56$ & $\mathrm{dM} 1.0$ & $74.1524 \pm 0.0432$ & $9.606 \pm 0.021$ & $0.453 \pm 0.041$ & & & Subdwarf? \\
\hline MCC 197 & 10.28 & 1.084 & 3700 & 3830 & $3765 \pm 65$ & sdM0.9 & $71.7677 \pm 0.0559$ & $9.560 \pm 0.022$ & $0.462 \pm 0.047$ & -1.00 & & \\
\hline MCC $452 \mathrm{~A}$ & 11.017 & 1.046 & 3735 & 3771 & $3753 \pm 18$ & $\mathrm{dM} 1.0$ & $49.2062 \pm 0.0572$ & $9.477 \pm 0.023$ & $0.486 \pm 0.021$ & -0.06 & 5 & \\
\hline MCC 452B & 11.152 & 1.046 & 3735 & 3771 & $3753 \pm 18$ & $\mathrm{dM} 1.0$ & $49.1614 \pm 0.0445$ & $9.610 \pm 0.022$ & $0.458 \pm 0.020$ & -0.06 & 5 & \\
\hline MCC 488 & 11.20 & 1.076 & 3707 & 3701 & $3704 \pm 3$ & $\mathrm{dM} 1.4$ & $39.6119 \pm 0.0439$ & $9.189 \pm 0.022$ & $0.588 \pm 0.016$ & & & \\
\hline STKM 1-112 & 11.88 & 1.034 & 3746 & 3739 & $3743 \pm 3$ & $\mathrm{dM} 1.1$ & $28.6308 \pm 0.0506$ & $9.164 \pm 0.024$ & $0.568 \pm 0.015$ & +0.03 & & \\
\hline STKM 1-376 & 11.59 & 0.980 & 3793 & 3691 & $3742 \pm 53$ & dM1.1 & $20.9971 \pm 0.0406$ & $8.201 \pm 0.024$ & $0.887 \pm 0.078$ & & & Binary? \\
\hline
\end{tabular}


Table 2:: continued.

\begin{tabular}{|c|c|c|c|c|c|c|c|c|c|c|c|c|}
\hline Star & $\begin{array}{c}\mathrm{V} \\
(\mathrm{mag})\end{array}$ & $\begin{array}{c}(\mathrm{R}-\mathrm{I})_{C} \\
(\mathrm{mag})\end{array}$ & $\begin{array}{c}T_{\text {eff }}{ }^{1} \\
(\mathrm{~K})\end{array}$ & $\begin{array}{c}T_{e f f}{ }^{2} \\
(\mathrm{~K})\end{array}$ & $\begin{array}{c}T_{e f f} \pm 3 \sigma^{3} \\
(\mathrm{~K})\end{array}$ & $\begin{array}{l}\text { Spect. } \\
\text { Type }\end{array}$ & $\begin{array}{c}\pi \\
(\mathrm{m} ")\end{array}$ & $\begin{array}{c}M_{V} \\
\text { (mag) }\end{array}$ & $\begin{array}{c}R_{\star} \\
\left(R_{\odot}\right)\end{array}$ & {$[\mathrm{M} / \mathrm{H}]$} & $\begin{array}{l}\text { Hi. Res. } \\
\text { Spectra }\end{array}$ & Comments \\
\hline STKM 1-497 & 10.59 & 1.112 & 3674 & 3698 & $3686 \pm 12$ & $\mathrm{dM} 1.5 \mathrm{e}$ & $37.9633 \pm 0.3662$ & $8.487 \pm 0.041$ & $0.829 \pm 0.039$ & & 2 & PMS? \\
\hline STKM 1-1497A & 13.512 & 1.046 & 3735 & - & $3735 \pm 30$ & $\mathrm{dM} 1.2$ & $17.4699 \pm 0.0466$ & $9.723 \pm 0.026$ & $0.443 \pm 0.027$ & & & Subdwarf? \\
\hline STKM 1-1497B & 13.512 & 1.046 & 3735 & - & $3735 \pm 30$ & $\mathrm{dM} 1.2$ & $17.4699 \pm 0.0466$ & $9.723 \pm 0.026$ & $0.443 \pm 0.027$ & & & Subdwarf? \\
\hline LHS 321 & 12.360 & 1.047 & 3734 & - & $3734 \pm 22$ & $\mathrm{dM} 1.2$ & $30.1278 \pm 0.1384$ & $9.755 \pm 0.030$ & $0.437 \pm 0.023$ & & & Subdwarf? \\
\hline LHS 1637 & 11.68 & 1.097 & 3688 & 3704 & $3696 \pm 8$ & $\mathrm{dM} 1.5$ & $30.9958 \pm 0.0432$ & $9.137 \pm 0.023$ & $0.608 \pm 0.020$ & -0.38 & & \\
\hline LHS 2329 & 12.15 & 1.070 & 3713 & - & $3713 \pm 22$ & $\mathrm{dM} 1.3$ & $31.6083 \pm 0.0314$ & $9.649 \pm 0.022$ & $0.471 \pm 0.023$ & & & \\
\hline LHS 3166 & 12.977 & 1.046 & 3735 & - & $3735 \pm 22$ & $\mathrm{dM} 1.2$ & $23.7238 \pm 0.0203$ & $9.853 \pm 0.022$ & $0.418 \pm 0.020$ & & & Subdwarf? \\
\hline LHS 3214 & 11.863 & 1.046 & 3735 & 3728 & $3732 \pm 4$ & $\mathrm{dM} 1.2$ & $33.1218 \pm 0.0217$ & $9.464 \pm 0.021$ & $0.501 \pm 0.014$ & -0.11 & & \\
\hline LHS 3519 & 12.834 & 1.050 & 3731 & - & $3731 \pm 22$ & $\mathrm{dM} 1.2$ & $24.8879 \pm 0.0354$ & $9.814 \pm 0.023$ & $0.427 \pm 0.021$ & & & Subdwarf? \\
\hline LHS 3943 & 12.070 & 1.065 & 3717 & 3685 & $3701 \pm 16$ & $\mathrm{dM} 1.4$ & $27.7006 \pm 0.0694$ & $9.282 \pm 0.025$ & $0.565 \pm 0.025$ & & & \\
\hline LDS $3000 \mathrm{~A}$ & 12.381 & 1.097 & 3688 & - & $3688 \pm 22$ & $\mathrm{dM} 1.5$ & $20.7130 \pm 0.0706$ & $8.962 \pm 0.027$ & $0.664 \pm 0.035$ & & & \\
\hline LDS 3315A & 13.71 & 1.072 & 3711 & - & $3711 \pm 30$ & $\mathrm{dM} 1.3$ & $15.0618 \pm 0.1519$ & $9.599 \pm 0.042$ & $0.483 \pm 0.033$ & & & \\
\hline LDS 3315B & 14.03 & 1.072 & 3711 & - & $3711 \pm 30$ & $\mathrm{sdM} 1.3$ & $15.3218 \pm 0.0411$ & $9.957 \pm 0.026$ & $0.409 \pm 0.025$ & & & Subdwarf? \\
\hline LP $437-54$ & 15.22 & 1.135 & 3651 & 3851 & $3751 \pm 100$ & $\mathrm{dM} 1.0$ & $6.7792 \pm 0.0345$ & $9.376 \pm 0.031$ & $0.511 \pm 0.078$ & -0.47 & & \\
\hline L $1298-82$ & 10.84 & 1.046 & 3734 & 3708 & $3721 \pm 13$ & $\mathrm{dM} 1.3$ & $36.1378 \pm 0.0381$ & $8.630 \pm 0.022$ & $0.745 \pm 0.029$ & & & Binary? \\
\hline & & & & & dM2 sample & & & & & & & \\
\hline GJ & & & & & \pm & $\mathrm{dM}$ & \pm & \pm & \pm & & & \\
\hline GJ 1009 & 11.153 & 1.217 & 3571 & 3587 & $3579 \pm 8$ & $\mathrm{dM} 2.3$ & $55.3900 \pm 0.0379$ & $9.870 \pm 0.021$ & $0.496 \pm 0.017$ & & & \\
\hline GJ 1010A & 11.325 & 1.148 & 3638 & 3592 & $3615 \pm 23$ & $\mathrm{dM} 2.1$ & $52.2518 \pm 0.0209$ & $9.916 \pm 0.021$ & $0.466 \pm 0.024$ & +0.01 & 1 & \\
\hline GJ 1051 & 11.914 & 1.103 & 3683 & 3586 & $3634 \pm 48$ & $\mathrm{dM} 1.9$ & $46.8824 \pm 0.0611$ & $10.269 \pm 0.023$ & $0.387 \pm 0.032$ & -0.31 & 5 & \\
\hline GJ 1062 & 13.015 & 1.184 & 3602 & - & $3602 \pm 30$ & $\operatorname{sdM} 2.1$ & $61.8726 \pm 0.0499$ & $11.972 \pm 0.022$ & $0.184 \pm 0.011$ & & & Subdwarf? \\
\hline GJ 1114 & 11.523 & 1.148 & 3638 & 3519 & $3578 \pm 59$ & $\operatorname{sdM} 2.3$ & $59.7215 \pm 0.0581$ & $10.404 \pm 0.022$ & $0.389 \pm 0.038$ & -0.51 & 4 & \\
\hline GJ $1141 \mathrm{~A}$ & 11.493 & 1.084 & 3700 & 3665 & $3683 \pm 18$ & $\mathrm{dM} 1.6$ & $38.9589 \pm 0.0621$ & $9.446 \pm 0.023$ & $0.535 \pm 0.024$ & & & \\
\hline GJ 1141B & 11.623 & 1.123 & 3663 & 3637 & $3650 \pm 13$ & $\mathrm{dM} 1.8$ & $38.9784 \pm 0.0617$ & $9.577 \pm 0.023$ & $0.523 \pm 0.021$ & & & \\
\hline GJ 1248 & 12.142 & 1.224 & 3565 & 3436 & $3501 \pm 64$ & sdM2.7 & $75.3873 \pm 0.0597$ & $11.528 \pm 0.022$ & $0.254 \pm 0.027$ & -0.34 & & Subdwarf? \\
\hline GJ 1264 & 9.641 & 1.112 & 3674 & 3694 & $3684 \pm 10$ & PMS M1.6e & $60.4905 \pm 0.3353$ & $8.549 \pm 0.032$ & $0.807 \pm 0.032$ & & 3 & \\
\hline GJ 1266 & 12.045 & 1.174 & 3612 & 3572 & $3592 \pm 20$ & $\mathrm{dM} 2.2$ & $43.7721 \pm 0.0262$ & $10.251 \pm 0.021$ & $0.410 \pm 0.020$ & & 2 & \\
\hline GJ 1274 & 11.72 & 1.186 & 3600 & - & $3600 \pm 30$ & $\mathrm{dM} 2.1$ & $51.8108 \pm 0.1092$ & $10.292 \pm 0.025$ & $0.399 \pm 0.025$ & & 1 & \\
\hline GJ 2066 & 10.109 & 1.227 & 3562 & 3546 & $3554 \pm 8$ & $\mathrm{dM} 2.4 \mathrm{e}$ & $111.8419 \pm 0.0534$ & $10.352 \pm 0.021$ & $0.409 \pm 0.014$ & -0.12 & 1 & \\
\hline GJ 2085 & 11.185 & 1.058 & 3724 & 3592 & $3658 \pm 66$ & $\mathrm{dM} 1.8$ & $49.6262 \pm 0.0385$ & $9.664 \pm 0.022$ & $0.498 \pm 0.053$ & & & \\
\hline GJ 2116 & 13.10 & 1.129 & 3657 & 3600 & $3628 \pm 28$ & $\operatorname{sdM} 2.0$ & $42.8010 \pm 0.0408$ & $11.257 \pm 0.022$ & $0.248 \pm 0.014$ & -0.60 & 1 & \\
\hline GJ 3014 & 11.015 & 1.184 & 3602 & 3607 & $3605 \pm 2$ & $\mathrm{dM} 2.1$ & $51.9505 \pm 0.0270$ & $9.593 \pm 0.021$ & $0.547 \pm 0.014$ & +0.99 & 5 & \\
\hline GJ 3023 & 11.525 & 1.155 & 3631 & 3602 & $3617 \pm 15$ & $\mathrm{dM} 2.1$ & $43.7264 \pm 0.0400$ & $9.729 \pm 0.022$ & $0.507 \pm 0.021$ & +0.18 & 2 & \\
\hline GJ 3084 & 10.791 & 1.064 & 3718 & 3620 & $3669 \pm 49$ & $\mathrm{dM} 1.7$ & $55.2084 \pm 0.0527$ & $9.501 \pm 0.022$ & $0.530 \pm 0.044$ & -0.29 & 2 & \\
\hline GJ 3098 & 11.155 & 1.114 & 3672 & 3582 & $3627 \pm 45$ & $\mathrm{dM} 2.0$ & $53.7324 \pm 0.0354$ & $9.806 \pm 0.021$ & $0.483 \pm 0.038$ & -0.36 & 12 & \\
\hline GJ 3103 & 11.562 & 1.188 & 3598 & 3570 & $3584 \pm 14$ & $\mathrm{dM} 2.2$ & $48.2229 \pm 0.0951$ & $9.978 \pm 0.024$ & $0.470 \pm 0.020$ & +0.11 & & \\
\hline GJ 3207 & 11.51 & 1.160 & 3626 & 3445 & $3535 \pm 90$ & $\mathrm{dM} 2.5$ & $44.3268 \pm 0.0296$ & $9.743 \pm 0.021$ & $0.554 \pm 0.078$ & -0.11 & 6 & Binary? \\
\hline GJ 3215 & 11.779 & 1.148 & 3638 & 3525 & $3581 \pm 56$ & $\mathrm{dM} 2.3$ & $46.0534 \pm 0.0499$ & $10.095 \pm 0.022$ & $0.446 \pm 0.042$ & & 1 & \\
\hline GJ 3218 & 11.314 & 1.224 & 3565 & 3670 & $3618 \pm 53$ & $\mathrm{dM} 2.0$ & $58.0400 \pm 0.0515$ & $10.133 \pm 0.022$ & $0.420 \pm 0.038$ & -0.47 & & \\
\hline GJ 3248 & 11.03 & 1.135 & 3651 & 3581 & $3616 \pm 35$ & $\mathrm{dM} 2.1$ & $62.7050 \pm 0.0283$ & $10.017 \pm 0.021$ & $0.444 \pm 0.030$ & +1.08 & 2 & \\
\hline GJ 3256 & 11.225 & 1.212 & 3575 & 3699 & $3637 \pm 62$ & dM1.9 & $47.3869 \pm 0.0638$ & $9.603 \pm 0.023$ & $0.525 \pm 0.053$ & -0.12 & & \\
\hline GJ 3307 & 11.228 & 1.135 & 3651 & 3595 & $3623 \pm 28$ & $\mathrm{dM} 2.0$ & $41.8298 \pm 0.1755$ & $9.335 \pm 0.029$ & $0.603 \pm 0.037$ & +0.84 & & \\
\hline GJ 3321 & 11.291 & 1.097 & 3688 & 3645 & $3667 \pm 21$ & $\mathrm{dM} 1.7$ & $53.0273 \pm 0.0396$ & $9.913 \pm 0.022$ & $0.439 \pm 0.021$ & +0.01 & 1 & \\
\hline GJ 3331 & 10.41 & 1.148 & 3638 & 3685 & $3662 \pm 23$ & $\mathrm{dM} 1.7$ & $50.3080 \pm 0.0357$ & $8.918 \pm 0.022$ & $0.699 \pm 0.036$ & & & Triple system \\
\hline GJ 3340 & 11.542 & 1.070 & 3713 & 3653 & $3683 \pm 30$ & $\mathrm{dM} 1.6$ & $46.4015 \pm 0.0854$ & $9.875 \pm 0.024$ & $0.439 \pm 0.027$ & & 2 & \\
\hline GJ 3385 & 11.36 & 1.134 & 3652 & 3640 & $3646 \pm 6$ & $\mathrm{dM} 1.9$ & $37.3371 \pm 0.3689$ & $9.221 \pm 0.041$ & $0.619 \pm 0.025$ & & 1 & Binary? \\
\hline GJ 3395 & 11.077 & 1.072 & 3711 & 3599 & $3655 \pm 56$ & $\mathrm{dM} 1.8$ & $48.3803 \pm 0.0364$ & $9.500 \pm 0.022$ & $0.539 \pm 0.050$ & & 1 & \\
\hline GJ 3414 & 12.435 & 1.199 & 3587 & 3380 & $3484 \pm 104$ & $\mathrm{dM} 2.8$ & $46.5826 \pm 0.0540$ & $10.776 \pm 0.023$ & $0.370 \pm 0.091$ & & 1 & Subdwarf? \\
\hline GJ 3425 & 11.165 & 1.123 & 3663 & 3674 & $3669 \pm 5$ & $\mathrm{dM} 1.7$ & $52.6548 \pm 0.1049$ & $9.772 \pm 0.024$ & $0.468 \pm 0.014$ & +0.75 & 1 & \\
\hline
\end{tabular}


Table 2:: continued.

\begin{tabular}{|c|c|c|c|c|c|c|c|c|c|c|c|c|}
\hline Star & $\begin{array}{c}\mathrm{V} \\
(\mathrm{mag})\end{array}$ & $\begin{array}{c}(\mathrm{R}-\mathrm{I})_{C} \\
(\mathrm{mag})\end{array}$ & $\begin{array}{c}T_{\text {eff }}{ }^{1} \\
(\mathrm{~K})\end{array}$ & $\begin{array}{c}T_{e f f}{ }^{2} \\
(\mathrm{~K})\end{array}$ & $\begin{array}{c}T_{\text {eff }} \pm 3 \sigma^{3} \\
\quad(\mathrm{~K})\end{array}$ & $\begin{array}{l}\text { Spect. } \\
\text { Type }\end{array}$ & $\begin{array}{c}\pi \\
(\mathrm{m} ")\end{array}$ & $\begin{array}{c}M_{V} \\
(\mathrm{mag})\end{array}$ & $\begin{array}{c}R_{\star} \\
\left(R_{\odot}\right)\end{array}$ & {$[\mathrm{M} / \mathrm{H}]$} & $\begin{array}{l}\text { Hi. Res. } \\
\text { Spectra }\end{array}$ & Comments \\
\hline GJ 3440 & 11.75 & 1.148 & 3638 & 3530 & $3584 \pm 54$ & $\mathrm{dM} 2.2$ & $36.4088 \pm 0.0876$ & $9.556 \pm 0.025$ & $0.570 \pm 0.054$ & & 28 & \\
\hline GJ 3462 & 11.305 & 1.115 & 3671 & 3641 & $3656 \pm 15$ & $\mathrm{dM} 1.8$ & $30.8479 \pm 0.0540$ & $8.751 \pm 0.024$ & $0.760 \pm 0.032$ & -0.25 & 1 & Binary? \\
\hline GJ 3536 & 11.92 & 1.123 & 3663 & 3536 & $3600 \pm 63$ & $\mathrm{dM} 2.1$ & $38.5 \pm 9.2$ & $9.847 \pm 0.485$ & $0.490 \pm 0.179$ & -0.11 & & \\
\hline GJ 3543 & 10.55 & 1.186 & 3600 & 3698 & $3649 \pm 49$ & $\mathrm{dM} 2.1$ & $74.3573 \pm 0.0398$ & $9.907 \pm 0.021$ & $0.450 \pm 0.038$ & -0.08 & 103 & \\
\hline GJ 3560 & 11.929 & 1.237 & 3553 & 3554 & $3553 \pm 1$ & $\mathrm{dM} 2.4$ & $50.3508 \pm 0.0471$ & $10.439 \pm 0.022$ & $0.394 \pm 0.010$ & +1.21 & & \\
\hline GJ 3649 & 10.81 & 1.123 & 3663 & - & $3663 \pm 30$ & sdM1.7 & $59.9389 \pm 0.0527$ & $9.699 \pm 0.022$ & $0.487 \pm 0.029$ & -1.11 & & Binary? \\
\hline GJ 3671 & 11.166 & 1.106 & 3680 & - & $3680 \pm 30$ & sdM1.6 & $54.7349 \pm 0.0545$ & $9.857 \pm 0.022$ & $0.444 \pm 0.027$ & -0.54 & & \\
\hline GJ 3695 & 11.867 & 1.186 & 3600 & 3635 & $3617 \pm 18$ & $\mathrm{dM} 2.0$ & $39.5898 \pm 0.1050$ & $9.855 \pm 0.026$ & $0.478 \pm 0.023$ & +0.03 & & \\
\hline GJ 3714 & 11.362 & 1.123 & 3663 & 3652 & $3658 \pm 6$ & $\mathrm{dM} 1.8$ & $31.7979 \pm 0.3252$ & $8.874 \pm 0.042$ & $0.716 \pm 0.029$ & +0.56 & & Binary? \\
\hline GJ 3778 & 11.9 & 1.186 & 3600 & 3493 & $3546 \pm 53$ & $\mathrm{dM} 2.5$ & $43.3772 \pm 0.0484$ & $10.086 \pm 0.202$ & $0.467 \pm 0.087$ & & 18 & \\
\hline GJ 3809 & 11.195 & 1.199 & 3587 & 3591 & $3589 \pm 2$ & $\operatorname{sdM} 2.2$ & $55.7827 \pm 0.7529$ & $9.927 \pm 0.049$ & $0.478 \pm 0.019$ & -0.73 & & \\
\hline GJ 3823 & 12.15 & 1.216 & 3572 & 3514 & $3543 \pm 29$ & $\mathrm{dM} 2.5$ & $55.4066 \pm 0.0625$ & $10.868 \pm 0.022$ & $0.327 \pm 0.020$ & -0.33 & & Subdwarf? \\
\hline GJ 3826 & 11.551 & 1.084 & 3700 & 3616 & $3658 \pm 42$ & $\mathrm{dM} 1.8$ & $43.2202 \pm 0.2319$ & $9.729 \pm 0.032$ & $0.483 \pm 0.039$ & & 1 & \\
\hline GJ 3890 & 10.982 & 1.119 & 3667 & 3663 & $3665 \pm 2$ & $\mathrm{dM} 1.7$ & $58.4575 \pm 0.0296$ & $9.816 \pm 0.021$ & $0.460 \pm 0.012$ & -0.05 & & \\
\hline GJ 3915 & 11.65 & 1.132 & 3654 & 3578 & $3616 \pm 38$ & $\mathrm{dM} 2.1$ & $37.4001 \pm 0.8200$ & $9.514 \pm 0.067$ & $0.560 \pm 0.052$ & -0.46 & 5 & Binary? \\
\hline GJ 3920 & 12.046 & 1.237 & 3553 & 3502 & $3528 \pm 26$ & $\mathrm{dM} 2.6$ & $47.4734 \pm 0.0301$ & $10.428 \pm 0.021$ & $0.408 \pm 0.023$ & & 1 & \\
\hline GJ 3968 & 11.519 & 1.186 & 3600 & 3620 & $3610 \pm 10$ & $\mathrm{sdM} 2.1$ & $46.5739 \pm 0.0391$ & $9.860 \pm 0.022$ & $0.481 \pm 0.017$ & -1.08 & & Binary? \\
\hline GJ 3973 & 10.914 & 1.123 & 3663 & 3690 & $3676 \pm 14$ & $\mathrm{dM} 1.6$ & $52.7356 \pm 0.0506$ & $9.525 \pm 0.022$ & $0.520 \pm 0.021$ & -0.07 & & \\
\hline GJ 4091A & 12.720 & 1.186 & 3600 & 3667 & $3634 \pm 34$ & $\mathrm{dM} 1.9$ & $23.6502 \pm 0.0712$ & $9.589 \pm 0.027$ & $0.530 \pm 0.036$ & +0.14 & 1 & \\
\hline GJ 4091B & 12.720 & 1.186 & 3600 & 3667 & $3634 \pm 34$ & $\mathrm{dM} 1.9$ & $23.6502 \pm 0.0712$ & $9.589 \pm 0.027$ & $0.530 \pm 0.036$ & +0.14 & 1 & \\
\hline GJ 4099 & 11.345 & 1.135 & 3651 & 3631 & $3641 \pm 10$ & dM1.9 & $36.0339 \pm 0.1196$ & $9.129 \pm 0.027$ & $0.650 \pm 0.025$ & +0.41 & 1 & \\
\hline GJ 4155 & 12.10 & 1.184 & 3602 & 3567 & $3584 \pm 17$ & $\mathrm{dM} 2.3$ & $44 \pm 8$ & $10.317 \pm 0.383$ & $0.402 \pm 0.094$ & -0.11 & 1 & \\
\hline GJ 4170 & 11.640 & 1.097 & 3688 & 3556 & $3622 \pm 66$ & $\mathrm{dM} 2.0$ & $45.8242 \pm 0.2330$ & $9.945 \pm 0.031$ & $0.456 \pm 0.051$ & -0.38 & 1 & \\
\hline GJ 4249 & 12.098 & 1.223 & 3566 & - & $3566 \pm 30$ & $\mathrm{dM} 2.3$ & $47.6632 \pm 0.0909$ & $10.489 \pm 0.024$ & $0.379 \pm 0.024$ & & & \\
\hline GJ 4308 & 11.559 & 1.110 & 3676 & - & $3676 \pm 30$ & dM1.6 & $50.7782 \pm 0.0635$ & $10.087 \pm 0.023$ & $0.401 \pm 0.024$ & & & \\
\hline GJ 4309 & 11.592 & 1.123 & 3663 & - & $3663 \pm 30$ & $\mathrm{dM} 1.7$ & $50.7570 \pm 0.0453$ & $10.119 \pm 0.022$ & $0.401 \pm 0.024$ & & & \\
\hline GJ 4311 & 11.78 & 1.212 & 3575 & - & $3575 \pm 30$ & $\mathrm{dM} 2.3$ & $55.3014 \pm 0.0459$ & $10.494 \pm 0.022$ & $0.374 \pm 0.023$ & & 1 & Subdwarf? \\
\hline GJ 4352 & 11.314 & 1.224 & 3565 & - & $3565 \pm 30$ & $\mathrm{dM} 2.3$ & $76.0557 \pm 0.0679$ & $10.720 \pm 0.022$ & $0.341 \pm 0.021$ & & & Subdwarf? \\
\hline GJ 9050 & 11.803 & 1.223 & 3566 & 3546 & $3556 \pm 10$ & $\mathrm{dM} 2.4$ & $42.0987 \pm 0.0568$ & $9.924 \pm 0.023$ & $0.497 \pm 0.019$ & +0.01 & & \\
\hline GJ 9201 & 12.145 & 1.186 & 3600 & 3513 & $3556 \pm 43$ & $\mathrm{dM} 2.4$ & $33.4805 \pm 0.0267$ & $9.769 \pm 0.022$ & $0.534 \pm 0.042$ & & 22 & \\
\hline GJ 9381 & 11.56 & 1.148 & 3638 & 3614 & $3626 \pm 12$ & $\mathrm{dM} 2.0$ & $39.1208 \pm 0.0476$ & $9.522 \pm 0.023$ & $0.552 \pm 0.021$ & -0.26 & 1 & \\
\hline GJ 9428 & 11.725 & 1.142 & 3644 & 3625 & $3635 \pm 10$ & $\mathrm{dM} 1.9$ & $38.2410 \pm 0.6800$ & $9.638 \pm 0.058$ & $0.518 \pm 0.028$ & -0.11 & & \\
\hline GJ 9520 & 10.003 & 1.175 & 3611 & - & $3611 \pm 30$ & $\mathrm{dM} 2.1$ & $87.3783 \pm 0.0493$ & $9.710 \pm 0.021$ & $0.515 \pm 0.031$ & & 9 & \\
\hline GJ 9674 & 11.835 & 1.097 & 3688 & 3645 & $3667 \pm 21$ & $\mathrm{dM} 1.7$ & $39.4015 \pm 0.0311$ & $9.813 \pm 0.022$ & $0.460 \pm 0.022$ & & 1 & \\
\hline Gl 1 & 8.560 & 1.166 & 3620 & 3537 & $3578 \pm 41$ & $\mathrm{dM} 2.3$ & $230.1331 \pm 0.0600$ & $10.370 \pm 0.021$ & $0.395 \pm 0.030$ & -0.45 & 74 & \\
\hline GL 2 & 9.949 & 1.112 & 3674 & 3623 & $3648 \pm 25$ & $\mathrm{dM} 1.8$ & $86.9567 \pm 0.0407$ & $9.646 \pm 0.021$ & $0.508 \pm 0.027$ & -0.00 & 10 & \\
\hline Gl $15 \mathrm{~A}$ & 8.13 & 1.130 & 3656 & 3632 & $3644 \pm 12$ & sdM1.9 & $280.7866 \pm 0.0519$ & $10.372 \pm 0.020$ & $0.365 \pm 0.014$ & -0.82 & 38 & \\
\hline Gl 16 & 10.864 & 1.148 & 3638 & 3691 & $3664 \pm 27$ & $\mathrm{dM} 1.7$ & $59.5952 \pm 0.0950$ & $9.740 \pm 0.023$ & $0.477 \pm 0.027$ & & 2 & \\
\hline GL 27.1 & 11.401 & 1.078 & 3705 & 3610 & $3658 \pm 48$ & $\mathrm{dM} 1.8$ & $42.3328 \pm 0.0456$ & $9.534 \pm 0.022$ & $0.529 \pm 0.044$ & +0.26 & 28 & \\
\hline Gl 29.1A & 11.146 & 1.136 & 3650 & 3658 & $3654 \pm 4$ & $\mathrm{dM} 1.8 \mathrm{e}$ & $46.0337 \pm 0.0542$ & $9.461 \pm 0.023$ & $0.549 \pm 0.016$ & +0.14 & & \\
\hline Gl $29.1 \mathrm{~B}$ & 11.146 & 1.136 & 3650 & 3658 & $3654 \pm 4$ & $\mathrm{dM} 1.8 \mathrm{e}$ & $46.0337 \pm 0.0542$ & $9.461 \pm 0.023$ & $0.549 \pm 0.016$ & +0.14 & & \\
\hline Gl 43 & 12.37 & 1.186 & 3600 & 3568 & $3584 \pm 16$ & $\mathrm{dM} 2.2$ & $33.5361 \pm 0.0316$ & $9.998 \pm 0.022$ & $0.465 \pm 0.020$ & & 17 & \\
\hline Gl 49 & 9.564 & 1.148 & 3638 & 3649 & $3643 \pm 6$ & dM1.9 & $101.4650 \pm 0.0335$ & $9.596 \pm 0.021$ & $0.523 \pm 0.016$ & +0.08 & 4 & \\
\hline Gl 56.2 & 11.684 & 1.229 & 3560 & 3716 & $3638 \pm 78$ & $\mathrm{dM} 1.9$ & $20.0402 \pm 0.4931$ & $8.194 \pm 0.073$ & $1.003 \pm 0.149$ & +0.16 & & PMS? \\
\hline Gl 78 & 11.791 & 1.210 & 3577 & 3580 & $3579 \pm 1$ & $\mathrm{dM} 2.3$ & $58.1554 \pm 0.0521$ & $10.614 \pm 0.022$ & $0.352 \pm 0.009$ & -0.24 & & Subdwarf? \\
\hline Gl 85 & 11.55 & - & - & 3487 & $3487 \pm 30$ & $\mathrm{dM} 2.8$ & $81.2409 \pm 0.0319$ & $11.099 \pm 0.021$ & $0.317 \pm 0.027$ & & 10 & Subdwarf? \\
\hline Gl 87 & 10.042 & 1.127 & 3659 & 3592 & $3625 \pm 33$ & $\mathrm{dM} 2.0$ & $95.1619 \pm 0.0413$ & $9.934 \pm 0.021$ & $0.457 \pm 0.029$ & -0.34 & 96 & \\
\hline Gl 91 & 10.324 & 1.175 & 3611 & 3663 & $3637 \pm 26$ & $\mathrm{dM} 1.9$ & $78.1936 \pm 0.0372$ & $9.790 \pm 0.021$ & $0.481 \pm 0.027$ & -0.49 & 27 & \\
\hline Gl 104 & 10.642 & 1.213 & 3574 & 3592 & $3583 \pm 9$ & $\mathrm{dM} 2.2$ & $71.1607 \pm 0.0684$ & $9.903 \pm 0.022$ & $0.487 \pm 0.017$ & -0.31 & 1 & \\
\hline Gl 107B & 9.987 & 1.160 & 3626 & 3632 & $3629 \pm 3$ & $\mathrm{dM} 2.0$ & $89.6955 \pm 0.3006$ & $9.751 \pm 0.027$ & $0.495 \pm 0.015$ & & & \\
\hline GL $114.1 \mathrm{~A}$ & 10.717 & 1.181 & 3605 & 3607 & $3606 \pm 1$ & $\mathrm{dM} 2.1$ & $75.7188 \pm 0.0240$ & $10.113 \pm 0.021$ & $0.430 \pm 0.011$ & +0.27 & 1 & \\
\hline Gl 125 & 10.164 & 1.199 & 3587 & 3515 & $3551 \pm 36$ & $\mathrm{dM} 2.4$ & $57.1088 \pm 0.8198$ & $8.948 \pm 0.051$ & $0.785 \pm 0.066$ & & 1 & Binary? \\
\hline
\end{tabular}


Table 2:: continued.

\begin{tabular}{|c|c|c|c|c|c|c|c|c|c|c|c|c|}
\hline Star & $\underset{(\mathrm{mag})}{\mathrm{V}}$ & $\begin{array}{c}(\mathrm{R}-\mathrm{I})_{C} \\
(\mathrm{mag})\end{array}$ & $\begin{array}{c}T_{\text {eff }}{ }^{1} \\
(\mathrm{~K})\end{array}$ & $\begin{array}{c}T_{e f f}^{2} \\
(\mathrm{~K})\end{array}$ & $T_{\text {eff }} \pm 3 \sigma^{3}$ & $\begin{array}{l}\text { Spect. } \\
\text { Type }\end{array}$ & $\begin{array}{c}\pi \\
(\mathrm{m} ")\end{array}$ & $\begin{array}{c}M_{V} \\
\text { (mag) }\end{array}$ & $\begin{array}{c}R_{\star} \\
\left(R_{\odot}\right)\end{array}$ & {$[\mathrm{M} / \mathrm{H}]$} & $\begin{array}{l}\text { Hi. Res. } \\
\text { Spectra }\end{array}$ & Comments \\
\hline Gl 130 & 11.51 & 1.160 & 3626 & 3561 & $3593 \pm 32$ & $\operatorname{sdM} 2.2$ & $69.4776 \pm 0.0696$ & $10.719 \pm 0.022$ & $0.330 \pm 0.021$ & -0.03 & & Subdwarf? \\
\hline Gl $130.1 \mathrm{~A}$ & 10.76 & 1.212 & 3575 & 3598 & $3586 \pm 12$ & $\mathrm{dM} 2.2$ & $73.7645 \pm 0.0442$ & $10.099 \pm 0.021$ & $0.443 \pm 0.017$ & +1.27 & 1 & \\
\hline Gl $130.1 \mathrm{~B}$ & 10.76 & 1.212 & 3575 & 3598 & $3586 \pm 12$ & $\mathrm{dM} 2.2$ & $73.7407 \pm 0.0417$ & $10.099 \pm 0.021$ & $0.443 \pm 0.017$ & +1.27 & 1 & \\
\hline Gl 133 & 11.21 & 1.208 & 3579 & 3456 & $3517 \pm 61$ & $\mathrm{dM} 2.6$ & $71.7575 \pm 0.0400$ & $10.489 \pm 0.021$ & $0.401 \pm 0.041$ & & 7 & \\
\hline Gl 134 & 10.29 & 1.124 & 3662 & 3608 & $3635 \pm 27$ & dM1.9 & $62.5647 \pm 0.0720$ & $9.272 \pm 0.022$ & $0.613 \pm 0.035$ & +0.53 & 6 & \\
\hline Gl $140 \mathrm{~A}$ & 11.175 & 1.160 & 3626 & 3599 & $3612 \pm 13$ & $\mathrm{dM} 2.1$ & $48.0921 \pm 0.0617$ & $9.585 \pm 0.023$ & $0.545 \pm 0.022$ & & 2 & \\
\hline Gl 140B & 11.175 & 1.160 & 3626 & 3599 & $3612 \pm 13$ & $\mathrm{dM} 2.1$ & $48.4516 \pm 0.0677$ & $9.602 \pm 0.023$ & $0.762 \pm 0.022$ & & 2 & \\
\hline Gl 150.1B & 10.763 & 1.112 & 3674 & 3626 & $3650 \pm 24$ & $\mathrm{dM} 1.8$ & $58.0070 \pm 0.0457$ & $9.580 \pm 0.022$ & $0.522 \pm 0.028$ & -0.03 & 3 & \\
\hline Gl 153B & 10.79 & 1.160 & 3626 & 3570 & $3598 \pm 28$ & $\mathrm{dM} 2.2$ & $52.3391 \pm 0.6475$ & $9.384 \pm 0.047$ & $0.607 \pm 0.043$ & +0.04 & 2 & \\
\hline Gl $153 \mathrm{C}$ & 10.79 & 1.160 & 3626 & 3570 & $3598 \pm 28$ & $\mathrm{dM} 2.2$ & $52.3391 \pm 0.6475$ & $9.384 \pm 0.047$ & $0.607 \pm 0.043$ & +0.04 & 2 & \\
\hline Gl 155.3 & 12.135 & 1.186 & 3600 & 3566 & $3583 \pm 17$ & $\mathrm{dM} 2.2$ & $39.1709 \pm 0.0386$ & $10.100 \pm 0.022$ & $0.445 \pm 0.020$ & & 18 & \\
\hline Gl 173 & 10.331 & 1.196 & 3590 & 3709 & $3649 \pm 60$ & $\mathrm{dM} 1.8$ & $89.1708 \pm 0.0313$ & $10.082 \pm 0.021$ & $0.415 \pm 0.041$ & +0.05 & 25 & \\
\hline Gl 176 & 9.972 & 1.250 & 3542 & 3599 & $3570 \pm 29$ & $\mathrm{dM} 2.3$ & $105.5627 \pm 0.0700$ & $10.090 \pm 0.021$ & $0.453 \pm 0.027$ & +0.05 & 23 & \\
\hline Gl 180 & 10.902 & 1.240 & 3551 & 3526 & $3538 \pm 12$ & $\mathrm{dM} 2.5$ & $83.7182 \pm 0.0313$ & $10.516 \pm 0.021$ & $0.387 \pm 0.015$ & -0.20 & & \\
\hline Gl 191 & 8.853 & 1.058 & 3724 & 3596 & $3660 \pm 64$ & sdM1.7 & $254.2263 \pm 0.0262$ & $10.879 \pm 0.020$ & $0.284 \pm 0.029$ & -0.80 & 22 & \\
\hline Gl 195A & 10.130 & 1.174 & 3612 & 3623 & $3618 \pm 5$ & $\mathrm{dM} 2.0$ & $75.0151 \pm 0.0406$ & $9.506 \pm 0.021$ & $0.561 \pm 0.017$ & -0.24 & & \\
\hline Gl 218 & 10.736 & 1.151 & 3635 & 3533 & $3584 \pm 51$ & $\mathrm{dM} 2.2$ & $68.9811 \pm 0.0258$ & $9.930 \pm 0.021$ & $0.480 \pm 0.042$ & & 13 & \\
\hline Gl 220 & 10.829 & 1.137 & 3649 & 3701 & $3675 \pm 26$ & dM1.6 & 59. \pm 7 & $9.683 \pm 0.263$ & $0.484 \pm 0.087$ & -0.15 & 1 & \\
\hline Gl 289 & 11.418 & 1.151 & 3635 & 3586 & $3610 \pm 24$ & $\operatorname{sdM} 2.1$ & $68.0346 \pm 0.0438$ & $10.582 \pm 0.021$ & $0.345 \pm 0.018$ & -0.31 & 5 & Subdwarf? \\
\hline Gl 330 & 10.593 & 1.100 & 3685 & 3542 & $3613 \pm 72$ & $\mathrm{dM} 2.1$ & $58.4208 \pm 0.9079$ & $9.426 \pm 0.053$ & $0.585 \pm 0.077$ & & 1 & \\
\hline Gl 333 & 12.28 & 1.161 & 3625 & 3629 & $3627 \pm 2$ & $\operatorname{sdM} 2.0$ & $47.8960 \pm 0.0434$ & $10.681 \pm 0.022$ & $0.323 \pm 0.008$ & & 11 & Subdwarf? \\
\hline Gl 361 & 10.370 & 1.208 & 3579 & 3520 & $3549 \pm 29$ & $\mathrm{dM} 2.4$ & $86.5522 \pm 0.0430$ & $10.056 \pm 0.021$ & $0.472 \pm 0.029$ & -0.11 & 85 & \\
\hline Gl 366 & 10.635 & 1.112 & 3674 & 3588 & $3631 \pm 43$ & dM1.9 & $63.3142 \pm 0.0302$ & $9.643 \pm 0.021$ & $0.519 \pm 0.040$ & & & \\
\hline Gl 367 & 9.979 & 1.193 & 3593 & 3612 & $3603 \pm 10$ & $\mathrm{sdM} 2.1$ & $106.2112 \pm 0.0317$ & $10.110 \pm 0.021$ & $0.432 \pm 0.015$ & -0.54 & & \\
\hline Gl 378 & 10.11 & 1.256 & 3537 & 3590 & $3563 \pm 27$ & $\mathrm{dM} 2.4$ & $66.8407 \pm 0.0322$ & $9.235 \pm 0.021$ & $0.678 \pm 0.039$ & & 5 & Binary? \\
\hline Gl 382 & 9.264 & 1.184 & 3602 & 3617 & $3609 \pm 8$ & $\mathrm{dM} 2.1$ & $129.7887 \pm 0.0838$ & $9.830 \pm 0.021$ & $0.488 \pm 0.016$ & +0.06 & 39 & \\
\hline Gl 390 & 10.139 & 1.116 & 3670 & 3592 & $3631 \pm 39$ & dM1.9 & $78.0003 \pm 0.0982$ & $9.599 \pm 0.023$ & $0.529 \pm 0.038$ & -0.03 & 35 & \\
\hline Gl 411 & 7.508 & 1.184 & 3602 & 3618 & $3610 \pm 8$ & $\mathrm{dM} 2.1$ & $392.64 \pm 0.67$ & $10.478 \pm 0.024$ & $0.362 \pm 0.013$ & -0.31 & 63 & Subdwarf? \\
\hline Gl $412 \mathrm{~A}$ & 8.798 & 1.092 & 3692 & 3597 & $3644 \pm 47$ & sdM1.9 & $204.0592 \pm 0.1687$ & $10.347 \pm 0.022$ & $0.370 \pm 0.030$ & -0.58 & & \\
\hline Gl 413.1 & 10.448 & 1.231 & 3559 & 3551 & $3555 \pm 4$ & $\mathrm{dM} 2.4$ & $92.7821 \pm 0.0538$ & $10.285 \pm 0.021$ & $0.422 \pm 0.012$ & -0.11 & & \\
\hline Gl 414B & 10.069 & 1.171 & 3615 & 3624 & $3620 \pm 5$ & $\operatorname{sdM} 2.0$ & $84.1971 \pm 0.0579$ & $9.695 \pm 0.021$ & $0.513 \pm 0.015$ & -0.78 & & Binary? \\
\hline Gl 433 & 9.813 & 1.160 & 3626 & 3543 & $3584 \pm 41$ & $\mathrm{dM} 2.2$ & $110.2908 \pm 0.0444$ & $10.026 \pm 0.021$ & $0.459 \pm 0.034$ & -0.17 & 199 & \\
\hline Gl 450 & 9.762 & 1.136 & 3650 & 3678 & $3664 \pm 14$ & dM1.7 & $114.1376 \pm 0.0390$ & $10.049 \pm 0.021$ & $0.414 \pm 0.016$ & -0.11 & 7 & \\
\hline Gl 476 & 11.408 & 1.229 & 3560 & 3551 & $3555 \pm 5$ & $\operatorname{sdM} 2.4$ & $55.0431 \pm 0.0459$ & $10.112 \pm 0.022$ & $0.457 \pm 0.014$ & -0.86 & & \\
\hline Gl 477 & 11.092 & 1.190 & 3596 & 3592 & $3594 \pm 2$ & $\mathrm{dM} 2.2$ & $48.2102 \pm 0.5963$ & $9.508 \pm 0.047$ & $0.576 \pm 0.022$ & & 16 & \\
\hline Gl $489 \mathrm{~A}$ & 9.118 & 1.161 & 3625 & - & $3625 \pm 30$ & $\mathrm{dM} 2.0$ & $39.0038 \pm 0.3925$ & $7.074 \pm 0.042$ & $1.706 \pm 0.120$ & -0.09 & & PMS? \\
\hline Gl $490 \mathrm{~A}$ & 10.57 & 1.088 & 3696 & 3592 & $3644 \pm 52$ & $\mathrm{dM} 1.9 \mathrm{e}$ & $46.8354 \pm 0.2763$ & $8.923 \pm 0.033$ & $0.712 \pm 0.067$ & +0.07 & & PMS? \\
\hline Gl $494 \mathrm{~A}$ & 10.510 & 1.112 & 3674 & 3653 & $3663 \pm 10$ & $\mathrm{dM} 1.7 \mathrm{e}$ & $86.8570 \pm 0.1515$ & $10.204 \pm 0.024$ & $0.386 \pm 0.014$ & +0.12 & 1 & \\
\hline Gl 494B & 10.510 & 1.112 & 3674 & 3653 & $3663 \pm 10$ & $\mathrm{dM} 1.7 \mathrm{e}$ & $86.8570 \pm 0.1515$ & $10.204 \pm 0.024$ & $0.386 \pm 0.014$ & +0.12 & 1 & \\
\hline Gl 507.1 & 10.617 & 1.160 & 3626 & 3689 & $3657 \pm 32$ & $\mathrm{dM} 1.8$ & $59.3075 \pm 0.0328$ & $9.483 \pm 0.021$ & $0.542 \pm 0.034$ & +0.38 & 7 & \\
\hline Gl 508.2 & 10.66 & 1.100 & 3685 & 3591 & $3638 \pm 47$ & dM1.9 & $60.0765 \pm 0.0424$ & $9.554 \pm 0.022$ & $0.536 \pm 0.044$ & -0.31 & 9 & \\
\hline Gl 510 & 11.015 & 1.172 & 3614 & 3520 & $3567 \pm 47$ & $\mathrm{dM} 2.3$ & $64.9851 \pm 0.0534$ & $10.079 \pm 0.022$ & $0.457 \pm 0.038$ & -0.13 & 48 & \\
\hline Gl 526 & 8.464 & 1.136 & 3650 & 3580 & $3615 \pm 35$ & $\mathrm{dM} 2.0$ & $183.9836 \pm 0.0509$ & $9.788 \pm 0.021$ & $0.494 \pm 0.033$ & -0.14 & 33 & \\
\hline Gl 533.1 & 11.725 & 1.135 & 3651 & 3632 & $3642 \pm 10$ & dM1.9 & $34.9149 \pm 0.2443$ & $9.440 \pm 0.035$ & $0.562 \pm 0.024$ & & 1 & \\
\hline Gl 537A & 9.91 & 1.076 & 3707 & 3562 & $3634 \pm 72$ & dM1.9 & $89.3872 \pm 0.0661$ & $9.666 \pm 0.022$ & $0.511 \pm 0.058$ & & & \\
\hline Gl 537B & 9.91 & 1.076 & 3707 & 3562 & $3634 \pm 72$ & $\mathrm{dM} 1.9$ & $89.3822 \pm 0.0557$ & $9.666 \pm 0.021$ & $0.511 \pm 0.058$ & & & \\
\hline Gl 552 & 10.676 & 1.208 & 3579 & 3607 & $3593 \pm 14$ & SdM2.2 & $70.1774 \pm 0.0505$ & $9.907 \pm 0.022$ & $0.480 \pm 0.020$ & -0.80 & 17 & \\
\hline Gl 563 & 12.105 & 1.105 & 3681 & 3635 & $3658 \pm 23$ & $\mathrm{dM} 1.8$ & $37.0677 \pm 0.0521$ & $9.950 \pm 0.023$ & $0.436 \pm 0.023$ & -0.15 & & \\
\hline Gl $563.2 \mathrm{~A}$ & 11.656 & 1.124 & 3662 & 3665 & $3663 \pm 2$ & dM1.7 & $48.2709 \pm 0.0423$ & $10.074 \pm 0.022$ & $0.410 \pm 0.011$ & -0.36 & & \\
\hline Gl 563.2B & 12.066 & 1.196 & 3590 & 3595 & $3592 \pm 3$ & $\mathrm{dM} 2.2$ & $48.2743 \pm 0.0849$ & $10.485 \pm 0.024$ & $0.368 \pm 0.011$ & -0.36 & & \\
\hline Gl $570 \mathrm{~B}$ & 8.067 & 1.135 & 3651 & 3645 & $3648 \pm 3$ & $\mathrm{dM} 1.8$ & $170.0112 \pm 0.0851$ & $9.219 \pm 0.021$ & $0.618 \pm 0.017$ & -0.04 & 359 & Binary? \\
\hline Gl 596B & 12.400 & 1.110 & 3676 & - & $3676 \pm 30$ & dM1.6 & $23.1211 \pm 0.0409$ & $9.220 \pm 0.024$ & $0.598 \pm 0.036$ & -0.44 & & Binary? \\
\hline Gl 616.2 & 9.715 & 1.169 & 3617 & 3596 & $3607 \pm 11$ & PMS M2.1 & $49.3521 \pm 0.2170$ & $8.182 \pm 0.030$ & $1.046 \pm 0.043$ & -0.64 & 1 & PMS \\
\hline
\end{tabular}


Table 2:: continued.

\begin{tabular}{|c|c|c|c|c|c|c|c|c|c|c|c|c|}
\hline Star & $\underset{(\mathrm{mag})}{\mathrm{V}}$ & $\begin{array}{c}(\mathrm{R}-\mathrm{I})_{C} \\
(\mathrm{mag})\end{array}$ & $\begin{array}{c}T_{\text {eff }}{ }^{1} \\
(\mathrm{~K})\end{array}$ & $\begin{array}{c}T_{\text {eff }}^{2} \\
(\mathrm{~K})\end{array}$ & $T_{\text {eff }} \pm 3 \sigma^{3}$ & $\begin{array}{l}\text { Spect. } \\
\text { Type }\end{array}$ & $\begin{array}{c}\pi \\
(\mathrm{m} ")\end{array}$ & $\begin{array}{c}M_{V} \\
\text { (mag) }\end{array}$ & $\begin{array}{c}R_{\star} \\
\left(R_{\odot}\right)\end{array}$ & {$[\mathrm{M} / \mathrm{H}]$} & $\begin{array}{l}\text { Hi. Res. } \\
\text { Spectra }\end{array}$ & Comments \\
\hline Gl 618.4 & 11.783 & 1.156 & 3630 & 3606 & $3618 \pm 12$ & $\mathrm{dM} 2.0$ & $42.2851 \pm 0.0556$ & $9.914 \pm 0.023$ & $0.465 \pm 0.018$ & & ? & \\
\hline Gl 625 & 10.123 & 1.241 & 3550 & 3603 & $3576 \pm 27$ & $\mathrm{sdM} 2.3 \mathrm{e}$ & $154.4710 \pm 0.0273$ & $11.067 \pm 0.020$ & $0.287 \pm 0.016$ & -0.14 & 1 & Subdwarf? \\
\hline Gl 629.3 & 12.42 & 1.190 & 3596 & 3525 & $3560 \pm 35$ & $\mathrm{dM} 2.4$ & 46. \pm 10 & $10.734 \pm 0.447$ & $0.341 \pm 0.102$ & & 18 & Subdwarf? \\
\hline Gl 634 & 11.58 & 1.184 & 3602 & 3474 & $3538 \pm 64$ & $\mathrm{dM} 2.5$ & $63.5054 \pm 0.0638$ & $10.594 \pm 0.022$ & $0.373 \pm 0.040$ & & 31 & \\
\hline Gl 637 & 11.343 & 1.140 & 3646 & 3580 & $3613 \pm 33$ & $\mathrm{dM} 2.1$ & $63.4448 \pm 0.0290$ & $10.355 \pm 0.021$ & $0.382 \pm 0.025$ & +0.83 & 9 & \\
\hline Gl 645 & 11.44 & 1.122 & 3664 & 3703 & $3683 \pm 20$ & dM1.6 & $49.3015 \pm 0.0436$ & $9.904 \pm 0.022$ & $0.433 \pm 0.021$ & & & \\
\hline Gl $654 \mathrm{~A}$ & 10.824 & 1.172 & 3614 & 3505 & $3559 \pm 54$ & $\mathrm{dM} 2.4$ & $95.6369 \pm 0.0590$ & $10.727 \pm 0.021$ & $0.342 \pm 0.032$ & -0.38 & 123 & Subdwarf? \\
\hline Gl $654 \mathrm{~B}$ & 10.824 & 1.172 & 3614 & 3505 & $3559 \pm 54$ & $\mathrm{dM} 2.4$ & $95.6369 \pm 0.0590$ & $10.727 \pm 0.021$ & $0.342 \pm 0.032$ & -0.38 & 123 & Subdwarf? \\
\hline Gl $667 \mathrm{C}$ & 10.230 & 1.218 & 3570 & 3600 & $3585 \pm 15$ & $\mathrm{sdM} 2.2$ & $138.0171 \pm 0.0918$ & $10.930 \pm 0.021$ & $0.303 \pm 0.013$ & -0.55 & & \\
\hline Gl 672.1 & 11.635 & 1.119 & 3667 & 3590 & $3628 \pm 38$ & $\mathrm{dM} 2.0$ & $45.5127 \pm 0.0469$ & $9.926 \pm 0.022$ & $0.457 \pm 0.032$ & -0.13 & 17 & \\
\hline Gl 686 & 9.599 & 1.112 & 3674 & 3597 & $3635 \pm 38$ & dM1.9 & $122.5609 \pm 0.0346$ & $10.041 \pm 0.021$ & $0.430 \pm 0.030$ & -0.34 & 89 & \\
\hline Gl 701 & 9.367 & 1.100 & 3685 & 3664 & $3674 \pm 10$ & dM1.6 & $129.3922 \pm 0.0613$ & $9.927 \pm 0.021$ & $0.433 \pm 0.015$ & -0.31 & 117 & \\
\hline Gl 724 & 10.630 & 1.088 & 3696 & 3588 & $3642 \pm 54$ & dM1.9 & $58.9132 \pm 0.0483$ & $9.481 \pm 0.022$ & $0.552 \pm 0.050$ & +0.21 & 14 & \\
\hline Gl 737B & 10.13 & 1.094 & 3691 & 3618 & $3654 \pm 36$ & dM1.8 & $81.1583 \pm 0.0779$ & $9.677 \pm 0.022$ & $0.497 \pm 0.034$ & & & \\
\hline Gl 745A & 10.774 & 1.233 & 3557 & 3531 & $3544 \pm 13$ & $\mathrm{sdM} 2.4$ & $113.3401 \pm 0.1013$ & $11.046 \pm 0.022$ & $0.301 \pm 0.012$ & -0.31 & 1 & Subdwarf? \\
\hline Gl 745B & 10.771 & 1.219 & 3569 & 3531 & $3550 \pm 19$ & sdM2.4 & $113.2063 \pm 0.0460$ & $11.040 \pm 0.021$ & $0.300 \pm 0.014$ & -0.31 & 1 & Subdwarf? \\
\hline Gl 781 & 11.975 & 1.064 & 3718 & 3566 & $3642 \pm 76$ & $\mathrm{sdM} 1.9 \mathrm{e}$ & $60.3913 \pm 0.0343$ & $10.880 \pm 0.021$ & $0.290 \pm 0.035$ & -0.88 & & \\
\hline Gl $800 \mathrm{~A}$ & 10.735 & 1.100 & 3685 & 3590 & $3637 \pm 47$ & dM1.9 & $52.4920 \pm 0.0449$ & $9.335 \pm 0.022$ & $0.594 \pm 0.049$ & -0.06 & 9 & \\
\hline Gl 803 & 8.794 & 1.100 & 3685 & 3673 & $3679 \pm 6$ & $\mathrm{dM} 1.6 \mathrm{e}$ & $102.8295 \pm 0.0486$ & $8.855 \pm 0.021$ & $0.705 \pm 0.021$ & +0.10 & 24 & PMS \\
\hline Gl 806 & 10.79 & 1.199 & 3587 & 3565 & $3576 \pm 11$ & $\mathrm{dM} 2.3$ & $82.9968 \pm 0.0296$ & $10.385 \pm 0.021$ & $0.393 \pm 0.015$ & -0.12 & 40 & \\
\hline Gl 808 & 11.86 & 1.184 & 3602 & 3448 & $3525 \pm 77$ & $\mathrm{dM} 2.6$ & $58.8020 \pm 0.0295$ & $10.707 \pm 0.021$ & $0.360 \pm 0.045$ & & 4 & Subdwarf? \\
\hline Gl $812 \mathrm{~A}$ & 11.893 & 1.140 & 3646 & 3399 & $3522 \pm 123$ & dM2.6 & $62.2541 \pm 0.0958$ & $10.864 \pm 0.023$ & $0.336 \pm 0.064$ & +0.12 & & Subdwarf? \\
\hline Gl 813 & 12.013 & 1.231 & 3559 & 3455 & $3507 \pm 52$ & sdM2.7 & $69.3112 \pm 0.0676$ & $11.217 \pm 0.022$ & $0.291 \pm 0.027$ & -0.32 & 1 & Subdwarf? \\
\hline Gl $815 \mathrm{~A}$ & 10.798 & 1.184 & 3602 & 3508 & $3555 \pm 47$ & $\mathrm{dM} 2.4 \mathrm{e}$ & $66.8056 \pm 0.0926$ & $9.922 \pm 0.023$ & $0.499 \pm 0.042$ & -0.20 & 1 & \\
\hline Gl 815B & 10.798 & 1.184 & 3602 & 3508 & $3555 \pm 47$ & $\mathrm{dM} 2.4 \mathrm{e}$ & $66.8056 \pm 0.0926$ & $9.922 \pm 0.023$ & $0.499 \pm 0.042$ & -0.20 & 1 & \\
\hline Gl 821 & 10.869 & 1.116 & 3670 & 3559 & $3614 \pm 55$ & $\mathrm{dM} 2.1$ & $84.7044 \pm 0.0685$ & $10.509 \pm 0.022$ & $0.355 \pm 0.033$ & -0.45 & 107 & Subdwarf? \\
\hline Gl 832 & 8.663 & 1.196 & 3590 & 3475 & $3532 \pm 57$ & $\mathrm{dM} 2.5$ & $201.4073 \pm 0.0429$ & $10.183 \pm 0.020$ & $0.454 \pm 0.018$ & -0.24 & 66 & \\
\hline Gl 838.6 & 12.002 & 1.237 & 3553 & - & $3553 \pm 30$ & $\mathrm{dM} 2.4$ & $64.3588 \pm 0.0351$ & $11.045 \pm 0.021$ & $0.298 \pm 0.020$ & & & Subdwarf? \\
\hline Gl 842 & 9.737 & 1.039 & 3741 & 3613 & $3677 \pm 64$ & dM1.6 & $86.5180 \pm 0.0368$ & $9.423 \pm 0.021$ & $0.544 \pm 0.056$ & & 23 & \\
\hline Gl 851 & 10.221 & 1.237 & 3553 & & $3553 \pm 30$ & $\mathrm{dM} 2.4$ & $86.2883 \pm 0.0433$ & $9.901 \pm 0.021$ & $0.505 \pm 0.031$ & & & \\
\hline Gl 865 & 11.483 & 1.170 & 3616 & 3396 & $3506 \pm 110$ & $\mathrm{dM} 2.7$ & $75.87 \pm 5.83$ & $10.883 \pm 0.181$ & $0.339 \pm 0.088$ & -0.10 & & Subdwarf? \\
\hline Gl $867 \mathrm{~A}$ & 9.079 & 1.196 & 3590 & 3658 & $3623 \pm 35$ & $\mathrm{dM} 2.0 \mathrm{e}$ & $112.3669 \pm 0.0834$ & $9.332 \pm 0.022$ & $0.604 \pm 0.040$ & & 2 & Binary? \\
\hline Gl 867BA & 12.18 & 1.196 & 3590 & 3658 & $3623 \pm 35$ & $\mathrm{dM} 2.0 \mathrm{e}$ & $113.1171 \pm 0.0728$ & $12.448 \pm 0.021$ & $0.144 \pm 0.010$ & & 3 & \\
\hline Gl $867 \mathrm{BB}$ & 12.18 & 1.196 & 3590 & 3658 & $3623 \pm 35$ & $\mathrm{dM} 2.0 \mathrm{e}$ & $113.1171 \pm 0.0728$ & $12.448 \pm 0.021$ & $0.144 \pm 0.010$ & & 3 & \\
\hline Gl 880 & 8.657 & 1.125 & 3661 & 3614 & $3622 \pm 8$ & $\mathrm{dM} 2.0$ & $145.6107 \pm 0.0388$ & $9.473 \pm 0.021$ & $0.567 \pm 0.019$ & & 110 & \\
\hline Gl 887 & 7.351 & 1.088 & 3696 & 3628 & $3662 \pm 34$ & dM1.7 & $304.2190 \pm 0.0451$ & $9.767 \pm 0.020$ & $0.473 \pm 0.030$ & & 88 & \\
\hline Gl 895 & 10.033 & 1.117 & 3669 & 3656 & $3662 \pm 6$ & dM1.7 & $75.5654 \pm 0.0333$ & $9.425 \pm 0.021$ & $0.553 \pm 0.017$ & & 3 & \\
\hline Gl 901 & 13.105 & 1.157 & 3629 & - & $3629 \pm 30$ & sdM2.0 & $40.3336 \pm 0.0518$ & $11.133 \pm 0.023$ & $0.262 \pm 0.016$ & & 1 & Subdwarf? \\
\hline Gl 908 & 8.993 & 1.117 & 3669 & 3640 & $3654 \pm 14$ & sdM1.8 & $169.3585 \pm 0.0595$ & $10.137 \pm 0.021$ & $0.402 \pm 0.016$ & -0.57 & 122 & \\
\hline STKM 1-265 & 12.220 & 1.186 & 3600 & 3717 & $3659 \pm 59$ & $\mathrm{dM} 1.7$ & $28.2136 \pm 0.0631$ & $9.472 \pm 0.025$ & $0.543 \pm 0.053$ & +0.25 & 2 & \\
\hline STKM 1-474 & 10.385 & 1.135 & 3651 & 3633 & $3642 \pm 9$ & M1.9III & $1.3616 \pm 0.0646$ & $1.055 \pm 0.121$ & $26.727 \pm 2.209$ & & 1 & \\
\hline STKM 1-555 & 10.975 & 1.110 & 3676 & 3679 & $3678 \pm 2$ & M1.6III & $0.8524 \pm 0.0362$ & $0.628 \pm 0.110$ & $31.207 \pm 2.129$ & & 1 & \\
\hline STKM 1-633 & 12.444 & 1.148 & 3638 & - & $3638 \pm 30$ & dM1.9 & $23.1904 \pm 0.0631$ & $9.271 \pm 0.026$ & $0.611 \pm 0.038$ & & & Binary? \\
\hline STKM 1-724B & 12.23 & 1.123 & 3663 & - & $3663 \pm 30$ & sdM1.7 & $25.7756 \pm 0.0514$ & $9.286 \pm 0.024$ & $0.589 \pm 0.036$ & & & \\
\hline STKM $1-828$ & 12.783 & 1.161 & 3625 & 3650 & $3638 \pm 12$ & $\mathrm{dM} 1.9$ & $33.8184 \pm 0.0911$ & $10.429 \pm 0.026$ & $0.358 \pm 0.014$ & -1.05 & & \\
\hline STKM 1-873 & 12.46 & 1.148 & 3638 & 3537 & $3588 \pm 50$ & $\mathrm{dM} 2.2$ & $36.3423 \pm 0.0439$ & $10.262 \pm 0.023$ & $0.410 \pm 0.036$ & -0.24 & & \\
\hline STKM 1-928 & 10.85 & 1.136 & 3650 & 3713 & $3681 \pm 62$ & $\mathrm{dM} 1.6$ & $59.9389 \pm 0.0527$ & $9.739 \pm 0.022$ & $0.468 \pm 0.047$ & & 2 & \\
\hline STKM 1-1264 & 12.315 & 1.148 & 3638 & 3638 & $3638 \pm 1$ & dM1.9 & $19.0322 \pm 0.1398$ & $8.712 \pm 0.036$ & $0.790 \pm 0.025$ & +0.14 & & Binary? \\
\hline HIP 263 & 12.037 & 1.110 & 3676 & 3655 & $3665 \pm 10$ & dM1.7 & $34.8918 \pm 0.0927$ & $9.751 \pm 0.026$ & $0.475 \pm 0.018$ & -0.19 & & \\
\hline HIP 15401 & 12.279 & 1.097 & 3688 & 3626 & $3657 \pm 31$ & dM1.8 & $28.3222 \pm 0.0524$ & $9.540 \pm 0.024$ & $0.528 \pm 0.033$ & -0.25 & 1 & \\
\hline HIP 39402 & 11.358 & 1.148 & 3638 & 3670 & $3654 \pm 16$ & dM1.8 & $31.87 \pm 3.39$ & $8.875 \pm 0.240$ & $0.719 \pm 0.110$ & & 1 & Binary? \\
\hline HIP 48509 & 12.023 & 1.123 & 3663 & 3617 & $3640 \pm 23$ & dM1.9 & $39.6039 \pm 0.0327$ & $10.012 \pm 0.022$ & $0.433 \pm 0.022$ & & & \\
\hline HIP 117461 & 12.609 & 1.098 & 3687 & 3581 & $3634 \pm 53$ & dM1.9 & $21.0791 \pm 0.0475$ & $9.228 \pm 0.025$ & $0.626 \pm 0.057$ & -0.06 & & \\
\hline
\end{tabular}


Table 2:: continued.

\begin{tabular}{|c|c|c|c|c|c|c|c|c|c|c|c|c|}
\hline Star & $\underset{(\mathrm{mag})}{\mathrm{V}}$ & $\begin{array}{c}(\mathrm{R}-\mathrm{I})_{C} \\
(\mathrm{mag})\end{array}$ & $\begin{array}{c}T_{e f f}{ }^{1} \\
(\mathrm{~K})\end{array}$ & $\begin{array}{c}T_{e f f}^{2} \\
(\mathrm{~K})\end{array}$ & $\begin{array}{c}T_{\text {eff }} \pm 3 \sigma^{3} \\
(\mathrm{~K})\end{array}$ & $\begin{array}{l}\text { Spect. } \\
\text { Type }\end{array}$ & $\begin{array}{c}\pi \\
(\mathrm{m} ")\end{array}$ & $\begin{array}{c}M_{V} \\
(\mathrm{mag})\end{array}$ & $\begin{array}{c}R_{\star} \\
\left(R_{\odot}\right)\end{array}$ & {$[\mathrm{M} / \mathrm{H}]$} & $\begin{array}{l}\text { Hi. Res. } \\
\text { Spectra }\end{array}$ & Comments \\
\hline VB 12 & 16.46 & 1.12 & 3665 & 3500 & $3582 \pm 82$ & sdM3 & $15.8988 \pm 0.0634$ & $12.467 \pm 0.029$ & $0.150 \pm 0.020$ & -1.43 & 3 & \\
\hline LP 97-674A & 12.479 & 1.110 & 3676 & 3687 & $3682 \pm 6$ & $\mathrm{dM} 1.6$ & $35.1609 \pm 0.0277$ & $10.209 \pm 0.022$ & $0.377 \pm 0.011$ & -0.32 & & \\
\hline LP 97-674B & 12.479 & 1.110 & 3676 & 3687 & $3682 \pm 6$ & $\mathrm{dM} 1.6$ & $35.2634 \pm 0.0283$ & $10.216 \pm 0.022$ & $0.376 \pm 0.011$ & -0.32 & & \\
\hline LP $300-80$ & 13.86 & 1.199 & 3587 & - & $3587 \pm 30$ & $\mathrm{dM} 2.2$ & $16.8188 \pm 0.1325$ & $9.989 \pm 0.037$ & $0.466 \pm 0.032$ & & & \\
\hline LP $333-29$ & 12.538 & 1.251 & 3541 & 3509 & $3525 \pm 16$ & sdM2.6 & $35.3210 \pm 0.0381$ & $10.278 \pm 0.022$ & $0.438 \pm 0.020$ & -0.67 & & \\
\hline LP $381-40$ & 13.08 & 1.186 & 3600 & - & $3600 \pm 30$ & $\mathrm{dM} 2.1$ & $19.6454 \pm 0.0585$ & $9.546 \pm 0.026$ & $0.562 \pm 0.036$ & & & \\
\hline LP 494-80 & 13.79 & 1.135 & 3651 & - & $3651 \pm 30$ & $\mathrm{dM} 1.8$ & $12.5123 \pm 0.0453$ & $9.277 \pm 0.028$ & $0.600 \pm 0.038$ & & & \\
\hline LP 553-74 & 14.13 & 1.237 & 3553 & - & $3553 \pm 30$ & dM2.4 & $15.8499 \pm 0.0394$ & $10.130 \pm 0.025$ & $0.454 \pm 0.029$ & & & \\
\hline LHS 272 & 13.161 & 1.246 & 3546 & 3500 & $3523 \pm 23$ & sdM2.6 & $74.5686 \pm 0.0440$ & $12.524 \pm 0.021$ & $0.156 \pm 0.008$ & & & Subdwarf? \\
\hline LHS 276 & 11.90 & 1.044 & 3736 & 3587 & $3662 \pm 74$ & dM1.7 & $36.4522 \pm 0.0793$ & $9.709 \pm 0.025$ & $0.486 \pm 0.057$ & -0.97 & & \\
\hline LHS 1155 & 12.65 & 1.196 & 3590 & 3413 & $3501 \pm 88$ & dM2.7 & $36.4386 \pm 0.0281$ & $10.458 \pm 0.022$ & $0.415 \pm 0.058$ & & $?$ & \\
\hline LHS 1353 & 12.72 & 1.193 & 3593 & 3545 & $3569 \pm 24$ & $\operatorname{sdM} 2.3$ & $59.8319 \pm 0.0790$ & $11.605 \pm 0.023$ & $0.226 \pm 0.012$ & -0.27 & & Subdwarf? \\
\hline LHS 1354 & 15.05 & 1.193 & 3593 & 3545 & $3569 \pm 24$ & sdM? & $59.8828 \pm 0.0509$ & $13.937 \pm 0.022$ & $0.077 \pm 0.004$ & -0.27 & & \\
\hline LHS 1380 & 11.638 & 1.059 & 3723 & 3579 & $3651 \pm 72$ & $\mathrm{dM} 1.8$ & $36.5391 \pm 0.0338$ & $9.452 \pm 0.022$ & $0.553 \pm 0.063$ & & & \\
\hline LHS 1566 & 13.810 & 1.224 & 3565 & - & $3565 \pm 30$ & $\operatorname{sdM} 2.3$ & $28.5046 \pm 0.0752$ & $11.085 \pm 0.026$ & $0.288 \pm 0.018$ & -0.11 & & Subdwarf? \\
\hline LHS 1854 & 15.03 & 1.199 & 3587 & - & $3587 \pm 30$ & dM2.2 & - & \pm & \pm & & & \\
\hline LHS 1871 & 13.95 & 1.151 & 3635 & - & $3635 \pm 30$ & sdM1.9 & $18.7798 \pm 0.1022$ & $10.318 \pm 0.032$ & $0.378 \pm 0.025$ & & & \\
\hline LHS 1907 & 14.515 & 1.110 & 3676 & - & $3676 \pm 30$ & dM1.6 & $12.1798 \pm 0.0456$ & $9.943 \pm 0.028$ & $0.429 \pm 0.027$ & & & \\
\hline LHS 2121 & 12.274 & 1.172 & 3614 & 3627 & $3621 \pm 7$ & $\mathrm{dM} 2.0$ & $30.4160 \pm 0.1247$ & $9.690 \pm 0.029$ & $0.514 \pm 0.018$ & -0.12 & & \\
\hline LHS 2129 & 13.41 & 1.202 & 3584 & - & $3584 \pm 30$ & $\mathrm{dM} 2.2$ & $28.2727 \pm 0.0471$ & $10.667 \pm 0.024$ & $0.342 \pm 0.021$ & & & Subdwarf? \\
\hline LHS 2238 & 13.63 & 1.186 & 3600 & - & $3600 \pm 30$ & $\mathrm{sdM} 2.1$ & $31.7196 \pm 0.1161$ & $11.137 \pm 0.028$ & $0.270 \pm 0.017$ & & & Subdwarf? \\
\hline LHS 2383 & 12.773 & 1.170 & 3616 & - & $3616 \pm 30$ & sdM2.1 & $42.6258 \pm 0.0501$ & $10.921 \pm 0.023$ & $0.293 \pm 0.018$ & & & Subdwarf? \\
\hline LHS 2420 & 12.475 & 1.237 & 3553 & 3557 & $3555 \pm 3$ & dM2.4 & $35.4805 \pm 0.0563$ & $10.225 \pm 0.023$ & $0.434 \pm 0.013$ & -0.31 & & \\
\hline LHS 2439 & 11.92 & 1.174 & 3612 & 3578 & $3595 \pm 17$ & $\mathrm{dM} 2.2$ & $35.6955 \pm 0.0294$ & $9.683 \pm 0.022$ & $0.531 \pm 0.024$ & +0.58 & & \\
\hline LHS 2518 & 13.262 & 1.233 & 3557 & - & $3557 \pm 30$ & $\mathrm{dM} 2.4$ & $27.7727 \pm 0.0542$ & $10.480 \pm 0.024$ & $0.385 \pm 0.024$ & & & Subdwarf? \\
\hline LHS 3050 & 14.770 & 1.148 & 3638 & - & $3638 \pm 30$ & sdM1.9 & $28.7223 \pm 0.0611$ & $12.061 \pm 0.025$ & $0.169 \pm 0.010$ & & & Subdwarf? \\
\hline LHS 3141 & 14.259 & 1.211 & 3576 & - & $3576 \pm 30$ & sdM2.3 & $26.4396 \pm 0.1935$ & $11.370 \pm 0.036$ & $0.250 \pm 0.017$ & +0.16 & & Subdwarf? \\
\hline LHS 3258A & 12.671 & 1.110 & 3676 & 3648 & $3662 \pm 14$ & sdM1.7 & $37.1037 \pm 0.0266$ & $10.518 \pm 0.022$ & $0.334 \pm 0.013$ & & 1 & Subdwarf? \\
\hline LHS $3258 \mathrm{~B}$ & 12.671 & 1.110 & 3676 & 3648 & $3662 \pm 14$ & sdM1.7 & $37.1037 \pm 0.0266$ & $10.518 \pm 0.022$ & $0.334 \pm 0.013$ & & 1 & Subdwarf? \\
\hline LHS 3334 & 15.050 & 1.194 & 3592 & - & $3592 \pm 30$ & $\operatorname{sdM} 2.2$ & $17.5697 \pm 0.0258$ & $11.274 \pm 0.023$ & $0.256 \pm 0.016$ & & & Subdwarf? \\
\hline LHS 3402 & 11.585 & 1.110 & 3676 & 3689 & $3683 \pm 6$ & dM1.6 & $41.7891 \pm 0.0328$ & $9.690 \pm 0.022$ & $0.478 \pm 0.014$ & -0.47 & 1 & \\
\hline LHS 3621 & 12.810 & 1.134 & 3652 & - & $3652 \pm 30$ & $\mathrm{dM} 1.8$ & $25.0913 \pm 0.0355$ & $9.808 \pm 0.023$ & $0.469 \pm 0.029$ & & & \\
\hline LDS $3000 B$ & 13.91 & 1.212 & 3575 & - & $3575 \pm 30$ & $\mathrm{dM} 2.3$ & $20.7130 \pm 0.0706$ & $10.491 \pm 0.027$ & $0.375 \pm 0.024$ & & & Subdwarf? \\
\hline LDS $4900 B$ & 14.64 & 1.199 & 3587 & - & $3587 \pm 30$ & $\mathrm{dM} 2.2$ & $12.0217 \pm 0.0344$ & $10.040 \pm 0.026$ & $0.455 \pm 0.029$ & & & \\
\hline G $43-43$ & 12.712 & 1.135 & 3651 & - & $3651 \pm 30$ & $\mathrm{dM} 1.8$ & $27.5395 \pm 0.0688$ & $9.912 \pm 0.025$ & $0.448 \pm 0.028$ & -0.01 & & \\
\hline G $74-41$ & 14.345 & 1.224 & 3565 & - & $3565 \pm 30$ & sdM2.3 & $17.8732 \pm 0.0353$ & $10.606 \pm 0.024$ & $0.360 \pm 0.023$ & -0.42 & & \\
\hline G $72-38 \mathrm{~A}$ & 13.260 & 1.237 & 3553 & - & $3553 \pm 30$ & $\mathrm{dM} 2.4$ & $28.0507 \pm 0.0505$ & $10.500 \pm 0.024$ & $0.383 \pm 0.024$ & & 1 & \\
\hline G $72-38 B$ & 13.260 & 1.237 & 3553 & - & $3553 \pm 30$ & $\mathrm{dM} 2.4$ & $28.0507 \pm 0.0505$ & $10.500 \pm 0.024$ & $0.383 \pm 0.024$ & & 1 & \\
\hline G $79-16$ & 13.067 & 1.174 & 3612 & - & $3612 \pm 30$ & $\mathrm{dM} 2.1$ & $18.7989 \pm 0.0487$ & $9.438 \pm 0.026$ & $0.583 \pm 0.037$ & & 1 & \\
\hline G $127-106$ & 15.72 & 1.155 & 3631 & - & $3631 \pm 30$ & $\operatorname{sdM} 2.0$ & $13.7239 \pm 0.0401$ & $11.407 \pm 0.026$ & $0.230 \pm 0.014$ & & & Subdwarf? \\
\hline G $137-18$ & 13.57 & 1.224 & 3565 & - & $3565 \pm 30$ & $\mathrm{dM} 2.3$ & $17.5917 \pm 0.0487$ & $9.797 \pm 0.026$ & $0.522 \pm 0.033$ & & & \\
\hline G $172-35$ & 12.74 & 1.199 & 3587 & - & $3587 \pm 30$ & $\mathrm{dM} 2.2$ & $33.6078 \pm 0.0451$ & $10.372 \pm 0.023$ & $0.390 \pm 0.024$ & & 1 & \\
\hline G $217-40$ & 14.39 & 1.237 & 3553 & - & $3553 \pm 30$ & $\mathrm{dM} 2.4$ & $13.0369 \pm 0.0475$ & $9.966 \pm 0.028$ & $0.490 \pm 0.032$ & +0.22 & & \\
\hline G $236-68$ & 12.938 & 1.110 & 3676 & - & $3676 \pm 30$ & $\mathrm{dM} 1.6$ & $18.3960 \pm 0.0302$ & $9.262 \pm 0.024$ & $0.587 \pm 0.036$ & & & \\
\hline G 265-9 & 13.575 & 1.135 & 3651 & - & $3651 \pm 30$ & dM1.8 & $17.2024 \pm 0.0461$ & $9.753 \pm 0.026$ & $0.482 \pm 0.030$ & & & \\
\hline $\mathrm{BD}+294630$ & 10.660 & 1.072 & 3711 & 4519 & $4519 \pm 30$ & K3.9III & $0.9909 \pm 0.0372$ & $0.640 \pm 0.100$ & $14.129 \pm 1.100$ & & 2 & \\
\hline
\end{tabular}


Table 2:: continued.

\begin{tabular}{|c|c|c|c|c|c|c|c|c|c|c|c|c|}
\hline Star & $\begin{array}{c}\mathrm{V} \\
(\mathrm{mag})\end{array}$ & $\begin{array}{c}(\mathrm{R}-\mathrm{I})_{C} \\
(\mathrm{mag})\end{array}$ & $\begin{array}{c}T_{e f f}{ }^{1} \\
(\mathrm{~K})\end{array}$ & $\begin{array}{c}T_{e f f}^{2} \\
(\mathrm{~K})\end{array}$ & $T_{(\mathrm{K})}^{T_{\text {eff }} \pm 3 \sigma^{3}}$ & $\begin{array}{l}\text { Spect. } \\
\text { Type }\end{array}$ & $\begin{array}{c}\pi \\
(\mathrm{m} ")\end{array}$ & $\begin{array}{c}M_{V} \\
\text { (mag) }\end{array}$ & $\begin{array}{c}R_{\star} \\
\left(R_{\odot}\right)\end{array}$ & {$[\mathrm{M} / \mathrm{H}]$} & $\begin{array}{l}\text { Hi. Res. } \\
\text { Spectra }\end{array}$ & Comments \\
\hline GJ 1012 & 12.23 & 1.462 & 3378 & - & $3378 \pm 26$ & $\mathrm{dM} 3.4$ & $74.7749 \pm 0.0995$ & $11.599 \pm 0.023$ & $0.308 \pm 0.025$ & -0.11 & & \\
\hline GJ 1013 & 12.745 & 1.462 & 3378 & 3243 & $3311 \pm 68$ & $\mathrm{dM} 3.7$ & $70.9713 \pm 0.0530$ & $12.000 \pm 0.022$ & $0.290 \pm 0.051$ & -0.07 & & \\
\hline GJ 1022 & 12.18 & 1.262 & 3532 & 3525 & $3529 \pm 4$ & $\mathrm{dM} 2.6$ & $51.2584 \pm 0.0410$ & $10.729 \pm 0.022$ & $0.355 \pm 0.011$ & -0.47 & & \\
\hline GJ $1026 \mathrm{~A}$ & 12.05 & 1.322 & 3485 & 3391 & $3438 \pm 47$ & dM3.1 & $62.1000 \pm 0.2033$ & $11.015 \pm 0.027$ & $0.360 \pm 0.045$ & & & \\
\hline GJ $1026 \mathrm{~B}$ & 11.35 & 1.322 & 3485 & 3553 & $3519 \pm 34$ & $\mathrm{dM} 2.6$ & $62.6270 \pm 0.0425$ & $10.334 \pm 0.021$ & $0.430 \pm 0.029$ & & 1 & \\
\hline GJ 1046 & 11.595 & 1.292 & 3506 & 3507 & $3506 \pm 1$ & $\mathrm{dM} 2.7$ & $65.8012 \pm 0.1222$ & $10.686 \pm 0.024$ & $0.371 \pm 0.010$ & & 36 & \\
\hline GJ 1050 & 11.730 & 1.322 & 3484 & 3357 & $3420 \pm 64$ & $\mathrm{sdM} 3.2$ & $91.9958 \pm 0.0393$ & $11.549 \pm 0.021$ & $0.291 \pm 0.046$ & -1.20 & 1 & \\
\hline GJ 1054B & 13.095 & 1.363 & 3456 & - & $3456 \pm 26$ & $\mathrm{dM} 3.0 \mathrm{e}$ & $51.0297 \pm 0.0401$ & $11.634 \pm 0.022$ & $0.262 \pm 0.020$ & -0.09 & & \\
\hline GJ 1065 & 12.817 & 1.475 & 3366 & 3241 & $3304 \pm 62$ & $\mathrm{dM} 3.8$ & $100.5599 \pm 0.1050$ & $12.829 \pm 0.022$ & $0.201 \pm 0.033$ & +0.20 & & \\
\hline GJ $1077 \mathrm{~A}$ & 12.66 & 1.38 & 3444 & 3559 & $3501 \pm 58$ & sdM2.7 & $62.8435 \pm 0.0376$ & $11.654 \pm 0.021$ & $0.239 \pm 0.021$ & -0.64 & 9 & \\
\hline GJ $1077 \mathrm{~B}$ & 12.66 & 1.38 & 3444 & 3559 & $3501 \pm 58$ & sdM2.7 & $62.8435 \pm 0.0376$ & $11.654 \pm 0.021$ & $0.239 \pm 0.021$ & -0.64 & 9 & \\
\hline GJ 1080 & 12.835 & 1.344 & 3469 & 3367 & $3418 \pm 51$ & $\mathrm{dM} 3.2$ & $47.0904 \pm 0.1426$ & $11.200 \pm 0.027$ & $0.343 \pm 0.046$ & -0.44 & 1 & \\
\hline GJ 1081 & 12.232 & 1.475 & 3366 & 3449 & $3408 \pm 41$ & $\mathrm{dM} 3.2$ & $65.4374 \pm 1.0182$ & $11.311 \pm 0.054$ & $0.332 \pm 0.042$ & & 1 & \\
\hline GJ 1097 & 11.456 & 1.411 & 3422 & 3448 & $3435 \pm 13$ & $\mathrm{dM} 3.1$ & $85.3378 \pm 0.0694$ & $11.112 \pm 0.022$ & $0.347 \pm 0.018$ & -0.04 & 4 & \\
\hline GJ 1099 & 11.93 & 1.315 & 3489 & 3464 & $3476 \pm 12$ & sdM2.9 & $64.0879 \pm 0.0634$ & $10.964 \pm 0.022$ & $0.344 \pm 0.017$ & -0.57 & 8 & \\
\hline GJ $1108 \mathrm{~B}$ & 12.12 & 1.487 & 3356 & - & $3356 \pm 26$ & $\mathrm{dM} 3.5$ & $40.7671 \pm 0.1068$ & $10.923 \pm 0.026$ & $0.437 \pm 0.029$ & -0.02 & 1 & \\
\hline GJ $1108 \mathrm{C}$ & 12.12 & 1.487 & 3356 & - & $3356 \pm 26$ & $\mathrm{dM} 3.5$ & $40.7671 \pm 0.1068$ & $10.923 \pm 0.026$ & $0.437 \pm 0.029$ & -0.02 & 1 & \\
\hline GJ $1110 \mathrm{~A}$ & 13.848 & 1.324 & 3482 & 3550 & $3516 \pm 34$ & $\mathrm{sdM} 2.6$ & $42.5844 \pm 0.0746$ & $11.994 \pm 0.024$ & $0.201 \pm 0.011$ & -0.71 & & \\
\hline GJ 1110B & 13.848 & 1.324 & 3482 & 3550 & $3516 \pm 34$ & sdM2.6 & $42.5844 \pm 0.0746$ & $11.994 \pm 0.024$ & $0.201 \pm 0.011$ & -0.71 & & \\
\hline GJ 1125 & 11.710 & 1.125 & 3661 & 3280 & $3470 \pm 190$ & $\mathrm{sdM} 2.9$ & $100.9992 \pm 0.0746$ & $11.732 \pm 0.022$ & $0.244 \pm 0.114$ & -0.14 & 5 & Subdwarf? \\
\hline GJ 1146 & 13.57 & 1.388 & 3439 & - & $3439 \pm 26$ & sdM3.1 & $50.2385 \pm 0.0654$ & $12.075 \pm 0.023$ & $0.221 \pm 0.017$ & -0.56 & & \\
\hline GJ 1168 & 12.945 & 1.481 & 3361 & 3298 & $3330 \pm 32$ & $\mathrm{dM} 3.6$ & $45.5233 \pm 0.0660$ & $11.236 \pm 0.023$ & $0.398 \pm 0.038$ & -0.50 & & \\
\hline GJ 1169 & 13.163 & 1.475 & 3366 & - & $3366 \pm 26$ & dM3.4 & $64.8326 \pm 0.0507$ & $12.222 \pm 0.022$ & $0.236 \pm 0.019$ & -0.48 & & Subdwarf? \\
\hline GJ 1185 & 13.296 & 1.277 & 3519 & - & $3519 \pm 26$ & $\mathrm{dM} 2.6$ & $54.8984 \pm 0.0663$ & $11.994 \pm 0.023$ & $0.200 \pm 0.012$ & +0.01 & & Subdwarf? \\
\hline GJ 1197 & 13.25 & 1.304 & 3496 & 3535 & $3516 \pm 19$ & $\mathrm{dM} 2.6$ & $44.3889 \pm 0.0355$ & $11.486 \pm 0.022$ & $0.254 \pm 0.012$ & -0.12 & & \\
\hline GJ $1200 \mathrm{~A}$ & 13.68 & 1.419 & 3415 & 3403 & $3409 \pm 6$ & sdM3.2 & $58.9533 \pm 0.1137$ & $12.536 \pm 0.024$ & $0.189 \pm 0.005$ & -0.49 & & Subdwarf? \\
\hline GJ $1200 \mathrm{~B}$ & 13.68 & 1.419 & 3415 & 3403 & $3409 \pm 6$ & $\mathrm{sdM} 3.2$ & $58.9533 \pm 0.1137$ & $12.536 \pm 0.024$ & $0.189 \pm 0.005$ & -0.49 & & Subdwarf? \\
\hline GJ 1202 & 12.76 & 1.401 & 3430 & 3393 & $3411 \pm 18$ & $\mathrm{dM} 3.2$ & $59.4685 \pm 0.0922$ & $11.631 \pm 0.023$ & $0.285 \pm 0.018$ & -0.24 & & \\
\hline GJ 1203 & 12.158 & 1.352 & 3463 & 3515 & $3489 \pm 26$ & $\mathrm{dM} 2.8$ & $54.0491 \pm 0.1455$ & $10.822 \pm 0.026$ & $0.359 \pm 0.028$ & & 2 & \\
\hline GJ 1209 & 12.25 & 1.300 & 3499 & 3483 & $3491 \pm 8$ & $\mathrm{dM} 2.8$ & $60.8288 \pm 0.0607$ & $11.171 \pm 0.022$ & $0.304 \pm 0.013$ & -0.33 & & \\
\hline GJ $1212 \mathrm{~A}$ & 12.785 & 1.403 & 3428 & 3396 & $3412 \pm 16$ & $\mathrm{dM} 3.2$ & $50.0345 \pm 0.3128$ & $11.281 \pm 0.034$ & $0.334 \pm 0.022$ & & 2 & \\
\hline GJ $1212 B$ & 12.785 & 1.403 & 3428 & 3396 & $3412 \pm 16$ & $\mathrm{dM} 3.2$ & $50.0345 \pm 0.3128$ & $11.281 \pm 0.034$ & $0.334 \pm 0.022$ & & 2 & \\
\hline GJ 1226 & 12.71 & 1.450 & 3388 & 3227 & $3308 \pm 81$ & $\mathrm{dM} 3.7$ & $62 \pm 15$ & $11.672 \pm 0.490$ & $0.339 \pm 0.169$ & -0.07 & & \\
\hline GJ 1236 & 12.388 & 1.433 & 3403 & 3329 & $3366 \pm 37$ & dM3.4 & $94.1932 \pm 0.0657$ & $12.258 \pm 0.022$ & $0.232 \pm 0.024$ & -0.27 & & Subdwarf? \\
\hline GJ 1248 & 12.162 & 1.250 & 3542 & 3436 & $3489 \pm 53$ & $\mathrm{dM} 2.8$ & $75.3873 \pm 0.0597$ & $11.548 \pm 0.022$ & $0.257 \pm 0.034$ & -0.34 & & Subdwarf? \\
\hline GJ 1271 & 11.704 & 1.298 & 3500 & 3549 & $3524 \pm 25$ & $\mathrm{dM} 2.6$ & $47.52 \pm 2.82$ & $10.088 \pm 0.145$ & $0.479 \pm 0.056$ & +0.15 & 9 & \\
\hline GJ 1292 & 11.72 & 1.425 & 3410 & 3411 & $3410 \pm 1$ & $\mathrm{dM} 3.7$ & $74.0641 \pm 1.0281$ & $11.068 \pm 0.050$ & $0.370 \pm 0.016$ & +0.89 & 5 & \\
\hline GJ 2121 & 12.280 & 1.386 & 3440 & 3456 & $3448 \pm 8$ & dM3.0 & $45.9023 \pm 0.2469$ & $10.589 \pm 0.032$ & $0.430 \pm 0.020$ & +0.14 & 28 & \\
\hline GJ $2155 \mathrm{~A}$ & 11.317 & 1.269 & 3526 & 3863 & $3695 \pm 168$ & $\mathrm{dM} 1.5$ & $44.3862 \pm 0.0696$ & $9.553 \pm 0.023$ & $0.502 \pm 0.117$ & +0.30 & 1 & PMS? \\
\hline GJ $2155 \mathrm{~B}$ & 11.317 & 1.269 & 3526 & 3863 & $3695 \pm 168$ & $\mathrm{dM} 1.5$ & $44.3862 \pm 0.0696$ & $9.553 \pm 0.023$ & $0.502 \pm 0.117$ & +0.30 & 1 & PMS? \\
\hline GJ 3006 & 12.162 & 1.320 & 3485 & 3445 & $3465 \pm 20$ & $\mathrm{dM} 2.9$ & $57.3441 \pm 0.0663$ & $10.954 \pm 0.023$ & $0.353 \pm 0.023$ & & & \\
\hline GJ 3007 & 13.063 & 1.376 & 3447 & 3459 & $3453 \pm 6$ & sdM3.0 & $35.9604 \pm 0.1136$ & $10.842 \pm 0.027$ & $0.380 \pm 0.015$ & -0.79 & & \\
\hline GJ 3032 & 11.887 & 1.283 & 3513 & 3458 & $3486 \pm 28$ & $\mathrm{dM} 2.8$ & $49.6322 \pm 0.0512$ & $10.366 \pm 0.022$ & $0.445 \pm 0.036$ & +0.20 & & \\
\hline GJ 3077 & 12.899 & 1.441 & 3396 & 3326 & $3361 \pm 35$ & $\mathrm{dM} 3.5$ & $63.5922 \pm 0.1506$ & $11.916 \pm 0.025$ & $0.274 \pm 0.028$ & -0.12 & 1 & \\
\hline GJ 3131 & 11.194 & 1.275 & 3520 & 3498 & $3509 \pm 11$ & $\mathrm{dM} 2.7$ & $46.7620 \pm 0.0610$ & $9.543 \pm 0.023$ & $0.627 \pm 0.025$ & +0.18 & & Binary? \\
\hline GJ 3135 & 12.159 & 1.379 & 3445 & 3408 & $3427 \pm 19$ & $\mathrm{dM} 3.1$ & $106.7094 \pm 0.0566$ & $12.300 \pm 0.021$ & $0.203 \pm 0.013$ & -0.34 & & Subdwarf? \\
\hline GJ 3136 & 12.461 & 1.487 & 3356 & 3327 & $3342 \pm 14$ & $\mathrm{dM} 3.6$ & $58.5983 \pm 0.0783$ & $11.300 \pm 0.023$ & $0.377 \pm 0.022$ & -0.14 & 1 & \\
\hline GJ 3139 & 11.770 & 1.333 & 3476 & 3380 & $3427 \pm 47$ & $\mathrm{dM} 3.1$ & $50.4815 \pm 0.0517$ & $10.286 \pm 0.022$ & $0.514 \pm 0.063$ & +0.47 & 4 & Binary? \\
\hline GJ 3141 & 12.25 & 1.344 & 3469 & 3412 & $3441 \pm 29$ & dM3.1 & $67.4452 \pm 0.0439$ & $11.395 \pm 0.021$ & $0.301 \pm 0.025$ & -0.30 & & \\
\hline GJ $3148 \mathrm{~A}$ & 12.027 & 1.468 & 3372 & 3248 & $3310 \pm 62$ & $\mathrm{dM} 3.7$ & $74.5545 \pm 0.0585$ & $11.389 \pm 0.022$ & $0.385 \pm 0.062$ & +0.09 & & \\
\hline GJ $3160 \mathrm{~A}$ & 12.76 & 1.292 & 3506 & 3544 & $3525 \pm 19$ & $\mathrm{dM} 2.6$ & $39.0544 \pm 0.1819$ & $10.718 \pm 0.030$ & $0.358 \pm 0.019$ & -0.07 & 3 & \\
\hline GJ 3160B & 12.76 & 1.292 & 3506 & 3544 & $3525 \pm 19$ & $\mathrm{dM} 2.6$ & $39.0544 \pm 0.1819$ & $10.718 \pm 0.030$ & $0.358 \pm 0.019$ & -0.07 & 3 & \\
\hline
\end{tabular}


Table 2:: continued.

\begin{tabular}{|c|c|c|c|c|c|c|c|c|c|c|c|c|}
\hline Star & $\underset{(\mathrm{mag})}{\mathrm{V}}$ & $\begin{array}{c}(\mathrm{R}-\mathrm{I})_{C} \\
(\mathrm{mag})\end{array}$ & $\begin{array}{c}T_{e f f}{ }^{1} \\
(\mathrm{~K})\end{array}$ & $\begin{array}{c}T_{\text {eff }}^{2} \\
(\mathrm{~K})\end{array}$ & $T_{\text {eff }} \pm 3 \sigma^{3}$ & $\begin{array}{l}\text { Spect. } \\
\text { Type }\end{array}$ & $\begin{array}{c}\pi \\
(\mathrm{m} ")\end{array}$ & $\begin{array}{c}M_{V} \\
(\mathrm{mag})\end{array}$ & $\begin{array}{c}R_{\star} \\
\left(R_{\odot}\right)\end{array}$ & {$[\mathrm{M} / \mathrm{H}]$} & $\begin{array}{l}\text { Hi. Res. } \\
\text { Spectra }\end{array}$ & Comments \\
\hline GJ 3189 & 12.67 & 1.318 & 3487 & 3384 & $3435 \pm 51$ & sdM3.1 & $86.8162 \pm 0.1176$ & $12.363 \pm 0.023$ & $0.195 \pm 0.025$ & -0.60 & 3 & \\
\hline GJ 3190 & 13.056 & 1.475 & 3366 & - & $3366 \pm 26$ & $\mathrm{dM} 3.4$ & $43.1528 \pm 0.0732$ & $11.231 \pm 0.024$ & $0.373 \pm 0.030$ & -0.13 & & \\
\hline GJ 3195 & 12.55 & 1.363 & 3456 & 3524 & $3490 \pm 34$ & $\mathrm{dM} 2.8$ & $42.5088 \pm 0.0482$ & $10.692 \pm 0.022$ & $0.380 \pm 0.035$ & -0.17 & 1 & \\
\hline GJ 3205 & 11.874 & 1.257 & 3536 & 3483 & $3510 \pm 26$ & $\mathrm{dM} 2.7$ & $55.2336 \pm 0.0555$ & $10.585 \pm 0.022$ & $0.387 \pm 0.022$ & -0.10 & & \\
\hline GJ 3214 & 13.307 & 1.413 & 3420 & - & $3420 \pm 26$ & $\mathrm{dM} 3.2$ & $26.1362 \pm 0.2456$ & $10.393 \pm 0.040$ & $0.496 \pm 0.044$ & -0.06 & & \\
\hline GJ 3239A & 13.49 & 1.275 & 3520 & - & $3520 \pm 26$ & $\mathrm{dM} 2.6$ & $25.7892 \pm 0.0540$ & $10.550 \pm 0.025$ & $0.389 \pm 0.018$ & & 1 & \\
\hline GJ 3239B & 13.49 & 1.275 & 3520 & - & $3520 \pm 26$ & $\mathrm{dM} 2.6$ & $25.7892 \pm 0.0540$ & $10.550 \pm 0.025$ & $0.389 \pm 0.018$ & & 1 & \\
\hline GJ 3242 & 12.70 & 1.336 & 3474 & 3459 & $3467 \pm 8$ & $\mathrm{dM} 2.9$ & $38.8970 \pm 0.0760$ & $10.650 \pm 0.024$ & $0.404 \pm 0.017$ & +0.00 & 1 & \\
\hline GJ 3263A & 13.131 & 1.499 & 3346 & 3290 & $3318 \pm 28$ & $\mathrm{dM} 3.7$ & $64.1068 \pm 0.1189$ & $11.413 \pm 0.024$ & $0.375 \pm 0.033$ & +0.09 & & \\
\hline GJ 3263B & 13.131 & 1.499 & 3346 & 3290 & $3318 \pm 28$ & $\mathrm{dM} 3.7$ & $64.1068 \pm 0.1189$ & $11.413 \pm 0.024$ & $0.375 \pm 0.033$ & +0.09 & & \\
\hline GJ 3271 & 12.180 & 1.313 & 3490 & 3525 & $3508 \pm 17$ & $\mathrm{dM} 2.7$ & $51.3915 \pm 0.0421$ & $10.734 \pm 0.022$ & $0.362 \pm 0.017$ & & 1 & \\
\hline GJ 3279 & 11.8 & 1.324 & 3482 & 3547 & $3514 \pm 33$ & $\mathrm{dM} 2.6$ & $60.3928 \pm 0.0331$ & $10.705 \pm 0.021$ & $0.365 \pm 0.024$ & & 1 & \\
\hline GJ 3293 & 12.0 & 1.308 & 3493 & 3600 & $3546 \pm 53$ & $\mathrm{dM} 2.5$ & $49.4999 \pm 0.0525$ & $10.473 \pm 0.022$ & $0.391 \pm 0.036$ & +0.11 & 140 & \\
\hline GJ 3325 & 11.749 & 1.446 & 3391 & 3321 & $3356 \pm 35$ & $\mathrm{dM} 3.5$ & $108.3263 \pm 0.0500$ & $11.923 \pm 0.021$ & $0.276 \pm 0.027$ & -0.15 & & \\
\hline GJ 3332 & 11.19 & 1.450 & 3388 & 3496 & $3442 \pm 54$ & $\mathrm{dM} 3.0$ & $50.6488 \pm 0.1230$ & $9.713 \pm 0.025$ & $0.652 \pm$ & +0.20 & & Binary? \\
\hline GJ 3333A & 12.557 & 1.376 & 3445 & 3467 & $3456 \pm 11$ & $\mathrm{dM} 3.0$ & $86.0992 \pm 0.3080$ & $12.232 \pm 0.028$ & $0.199 \pm 0.008$ & & 1 & Subdwarf? \\
\hline GJ 3333B & 12.557 & 1.376 & 3445 & 3467 & $3456 \pm 11$ & $\mathrm{dM} 3.0$ & $86.0992 \pm 0.3080$ & $12.232 \pm 0.028$ & $0.199 \pm 0.008$ & & 1 & Subdwarf? \\
\hline GJ 3335 & 12.465 & 1.326 & 3481 & 3531 & $3506 \pm 25$ & $\mathrm{dM} 2.7$ & $33.6173 \pm 0.0730$ & $10.098 \pm 0.025$ & $0.487 \pm 0.028$ & & 2 & \\
\hline GJ 3341 & 12.115 & 1.268 & 3526 & 3525 & $3526 \pm 1$ & $\mathrm{dM} 2.6$ & $42.3099 \pm 0.0517$ & $10.247 \pm 0.023$ & $0.444 \pm 0.012$ & +0.09 & & \\
\hline GJ 3349 & 12.234 & 1.288 & 3509 & 3585 & $3547 \pm 38$ & $\mathrm{dM} 2.4$ & $46.6729 \pm 0.0660$ & $10.579 \pm 0.023$ & $0.372 \pm 0.027$ & -0.16 & 1 & \\
\hline GJ 3354B & 12.08 & 1.351 & 3464 & 3416 & $3440 \pm 24$ & $\mathrm{dM} 3.1$ & $45.1508 \pm 0.0806$ & $10.353 \pm 0.024$ & $0.487 \pm 0.037$ & -0.13 & & \\
\hline GJ 3388 & 12.829 & 1.464 & 3376 & 3372 & $3374 \pm 2$ & $\mathrm{dM} 3.4$ & $67.6281 \pm 0.0787$ & $11.980 \pm 0.023$ & $0.260 \pm 0.009$ & & & \\
\hline GJ $3404 \mathrm{~A}$ & 11.73 & 1.412 & 3421 & 3475 & $3448 \pm 27$ & dM3.0 & $66.3502 \pm 0.0702$ & $10.839 \pm 0.022$ & $0.384 \pm 0.031$ & +0.11 & 2 & \\
\hline GJ 3406A & 12.34 & 1.438 & 3398 & 3340 & $3369 \pm 29$ & $\mathrm{dM} 3.4$ & $52.8722 \pm 0.0436$ & $10.956 \pm 0.022$ & $0.421 \pm 0.036$ & -0.01 & 1 & \\
\hline GJ $3412 \mathrm{~A}$ & 11.781 & 1.393 & 3435 & 3417 & $3426 \pm 9$ & $\mathrm{dM} 3.1$ & $92.3604 \pm 0.5017$ & $11.608 \pm 0.032$ & $0.280 \pm 0.014$ & & 1 & \\
\hline GJ 3412B & 11.781 & 1.393 & 3435 & 3417 & $3426 \pm 9$ & $\mathrm{dM} 3.1$ & $92.3604 \pm 0.5017$ & $11.608 \pm 0.032$ & $0.280 \pm 0.014$ & & 1 & \\
\hline GJ 3439 & 13.369 & 1.462 & 3378 & 3407 & $3393 \pm 15$ & dM3.3 & $39.0280 \pm 0.0603$ & $11.326 \pm 0.023$ & $0.339 \pm 0.020$ & -0.77 & & \\
\hline GJ 3447B & 13.48 & 1.288 & 3509 & - & $3509 \pm 26$ & $\mathrm{dM} 2.7$ & $37.2447 \pm 0.2483$ & $11.335 \pm 0.034$ & $0.275 \pm 0.018$ & & & Subdwarf? \\
\hline GJ $3448 \mathrm{~B}$ & 13.48 & 1.288 & 3509 & - & $3509 \pm 26$ & $\mathrm{dM} 2.7$ & $34.0574 \pm 0.0810$ & $11.141 \pm 0.025$ & $0.300 \pm 0.018$ & -0.09 & & \\
\hline GJ 3452 & 11.308 & 1.250 & 3542 & 3507 & $3525 \pm 17$ & $\mathrm{dM} 2.6$ & $78.1854 \pm 0.0575$ & $10.774 \pm 0.022$ & $0.349 \pm 0.016$ & +0.06 & 1 & \\
\hline GJ 3459 & 11.712 & 1.408 & 3424 & 3381 & $3402 \pm 21$ & sdM3.3 & $95.8401 \pm 0.0413$ & $11.620 \pm 0.021$ & $0.291 \pm 0.020$ & -0.20 & 4 & \\
\hline GJ 3473 & 13.74 & 1.492 & 3352 & 3332 & $3342 \pm 10$ & $\mathrm{dM} 3.6$ & $36.5150 \pm 0.0498$ & $11.552 \pm 0.023$ & $0.336 \pm 0.017$ & -0.07 & & \\
\hline GJ 3492 & 11.85 & 1.329 & 3479 & 3425 & $3452 \pm 27$ & $\mathrm{dM} 3.0$ & $64.0731 \pm 0.0346$ & $10.883 \pm 0.021$ & $0.373 \pm 0.030$ & & 7 & \\
\hline GJ 3501B & 12.36 & 1.313 & 3490 & - & $3490 \pm 26$ & $\mathrm{dM} 2.8$ & $39.9747 \pm 0.1231$ & $10.369 \pm 0.027$ & $0.441 \pm 0.035$ & & & \\
\hline GJ 3502B & 12.36 & 1.313 & 3490 & - & $3490 \pm 26$ & $\mathrm{dM} 2.8$ & $39.6980 \pm 0.0584$ & $10.354 \pm 0.023$ & $0.444 \pm 0.034$ & -0.26 & & \\
\hline GJ 3506 & 11.82 & 1.377 & 3446 & 3460 & $3453 \pm 7$ & $\mathrm{dM} 3.0$ & $76.8538 \pm 0.0366$ & $11.248 \pm 0.021$ & $0.315 \pm 0.013$ & & 1 & \\
\hline GJ 3508 & 11.749 & 1.319 & 3486 & 3526 & $3506 \pm 20$ & $\mathrm{dM} 2.7$ & $56.1032 \pm 0.0594$ & $10.494 \pm 0.022$ & $0.406 \pm 0.020$ & -0.11 & 8 & \\
\hline GJ 3528 & 11.728 & 1.300 & 3499 & 3485 & $3492 \pm 7$ & $\mathrm{dM} 2.8$ & $49.0247 \pm 0.0545$ & $10.180 \pm 0.022$ & $0.479 \pm 0.019$ & -0.13 & 11 & \\
\hline GJ 3553 & 13.335 & 1.394 & 3435 & 3290 & $3363 \pm 73$ & $\mathrm{dM} 3.5$ & $56.6354 \pm 0.0610$ & $12.100 \pm 0.022$ & $0.251 \pm 0.045$ & -0.28 & & Subdwarf? \\
\hline GJ 3563 & 11.953 & 1.311 & 3491 & 3449 & $3470 \pm 21$ & $\mathrm{dM} 2.9$ & $58.6635 \pm 0.0609$ & $10.795 \pm 0.022$ & $0.376 \pm 0.025$ & & 1 & \\
\hline GJ 3564 & 12.060 & 1.451 & 3387 & 3365 & $3376 \pm 11$ & $\mathrm{dM} 3.4$ & $69.4962 \pm 0.0922$ & $11.270 \pm 0.023$ & $0.359 \pm 0.018$ & +0.16 & & \\
\hline GJ 3598 & 12.46 & 1.292 & 3506 & 3576 & $3541 \pm 35$ & $\mathrm{dM} 2.5$ & $51.6917 \pm 0.0505$ & $11.027 \pm 0.022$ & $0.305 \pm 0.021$ & & 7 & \\
\hline GJ 3604 & 13.00 & 1.450 & 3388 & - & $3388 \pm 26$ & $\mathrm{dM} 3.3$ & $44.5606 \pm 0.0483$ & $11.245 \pm 0.022$ & $0.355 \pm 0.028$ & +0.01 & & \\
\hline GJ 3607 & 12.712 & 1.400 & 3430 & 3342 & $3386 \pm 44$ & $\mathrm{dM} 3.3$ & $36.4557 \pm 0.0820$ & $10.521 \pm 0.025$ & $0.498 \pm 0.059$ & -0.57 & & Binary? \\
\hline GJ 3634 & 11.95 & 1.291 & 3507 & 3496 & $3501 \pm 6$ & $\mathrm{dM} 2.7$ & $49.0440 \pm 0.0756$ & $10.403 \pm 0.023$ & $0.426 \pm 0.014$ & -0.15 & & \\
\hline GJ 3643 & 12.369 & 1.346 & 3467 & 3411 & $3439 \pm 28$ & $\mathrm{dM} 3.1$ & $55.9143 \pm 0.0441$ & $11.107 \pm 0.022$ & $0.345 \pm 0.028$ & -0.11 & 52 & \\
\hline GJ 3659 & 13.08 & 1.433 & 3403 & - & $3403 \pm 26$ & $\mathrm{dM} 3.3$ & $55.6175 \pm 0.0815$ & $11.806 \pm 0.023$ & $0.267 \pm 0.021$ & +0.09 & & \\
\hline GJ 3700 & 12.345 & 1.338 & 3473 & 3555 & $3514 \pm 41$ & $\mathrm{dM} 2.6$ & $40.3009 \pm 0.1135$ & $10.372 \pm 0.026$ & $0.425 \pm 0.034$ & +0.20 & & \\
\hline GJ 3708A & 11.709 & 1.376 & 3447 & 3390 & $3418 \pm 28$ & $\mathrm{dM} 3.2$ & $78.3453 \pm 0.0578$ & $11.179 \pm 0.022$ & $0.347 \pm 0.029$ & -0.01 & 18 & \\
\hline GJ 3709B & 12.621 & 1.475 & 3366 & 3341 & $3354 \pm 13$ & $\mathrm{dM} 3.5$ & $78.5165 \pm 0.1094$ & $12.096 \pm 0.023$ & $0.256 \pm 0.014$ & -0.01 & & \\
\hline GJ $3728 \mathrm{~A}$ & 11.94 & 1.262 & 3532 & 3664 & $3598 \pm 66$ & $\mathrm{dM} 2.2$ & $51.4652 \pm 0.0590$ & $10.501 \pm 0.022$ & $0.363 \pm 0.035$ & -0.19 & & \\
\hline GJ 3728B & 11.94 & 1.262 & 3532 & 3664 & $3598 \pm 66$ & $\mathrm{dM} 2.2$ & $51.4652 \pm 0.0590$ & $10.501 \pm 0.022$ & $0.363 \pm 0.035$ & -0.19 & & \\
\hline GJ 3736 & 13.330 & 1.403 & 3428 & 3500 & $3464 \pm 36$ & $\mathrm{dM} 2.9$ & $35.4728 \pm 0.0585$ & $11.079 \pm 0.024$ & $0.333 \pm 0.033$ & +0.00 & & \\
\hline
\end{tabular}


Table 2:: continued.

\begin{tabular}{|c|c|c|c|c|c|c|c|c|c|c|c|c|}
\hline Star & $\begin{array}{c}\mathrm{V} \\
(\mathrm{mag})\end{array}$ & $\begin{array}{c}(\mathrm{R}-\mathrm{I})_{C} \\
(\mathrm{mag})\end{array}$ & $\begin{array}{c}T_{\text {eff }}{ }^{1} \\
(\mathrm{~K})\end{array}$ & $\begin{array}{c}T_{e f f}^{2} \\
(\mathrm{~K})\end{array}$ & $T_{(\mathrm{K})}^{T_{\text {eff }} \pm 3 \sigma^{3}}$ & $\begin{array}{l}\text { Spect. } \\
\text { Type }\end{array}$ & $\begin{array}{c}\pi \\
(\mathrm{m} ")\end{array}$ & $\begin{array}{c}M_{V} \\
\text { (mag) }\end{array}$ & $\begin{array}{c}R_{\star} \\
\left(R_{\odot}\right)\end{array}$ & {$[\mathrm{M} / \mathrm{H}]$} & $\begin{array}{l}\text { Hi. Res. } \\
\text { Spectra }\end{array}$ & Comments \\
\hline GJ 3747 & 12.600 & 1.401 & 3430 & 3520 & $3475 \pm 35$ & $\mathrm{dM} 2.9$ & $47.9305 \pm 0.0744$ & $11.003 \pm 0.023$ & $0.339 \pm 0.032$ & -0.27 & 1 & \\
\hline GJ 3748 & 11.773 & 1.313 & 3490 & 3477 & $3483 \pm 6$ & $\mathrm{dM} 2.8$ & $49.4421 \pm 0.0309$ & $10.243 \pm 0.021$ & $0.473 \pm 0.018$ & -0.22 & & \\
\hline GJ 3756 & 14.410 & 1.487 & 3356 & & $3356 \pm 26$ & dM3.5 & $46.3892 \pm 0.0446$ & $12.742 \pm 0.022$ & $0.189 \pm 0.015$ & -0.39 & & Subdwarf? \\
\hline GJ 3764 & 12.86 & 1.493 & 3351 & 3473 & $3412 \pm 61$ & $\mathrm{dM} 3.2$ & $59.9101 \pm 0.0475$ & $11.747 \pm 0.022$ & $0.270 \pm 0.041$ & -0.09 & & \\
\hline GJ 3787 & 13.1 & 1.428 & 3407 & - & $3407 \pm 26$ & $\mathrm{dM} 3.2$ & $56.6081 \pm 0.1004$ & $11.864 \pm 0.024$ & $0.258 \pm 0.021$ & -0.12 & & Subdwarf? \\
\hline GJ 3795 & 12.5 & 1.367 & 3453 & 3469 & $3461 \pm 8$ & $\mathrm{dM} 2.9$ & $43.7076 \pm 0.0451$ & $10.703 \pm 0.022$ & $0.399 \pm 0.017$ & +0.18 & & \\
\hline GJ 3803A & 13.59 & 1.250 & 3542 & 3481 & $3512 \pm 31$ & sdM2.7 & $41.1 \pm 6.3$ & $11.662 \pm 0.330$ & $0.235 \pm 0.050$ & -0.62 & & \\
\hline GJ 3803B & 13.59 & 1.250 & 3542 & 3481 & $3512 \pm 31$ & $\mathrm{sdM} 2.7$ & $41.1 \pm 6.3$ & $11.662 \pm 0.330$ & $0.235 \pm 0.050$ & -0.62 & & \\
\hline GJ 3804 & 11.866 & 1.498 & 3347 & 3283 & $3315 \pm 32$ & dM3.7 & $89.2264 \pm 0.0781$ & $11.618 \pm 0.022$ & $0.343 \pm 0.033$ & +0.09 & & \\
\hline GJ 3810 & 12.730 & 1.413 & 3420 & 3437 & $3428 \pm 9$ & dM3.1 & $47.4932 \pm 0.0596$ & $11.113 \pm 0.023$ & $0.351 \pm 0.016$ & +0.13 & & \\
\hline GJ 3817 & 12.264 & 1.475 & 3366 & 3289 & $3328 \pm 38$ & dM3.6 & $106.9246 \pm 0.0869$ & $12.409 \pm 0.022$ & $0.233 \pm 0.025$ & -0.13 & & Subdwarf? \\
\hline GJ 3836 & 11.82 & 1.347 & 3467 & 3449 & $3458 \pm 9$ & $\mathrm{dM} 3.0$ & $58.9719 \pm 0.0373$ & $10.673 \pm 0.021$ & $0.407 \pm 0.018$ & +0.18 & & \\
\hline GJ 3842 & 11.79 & 1.313 & 3490 & 3495 & $3493 \pm 2$ & $\mathrm{dM} 2.8 \mathrm{e}$ & $27.8268 \pm 0.0533$ & $9.012 \pm 0.024$ & $0.819 \pm 0.025$ & +0.03 & & PMS? \\
\hline GJ 3843 & 12.95 & 1.259 & 3534 & 3283 & $3409 \pm 125$ & $\mathrm{dM} 3.2$ & $74.5644 \pm 0.0808$ & $12.313 \pm 0.022$ & $0.209 \pm 0.063$ & -0.02 & & Subdwarf? \\
\hline GJ 3846 & 12.257 & 1.399 & 3431 & 3381 & $3406 \pm 25$ & sdM3.3 & $77.0884 \pm 0.0805$ & $11.692 \pm 0.022$ & $0.280 \pm 0.022$ & -0.13 & 2 & \\
\hline GJ 3884 & 12.738 & 1.484 & 3359 & 3339 & $3349 \pm 10$ & dM3.5 & $30.6541 \pm 0.4095$ & $10.170 \pm 0.049$ & $0.627 \pm 0.039$ & +0.37 & & Binary? \\
\hline GJ 3892 & 11.473 & 1.340 & 3472 & 3403 & $3437 \pm 34$ & $\mathrm{dM} 3.1$ & $68.4948 \pm 0.0644$ & $10.651 \pm 0.022$ & $0.427 \pm 0.040$ & -0.36 & 2 & \\
\hline GJ 3895 & 13.45 & 1.427 & 3408 & 3450 & $3429 \pm 21$ & $\mathrm{dM} 3.2$ & $41.2283 \pm 0.0697$ & $11.526 \pm 0.024$ & $0.290 \pm 0.020$ & -0.24 & & \\
\hline GJ 3913 & 12.722 & 1.487 & 3356 & 3286 & $3321 \pm 35$ & dM3.7 & $54.5662 \pm 0.0698$ & $11.407 \pm 0.023$ & $0.374 \pm 0.038$ & +0.07 & & \\
\hline GJ 3916A & 12.032 & 1.306 & 3495 & 3564 & $3529 \pm 34$ & $\mathrm{dM} 2.6$ & $62.5155 \pm 0.2773$ & $11.012 \pm 0.030$ & $0.311 \pm 0.022$ & +0.02 & 27 & \\
\hline GJ 3916B & 12.032 & 1.306 & 3495 & 3564 & $3529 \pm 34$ & $\mathrm{dM} 2.6$ & $62.5155 \pm 0.2773$ & $11.012 \pm 0.030$ & $0.311 \pm 0.022$ & +0.02 & 27 & \\
\hline GJ 3929 & 12.685 & 1.462 & 3378 & 3421 & $3400 \pm 21$ & sdM3.3 & $63.2240 \pm 0.0380$ & $11.689 \pm 0.021$ & $0.283 \pm 0.020$ & -0.65 & & \\
\hline GJ 3972 & 11.649 & 1.328 & 3480 & 3482 & $3481 \pm 1$ & $\mathrm{dM} 2.8$ & $63.4066 \pm 0.0355$ & $10.660 \pm 0.021$ & $0.392 \pm 0.011$ & +0.22 & 1 & \\
\hline GJ 3992 & 11.602 & 1.478 & 3364 & 3331 & $3347 \pm 16$ & dM3.5 & $83.1461 \pm 0.0458$ & $11.201 \pm 0.021$ & $0.391 \pm 0.024$ & & & \\
\hline GJ 4004 & 12.1 & 1.455 & 3384 & 3462 & $3423 \pm 39$ & sdM2.9 & $78.4702 \pm 0.0418$ & $11.574 \pm 0.201$ & $0.286 \pm 0.057$ & & 2 & \\
\hline GJ 4005 & 12.167 & 1.326 & 3481 & 3630 & $3555 \pm 74$ & $\mathrm{dM} 2.4$ & $48.4723 \pm 0.0721$ & $10.594 \pm 0.023$ & $0.366 \pm 0.044$ & +0.01 & & \\
\hline GJ 4011 & 12.816 & 1.363 & 3456 & - & $3456 \pm 26$ & dM3.0 & $35.9774 \pm 0.1122$ & $10.596 \pm 0.027$ & $0.423 \pm 0.034$ & +0.14 & & \\
\hline GJ 4019B & 12.735 & 1.425 & 3409 & 3455 & $3432 \pm 23$ & $\mathrm{dM} 3.1$ & $36.5025 \pm 0.1808$ & $10.547 \pm 0.031$ & $0.452 \pm 0.035$ & +0.15 & & \\
\hline GJ 4022 & 12.55 & 1.407 & 3425 & 3425 & $3425 \pm 1$ & dM3.1 & $39.2080 \pm 0.1043$ & $10.517 \pm 0.026$ & $0.464 \pm 0.014$ & +0.30 & & \\
\hline GJ 4026 & 12.67 & 1.408 & 3424 & 3427 & $3425 \pm 2$ & dM3.1 & $66.7587 \pm 0.0467$ & $11.793 \pm 0.022$ & $0.258 \pm 0.008$ & -0.49 & & Subdwarf? \\
\hline GJ 4040 & 11.770 & 1.437 & 3399 & 3421 & $3410 \pm 11$ & $\mathrm{dM} 3.2 \mathrm{e}$ & $71.4754 \pm 0.0354$ & $11.041 \pm 0.021$ & $0.375 \pm 0.018$ & +0.04 & 1 & \\
\hline GJ $4048 \mathrm{~A}$ & 11.87 & 1.363 & 3456 & 3390 & $3423 \pm 33$ & $\mathrm{dM} 3.1$ & $88.0532 \pm 0.0348$ & $11.594 \pm 0.021$ & $0.284 \pm 0.026$ & -0.13 & 1 & \\
\hline GJ 4070 & 11.358 & 1.391 & 3436 & 3424 & $3430 \pm 6$ & $\mathrm{dM} 3.1$ & $92.5881 \pm 0.0332$ & $11.191 \pm 0.021$ & $0.337 \pm 0.013$ & & & \\
\hline GJ 4117 & 12.20 & 1.291 & 3507 & 3540 & $3523 \pm 17$ & $\mathrm{dM} 2.6$ & $65.7479 \pm 0.0324$ & $11.289 \pm 0.021$ & $0.276 \pm 0.013$ & +0.78 & & \\
\hline GJ 4124 & 12.410 & 1.351 & 3464 & 3445 & $3455 \pm 10$ & dM3.0 & $58.5 \pm 3.4$ & $11.246 \pm 0.143$ & $0.314 \pm 0.033$ & -0.20 & 2 & \\
\hline GJ 4129 & 11.95 & 1.304 & 3496 & 3465 & $3480 \pm 15$ & $\mathrm{dM} 2.8$ & $64.2331 \pm 0.0542$ & $10.989 \pm 0.022$ & $0.338 \pm 0.019$ & -0.26 & 5 & \\
\hline GJ 4149 & 11.96 & 1.288 & 3509 & 3585 & $3547 \pm 38$ & $\mathrm{dM} 2.5$ & $47.6010 \pm 0.0601$ & $10.348 \pm 0.023$ & $0.414 \pm 0.030$ & +0.04 & 5 & \\
\hline GJ 4154 & 11.5 & 1.344 & 3469 & 3431 & $3450 \pm 19$ & dM3.0 & $93.7195 \pm 0.0348$ & $11.359 \pm 0.201$ & $0.301 \pm 0.047$ & -0.18 & & \\
\hline GJ 4161B & 12.24 & 1.462 & 3378 & 3790 & $3584 \pm 206$ & $\mathrm{dM} 2.2$ & $40.7975 \pm 0.0904$ & $10.293 \pm 0.025$ & $0.406 \pm 0.127$ & & 1 & \\
\hline GJ 4176A & 12.29 & 1.376 & 3447 & - & $3447 \pm 26$ & dM3.0 & $45.9752 \pm 0.5444$ & $10.603 \pm 0.046$ & $0.429 \pm 0.038$ & +0.02 & 1 & \\
\hline GJ 4187 & 12.11 & 1.300 & 3499 & - & $3499 \pm 26$ & $\mathrm{dM} 2.7$ & $33.4496 \pm 0.0448$ & $9.732 \pm 0.023$ & $0.582 \pm 0.045$ & & 2 & Binary? \\
\hline GJ 4197 & 12.21 & 1.313 & 3490 & 3593 & $3541 \pm 52$ & $\mathrm{dM} 2.5$ & $47.4904 \pm 0.1027$ & $10.593 \pm 0.025$ & $0.372 \pm 0.034$ & +0.03 & & \\
\hline GJ 4205 & 11.775 & 1.351 & 3464 & 3493 & $3479 \pm 15$ & $\mathrm{dM} 2.8$ & $56.7717 \pm 0.0425$ & $10.546 \pm 0.022$ & $0.415 \pm 0.023$ & & 1 & \\
\hline GJ 4223 & 13.33 & 1.499 & 3346 & 3289 & $3318 \pm 29$ & $\mathrm{dM} 3.7$ & $48.5192 \pm 0.0388$ & $11.760 \pm 0.022$ & $0.320 \pm 0.029$ & -0.16 & & \\
\hline GJ $4231 \mathrm{~A}$ & 12.126 & 1.388 & 3439 & 3517 & $3478 \pm 39$ & $\mathrm{dM} 2.8 \mathrm{e}$ & $32.79 \pm 5.65$ & $9.705 \pm 0.365$ & $0.612 \pm 0.179$ & -0.06 & 7 & PMS? \\
\hline GJ $4231 \mathrm{~B}$ & 12.126 & 1.388 & 3439 & 3517 & $3478 \pm 39$ & $\mathrm{dM} 2.8 \mathrm{e}$ & $32.79 \pm 5.65$ & $9.705 \pm 0.365$ & $0.612 \pm 0.179$ & -0.06 & 7 & PMS? \\
\hline GJ 4248 & 11.80 & 1.485 & 3357 & 3333 & $3345 \pm 12$ & $\mathrm{dM} 3.6$ & $134.29 \pm 1.31$ & $12.440 \pm 0.041$ & $0.222 \pm 0.014$ & -0.07 & & Subdwarf? \\
\hline GJ 4251 & 13.41 & 1.462 & 3378 & - & $3378 \pm 26$ & $\mathrm{dM} 3.4$ & $48.6356 \pm 0.0356$ & $11.845 \pm 0.022$ & $0.275 \pm 0.022$ & -0.39 & & \\
\hline GJ $4282 \mathrm{~A}$ & 12.41 & 1.401 & 3430 & 3400 & $3415 \pm 15$ & $\mathrm{dM} 3.2 \mathrm{e}$ & $38.2385 \pm 0.1244$ & $11.076 \pm 0.027$ & $0.366 \pm 0.017$ & -0.16 & 1 & \\
\hline
\end{tabular}


Table 2:: continued.

\begin{tabular}{|c|c|c|c|c|c|c|c|c|c|c|c|c|}
\hline Star & $\underset{(\mathrm{mag})}{\mathrm{V}}$ & $\begin{array}{c}(\mathrm{R}-\mathrm{I})_{C} \\
(\mathrm{mag})\end{array}$ & $\begin{array}{c}T_{\text {eff }}{ }^{1} \\
(\mathrm{~K})\end{array}$ & $\begin{array}{c}T_{\text {eff }}^{2} \\
(\mathrm{~K})\end{array}$ & $T_{\text {eff }} \pm 3 \sigma^{3}$ & $\begin{array}{l}\text { Spect. } \\
\text { Type }\end{array}$ & $\begin{array}{c}\pi \\
(\mathrm{m} ")\end{array}$ & $\begin{array}{c}M_{V} \\
(\mathrm{mag})\end{array}$ & $\begin{array}{c}R_{\star} \\
\left(R_{\odot}\right)\end{array}$ & {$[\mathrm{M} / \mathrm{H}]$} & $\begin{array}{l}\text { Hi. Res. } \\
\text { Spectra }\end{array}$ & Comments \\
\hline GJ 4282B & 12.41 & 1.401 & 3430 & 3400 & $3415 \pm 15$ & $\mathrm{dM} 3.2 \mathrm{e}$ & $39.9030 \pm 0.2214$ & $11.168 \pm 0.032$ & $0.350 \pm 0.017$ & -0.16 & 1 & \\
\hline GJ $4289 B$ & 12.64 & 1.475 & 3366 & - & $3366 \pm 26$ & $\mathrm{dM} 3.4$ & $84.4509 \pm 0.0736$ & $12.273 \pm 0.022$ & $0.231 \pm 0.019$ & -0.12 & & Subdwarf? \\
\hline GJ 4297 & 12.917 & 1.402 & 3429 & - & $3429 \pm 26$ & $\mathrm{dM} 3.1$ & $53.9253 \pm 0.0517$ & $11.576 \pm 0.022$ & $0.283 \pm 0.022$ & +0.16 & 1 & \\
\hline GJ 4312 & 12.45 & 1.401 & 3430 & 3432 & $3431 \pm 1$ & $\mathrm{dM} 3.1$ & $64.9753 \pm 0.0383$ & $11.514 \pm 0.021$ & $0.290 \pm 0.008$ & & 1 & \\
\hline GJ $4327 \mathrm{~A}$ & 12.668 & 1.326 & 3481 & 3484 & $3483 \pm 2$ & $\mathrm{dM} 2.8$ & $59.6911 \pm 0.2426$ & $11.548 \pm 0.029$ & $0.260 \pm 0.005$ & +0.26 & 1 & \\
\hline GJ 4327B & 12.668 & 1.326 & 3481 & 3484 & $3483 \pm 2$ & $\mathrm{dM} 2.8$ & $59.6911 \pm 0.2426$ & $11.548 \pm 0.029$ & $0.260 \pm 0.005$ & +0.26 & 1 & \\
\hline GJ 4336 & 12.657 & 1.364 & 3462 & 3629 & $3546 \pm 84$ & $\mathrm{dM} 2.5$ & $40.6466 \pm 0.0548$ & $10.702 \pm 0.023$ & $0.352 \pm 0.047$ & & 1 & \\
\hline GJ 4337A & 12.130 & 1.450 & 3388 & 3404 & $3396 \pm 8$ & $\mathrm{dM} 3.3$ & $67.0425 \pm 0.0504$ & $11.262 \pm 0.022$ & $0.348 \pm 0.015$ & & 1 & \\
\hline GJ 4362 & 12.34 & 1.356 & 3461 & 3443 & $3452 \pm 9$ & dM3.0 & $38.2541 \pm 0.0647$ & $10.253 \pm 0.024$ & $0.499 \pm 0.022$ & & 4 & Binary? \\
\hline GJ 4368 & 11.50 & 1.362 & 3457 & 3374 & $3415 \pm 41$ & $\mathrm{dM} 3.2$ & $49.0870 \pm 0.1429$ & $9.955 \pm 0.026$ & $0.613 \pm 0.069$ & +0.16 & 1 & Binary? \\
\hline GJ 4379B & 12.99 & 1.413 & 3420 & 3313 & $3366 \pm 53$ & dM3.4 & $50.8558 \pm 0.2842$ & $11.522 \pm 0.032$ & $0.326 \pm 0.046$ & & 2 & \\
\hline GJ 9119B & 12.87 & 1.438 & 3398 & - & $3398 \pm 26$ & $\mathrm{dM} 3.3$ & $25.4650 \pm 0.1190$ & $9.900 \pm 0.030$ & $0.648 \pm 0.054$ & +0.18 & & Binary? \\
\hline GJ 9120 & 13.095 & 1.452 & 3386 & 3459 & $3423 \pm 36$ & $\mathrm{dM} 3.1$ & $38.7647 \pm 0.0488$ & $11.037 \pm 0.023$ & $0.367 \pm 0.036$ & +0.18 & & \\
\hline GJ 9141 & 13.633 & 1.487 & 3356 & - & $3356 \pm 26$ & $\mathrm{dM} 3.5$ & $39.5821 \pm 0.0595$ & $11.620 \pm 0.023$ & $0.317 \pm 0.026$ & -0.26 & & \\
\hline GJ 9238 & 13.795 & 1.257 & 3536 & 3549 & $3543 \pm 6$ & $\mathrm{sdM} 2.5$ & $25.0677 \pm 0.0604$ & $10.791 \pm 0.025$ & $0.339 \pm 0.011$ & -1.11 & & \\
\hline GJ 9453B & 13.47 & 1.425 & 3409 & - & $3409 \pm 26$ & $\mathrm{dM} 3.2$ & $25.5021 \pm 0.0302$ & $10.503 \pm 0.023$ & $0.481 \pm 0.038$ & +0.35 & & \\
\hline GJ 9652AA & 12.306 & 1.456 & 3383 & 3317 & $3350 \pm 33$ & $\mathrm{dM} 3.5$ & $55.2372 \pm 0.0643$ & $11.017 \pm 0.023$ & $0.423 \pm 0.034$ & -0.16 & 1 & \\
\hline GJ $9652 \mathrm{AB}$ & 12.306 & 1.456 & 3383 & 3317 & $3350 \pm 33$ & $\mathrm{dM} 3.5$ & $55.2372 \pm 0.0643$ & $11.017 \pm 0.023$ & $0.423 \pm 0.034$ & -0.16 & 1 & \\
\hline GJ 9677B & 13.535 & 1.425 & 3409 & - & $3409 \pm 26$ & $\mathrm{dM} 3.2$ & $36.7282 \pm 0.0297$ & $11.360 \pm 0.022$ & $0.324 \pm 0.026$ & -0.37 & & \\
\hline GJ 9801B & 13.52 & 1.407 & 3425 & 3360 & $3392 \pm 32$ & dM3.3 & $37.1269 \pm 0.1646$ & $11.368 \pm 0.030$ & $0.333 \pm 0.032$ & -0.18 & & \\
\hline Gl 12 & 12.61 & 1.415 & 3418 & 3354 & $3386 \pm 32$ & $\mathrm{sdM} 3.3$ & $81.8711 \pm 0.0866$ & $12.176 \pm 0.022$ & $0.232 \pm 0.021$ & -0.26 & 3 & Subdwarf? \\
\hline Gl $22 \mathrm{~A}$ & 10.327 & 1.262 & 3532 & 3531 & $3532 \pm 1$ & $\mathrm{dM} 2.5$ & $101.4335 \pm 0.4733$ & $10.358 \pm 0.030$ & $0.419 \pm 0.013$ & -0.33 & 1 & \\
\hline Gl 22B & 12.19 & - & - & 3339 & $3339 \pm 26$ & $\mathrm{dM} 3.6$ & $100.4054 \pm 0.0701$ & $12.199 \pm 0.022$ & $0.251 \pm 0.021$ & -0.33 & 1 & Subdwarf? \\
\hline GL 26 & 11.155 & 1.272 & 3523 & 3629 & $3576 \pm 53$ & $\mathrm{dM} 2.3$ & $78.9551 \pm 0.0355$ & $10.642 \pm 0.021$ & $0.349 \pm 0.032$ & & 1 & \\
\hline Gl 46 & 11.786 & 1.473 & 3368 & 3343 & $3356 \pm 13$ & $\mathrm{dM} 3.5$ & $83.5181 \pm 0.4599$ & $11.395 \pm 0.032$ & $0.352 \pm 0.021$ & +0.15 & & \\
\hline Gl 47 & 10.865 & 1.250 & 3542 & 3472 & $3507 \pm 35$ & $\mathrm{dM} 2.7$ & $95.0899 \pm 0.0372$ & $10.756 \pm 0.021$ & $0.359 \pm 0.025$ & +0.00 & & \\
\hline GL 48 & 9.995 & 1.363 & 3456 & 3379 & $3417 \pm 38$ & $\mathrm{dM} 3.2 \mathrm{e}$ & $121.4237 \pm 0.0339$ & $10.417 \pm 0.021$ & $0.493 \pm 0.051$ & +0.04 & 79 & \\
\hline Gl 53.1B & 13.60 & 1.450 & 3388 & 3257 & $3323 \pm 65$ & $\mathrm{dM} 3.7$ & $48.8481 \pm 0.1143$ & $12.044 \pm 0.025$ & $0.278 \pm 0.047$ & +0.16 & & \\
\hline Gl 54 & 9.824 & 1.383 & 3442 & 3428 & $3435 \pm 7$ & $\mathrm{dM} 3.1$ & $121.4487 \pm 1.1930$ & $10.246 \pm 0.041$ & $0.516 \pm 0.026$ & & 1 & Binary? \\
\hline Gl 70 & 10.915 & 1.287 & 3510 & 3463 & $3486 \pm 23$ & $\mathrm{dM} 2.8$ & $88.3392 \pm 0.0453$ & $10.646 \pm 0.021$ & $0.391 \pm 0.027$ & -0.16 & 4 & \\
\hline Gl $84 \mathrm{~A}$ & 10.942 & 1.290 & 3507 & 3496 & $3501 \pm 6$ & $\mathrm{dM} 2.7$ & $105.9466 \pm 0.1111$ & $11.067 \pm 0.022$ & $0.314 \pm 0.007$ & -0.08 & 16 & \\
\hline Gl $84 \mathrm{~B}$ & 10.942 & 1.290 & 3507 & 3496 & $3501 \pm 6$ & $\mathrm{dM} 2.7$ & $105.9466 \pm 0.1111$ & $11.067 \pm 0.022$ & $0.314 \pm 0.007$ & -0.08 & 16 & \\
\hline Gl $84.1 \mathrm{~B}$ & 12.792 & 1.466 & 3374 & 3400 & $3387 \pm 13$ & $\mathrm{dM} 3.3$ & $38.1477 \pm 0.1017$ & $10.699 \pm 0.026$ & $0.458 \pm 0.025$ & -0.18 & & \\
\hline Gl 94 & 12.556 & 1.475 & 3366 & 3357 & $3362 \pm 5$ & $\mathrm{dM} 3.5$ & $57.2495 \pm 0.0871$ & $11.345 \pm 0.023$ & $0.356 \pm 0.014$ & & 1 & \\
\hline Gl $100 \mathrm{C}$ & 12.84 & 1.351 & 3464 & - & $3464 \pm 26$ & $\mathrm{dM} 2.9$ & $50.8621 \pm 0.0542$ & $11.372 \pm 0.022$ & $0.291 \pm 0.023$ & -0.28 & & \\
\hline Gl 109 & 10.563 & 1.386 & 3440 & 3370 & $3405 \pm 35$ & $\mathrm{dM} 3.2$ & $130.1870 \pm 0.0605$ & $11.136 \pm 0.021$ & $0.362 \pm 0.035$ & -0.11 & 36 & \\
\hline Gl 118 & 11.382 & 1.332 & 3477 & 3412 & $3444 \pm 32$ & $\mathrm{dM} 3.0$ & $85.1443 \pm 0.0325$ & $11.033 \pm 0.021$ & $0.353 \pm 0.032$ & & 19 & \\
\hline GL 118.1B & 12.97 & 1.357 & 3460 & 3340 & $3400 \pm 60$ & $\mathrm{dM} 3.3$ & $34.7329 \pm 0.0322$ & $10.674 \pm 0.022$ & $0.452 \pm 0.068$ & -0.07 & 2 & \\
\hline Gl 119B & 11.65 & 1.313 & 3490 & 3600 & $3545 \pm 55$ & $\mathrm{dM} 2.5$ & $49.2873 \pm 0.0430$ & $10.114 \pm 0.022$ & $0.452 \pm 0.068$ & & & \\
\hline GL $120 \mathrm{~A}$ & 13.792 & 1.407 & 3425 & 3304 & $3365 \pm 61$ & $\mathrm{dM} 3.4$ & $60.9925 \pm 0.0908$ & $12.718 \pm 0.023$ & $0.188 \pm 0.026$ & -0.42 & & Subdwarf? \\
\hline GL $120 \mathrm{~B}$ & 13.792 & 1.407 & 3425 & 3304 & $3365 \pm 61$ & $\mathrm{dM} 3.4$ & $60.9925 \pm 0.0908$ & $12.718 \pm 0.023$ & $0.188 \pm 0.026$ & -0.42 & & Subdwarf? \\
\hline GL 121.1 & 11.792 & 1.304 & 3496 & 3473 & $3484 \pm 11$ & $\mathrm{dM} 2.8 \mathrm{e}$ & $52.4383 \pm 0.0787$ & $10.390 \pm 0.023$ & $0.442 \pm 0.021$ & -0.02 & 1 & \\
\hline Gl $140 \mathrm{C}$ & 11.89 & 1.288 & 3509 & 3505 & $3507 \pm 2$ & $\mathrm{dM} 2.7$ & $48.4516 \pm 0.0677$ & $10.317 \pm 0.023$ & $0.440 \pm 0.012$ & & 1 & \\
\hline GJ 143.3 & 12.263 & 1.266 & 3528 & 3490 & $3509 \pm 19$ & $\mathrm{dM} 2.7$ & $49.4528 \pm 0.1000$ & $10.734 \pm 0.024$ & $0.362 \pm 0.018$ & -0.05 & & \\
\hline Gl 145 & 11.47 & 1.339 & 3472 & 3447 & $3459 \pm 12$ & sdM3.0 & $91.1916 \pm 0.0338$ & $11.270 \pm 0.021$ & $0.308 \pm 0.015$ & -0.28 & 5 & \\
\hline Gl 163 & 11.811 & 1.416 & 3417 & 3333 & $3375 \pm 42$ & $\mathrm{dM} 3.4$ & $66.0714 \pm 0.0314$ & $10.911 \pm 0.021$ & $0.425 \pm 0.048$ & +0.03 & 119 & \\
\hline GL 173.1B & 14.19 & 1.401 & 3430 & 3380 & $3405 \pm 25$ & $\mathrm{dM} 3.3$ & $34.0366 \pm 0.1115$ & $11.850 \pm 0.027$ & $0.261 \pm 0.021$ & -0.33 & 1 & \\
\hline Gl 190 & 10.302 & 1.488 & 3356 & 3303 & $3329 \pm 26$ & $\mathrm{dM} 3.6$ & $108.7231 \pm 0.0942$ & $10.484 \pm 0.022$ & $0.564 \pm 0.044$ & +0.06 & & Binary? \\
\hline Gl 203 & 12.443 & 1.495 & 3349 & 3260 & $3305 \pm 45$ & $\mathrm{dM} 3.8$ & $97.9790 \pm 0.0952$ & $12.399 \pm 0.022$ & $0.245 \pm 0.031$ & -0.23 & & Subdwarf? \\
\hline Gl 204.2 & 12.010 & 1.343 & 3470 & 3400 & $3435 \pm 35$ & dM3.1 & $57.7585 \pm 0.0767$ & $10.818 \pm 0.023$ & $0.397 \pm 0.039$ & & 1 & \\
\hline Gl 207.1 & 11.53 & 1.335 & 3475 & 3448 & $3461 \pm 13$ & $\mathrm{dM} 2.9 \mathrm{e}$ & $63.5990 \pm 0.0889$ & $10.547 \pm 0.023$ & $0.428 \pm 0.022$ & +0.09 & 2 & \\
\hline Gl 226 & 10.50 & 1.309 & 3493 & 3531 & $3512 \pm 19$ & $\mathrm{dM} 2.6$ & $105.6941 \pm 0.0304$ & $10.620 \pm 0.021$ & $0.380 \pm 0.018$ & & 31 & \\
\hline Gl 226.1 & 13.694 & 1.381 & 3443 & - & $3443 \pm 26$ & $\mathrm{dM} 3.0$ & $44.7343 \pm 0.0788$ & $11.947 \pm 0.024$ & $0.232 \pm 0.018$ & -0.12 & & Subdwarf? \\
\hline
\end{tabular}


Table 2:: continued.

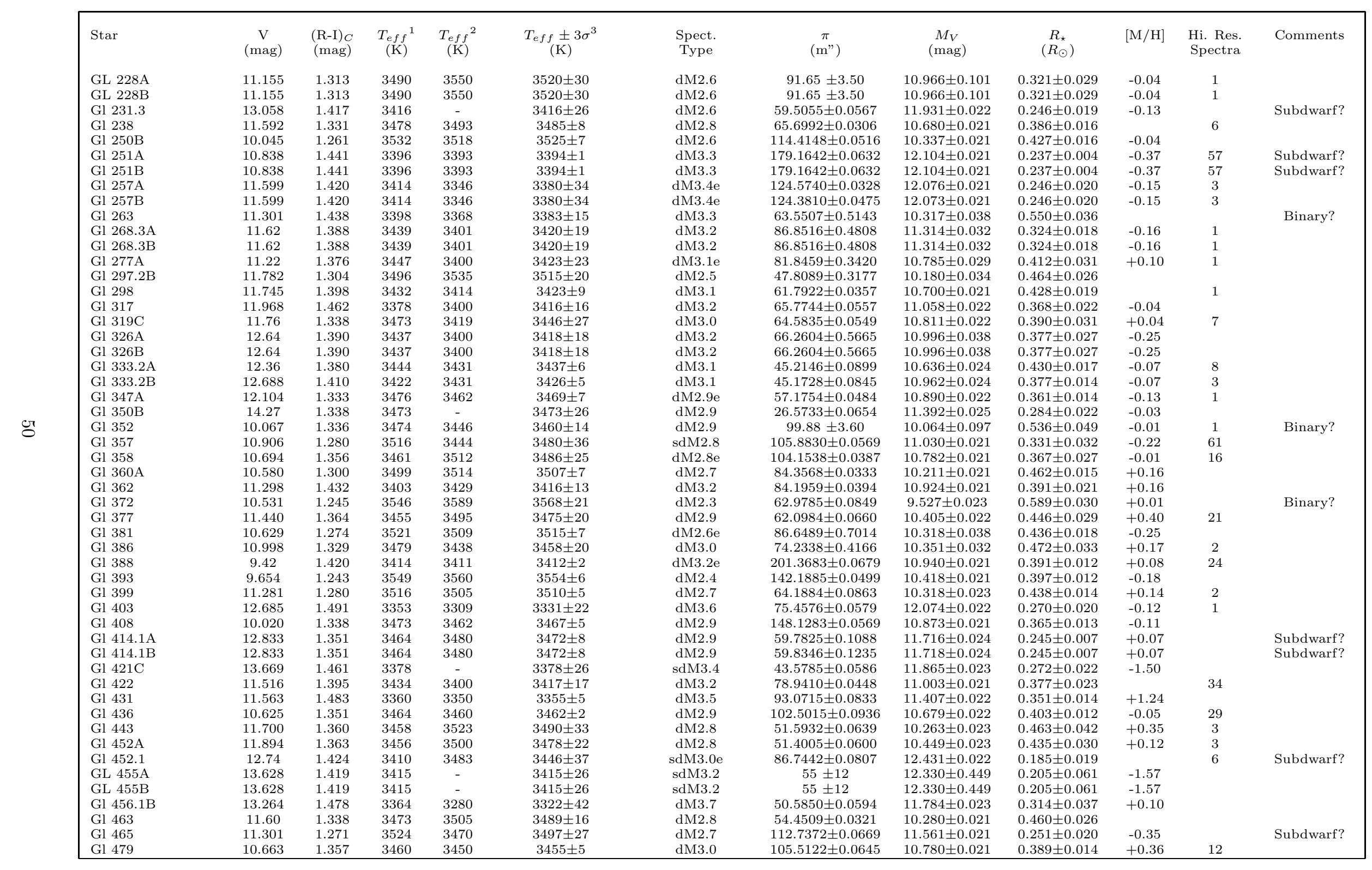


Table 2:: continued.

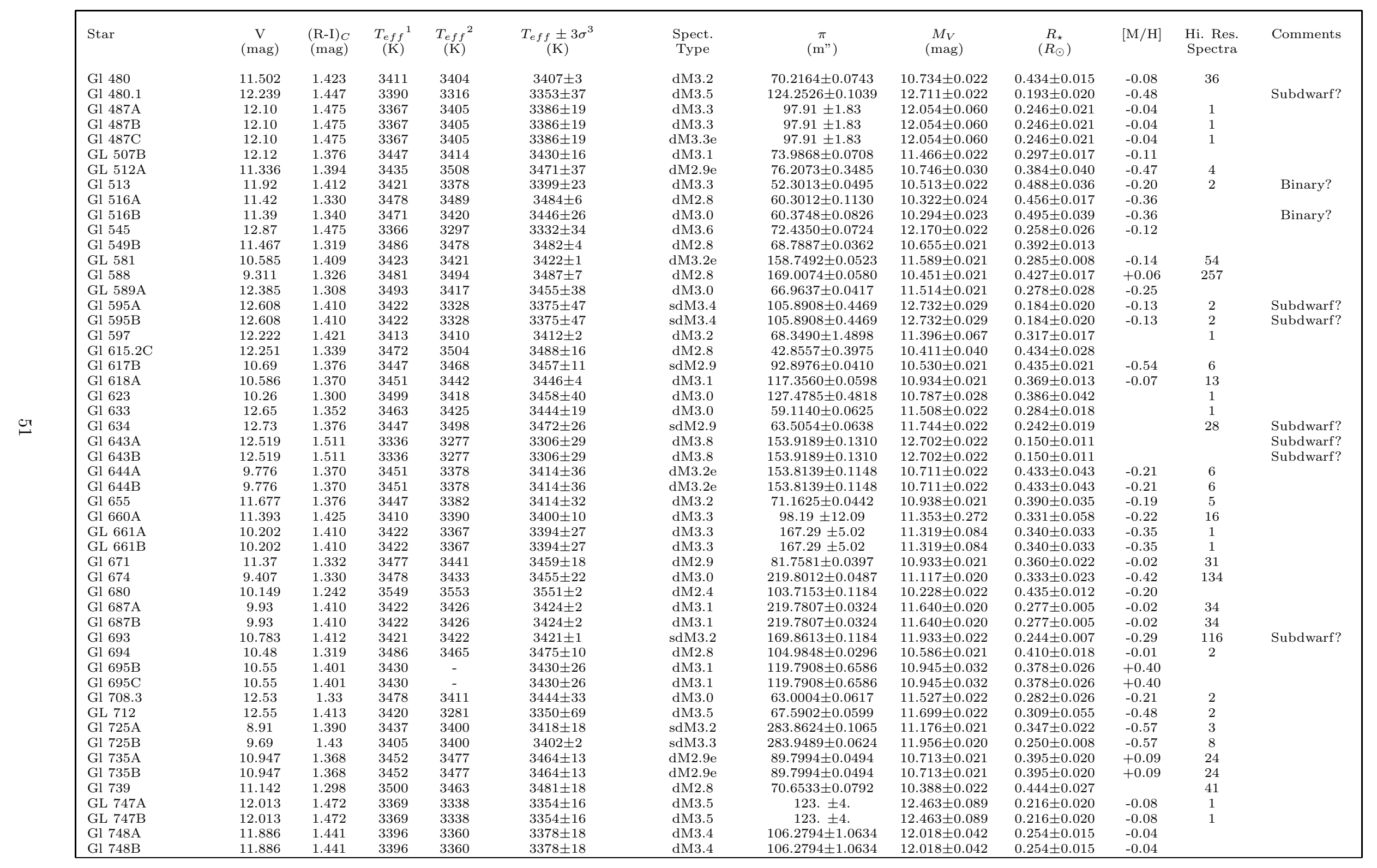


Table 2:: continued.

\begin{tabular}{|c|c|c|c|c|c|c|c|c|c|c|c|c|}
\hline Star & $\underset{(\mathrm{mag})}{\mathrm{V}}$ & $\begin{array}{c}(\mathrm{R}-\mathrm{I})_{C} \\
(\mathrm{mag})\end{array}$ & $\begin{array}{c}T_{e f f}{ }^{1} \\
(\mathrm{~K})\end{array}$ & $\begin{array}{c}T_{e f f}^{2} \\
(\mathrm{~K})\end{array}$ & $\begin{array}{c}T_{e f f} \pm 3 \sigma^{3} \\
(\mathrm{~K})\end{array}$ & $\begin{array}{l}\text { Spect. } \\
\text { Type }\end{array}$ & $\begin{array}{c}\pi \\
(\mathrm{m} ")\end{array}$ & $\begin{array}{c}M_{V} \\
(\mathrm{mag})\end{array}$ & $\begin{array}{c}R_{\star} \\
\left(R_{\odot}\right)\end{array}$ & {$[\mathrm{M} / \mathrm{H}]$} & $\begin{array}{l}\text { Hi. Res. } \\
\text { Spectra }\end{array}$ & Comments \\
\hline Gl $752 \mathrm{~A}$ & 9.115 & 1.326 & 3481 & 3494 & $3487 \pm 7$ & $\mathrm{dM} 2.8$ & $169.1590 \pm 0.0520$ & $10.256 \pm 0.021$ & $0.467 \pm 0.018$ & +0.05 & 109 & \\
\hline Gl $754.1 \mathrm{~B}$ & 11.658 & 1.270 & 3525 & 3361 & $3443 \pm 18$ & dM3.0 & $95.2517 \pm 0.0748$ & $11.552 \pm 0.022$ & $0.279 \pm 0.017$ & -0.22 & & \\
\hline Gl $774 \mathrm{~A}$ & 11.34 & 1.276 & 3520 & 3670 & $3595 \pm 75$ & $\mathrm{dM} 2.2$ & $77.7604 \pm 0.0536$ & $10.794 \pm 0.021$ & $0.318 \pm 0.038$ & & 1 & \\
\hline Gl $774 \mathrm{~B}$ & 12.28 & 1.413 & 3420 & - & $3420 \pm 26$ & $\mathrm{dM} 3.2$ & $77.8623 \pm 0.0670$ & $11.737 \pm 0.022$ & $0.267 \pm 0.021$ & & 1 & \\
\hline GL 781.1A & 12.22 & 1.395 & 3434 & 3412 & $3423 \pm 11$ & $\mathrm{dM} 3.1 \mathrm{e}$ & $67.9318 \pm 0.0837$ & $11.380 \pm 0.023$ & $0.313 \pm 0.015$ & -0.07 & 2 & \\
\hline Gl 781.1B & 12.25 & 1.351 & 3464 & 3445 & $3455 \pm 10$ & dM3.0 & $68.0088 \pm 0.0852$ & $11.413 \pm 0.023$ & $0.291 \pm 0.013$ & -0.07 & & \\
\hline GL 791 & 11.433 & 1.357 & 3460 & 3611 & $3535 \pm 76$ & $\mathrm{dM} 2.5 \mathrm{e}$ & $69.8796 \pm 0.0685$ & $10.655 \pm 0.022$ & $0.364 \pm 0.045$ & +0.06 & 4 & \\
\hline Gl 793 & 10.55 & 1.388 & 3439 & 3365 & $3402 \pm 37$ & dM3.3 & $123.7545 \pm 0.0219$ & $11.013 \pm 0.020$ & $0.385 \pm 0.039$ & -0.09 & 2 & \\
\hline GL 811.1 & 11.487 & 1.331 & 3478 & 3491 & $3484 \pm 7$ & $\mathrm{dM} 2.8 \mathrm{e}$ & $62.1155 \pm 0.0721$ & $10.453 \pm 0.023$ & $0.429 \pm 0.017$ & +0.11 & 5 & \\
\hline Gl $812 \mathrm{~A}$ & 11.893 & 1.450 & 3388 & 3399 & $3394 \pm 6$ & dM3.3 & $62.2541 \pm 0.0958$ & $10.864 \pm 0.023$ & $0.419 \pm 0.017$ & +0.12 & & \\
\hline GL 813 & 12.013 & 1.288 & 3509 & 3456 & $3482 \pm 26$ & $\mathrm{dM} 2.8$ & $69.3112 \pm 0.0676$ & $11.217 \pm 0.022$ & $0.303 \pm 0.023$ & -0.32 & 4 & \\
\hline GL 816 & 11.219 & 1.319 & 3486 & 3462 & $3474 \pm 12$ & $\mathrm{dM} 2.9$ & $67.1402 \pm 0.1217$ & $10.354 \pm 0.024$ & $0.457 \pm 0.023$ & +0.00 & 20 & \\
\hline Gl $829 \mathrm{~A}$ & 10.304 & 1.470 & 3371 & 3358 & $3364 \pm 6$ & dM3.5 & $147.6126 \pm 0.0979$ & $11.903 \pm 0.021$ & $0.274 \pm 0.007$ & -0.08 & 1 & \\
\hline Gl 829B & 10.304 & 1.470 & 3371 & 3358 & $3364 \pm 6$ & $\mathrm{dM} 3.5$ & $147.6126 \pm 0.0979$ & $11.903 \pm 0.021$ & $0.274 \pm 0.007$ & -0.08 & 1 & \\
\hline GL 836.4 & 12.83 & 1.395 & 3434 & 3631 & $3532 \pm 99$ & $\mathrm{dM} 2.5$ & $31.0623 \pm 0.0589$ & $10.291 \pm 0.024$ & $0.432 \pm 0.067$ & -0.06 & & \\
\hline GL 843 & 12.03 & 1.394 & 3435 & 3306 & $3370 \pm 64$ & dM3.4 & $77 \pm 10$ & $11.462 \pm 0.285$ & $0.332 \pm 0.103$ & -0.16 & 5 & \\
\hline Gl 844 & 10.653 & 1.288 & 3509 & 3553 & $3531 \pm 22$ & $\mathrm{dM} 2.5$ & $61.79 \pm 2.23$ & $9.608 \pm 0.097$ & $0.593 \pm 0.053$ & +1.30 & 8 & \\
\hline Gl 849 & 10.366 & 1.427 & 3408 & 3357 & $3382 \pm 25$ & dM3.3 & $113.6000 \pm 0.0463$ & $10.643 \pm 0.021$ & $0.474 \pm 0.037$ & +0.25 & 13 & \\
\hline GL 856A & 11.838 & 1.401 & 3430 & 3440 & $3435 \pm 5$ & $\mathrm{dM} 3.1 \mathrm{e}$ & $65.4804 \pm 0.1172$ & $10.919 \pm 0.024$ & $0.379 \pm 0.009$ & +0.04 & 2 & \\
\hline GL 856B & 11.838 & 1.401 & 3430 & 3440 & $3435 \pm 5$ & $\mathrm{dM} 3.1 \mathrm{e}$ & $65.8608 \pm 0.1018$ & $10.931 \pm 0.023$ & $0.377 \pm 0.009$ & +0.04 & 2 & \\
\hline GL $860 A$ & 9.645 & 1.487 & 3362 & 3433 & $3398 \pm 35$ & $\mathrm{dM} 3.3 \mathrm{e}$ & $249.3926 \pm 0.1653$ & $11.629 \pm 0.021$ & $0.292 \pm 0.029$ & -0.13 & 1 & \\
\hline GL $860 \mathrm{~B}$ & 10.30 & 1.49 & 3354 & 3263 & $3308 \pm 45$ & sdM3.7e & $249.9668 \pm 0.7414$ & $12.289 \pm 0.026$ & $0.255 \pm 0.032$ & -0.13 & & \\
\hline GL 875.1 & 11.645 & 1.401 & 3430 & 3490 & $3460 \pm 30$ & $\mathrm{dM} 2.9 \mathrm{e}$ & $66.4170 \pm 0.0672$ & $10.756 \pm 0.022$ & $0.390 \pm 0.033$ & +0.11 & 1 & \\
\hline Gl 877 & 10.377 & 1.346 & 3467 & 3488 & $3477 \pm 11$ & $\mathrm{dM} 2.8$ & $116.4127 \pm 0.0291$ & $10.707 \pm 0.021$ & $0.387 \pm 0.018$ & +0.00 & 8 & \\
\hline Gl 878 & 12.78 & 1.438 & 3398 & 3436 & $3417 \pm 19$ & $\mathrm{dM} 3.2$ & $56.1633 \pm 0.0390$ & $11.527 \pm 0.022$ & $0.296 \pm 0.019$ & +0.12 & 1 & \\
\hline Gl 889B & 13.662 & 1.475 & 3366 & 3320 & $3343 \pm 23$ & dM3.6 & $45.3005 \pm 0.0532$ & $11.943 \pm 0.023$ & $0.280 \pm 0.021$ & -0.04 & & \\
\hline Gl $897 \mathrm{~A}$ & 11.148 & 1.315 & 3489 & - & $3489 \pm 26$ & $\mathrm{dM} 2.8 \mathrm{e}$ & $77.5 \pm 9$ & $10.595 \pm 0.259$ & $0.398 \pm 0.074$ & & & \\
\hline Gl 897B & 11.148 & 1.315 & 3489 & - & $3489 \pm 26$ & $\mathrm{dM} 2.8 \mathrm{e}$ & $77.5 \pm 9$ & $10.595 \pm 0.259$ & $0.398 \pm 0.074$ & & & \\
\hline GL 898B & 11.18 & 1.326 & 3481 & - & $3481 \pm 26$ & $\mathrm{dM} 2.8$ & $68.6979 \pm 0.0581$ & $10.368 \pm 0.022$ & $0.449 \pm 0.029$ & & 50 & \\
\hline GL $898 \mathrm{C}$ & 11.18 & 1.326 & 3481 & - & $3481 \pm 26$ & $\mathrm{dM} 2.8$ & $68.6979 \pm 0.0581$ & $10.368 \pm 0.022$ & $0.449 \pm 0.029$ & & 50 & \\
\hline Gl 899 & 11.163 & 1.256 & 3537 & 3463 & $3500 \pm 37$ & $\mathrm{dM} 2.7$ & $71.3741 \pm 0.0496$ & $10.431 \pm 0.022$ & $0.421 \pm 0.041$ & -0.01 & & \\
\hline GL $905.2 \mathrm{~A}$ & 11.70 & 1.388 & 3439 & - & $3439 \pm 26$ & $\mathrm{dM} 3.1$ & $53.6996 \pm 0.0917$ & $10.350 \pm 0.024$ & $0.489 \pm 0.039$ & +0.06 & 1 & \\
\hline Gl 912 & 11.154 & 1.259 & 3534 & 3580 & $3557 \pm 23$ & $\mathrm{dM} 2.4$ & $56.1438 \pm 0.0964$ & $9.901 \pm 0.024$ & $0.502 \pm 0.027$ & +0.12 & & Binary? \\
\hline L $977-17$ & 13.45 & 1.401 & 3430 & - & $3430 \pm 26$ & dM3.1 & $44.3301 \pm 0.0728$ & $11.683 \pm 0.024$ & $0.269 \pm 0.021$ & -0.76 & & \\
\hline L $1197-67$ & 13.67 & 1.288 & 3509 & - & $3509 \pm 26$ & $\mathrm{dM} 2.7$ & $29.0860 \pm 0.0398$ & $10.988 \pm 0.023$ & $0.322 \pm 0.019$ & -0.49 & & \\
\hline L $1197-68$ & 13.55 & 1.288 & 3509 & - & $3509 \pm 26$ & $\mathrm{dM} 2.7$ & $29.0084 \pm 0.0398$ & $10.863 \pm 0.023$ & $0.341 \pm 0.020$ & -0.49 & & \\
\hline LP $24-330$ & 13.93 & 1.376 & 3447 & - & $3447 \pm 26$ & dM3.0 & $25.0142 \pm 0.0343$ & $10.921 \pm 0.023$ & $0.370 \pm 0.029$ & +0.28 & & \\
\hline LP $222-50$ & 13.569 & 1.340 & 3471 & 3435 & $3453 \pm 18$ & dM3.0 & $33.1625 \pm 0.0278$ & $11.172 \pm 0.022$ & $0.326 \pm 0.020$ & & & \\
\hline LP $640-77$ & 15.275 & 1.376 & 3447 & - & $3447 \pm 26$ & dM3.0 & $10.5563 \pm 0.0868$ & $10.393 \pm 0.038$ & $0.472 \pm 0.040$ & -0.37 & & \\
\hline LP $873-20$ & 13.951 & 1.438 & 3398 & - & $3398 \pm 26$ & $\mathrm{sdM} 3.3$ & $38.3098 \pm 0.0934$ & $11.868 \pm 0.025$ & $0.262 \pm 0.021$ & -0.62 & & \\
\hline LDS 2332B & 13.755 & 1.363 & 3456 & - & $3456 \pm 26$ & dM3.0 & $25.3188 \pm 0.0338$ & $10.772 \pm 0.023$ & $0.390 \pm 0.030$ & -0.08 & & \\
\hline LDS 3733B & 15.03 & 1.388 & 3439 & - & $3439 \pm 26$ & $\mathrm{dM} 3.1$ & $17.4658 \pm 0.0565$ & $11.241 \pm 0.027$ & $0.324 \pm 0.026$ & +0.22 & & \\
\hline LDS $6225 \mathrm{~B}$ & 15.50 & 1.351 & 3464 & - & $3464 \pm 26$ & $\mathrm{sdM} 2.9$ & $25.8185 \pm 0.0854$ & $12.560 \pm 0.027$ & $0.169 \pm 0.013$ & -0.56 & & \\
\hline LHS 137 & 13.360 & 1.275 & 3520 & - & $3520 \pm 26$ & $\mathrm{dM} 2.6$ & $42.5086 \pm 0.0490$ & $11.502 \pm 0.023$ & $0.251 \pm 0.015$ & -0.38 & & Subdwarf? \\
\hline LHS 156 & 14.901 & 1.244 & 3547 & 3550 & $3549 \pm 2$ & sdM2.4 & $31.2485 \pm 0.0432$ & $12.375 \pm 0.023$ & $0.162 \pm 0.005$ & -1.00 & & \\
\hline LHS 218 & 14.835 & 1.331 & 3478 & - & $3478 \pm 26$ & sdM2.8 & $24.9829 \pm 0.0425$ & $11.823 \pm 0.024$ & $0.231 \pm 0.018$ & +0.34 & & Subdwarf? \\
\hline LHS 272 & 13.456 & 1.364 & 3455 & 3401 & $3428 \pm 27$ & $\mathrm{sdM} 3.1$ & $74.5686 \pm 0.0440$ & $12.819 \pm 0.021$ & $0.160 \pm 0.013$ & -0.67 & 1 & \\
\hline LHS 297 & 13.239 & 1.319 & 3486 & - & $3486 \pm 26$ & $\mathrm{dM} 2.8$ & $26.8106 \pm 0.3480$ & $10.381 \pm 0.048$ & $0.442 \pm 0.040$ & -0.05 & & \\
\hline LHS 320 & 14.015 & 1.250 & 3542 & 3600 & $3571 \pm 29$ & $\mathrm{dM} 2.3$ & $25.8405 \pm 0.0639$ & $11.077 \pm 0.025$ & $0.288 \pm 0.018$ & -0.60 & & \\
\hline LHS 375 & 15.68 & 1.425 & 3410 & 3500 & $3455 \pm 45$ & sdM3.0 & $31.8503 \pm 0.0726$ & $13.196 \pm 0.025$ & $0.128 \pm 0.015$ & -1.10 & 2 & \\
\hline LHS 1037 & 13.002 & 1.450 & 3388 & - & $3388 \pm 26$ & dM3.3 & $47.7575 \pm 0.0677$ & $11.397 \pm 0.023$ & $0.331 \pm 0.027$ & & 1 & \\
\hline LHS 1048 & 13.34 & 1.294 & 3504 & - & $3504 \pm 26$ & $\mathrm{dM} 2.7$ & $25.2464 \pm 0.0415$ & $10.351 \pm 0.024$ & $0.435 \pm 0.025$ & & & \\
\hline LHS 1062 & 12.876 & 1.450 & 3388 & 3380 & $3384 \pm 4$ & $\mathrm{dM} 3.3$ & $40.4233 \pm 0.0602$ & $10.909 \pm 0.023$ & $0.418 \pm 0.015$ & -0.37 & 1 & \\
\hline
\end{tabular}


Table 2:: continued.

\begin{tabular}{|c|c|c|c|c|c|c|c|c|c|c|c|c|}
\hline Star & $\underset{(\mathrm{mag})}{\mathrm{V}}$ & $\begin{array}{c}(\mathrm{R}-\mathrm{I})_{C} \\
(\mathrm{mag})\end{array}$ & $\begin{array}{c}T_{e f f}^{1} \\
(\mathrm{~K})\end{array}$ & $\begin{array}{c}T_{e f f}^{2} \\
(\mathrm{~K})\end{array}$ & $T_{\text {eff }} \pm 3 \sigma^{3}$ & $\begin{array}{l}\text { Spect. } \\
\text { Type }\end{array}$ & $\begin{array}{c}\pi \\
(\mathrm{m} ")\end{array}$ & $\begin{array}{c}M_{V} \\
(\mathrm{mag})\end{array}$ & $\begin{array}{c}R_{\star} \\
\left(R_{\odot}\right)\end{array}$ & {$[\mathrm{M} / \mathrm{H}]$} & $\begin{array}{l}\text { Hi. Res. } \\
\text { Spectra }\end{array}$ & Comments \\
\hline LHS 1104 & 12.56 & 1.288 & 3509 & 3512 & $3511 \pm 2$ & $\mathrm{dM} 2.7$ & $41.2833 \pm 0.0784$ & $10.639 \pm 0.024$ & $0.377 \pm 0.011$ & +0.02 & & \\
\hline LHS 1162 & 15.415 & 1.475 & 3366 & - & $3366 \pm 26$ & $\mathrm{dM} 3.4$ & $25.9524 \pm 0.0439$ & $12.486 \pm 0.024$ & $0.209 \pm 0.017$ & +0.16 & & Subdwarf? \\
\hline LHS 1169 & 11.961 & 1.401 & 3430 & 3392 & $3411 \pm 19$ & $\mathrm{dM} 3.2$ & $76.0648 \pm 0.0462$ & $11.367 \pm 0.021$ & $0.322 \pm 0.021$ & & 1 & \\
\hline LHS 1185 & 13.50 & 1.402 & 3429 & - & $3429 \pm 26$ & $\mathrm{dM} 3.1$ & $30.2113 \pm 0.0805$ & $10.901 \pm 0.026$ & $0.386 \pm 0.031$ & +0.29 & & \\
\hline LHS 1202 & 12.858 & 1.288 & 3509 & - & $3509 \pm 26$ & $\mathrm{dM} 2.7$ & $51.9024 \pm 0.0289$ & $11.434 \pm 0.021$ & $0.262 \pm 0.015$ & -0.27 & & Subdwarf? \\
\hline LHS 1293 & 13.640 & 1.356 & 3461 & 3300 & $3381 \pm 81$ & dM3.4 & $31.2774 \pm 0.0329$ & $11.116 \pm 0.022$ & $0.382 \pm 0.076$ & +0.20 & & \\
\hline LHS 1306 & 13.43 & 1.275 & 3520 & - & $3520 \pm 26$ & $\mathrm{dM} 2.6$ & $26.2977 \pm 0.5126$ & $10.530 \pm 0.062$ & $0.393 \pm 0.030$ & +0.04 & & \\
\hline LHS 1333 & 13.670 & 1.399 & 3431 & - & $3431 \pm 26$ & dM3.1 & $18.8902 \pm 0.7948$ & $10.051 \pm 0.109$ & $0.569 \pm 0.070$ & -0.46 & & Binary? \\
\hline LHS 1383 & 13.386 & 1.313 & 3490 & - & $3490 \pm 26$ & $\mathrm{dM} 2.8$ & $20.9608 \pm 0.0658$ & $9.993 \pm 0.027$ & $0.524 \pm 0.041$ & +0.09 & & Binary? \\
\hline LHS 1384 & 14.35 & 1.285 & 3512 & - & $3512 \pm 26$ & $\mathrm{dM} 2.7$ & $34.9766 \pm 0.0272$ & $12.069 \pm 0.022$ & $0.195 \pm 0.011$ & -0.09 & & Subdwarf? \\
\hline LHS 1468 & 14.820 & 1.404 & 3427 & - & $3427 \pm 26$ & $\mathrm{dM} 3.1$ & $19.5310 \pm 0.0793$ & $11.274 \pm 0.029$ & $0.326 \pm 0.027$ & +0.13 & & \\
\hline LHS 1473 & 13.971 & 1.475 & 3366 & - & $3366 \pm 26$ & dM3.4 & $29.9217 \pm 0.0500$ & $11.351 \pm 0.024$ & $0.353 \pm 0.029$ & -0.10 & & \\
\hline LHS 1478 & 13.39 & 1.401 & 3430 & - & $3430 \pm 26$ & $\mathrm{dM} 3.1$ & $54.8329 \pm 0.0328$ & $12.085 \pm 0.021$ & $0.223 \pm 0.017$ & +0.13 & & Subdwarf? \\
\hline LHS 1495 & 12.93 & 1.413 & 3420 & - & $3420 \pm 26$ & $\mathrm{dM} 3.2$ & $38.3693 \pm 0.0445$ & $10.850 \pm 0.023$ & $0.402 \pm 0.032$ & +0.33 & 1 & \\
\hline LHS 1588 & 14.020 & 1.326 & 3481 & - & $3481 \pm 26$ & $\mathrm{dM} 2.8$ & $25.9094 \pm 0.0669$ & $11.087 \pm 0.026$ & $0.322 \pm 0.025$ & $\begin{array}{l}+0.59 \\
\end{array}$ & & \\
\hline LHS 1600 & 14.45 & 1.487 & 3356 & - & $3356 \pm 26$ & dM3.5 & $27.7204 \pm 0.0638$ & $11.664 \pm 0.025$ & $0.311 \pm 0.026$ & +0.07 & & \\
\hline LHS 1631 & 13.34 & 1.486 & 3357 & - & $3357 \pm 26$ & dM3.5 & $62.8041 \pm 0.0442$ & $12.330 \pm 0.022$ & $0.228 \pm 0.018$ & +0.36 & & Subdwarf? \\
\hline LHS 1728 & 13.628 & 1.499 & 3346 & - & $3346 \pm 26$ & dM3.5 & $44.2946 \pm 0.0540$ & $11.860 \pm 0.023$ & $0.290 \pm 0.024$ & $\begin{array}{l}7.00 \\
-2.02\end{array}$ & & \\
\hline LHS 1741 & 14.19 & 1.462 & 3378 & - & $3378 \pm 26$ & dM3.4 & $39.5589 \pm 0.0624$ & $12.176 \pm 0.023$ & $0.236 \pm 0.019$ & +0.57 & & Subdwarf? \\
\hline LHS 1778 & 13.264 & 1.376 & 3447 & - & $3447 \pm 26$ & dM3.0 & $30.8526 \pm 0.0551$ & $10.710 \pm 0.024$ & $0.408 \pm 0.032$ & -0.10 & & \\
\hline LHS 1820 & 14.92 & 1.363 & 3456 & - & $3456 \pm 26$ & $\mathrm{dM} 3.0$ & $17.1457 \pm 0.0529$ & $11.091 \pm 0.027$ & $0.337 \pm 0.027$ & -0.68 & & \\
\hline LHS 1847 & 13.2 & 1.270 & 3525 & - & $3525 \pm 26$ & $\mathrm{dM} 2.6$ & $30.6873 \pm 0.0300$ & $10.635 \pm 0.202$ & $0.372 \pm 0.055$ & +0.08 & & \\
\hline LHS 1873 & 13.137 & 1.441 & 3396 & - & $3396 \pm 26$ & dM3.3 & $40.9550 \pm 0.0445$ & $11.199 \pm 0.022$ & $0.358 \pm 0.029$ & -0.15 & & \\
\hline LHS 1983 & 14.77 & 1.438 & 3398 & - & $3398 \pm 26$ & dM3.3 & $21.0028 \pm 0.0555$ & $11.381 \pm 0.026$ & $0.328 \pm 0.027$ & -0.22 & & \\
\hline LHS 2184 & 14.520 & 1.420 & 3414 & - & $3414 \pm 26$ & $\mathrm{dM} 3.2$ & $31.2357 \pm 0.0592$ & $11.993 \pm 0.024$ & $0.240 \pm 0.019$ & -0.41 & & Subdwarf? \\
\hline LHS 2198 & 14.27 & 1.335 & 3475 & - & $3475 \pm 26$ & $\mathrm{dM} 2.9$ & $19.0497 \pm 0.2348$ & $10.669 \pm 0.047$ & $0.395 \pm 0.035$ & -0.70 & & \\
\hline LHS 2232 & 11.73 & 1.462 & 3378 & 3304 & $3341 \pm 37$ & dM3.1 & $99.4076 \pm 0.0978$ & $11.717 \pm 0.022$ & $0.312 \pm 0.033$ & +0.14 & & \\
\hline LHS 2292 & 12.214 & 1.313 & 3490 & 3493 & $3492 \pm 2$ & $\mathrm{dM} 2.8$ & $47.7233 \pm 0.0543$ & $10.608 \pm 0.022$ & $0.394 \pm 0.012$ & -0.10 & & \\
\hline LHS 2304 & 13.151 & 1.334 & 3476 & - & $3476 \pm 26$ & $\mathrm{dM} 2.9$ & $30.3566 \pm 0.0563$ & $10.562 \pm 0.024$ & $0.414 \pm 0.032$ & -0.20 & & \\
\hline LHS 2307 & 13.855 & 1.338 & 3473 & - & $3473 \pm 26$ & $\mathrm{dM} 2.9$ & $28.4912 \pm 0.1851$ & $11.129 \pm 0.034$ & $0.321 \pm 0.027$ & +0.34 & & \\
\hline LHS 2448 & 13.656 & 1.410 & 3422 & - & $3422 \pm 26$ & $\mathrm{dM} 3.2$ & $36.6821 \pm 0.0671$ & $11.478 \pm 0.024$ & $0.300 \pm 0.024$ & & & \\
\hline LHS 2458 & 14.175 & 1.326 & 3481 & - & $3481 \pm 26$ & $\mathrm{dM} 2.8$ & $21.8484 \pm 0.0362$ & $10.872 \pm 0.024$ & $0.356 \pm 0.028$ & & & \\
\hline LHS 2513 & 13.49 & 1.475 & 3366 & - & $3366 \pm 26$ & $\mathrm{dM} 3.4$ & $46.2366 \pm 0.0320$ & $11.815 \pm 0.022$ & $0.285 \pm 0.023$ & & & \\
\hline LHS 2659 & 13.225 & 1.363 & 3456 & - & $3456 \pm 26$ & dM3.0 & $42.8049 \pm 0.0353$ & $11.382 \pm 0.022$ & $0.294 \pm 0.023$ & & & \\
\hline LHS 2660 & 14.575 & 1.391 & 3437 & - & $3437 \pm 26$ & $\mathrm{dM} 3.1$ & $20.1149 \pm 0.0455$ & $11.093 \pm 0.025$ & $0.348 \pm 0.028$ & -0.26 & & \\
\hline LHS 2702 & 13.371 & 1.338 & 3473 & 3515 & $3494 \pm 21$ & $\mathrm{dM} 2.8$ & $25.8781 \pm 0.0270$ & $10.436 \pm 0.022$ & $0.425 \pm 0.028$ & -0.37 & & \\
\hline LHS 2748 & 14.041 & 1.499 & 3346 & - & $3346 \pm 26$ & $\mathrm{dM} 3.5$ & $35.1036 \pm 0.2175$ & $11.768 \pm 0.033$ & $0.302 \pm 0.027$ & & & \\
\hline LHS 2774 & 14.14 & 1.425 & 3409 & - & $3409 \pm 26$ & $\mathrm{sdM} 3.2$ & $32.6769 \pm 0.0475$ & $11.711 \pm 0.023$ & $0.276 \pm 0.022$ & -1.21 & & \\
\hline LHS 2942 & 14.27 & 1.401 & 3430 & - & $3430 \pm 26$ & $\mathrm{dM} 3.1$ & $30.8307 \pm 0.0842$ & $11.715 \pm 0.026$ & $0.265 \pm 0.021$ & & & Subdwarf? \\
\hline LHS 2949 & 13.369 & 1.364 & 3455 & - & $3455 \pm 26$ & dM3.0 & $30.5404 \pm 0.0559$ & $10.793 \pm 0.024$ & $0.387 \pm 0.030$ & & & \\
\hline LHS 3123 & 13.168 & 1.370 & 3451 & 3462 & $3457 \pm 6$ & dM3.0 & $34.9874 \pm 0.0673$ & $10.888 \pm 0.024$ & $0.369 \pm 0.014$ & -1.12 & & \\
\hline LHS 3267 & 13.825 & 1.434 & 3402 & - & $3402 \pm 26$ & dM3.3 & $35.2281 \pm 0.3351$ & $11.559 \pm 0.041$ & $0.300 \pm 0.027$ & & & \\
\hline LHS 3293 & 14.707 & 1.342 & 3470 & - & $3470 \pm 26$ & $\mathrm{dM} 2.9$ & $27.0996 \pm 0.0231$ & $11.872 \pm 0.022$ & $0.229 \pm 0.018$ & & & Subdwarf? \\
\hline LHS 3344 & 12.895 & 1.438 & 3399 & 3455 & $3427 \pm 28$ & $\mathrm{dM} 3.1$ & $31.2262 \pm 0.9523$ & $10.368 \pm 0.085$ & $0.495 \pm 0.057$ & & & \\
\hline LHS 3350 & 13.690 & 1.487 & 3356 & - & $3356 \pm 26$ & dM3.5 & $33.2660 \pm 0.0256$ & $11.300 \pm 0.022$ & $0.368 \pm 0.030$ & & 1 & \\
\hline LHS 3375 & 13.74 & 1.412 & 3421 & 3410 & $3416 \pm 6$ & $\mathrm{sdM} 3.2$ & $32.6960 \pm 0.0820$ & $11.312 \pm 0.025$ & $0.327 \pm 0.013$ & -0.56 & & \\
\hline LHS 3429 & 13.863 & 1.499 & 3346 & 3319 & $3333 \pm 14$ & $\mathrm{dM} 3.6$ & $48.6580 \pm 0.0695$ & $12.299 \pm 0.023$ & $0.242 \pm 0.014$ & -0.06 & & Subdwarf? \\
\hline LHS 3591 & 12.842 & 1.363 & 3456 & - & $3456 \pm 26$ & dM3.0 & $29.3106 \pm 0.1806$ & $10.177 \pm 0.033$ & $0.513 \pm 0.043$ & & & Binary? \\
\hline LHS 3616 & 13.7 & 1.338 & 3473 & - & $3473 \pm 26$ & $\mathrm{dM} 2.9$ & $38.4231 \pm 0.0701$ & $11.623 \pm 0.204$ & $0.255 \pm 0.044$ & & & Subdwarf? \\
\hline
\end{tabular}


Table 2:: continued.

\begin{tabular}{|c|c|c|c|c|c|c|c|c|c|c|c|c|}
\hline Star & $\begin{array}{c}\mathrm{V} \\
(\mathrm{mag})\end{array}$ & $\begin{array}{c}(\mathrm{R}-\mathrm{I})_{C} \\
(\mathrm{mag})\end{array}$ & $\begin{array}{c}T_{\text {eff }}{ }^{1} \\
(\mathrm{~K})\end{array}$ & $\begin{array}{c}T_{e f f}{ }^{2} \\
(\mathrm{~K})\end{array}$ & $T_{\text {eff }} \pm 3 \sigma^{3}$ & $\begin{array}{l}\text { Spect. } \\
\text { Type }\end{array}$ & $\begin{array}{c}\pi \\
(\mathrm{m} ")\end{array}$ & $\begin{array}{c}M_{V} \\
(\mathrm{mag})\end{array}$ & $\begin{array}{c}R_{\star} \\
\left(R_{\odot}\right)\end{array}$ & {$[\mathrm{M} / \mathrm{H}]$} & $\begin{array}{l}\text { Hi. Res. } \\
\text { Spectra }\end{array}$ & Comments \\
\hline LHS 3673 & 13.60 & 1.358 & 3459 & - & $3459 \pm 26$ & $\mathrm{dM} 3.0$ & $30.3772 \pm 0.0390$ & $11.013 \pm 0.023$ & $0.347 \pm 0.027$ & & & \\
\hline LHS 3699 & 12.94 & 1.288 & 3509 & - & $3509 \pm 26$ & $\mathrm{dM} 2.7$ & $34.1197 \pm 0.1427$ & $10.605 \pm 0.029$ & $0.384 \pm 0.024$ & & 1 & \\
\hline LHS 3747 & 15.31 & 1.468 & 3372 & - & $3372 \pm 26$ & $\mathrm{dM} 2.9$ & $23.2216 \pm 0.0766$ & $12.139 \pm 0.027$ & $0.243 \pm 0.020$ & & & Subdwarf? \\
\hline LHS 3784 & 14.25 & 1.422 & 3412 & - & $3412 \pm 26$ & $\mathrm{dM} 3.2$ & $27.1154 \pm 0.0611$ & $11.416 \pm 0.025$ & $0.314 \pm 0.025$ & & & \\
\hline LHS 3795 & 13.896 & 1.487 & 3356 & - & $3356 \pm 26$ & $\mathrm{dM} 3.5$ & $24.8627 \pm 0.2928$ & $10.874 \pm 0.045$ & $0.447 \pm 0.041$ & & & \\
\hline LHS 3835 & 14.075 & 1.363 & 3456 & - & $3456 \pm 26$ & $\mathrm{dM} 3.0$ & $20.5957 \pm 0.3053$ & $10.644 \pm 0.052$ & $0.414 \pm 0.038$ & & & \\
\hline LHS 3860 & 14.025 & 1.262 & 3532 & - & $3532 \pm 26$ & $\mathrm{dM} 2.5$ & $22.4221 \pm 0.0460$ & $10.778 \pm 0.024$ & $0.345 \pm 0.020$ & & & \\
\hline LHS 3879 & 12.868 & 1.450 & 3388 & 3428 & $3408 \pm 20$ & $\mathrm{sdM} 3.2$ & $43.0339 \pm 0.0480$ & $11.037 \pm 0.022$ & $0.377 \pm 0.025$ & -0.71 & 1 & \\
\hline LHS 3881 & 14.49 & 1.450 & 3388 & - & $3388 \pm 26$ & dM3.3 & $23.3948 \pm 0.0409$ & $11.336 \pm 0.024$ & $0.341 \pm 0.028$ & +0.05 & & \\
\hline LHS 3898 & 12.338 & 1.254 & 3539 & 3696 & $3617 \pm 79$ & $\mathrm{dM} 2.0$ & $34.4921 \pm 0.0546$ & $10.027 \pm 0.023$ & $0.442 \pm 0.055$ & +0.16 & & \\
\hline LHS 3903 & 14.55 & 1.351 & 3464 & - & $3464 \pm 26$ & $\mathrm{dM} 2.9$ & $18.6857 \pm 0.0385$ & $10.908 \pm 0.024$ & $0.361 \pm 0.028$ & & & \\
\hline NLTT 9223 & 12.688 & 1.429 & 3406 & 3377 & $3391 \pm 14$ & $\mathrm{dM} 3.3$ & $52.0770 \pm 0.6965$ & $11.271 \pm 0.049$ & $0.349 \pm 0.024$ & & & \\
\hline LSPM J0355+5214 & 14.508 & 1.318 & 3486 & 3435 & $3460 \pm 25$ & $\mathrm{dM} 2.9$ & $25.1365 \pm 0.0383$ & $11.510 \pm 0.023$ & $0.276 \pm 0.021$ & & & Subdwarf? \\
\hline LSPM J1804+1354 & 13.227 & 1.384 & 3441 & 3375 & $3408 \pm 33$ & $\mathrm{dM} 3.2$ & $52.1520 \pm 0.0410$ & $11.813 \pm 0.022$ & $0.264 \pm 0.025$ & & & Subdwarf? \\
\hline LDS $2332 \mathrm{~B}$ & 13.76 & 1.363 & 3456 & - & $3456 \pm 26$ & $\mathrm{dM} 3.0$ & $25.3188 \pm 0.0338$ & $10.777 \pm 0.023$ & $0.389 \pm 0.030$ & & & \\
\hline HIP $14101 \mathrm{~A}$ & 10.54 & 1.475 & 3366 & 3359 & $3363 \pm 4$ & $\mathrm{dM} 3.5$ & $148.5466 \pm 0.0800$ & $12.152 \pm 0.021$ & $0.245 \pm 0.005$ & -0.28 & & Subdwarf? \\
\hline HIP $14101 \mathrm{~B}$ & 10.54 & 1.475 & 3366 & 3359 & $3363 \pm 4$ & $\mathrm{dM} 3.5$ & $148.5466 \pm 0.0800$ & $12.152 \pm 0.021$ & $0.245 \pm 0.005$ & -0.28 & & Subdwarf? \\
\hline HIP 28035 & 10.762 & 1.362 & 3457 & 3663 & $3560 \pm 103$ & $\mathrm{dM} 2.4$ & $71.2290 \pm 0.0324$ & $10.025 \pm 0.021$ & $0.473 \pm 0.075$ & & & \\
\hline HIP $28940 \mathrm{~B}$ & 15.08 & 1.363 & 3456 & - & $3456 \pm 26$ & $\mathrm{dM} 3.0$ & $17.1457 \pm 0.0529$ & $11.251 \pm 0.027$ & $0.313 \pm 0.025$ & & 3 & \\
\hline HIP $28940 \mathrm{C}$ & 15.08 & 1.363 & 3456 & - & $3456 \pm 26$ & $\mathrm{dM} 3.0$ & $17.1457 \pm 0.0529$ & $11.251 \pm 0.027$ & $0.313 \pm 0.025$ & & 3 & \\
\hline HIP 110893A & 10.333 & 1.468 & 3372 & 3391 & $3382 \pm 9$ & $\mathrm{dM} 3.3$ & $249.3926 \pm 0.1653$ & $12.317 \pm 0.021$ & $0.219 \pm 0.007$ & -0.12 & & Subdwarf? \\
\hline HIP $110893 \mathrm{~B}$ & 10.333 & 1.468 & 3372 & 3391 & $3382 \pm 9$ & $\mathrm{dM} 3.3$ & $249.9668 \pm 0.7414$ & $12.322 \pm 0.026$ & $0.219 \pm 0.007$ & -0.12 & & Subdwarf? \\
\hline G $14-42$ & 14.45 & 1.285 & 3512 & - & $3512 \pm 26$ & $\mathrm{dM} 2.7$ & $19.7944 \pm 0.0719$ & $10.933 \pm 0.028$ & $0.329 \pm 0.020$ & & & \\
\hline G $65-4$ & 14.475 & 1.438 & 3398 & - & $3398 \pm 26$ & dM3.3 & $18.6136 \pm 0.0481$ & $10.824 \pm 0.026$ & $0.424 \pm 0.034$ & & & \\
\hline G $131-5$ & 14.59 & 1.450 & 3388 & - & $3388 \pm 26$ & $\mathrm{dM} 3.3$ & $15.8756 \pm 0.0817$ & $10.594 \pm 0.031$ & $0.480 \pm 0.040$ & & & \\
\hline G $132-51 B$ & 13.01 & 1.438 & 3398 & - & $3398 \pm 26$ & $\mathrm{dM} 3.3$ & $32.7415 \pm 0.1185$ & $10.585 \pm 0.028$ & $0.473 \pm 0.039$ & +0.16 & 1 & \\
\hline G $132-72$ & 14.35 & 1.450 & 3388 & - & $3388 \pm 26$ & dM3.3 & $23.4568 \pm 0.0889$ & $11.201 \pm 0.028$ & $0.363 \pm 0.030$ & & & \\
\hline G $137-17$ & 14.68 & 1.300 & 3499 & - & $3499 \pm 26$ & $\mathrm{dM} 2.7$ & $17.5728 \pm 0.0466$ & $10.904 \pm 0.026$ & $0.339 \pm 0.026$ & & & \\
\hline G $201-50$ & 14.63 & 1.462 & 3378 & 3390 & $3384 \pm 6$ & $\mathrm{dM} 3.3$ & $30.8208 \pm 0.0308$ & $12.074 \pm 0.022$ & $0.244 \pm 0.010$ & -0.05 & & Subdwarf? \\
\hline G $212-26$ & 13.42 & 1.376 & 3447 & - & $3447 \pm 26$ & $\mathrm{dM} 3.0$ & $26.3258 \pm 0.0280$ & $10.522 \pm 0.022$ & $0.445 \pm 0.035$ & & & \\
\hline G $212-27$ & 13.793 & 1.450 & 3388 & - & $3388 \pm 26$ & dM3.3 & $26.2789 \pm 0.0320$ & $10.891 \pm 0.023$ & $0.418 \pm 0.034$ & & & \\
\hline G $230-31$ & 12.961 & 1.328 & 3480 & 3559 & $3519 \pm 40$ & $\mathrm{dM} 2.6$ & $27.9648 \pm 0.0223$ & $10.194 \pm 0.022$ & $0.459 \pm 0.035$ & & & \\
\hline G $247-35$ & 12.71 & 1.275 & 3520 & - & $3520 \pm 26$ & $\mathrm{dM} 2.6$ & $35.5290 \pm 0.0353$ & $10.463 \pm 0.022$ & $0.405 \pm 0.023$ & +0.75 & 2 & \\
\hline $\mathrm{BD}+30397 \mathrm{~B}$ & 12.508 & 1.300 & 3499 & - & $3499 \pm 26$ & $\mathrm{dM} 2.7$ & $24.4490 \pm 0.0833$ & $9.449 \pm 0.027$ & $0.663 \pm 0.052$ & & 2 & Binary? \\
\hline $\mathrm{BD}+87$ 91B & 13.99 & 1.438 & 3398 & - & $3398 \pm 26$ & dM3.3 & $28.1121 \pm 0.1002$ & $11.234 \pm 0.028$ & $0.351 \pm 0.029$ & & & \\
\hline & & & & & dM4 sample & & & & & & & \\
\hline GJ & & & & & \pm & & \pm & \pm & \pm & & & \\
\hline $\mathrm{Gl}$ & & & & & \pm & & \pm & \pm & \pm & & & \\
\hline GJ $1001 \mathrm{~A}$ & 12.84 & 1.556 & 3297 & 3351 & $3324 \pm 27$ & $\mathrm{dM} 3.7$ & $81.2282 \pm 0.1139$ & $12.389 \pm 0.023$ & $0.237 \pm 0.020$ & & 15 & \\
\hline GJ $1005 \mathrm{AB}$ & 11.483 & 1.535 & 3315 & 3227 & $3271 \pm 44$ & dM3.9 & $200.53 \pm 9.41$ & $12.994 \pm 0.120$ & $0.198 \pm 0.035$ & -0.47 & & Subdwarf? \\
\hline GJ 1006A & 12.255 & 1.584 & 3273 & 3232 & $3252 \pm 20$ & $\mathrm{dM} 4.0$ & $65.1222 \pm 0.1007$ & $11.324 \pm 0.023$ & $0.444 \pm 0.033$ & & 4 & Binary? \\
\hline GJ $1006 \mathrm{~B}$ & 13.21 & 1.595 & 3263 & 3232 & $3247 \pm 15$ & $\mathrm{dM} 4.1$ & $65.2483 \pm 0.0838$ & $12.283 \pm 0.023$ & $0.288 \pm 0.018$ & & 4 & \\
\hline GJ $1010 \mathrm{~B}$ & 14.10 & 1.535 & 3315 & - & $3315 \pm 28$ & sdM3.7 & $52.2114 \pm 0.0518$ & $12.689 \pm 0.022$ & $0.210 \pm 0.018$ & +0.01 & & \\
\hline GJ 1011 & 14.145 & 1.619 & 3246 & - & $3246 \pm 28$ & $\mathrm{dM} 4.1$ & $60.0288 \pm 0.1002$ & $13.037 \pm 0.024$ & $0.204 \pm 0.019$ & & 1 & Subdwarf? \\
\hline GJ 1013 & 12.75 & 1.548 & 3304 & 3243 & $3273 \pm 30$ & dM3.9 & $70.9713 \pm 0.0530$ & $12.005 \pm 0.022$ & $0.311 \pm 0.029$ & -0.07 & 4 & \\
\hline GJ 1024 & 13.72 & 1.595 & 3263 & - & $3263 \pm 28$ & $\mathrm{dM} 4.0$ & $54.7079 \pm 0.0796$ & $12.410 \pm 0.023$ & $0.263 \pm 0.024$ & & 4 & \\
\hline GJ 1025 & 13.33 & 1.523 & 3325 & - & $3325 \pm 28$ & dM3.6 & $80.9235 \pm 0.0819$ & $12.870 \pm 0.022$ & $0.189 \pm 0.017$ & & 3 & Subdwarf? \\
\hline GJ 1034 & 15.05 & 1.613 & 3250 & 3035 & $3142 \pm 107$ & $\mathrm{dM} 4.5$ & $46.5863 \pm 0.0861$ & $13.391 \pm 0.024$ & $0.214 \pm 0.065$ & -0.38 & 3 & \\
\hline GJ 1045 & 14.47 & 1.672 & 3211 & - & $3211 \pm 28$ & $\mathrm{dM} 4.2$ & $45.4220 \pm 0.0820$ & $12.756 \pm 0.024$ & $0.248 \pm 0.023$ & +0.30 & & \\
\hline GJ 1065 & 12.79 & 1.535 & 3315 & - & $3315 \pm 28$ & dM3.7 & $100.5599 \pm 0.1050$ & $12.802 \pm 0.022$ & $0.199 \pm 0.018$ & +0.20 & 11 & Subdwarf? \\
\hline
\end{tabular}


Table 2:: continued.

\begin{tabular}{|c|c|c|c|c|c|c|c|c|c|c|c|c|}
\hline Star & $\begin{array}{c}\mathrm{V} \\
(\mathrm{mag})\end{array}$ & $\begin{array}{c}(\mathrm{R}-\mathrm{I})_{C} \\
(\mathrm{mag})\end{array}$ & $\begin{array}{c}T_{e f f}{ }^{1} \\
(\mathrm{~K})\end{array}$ & $\begin{array}{c}T_{e f f}^{2} \\
(\mathrm{~K})\end{array}$ & $T_{(\mathrm{K})}^{T_{\text {eff }} \pm 3 \sigma^{3}}$ & $\begin{array}{l}\text { Spect. } \\
\text { Type }\end{array}$ & $\begin{array}{c}\pi \\
(\mathrm{m} ")\end{array}$ & $\begin{array}{c}M_{V} \\
\text { (mag) }\end{array}$ & $\begin{array}{c}R_{\star} \\
\left(R_{\odot}\right)\end{array}$ & {$[\mathrm{M} / \mathrm{H}]$} & $\begin{array}{l}\text { Hi. Res. } \\
\text { Spectra }\end{array}$ & Comments \\
\hline GJ 1068 & 13.58 & 1.643 & 3231 & - & $3231 \pm 28$ & $\mathrm{dM} 4.1$ & $140.5559 \pm 0.0354$ & $14.319 \pm 0.021$ & $0.116 \pm 0.010$ & +0.06 & 18 & Subdwarf? \\
\hline GJ 1092 & 13.775 & 1.612 & 3251 & 3207 & $3229 \pm 22$ & $\mathrm{dM} 4.1$ & $68.8440 \pm 0.0763$ & $12.964 \pm 0.022$ & $0.218 \pm 0.017$ & -0.40 & & \\
\hline GJ 1105 & 12.01 & 1.535 & 3315 & 3213 & $3264 \pm 51$ & $\mathrm{dM} 4.0$ & $113.0897 \pm 0.0661$ & $12.277 \pm 0.021$ & $0.279 \pm 0.039$ & +0.24 & 1 & \\
\hline GJ $1122 \mathrm{~A}$ & 14.505 & 1.689 & 3200 & - & $3200 \pm 28$ & $\mathrm{dM} 4.3 \mathrm{e}$ & $51.6411 \pm 0.0882$ & $13.070 \pm 0.024$ & $0.219 \pm 0.022$ & +0.16 & & \\
\hline GJ 1129 & 12.60 & 1.619 & 3246 & 3220 & $3233 \pm 13$ & $\mathrm{dM} 4.1$ & $90.8249 \pm 0.0849$ & $12.391 \pm 0.022$ & $0.281 \pm 0.017$ & +0.05 & 11 & \\
\hline GJ 1134 & 12.965 & 1.654 & 3223 & 3129 & $3176 \pm 47$ & $\mathrm{dM} 4.4$ & $99.4498 \pm 0.0717$ & $12.953 \pm 0.022$ & $0.244 \pm 0.035$ & -0.16 & & \\
\hline GJ 1138 & 13.000 & 1.640 & 3233 & 3151 & $3192 \pm 41$ & $\mathrm{dM} 4.3$ & $103.1065 \pm 0.1841$ & $13.066 \pm 0.024$ & $0.224 \pm 0.029$ & -0.57 & & \\
\hline GJ 1148 & 11.852 & 1.582 & 3274 & 3231 & $3252 \pm 21$ & $\mathrm{dM} 4.0$ & $90.7623 \pm 0.0527$ & $11.642 \pm 0.021$ & $0.383 \pm 0.029$ & & & Binary? \\
\hline GJ 1157 & 13.625 & 1.649 & 3227 & - & $3227 \pm 28$ & $\mathrm{dM} 4.1$ & $63.1262 \pm 0.0718$ & $12.626 \pm 0.022$ & $0.256 \pm 0.023$ & +0.61 & & \\
\hline GJ 1174 & 12.763 & 1.533 & 3317 & 3211 & $3264 \pm 53$ & $\mathrm{dM} 4.0$ & $58.5132 \pm 0.1752$ & $11.599 \pm 0.026$ & $0.382 \pm 0.056$ & +0.09 & & Binary? \\
\hline GJ 1193 & 13.825 & 1.535 & 3315 & - & $3315 \pm 28$ & $\mathrm{dM} 3.7$ & $44.1535 \pm 0.0682$ & $12.050 \pm 0.023$ & $0.281 \pm 0.025$ & -0.09 & & \\
\hline GJ 1204 & 13.780 & 1.610 & 3252 & - & $3252 \pm 28$ & $\mathrm{dM} 4.0 \mathrm{e}$ & $66.0830 \pm 0.0551$ & $12.880 \pm 0.022$ & $0.217 \pm 0.019$ & +0.43 & ? & \\
\hline GJ 1207 & 12.315 & 1.587 & 3270 & 3221 & $3245 \pm 24$ & $\mathrm{dM} 4.1 \mathrm{e}$ & $115.0339 \pm 0.0823$ & $12.619 \pm 0.022$ & $0.248 \pm 0.020$ & -0.15 & 9 & \\
\hline GJ 1215 & 13.68 & 1.493 & 3351 & - & $3351 \pm 28$ & $\mathrm{dM} 3.5$ & $80.7994 \pm 0.1696$ & $13.217 \pm 0.025$ & $0.154 \pm 0.013$ & +0.07 & & Subdwarf? \\
\hline GJ 1216 & 14.410 & 1.572 & 3283 & - & $3283 \pm 28$ & dM3.9 & $55.8526 \pm 0.1018$ & $13.145 \pm 0.024$ & $0.181 \pm 0.016$ & -0.40 & & Subdwarf? \\
\hline GJ $1218 \mathrm{~A}$ & 13.483 & 1.548 & 3304 & 3281 & $3292 \pm 11$ & sdM3.8 & $60.7706 \pm 0.0716$ & $12.401 \pm 0.023$ & $0.250 \pm 0.013$ & -0.95 & & \\
\hline GJ $1218 \mathrm{~B}$ & 13.483 & 1.548 & 3304 & 3281 & $3292 \pm 11$ & sdM3.8 & $60.7706 \pm 0.0716$ & $12.401 \pm 0.023$ & $0.250 \pm 0.013$ & -0.95 & & \\
\hline GJ 1222 & 13.120 & 1.563 & 3291 & 3162 & $3226 \pm 64$ & $\mathrm{dM} 4.2$ & $62.2155 \pm 0.0639$ & $12.089 \pm 0.022$ & $0.328 \pm 0.056$ & +0.06 & 3 & \\
\hline GJ 1225 & 15.385 & 1.697 & 3195 & - & $3195 \pm 28$ & $\mathrm{sdM} 4.3$ & $52.9885 \pm 0.0441$ & $14.006 \pm 0.022$ & $0.144 \pm 0.014$ & -0.49 & & Subdwarf? \\
\hline GJ 1235 & 13.39 & 1.643 & 3231 & 3094 & $3162 \pm 68$ & $\mathrm{dM} 4.4$ & $94.1985 \pm 0.2407$ & $13.260 \pm 0.026$ & $0.218 \pm 0.043$ & +0.14 & 1 & \\
\hline GJ 1254 & 12.53 & 1.568 & 3287 & 3265 & $3276 \pm 11$ & $\mathrm{dM} 3.9$ & $62.4692 \pm 0.0418$ & $11.508 \pm 0.021$ & $0.389 \pm 0.021$ & +0.22 & 1 & Binary? \\
\hline GJ 1263 & 12.720 & 1.511 & 3336 & 3252 & $3294 \pm 42$ & $\mathrm{dM} 3.8$ & $80.7724 \pm 0.1102$ & $12.256 \pm 0.023$ & $0.266 \pm 0.032$ & +0.22 & 2 & \\
\hline GJ 1265 & 13.620 & 1.643 & 3231 & 3023 & $3127 \pm 104$ & $\mathrm{dM} 4.6$ & $97.5087 \pm 0.0711$ & $13.565 \pm 0.022$ & $0.204 \pm 0.060$ & -0.12 & 16 & \\
\hline GJ 1270 & 13.245 & 1.607 & 3254 & 3270 & $3262 \pm 12$ & $\mathrm{dM} 4.0$ & $71.2764 \pm 0.1177$ & $12.510 \pm 0.024$ & $0.252 \pm 0.014$ & +0.04 & 1 & \\
\hline GJ 1289 & 12.645 & 1.654 & 3223 & 3164 & $3193 \pm 29$ & $\mathrm{dM} 4.4$ & $119.7106 \pm 0.1165$ & $13.036 \pm 0.022$ & $0.226 \pm 0.023$ & +0.12 & 1 & \\
\hline GJ 2036B & 13.92 & 1.625 & 3242 & 3247 & $3244 \pm 3$ & $\mathrm{sdM} 4.1 \mathrm{e}$ & $90.1653 \pm 0.0379$ & $13.695 \pm 0.021$ & $0.151 \pm 0.006$ & & & Subdwarf? \\
\hline GJ 2043B & 14.675 & 1.619 & 3246 & 3130 & $3188 \pm 42$ & $\mathrm{dM} 4.3$ & $58.0811 \pm 0.1182$ & $13.495 \pm 0.024$ & $0.185 \pm 0.025$ & +0.34 & & Subdwarf? \\
\hline GJ $2069 \mathrm{~A}$ & 11.97 & 1.595 & 3263 & 3231 & $3247 \pm 16$ & $\mathrm{dM} 4.1 \mathrm{e}$ & $62.0284 \pm 0.2144$ & $10.933 \pm 0.027$ & $0.536 \pm 0.036$ & +0.27 & 1 & PMS? \\
\hline GJ 3049 & 11.51 & 1.678 & 3208 & - & $3208 \pm 28$ & $\mathrm{dM} 4.2$ & $97.7275 \pm 0.0819$ & $11.460 \pm 0.022$ & $0.453 \pm 0.041$ & & 6 & Binary? \\
\hline GJ $3078 B$ & 13.905 & 1.582 & 3274 & - & $3274 \pm 28$ & dM3.9 & $64.6265 \pm 0.1019$ & $12.957 \pm 0.023$ & $0.200 \pm 0.018$ & +0.05 & & Subdwarf? \\
\hline GJ 3092B & 14.85 & 1.769 & 3148 & - & $3148 \pm 28$ & $\mathrm{dM} 4.5$ & $32.4362 \pm 0.0589$ & $12.405 \pm 0.073$ & $0.333 \pm 0.033$ & +0.05 & & \\
\hline GJ 3105 & 13.977 & 1.674 & 3210 & 3269 & $3239 \pm 30$ & $\mathrm{dM} 4.1$ & $40.1835 \pm 0.3172$ & $11.997 \pm 0.037$ & $0.334 \pm 0.034$ & & & \\
\hline GJ 3132 & 13.848 & 1.554 & 3299 & - & $3299 \pm 28$ & $\mathrm{dM} 3.8$ & $54.3863 \pm 0.0723$ & $12.525 \pm 0.023$ & $0.233 \pm 0.021$ & & 1 & \\
\hline GJ 3142 & 13.600 & 1.625 & 3242 & 3257 & $3250 \pm 7$ & $\mathrm{dM} 4.0 \mathrm{e}$ & $65.4462 \pm 0.0658$ & $12.679 \pm 0.022$ & $0.239 \pm 0.011$ & +0.12 & 6 & \\
\hline GJ 3149B & 13.125 & 1.595 & 3263 & 3239 & $3251 \pm 12$ & $\mathrm{dM} 4.0 \mathrm{e}$ & $74.4161 \pm 0.0799$ & $12.483 \pm 0.022$ & $0.261 \pm 0.015$ & +0.09 & & \\
\hline GJ 3150 & 14.070 & 1.617 & 3248 & 3216 & $3232 \pm 16$ & $\mathrm{dM} 4.1$ & $44.1724 \pm 0.0609$ & $12.296 \pm 0.023$ & $0.295 \pm 0.019$ & & & \\
\hline GJ 3198 & 12.35 & 1.508 & 3338 & 3447 & $3392 \pm 54$ & $\mathrm{dM} 3.3$ & $58.5094 \pm 0.0760$ & $11.186 \pm 0.023$ & $0.363 \pm 0.050$ & +0.07 & 1 & Binary? \\
\hline GJ 3235 & 13.010 & 1.550 & 3302 & 3256 & $3279 \pm 23$ & dM3.9 & $42.6657 \pm 0.5126$ & $11.160 \pm 0.046$ & $0.454 \pm 0.041$ & -0.04 & 4 & Binary? \\
\hline GJ $3240 \mathrm{~A}$ & 13.29 & 1.511 & 3335 & - & $3335 \pm 28$ & dM3.6 & $25.6350 \pm 0.0519$ & $10.334 \pm 0.024$ & $0.597 \pm 0.053$ & & 1 & \\
\hline GJ $3240 \mathrm{~A}$ & 13.29 & 1.511 & 3335 & - & $3335 \pm 28$ & $\mathrm{dM} 3.6$ & $25.6350 \pm 0.0519$ & $10.334 \pm 0.024$ & $0.597 \pm 0.053$ & & 1 & \\
\hline GJ 3263A & 13.13 & 1.506 & 3340 & 3290 & $3315 \pm 25$ & $\mathrm{dM} 3.7$ & $54.1068 \pm 0.1189$ & $11.796 \pm 0.025$ & $0.316 \pm 0.026$ & +0.09 & 19 & \\
\hline GJ 3263B & 13.13 & 1.506 & 3340 & 3290 & $3315 \pm 25$ & $\mathrm{dM} 3.7$ & $54.2433 \pm 0.1154$ & $11.802 \pm 0.025$ & $0.315 \pm 0.026$ & +0.09 & 19 & \\
\hline GJ $3283 B$ & 14.97 & 1.654 & 3223 & - & $3223 \pm 28$ & $\mathrm{dM} 4.2 \mathrm{e}$ & $28.8139 \pm 0.0557$ & $12.268 \pm 0.024$ & $0.304 \pm 0.028$ & & & \\
\hline GJ $3322 \mathrm{~A}$ & 12.278 & 1.572 & 3283 & 3348 & $3315 \pm 33$ & $\mathrm{dM} 3.7 \mathrm{e}$ & $41.9242 \pm 0.0822$ & $10.390 \pm 0.024$ & $0.604 \pm 0.060$ & +0.03 & 1 & PMS? \\
\hline GJ 3322B & 12.278 & 1.572 & 3283 & 3348 & $3315 \pm 33$ & $\mathrm{dM} 3.7 \mathrm{e}$ & $42.2441 \pm 0.2394$ & $10.407 \pm 0.032$ & $0.600 \pm 0.062$ & +0.03 & 1 & PMS? \\
\hline GJ $3343 \mathrm{~A}$ & 13.45 & 1.511 & 3335 & - & $3335 \pm 28$ & dM3.6 & $36.1605 \pm 0.3018$ & $11.241 \pm 0.038$ & $0.393 \pm 0.037$ & & 1 & Binary? \\
\hline GJ $3372 B$ & 13.565 & 1.654 & 3223 & - & $3223 \pm 28$ & $\mathrm{dM} 4.2 \mathrm{e}$ & $74.0334 \pm 0.0635$ & $12.912 \pm 0.022$ & $0.226 \pm 0.021$ & +0.15 & 1 & \\
\hline GJ $3374 \mathrm{~B}$ & 13.335 & 1.517 & 3330 & 3293 & $3312 \pm 9$ & dM3.7 & $50.3718 \pm 0.0465$ & $11.846 \pm 0.022$ & $0.311 \pm 0.015$ & & 1 & \\
\hline GJ 3378 & 11.689 & 1.575 & 3281 & 3283 & $3282 \pm 1$ & dM3.9 & $129.4589 \pm 0.0799$ & $12.250 \pm 0.021$ & $0.273 \pm 0.009$ & & & \\
\hline GJ 3379 & 11.281 & 1.633 & 3237 & 3216 & $3226 \pm 10$ & $\mathrm{dM} 4.2$ & $192.0675 \pm 0.0715$ & $12.698 \pm 0.021$ & $0.248 \pm 0.013$ & & & \\
\hline GJ 3386C & 13.13 & 1.535 & 3315 & - & $3315 \pm 28$ & dM3.7 & $36.7439 \pm 0.0304$ & $10.956 \pm 0.022$ & $0.466 \pm 0.041$ & +0.24 & 1 & Binary? \\
\hline GJ 3398 & 14.22 & 1.643 & 3231 & - & $3231 \pm 28$ & $\mathrm{dM} 4.1$ & $56.5741 \pm 0.1107$ & $12.983 \pm 0.024$ & $0.215 \pm 0.020$ & & & \\
\hline GJ 3405B & 13.33 & 1.548 & 3304 & - & $3304 \pm 28$ & $\mathrm{dM} 3.8$ & $65.9903 \pm 0.1111$ & $12.427 \pm 0.024$ & $0.242 \pm 0.022$ & +0.12 & 4 & \\
\hline
\end{tabular}


Table 2:: continued.

\begin{tabular}{|c|c|c|c|c|c|c|c|c|c|c|c|c|}
\hline Star & $\underset{(\mathrm{mag})}{\mathrm{V}}$ & $\begin{array}{c}(\mathrm{R}-\mathrm{I})_{C} \\
(\mathrm{mag})\end{array}$ & $\begin{array}{c}T_{\text {eff }}{ }^{1} \\
(\mathrm{~K})\end{array}$ & $\begin{array}{c}T_{e f f}^{2} \\
(\mathrm{~K})\end{array}$ & $T_{\text {eff }} \pm 3 \sigma^{3}$ & $\begin{array}{l}\text { Spect. } \\
\text { Type }\end{array}$ & $\begin{array}{c}\pi \\
(\mathrm{m} ")\end{array}$ & $\begin{array}{c}M_{V} \\
(\mathrm{mag})\end{array}$ & $\begin{array}{c}R_{\star} \\
\left(R_{\odot}\right)\end{array}$ & {$[\mathrm{M} / \mathrm{H}]$} & $\begin{array}{l}\text { Hi. Res. } \\
\text { Spectra }\end{array}$ & Comments \\
\hline GJ 3442 & 13.65 & 1.698 & 3194 & - & $3194 \pm 28$ & $\mathrm{dM} 4.3$ & 86. \pm 13 . & $13.322 \pm 0.326$ & $0.198 \pm 0.052$ & & 7 & Subdwarf? \\
\hline GJ 3466 & 12.85 & 1.678 & 3208 & 3100 & $3154 \pm 54$ & $\mathrm{dM} 4.5$ & $80.3526 \pm 0.8245$ & $12.375 \pm 0.042$ & $0.333 \pm 0.058$ & +0.09 & & \\
\hline GJ $3522 \mathrm{~A}$ & $10.895 ?$ & 1.607 & 3254 & 3182 & $3218 \pm 36$ & $\mathrm{dM} 4.2 \mathrm{e}$ & $224 . \pm 36$ & $12.646 \pm 0.344$ & $0.258 \pm 0.074$ & -0.01 & 5 & \\
\hline GJ $3522 \mathrm{~B}$ & $10.895 ?$ & 1.607 & 3254 & 3182 & $3218 \pm 36$ & $\mathrm{dM} 4.2 \mathrm{e}$ & 224. \pm 36 & $12.646 \pm 0.344$ & $0.258 \pm 0.074$ & -0.01 & 5 & \\
\hline GJ 3573 & 14.2 & 1.674 & 3210 & - & $3210 \pm 28$ & $\mathrm{dM} 4.2$ & $54.4900 \pm 0.0855$ & $12.882 \pm 0.203$ & $0.235 \pm 0.044$ & +0.25 & & \\
\hline GJ $3577 \mathrm{~A}$ & 13.89 & 1.548 & 3304 & 3500 & $3402 \pm 98$ & $\mathrm{dM} 3.3 \mathrm{e}$ & $36.6872 \pm 0.0680$ & $11.713 \pm 0.024$ & $0.279 \pm 0.066$ & +0.19 & & \\
\hline GJ $3577 \mathrm{~B}$ & 14.16 & 1.572 & 3283 & - & $3283 \pm 28$ & $\mathrm{dM} 3.9 \mathrm{e}$ & $36.7820 \pm 0.0523$ & $11.988 \pm 0.023$ & $0.308 \pm 0.028$ & +0.19 & & \\
\hline GJ 3599 & 13.180 & 1.537 & 3313 & - & $3313 \pm 28$ & dM3.7 & $46.7032 \pm 0.0548$ & $11.527 \pm 0.023$ & $0.359 \pm 0.032$ & +0.30 & & Binary? \\
\hline GJ 3612 & 11.940 & 1.502 & 3343 & 3390 & $3367 \pm 23$ & $\mathrm{dM} 3.4$ & $77.3442 \pm 0.2883$ & $11.382 \pm 0.028$ & $0.347 \pm 0.027$ & & 1 & \\
\hline GJ $3631 \mathrm{~A}$ & 15.365 & 1.619 & 3246 & 3039 & $3142 \pm 103$ & $\mathrm{sdM} 4.5 \mathrm{e}$ & $71.7830 \pm 0.0903$ & $14.645 \pm 0.023$ & $0.120 \pm 0.035$ & -0.64 & & \\
\hline GJ 3631B & 15.365 & 1.619 & 3246 & 3039 & $3142 \pm 103$ & $\mathrm{sdM} 4.5 \mathrm{e}$ & $71.7830 \pm 0.0903$ & $14.645 \pm 0.023$ & $0.120 \pm 0.035$ & -0.64 & & \\
\hline GJ 3637 & 14.055 & 1.619 & 3246 & - & $3246 \pm 28$ & $\mathrm{dM} 4.1$ & $71.9 \pm 3.1$ & $13.339 \pm 0.112$ & $0.177 \pm 0.024$ & -0.30 & & Subdwarf? \\
\hline GJ 3666 & 12.56 & 1.523 & 3325 & 3243 & $3284 \pm 41$ & $\mathrm{dM} 3.9$ & $78.0880 \pm 0.1359$ & $12.023 \pm 0.024$ & $0.302 \pm 0.036$ & -0.07 & 2 & \\
\hline GJ 3707 & 12.120 & 1.584 & 3273 & 3186 & $3229 \pm 43$ & $\mathrm{dM} 4.1$ & $81.5516 \pm 0.1043$ & $11.677 \pm 0.023$ & $0.394 \pm 0.049$ & +0.35 & 16 & Binary? \\
\hline GJ $3731 \mathrm{~A}$ & 12.70 & 1.521 & 3327 & - & $3327 \pm 28$ & dM3.6 & $47.1483 \pm 0.0896$ & $11.067 \pm 0.024$ & $0.432 \pm 0.038$ & & 1 & Binary? \\
\hline GJ 3764 & 12.86 & 1.493 & 3351 & 3473 & $3412 \pm 61$ & $\mathrm{dM} 3.2$ & $59.9101 \pm 0.0475$ & $11.747 \pm 0.022$ & $0.270 \pm 0.041$ & -0.09 & & \\
\hline GJ 3779 & 12.93 & 1.578 & 3278 & 3216 & $3247 \pm 31$ & $\mathrm{dM} 4.1$ & $72.7352 \pm 0.0611$ & $12.239 \pm 0.022$ & $0.294 \pm 0.028$ & -0.26 & & \\
\hline GJ 3780 & 12.895 & 1.566 & 3288 & 3320 & $3304 \pm 16$ & $\mathrm{dM} 3.8 \mathrm{e}$ & $70.8096 \pm 0.0707$ & $12.145 \pm 0.022$ & $0.275 \pm 0.017$ & -0.21 & & \\
\hline GJ 3789 & 12.105 & 1.595 & 3263 & 3257 & $3260 \pm 3$ & $\mathrm{dM} 4.0 \mathrm{e}$ & $54.6875 \pm 0.3313$ & $10.794 \pm 0.033$ & $0.557 \pm 0.024$ & +0.07 & & Binary? \\
\hline GJ 3800 & 13.5 & 1.666 & 3216 & 3265 & $3240 \pm 25$ & $\mathrm{dM} 4.1 \mathrm{e}$ & $47.4764 \pm 0.5164$ & $11.882 \pm 0.223$ & $0.351 \pm 0.066$ & +0.10 & & \\
\hline GJ 3801 & 11.97 & 1.511 & 3336 & 3254 & $3295 \pm 41$ & $\mathrm{dM} 3.8$ & $108.8253 \pm 0.0442$ & $12.154 \pm 0.021$ & $0.279 \pm 0.032$ & +0.14 & 2 & \\
\hline GJ 3804 & 11.872 & 1.497 & 3348 & 3283 & $3315 \pm 32$ & $\mathrm{dM} 3.7$ & $89.2264 \pm 0.0781$ & $11.624 \pm 0.022$ & $0.342 \pm 0.033$ & +0.09 & 22 & \\
\hline GJ $3832 \mathrm{C}$ & 13.95 & 1.572 & 3283 & - & $3283 \pm 28$ & $\mathrm{dM} 3.9 \mathrm{e}$ & $34.9052 \pm 0.0628$ & $11.664 \pm 0.024$ & $0.357 \pm 0.032$ & +0.59 & & \\
\hline GJ 3839 & 13.129 & 1.653 & 3224 & 3205 & $3215 \pm 5$ & $\mathrm{dM} 4.2$ & $60.00 \pm 2.20$ & $12.020 \pm 0.098$ & $0.346 \pm 0.028$ & & & \\
\hline GJ 3843 & 12.995 & 1.491 & 3353 & 3283 & $3318 \pm 35$ & dM3.7 & $74.5644 \pm 0.0808$ & $12.358 \pm 0.022$ & $0.243 \pm 0.025$ & -0.02 & & \\
\hline GJ 3873 & 12.355 & 1.585 & 3272 & 3288 & $3280 \pm 8$ & dM3.9 & $67.0722 \pm 0.0487$ & $11.488 \pm 0.022$ & $0.390 \pm 0.018$ & +0.11 & & Binary? \\
\hline GJ 3900 & 12.840 & 1.572 & 3283 & 3300 & $3286 \pm 4$ & dM3.9 & $46.8543 \pm 0.7673$ & $11.194 \pm 0.055$ & $0.441 \pm 0.024$ & +0.46 & 7 & Binary? \\
\hline GJ 3907 & 14.175 & 1.641 & 3232 & - & $3232 \pm 28$ & $\mathrm{dM} 4.1$ & $50.4856 \pm 0.4258$ & $12.691 \pm 0.038$ & $0.246 \pm 0.024$ & +0.06 & & \\
\hline GJ 3908 & 13.70 & 1.500 & 3345 & - & $3345 \pm 28$ & dM3.6 & $32.4545 \pm 0.0684$ & $11.256 \pm 0.025$ & $0.383 \pm 0.034$ & +0.04 & & Binary? \\
\hline GJ 3919 & 13.185 & 1.588 & 3269 & 3238 & $3253 \pm 15$ & $\mathrm{dM} 4.0$ & $61.2109 \pm 0.3184$ & $12.119 \pm 0.031$ & $0.307 \pm 0.021$ & +0.17 & & \\
\hline GJ 3991 & 11.855 & 1.663 & 3217 & 3233 & $3225 \pm 8$ & $\mathrm{dM} 4.2$ & $134.5971 \pm 0.4894$ & $12.500 \pm 0.028$ & $0.272 \pm 0.014$ & & & \\
\hline GJ 4020B & 14.70 & 1.631 & 3239 & - & $3239 \pm 28$ & $\mathrm{dM} 4.1 \mathrm{e}$ & $30.9605 \pm 0.0406$ & $12.154 \pm 0.023$ & $0.310 \pm 0.028$ & & & \\
\hline GJ 4030 & 13.520 & 1.515 & 3332 & - & $3332 \pm 28$ & dM3.6 & $46.8068 \pm 0.0402$ & $11.872 \pm 0.022$ & $0.296 \pm 0.026$ & -0.04 & & \\
\hline GJ 4049B & 13.530 & 1.572 & 3283 & - & $3283 \pm 28$ & dM3.9 & $88.0473 \pm 0.0484$ & $13.254 \pm 0.021$ & $0.172 \pm 0.015$ & -0.13 & 1 & Subdwarf? \\
\hline GJ 4063A & 12.195 & 1.535 & 3315 & 3304 & $3309 \pm 5$ & sdM3.7 & $91.8545 \pm 0.0493$ & $12.011 \pm 0.021$ & $0.290 \pm 0.012$ & -0.54 & 4 & \\
\hline GJ 4063B & 12.195 & 1.535 & 3315 & 3304 & $3309 \pm 5$ & sdM3.7 & $91.8545 \pm 0.0493$ & $12.011 \pm 0.021$ & $0.290 \pm 0.012$ & -0.54 & 4 & \\
\hline GJ 4065 & 12.477 & 1.599 & 3260 & 3227 & $3243 \pm 16$ & $\mathrm{dM} 4.1$ & $83.0284 \pm 0.0832$ & $12.073 \pm 0.022$ & $0.320 \pm 0.021$ & & & \\
\hline GJ 4108 & 15.35 & 1.690 & 3200 & - & $3200 \pm 28$ & $\mathrm{dM} 4.3$ & $47.3972 \pm 0.0719$ & $13.729 \pm 0.023$ & $0.162 \pm 0.016$ & & & Subdwarf? \\
\hline GJ 4132 & 13.4 & 1.562 & 3292 & - & $3292 \pm 28$ & $\mathrm{dM} 3.8$ & $51.9363 \pm 0.0654$ & $11.977 \pm 0.203$ & $0.304 \pm 0.056$ & & 3 & \\
\hline GJ 4169 & 13.975 & 1.660 & 3219 & 3129 & $3174 \pm 45$ & $\mathrm{dM} 4.4$ & $77.8446 \pm 0.0668$ & $13.431 \pm 0.022$ & $0.196 \pm 0.027$ & +0.07 & & Subdwarf? \\
\hline GJ 4177BA & $? 13.12$ & 1.511 & 3336 & - & $3336 \pm 28$ & dM3.6 & $47.9730 \pm 0.7228$ & $11.525 \pm 0.052$ & $0.344 \pm 0.035$ & -0.03 & 1 & \\
\hline GJ 4177BB & $? 13.12$ & 1.511 & 3336 & - & $3336 \pm 28$ & dM3.6 & $47.9730 \pm 0.7228$ & $11.525 \pm 0.052$ & $0.344 \pm 0.035$ & -0.03 & 1 & \\
\hline GJ $4178 \mathrm{~A}$ & 13.9 & 1.497 & 3348 & - & $3348 \pm 28$ & $\mathrm{sdM} 3.5$ & $41.2951 \pm 0.1118$ & $11.979 \pm 0.206$ & $0.273 \pm 0.050$ & -1.65 & & \\
\hline GJ $4178 B$ & 13.9 & 1.497 & 3348 & - & $3348 \pm 28$ & sdM3.5 & $41.2951 \pm 0.1118$ & $11.979 \pm 0.206$ & $0.273 \pm 0.050$ & -1.65 & & \\
\hline GJ $4185 \mathrm{~A}$ & 13.43 & 1.548 & 3304 & - & $3304 \pm 28$ & $\mathrm{dM} 3.8 \mathrm{e}$ & $49.2145 \pm 0.2439$ & $11.890 \pm 0.031$ & $0.309 \pm 0.029$ & +0.18 & 1 & \\
\hline GJ $4185 B$ & 13.43 & 1.548 & 3304 & 3288 & $3296 \pm 8$ & $\mathrm{dM} 3.8$ & $49.2145 \pm 0.2439$ & $11.890 \pm 0.031$ & $0.314 \pm 0.016$ & +0.18 & 1 & \\
\hline GJ $4186 B$ & 13.49 & 1.560 & 3293 & 3288 & $3290 \pm 2$ & $\mathrm{dM} 3.8$ & $49.8995 \pm 0.0545$ & $11.980 \pm 0.022$ & $0.305 \pm 0.011$ & +0.18 & 1 & \\
\hline GJ 4207 & 12.57 & 1.505 & 3341 & 3340 & $3340 \pm 1$ & dM3.6 & $82.8819 \pm 0.1133$ & $12.162 \pm 0.023$ & $0.255 \pm 0.008$ & +0.01 & 1 & \\
\hline GJ $4214 \mathrm{~A}$ & 13.65 & 1.584 & 3273 & - & $3273 \pm 28$ & dM3.9 & $58.3917 \pm 0.1541$ & $12.482 \pm 0.026$ & $0.250 \pm 0.023$ & +0.21 & 1 & \\
\hline GJ 4232 & 14.06 & 1.517 & 3330 & - & $3330 \pm 28$ & dM3.6 & $40.2447 \pm 0.0563$ & $12.084 \pm 0.023$ & $0.269 \pm 0.024$ & -0.40 & & \\
\hline GJ 4257 & 13.747 & 1.567 & 3287 & - & $3287 \pm 28$ & dM3.8 & $56.6531 \pm 0.0912$ & $12.513 \pm 0.023$ & $0.240 \pm 0.021$ & & 1 & \\
\hline GJ 4269 & 13.971 & 1.622 & 3244 & 3248 & $3246 \pm 2$ & $\mathrm{dM} 4.1$ & $45.5231 \pm 0.0313$ & $12.262 \pm 0.021$ & $0.291 \pm 0.010$ & & & \\
\hline GJ $4277 \mathrm{~A}$ & 13.68 & 1.548 & 3304 & - & $3304 \pm 28$ & $\mathrm{dM} 3.8$ & $39.1 \pm 3.3$ & $11.641 \pm 0.196$ & $0.347 \pm 0.062$ & +0.24 & & \\
\hline
\end{tabular}


Table 2:: continued.

\begin{tabular}{|c|c|c|c|c|c|c|c|c|c|c|c|c|}
\hline Star & $\begin{array}{c}\mathrm{V} \\
(\mathrm{mag})\end{array}$ & $\begin{array}{c}(\mathrm{R}-\mathrm{I})_{C} \\
(\mathrm{mag})\end{array}$ & $\begin{array}{c}T_{\text {eff }}{ }^{1} \\
(\mathrm{~K})\end{array}$ & $\begin{array}{c}T_{\text {eff }}^{2} \\
(\mathrm{~K})\end{array}$ & $\begin{array}{c}T_{e f f} \pm 3 \sigma^{3} \\
(\mathrm{~K})\end{array}$ & $\begin{array}{l}\text { Spect. } \\
\text { Type }\end{array}$ & $\begin{array}{c}\pi \\
(\mathrm{m} ")\end{array}$ & $\begin{array}{c}M_{V} \\
(\mathrm{mag})\end{array}$ & $\begin{array}{c}R_{\star} \\
\left(R_{\odot}\right)\end{array}$ & {$[\mathrm{M} / \mathrm{H}]$} & $\begin{array}{l}\text { Hi. Res. } \\
\text { Spectra }\end{array}$ & Comments \\
\hline Gl 4285 & 14.45 & 1.560 & 3293 & - & $3293 \pm 28$ & dM3.8 & $35.3687 \pm 0.0510$ & $12.193 \pm 0.023$ & $0.275 \pm 0.024$ & & 12 & \\
\hline GJ 4333 & 11.710 & 1.601 & 3258 & 3225 & $3241 \pm 16$ & $\mathrm{dM} 4.1$ & $94.6489 \pm 0.0913$ & $11.591 \pm 0.022$ & $0.401 \pm 0.026$ & +0.25 & 12 & Binary? \\
\hline GJ 4338B & 12.435 & 1.619 & 3246 & 3257 & $3251 \pm 6$ & $\mathrm{dM} 4.0 \mathrm{e}$ & $67.5362 \pm 0.1868$ & $11.583 \pm 0.026$ & $0.395 \pm 0.018$ & & 1 & Binary? \\
\hline GJ 4367 & 13.45 & 1.617 & 3248 & 3221 & $3234 \pm 13$ & $\mathrm{dM} 4.1$ & $58.9687 \pm 0.1169$ & $12.303 \pm 0.024$ & $0.293 \pm 0.018$ & +0.28 & 3 & \\
\hline GJ 4376A & 13.794 & 1.508 & 3338 & - & $3338 \pm 28$ & dM3.6 & $25.2900 \pm 0.0757$ & $10.809 \pm 0.026$ & $0.477 \pm 0.043$ & & 1 & Binary? \\
\hline GJ $4378 \mathrm{~A}$ & 12.975 & 1.560 & 3293 & 3313 & $3303 \pm 10$ & $\mathrm{dM} 3.8$ & $50.1803 \pm 0.1883$ & $11.478 \pm 0.028$ & $0.375 \pm 0.020$ & & 2 & \\
\hline GJ 4387A & 13.56 & 1.542 & 3309 & 3255 & $3282 \pm 27$ & sdM3.9 & $63.6455 \pm 0.0914$ & $12.579 \pm 0.023$ & $0.235 \pm 0.016$ & -0.62 & & \\
\hline GJ 4387B & 13.56 & 1.542 & 3309 & 3255 & $3282 \pm 27$ & sdM3.9 & $63.6455 \pm 0.0914$ & $12.579 \pm 0.023$ & $0.235 \pm 0.016$ & -0.62 & & \\
\hline GJ 9652B & 13.275 & 1.542 & 3309 & 3356 & $3332 \pm 24$ & $\mathrm{dM} 3.6 \mathrm{e}$ & $55.2509 \pm 0.0567$ & $11.987 \pm 0.022$ & $0.280 \pm 0.022$ & -0.09 & 1 & \\
\hline Gl $15 \mathrm{~B}$ & 11.055 & 1.587 & 3270 & 3262 & $3266 \pm 4$ & sdM4.0 & $280.7866 \pm 0.0519$ & $13.297 \pm 0.020$ & $0.174 \pm 0.007$ & -0.95 & 5 & \\
\hline Gl 46 & 11.801 & 1.508 & 3338 & 3343 & $3340 \pm 3$ & dM3.6 & $83.5181 \pm 0.4599$ & $11.410 \pm 0.032$ & $0.360 \pm 0.015$ & +0.15 & 5 & \\
\hline Gl 53.1B & 14.054 & 1.675 & 3210 & 3257 & $3233 \pm 24$ & $\mathrm{dM} 4.1$ & $48.6871 \pm 0.0693$ & $12.491 \pm 0.023$ & $0.269 \pm 0.022$ & & & \\
\hline Gl 54.1 & 12.074 & 1.708 & 3188 & 3084 & $3136 \pm 52$ & $\mathrm{dM} 4.6 \mathrm{e}$ & $269.3628 \pm 0.0785$ & $14.226 \pm 0.021$ & $0.148 \pm 0.023$ & -0.33 & 107 & \\
\hline Gl 73 & 14.115 & 1.624 & 3243 & - & $3243 \pm 28$ & $\mathrm{dM} 4.1$ & $61.5492 \pm 0.0591$ & $13.061 \pm 0.022$ & $0.203 \pm 0.018$ & +0.15 & & \\
\hline Gl $84.1 \mathrm{~B}$ & 12.77 & 1.497 & 3348 & 3295 & $3321 \pm 26$ & $\mathrm{dM} 3.7$ & $38.1477 \pm 0.1017$ & $10.677 \pm 0.026$ & $0.523 \pm 0.045$ & -0.18 & 7 & Binary? \\
\hline Gl 101 & 13.213 & 1.499 & 3346 & - & $3346 \pm 28$ & $\mathrm{dM} 3.5$ & $49.8940 \pm 0.0450$ & $11.703 \pm 0.022$ & $0.311 \pm 0.027$ & & 1 & \\
\hline Gl 102 & 12.96 & 1.698 & 3194 & 3153 & $3173 \pm 20$ & $\mathrm{dM} 4.4 \mathrm{e}$ & $100.2835 \pm 0.1061$ & $12.966 \pm 0.022$ & $0.244 \pm 0.019$ & +0.03 & 3 & \\
\hline Gl 105B & 11.660 & 1.607 & 3254 & 3187 & $3220 \pm 33$ & $\mathrm{dM} 4.2$ & $138.4637 \pm 0.0886$ & $12.367 \pm 0.021$ & $0.292 \pm 0.030$ & -0.09 & 31 & \\
\hline Gl $118.2 \mathrm{C}$ & 13.827 & 1.600 & 3259 & 3227 & $3243 \pm 16$ & $\mathrm{dM} 4.1$ & $44.2923 \pm 0.0637$ & $12.059 \pm 0.023$ & $0.322 \pm 0.021$ & & & \\
\hline Gl 166C & 11.180 & 1.63 & 3239 & 3234 & $3236 \pm 2$ & $\mathrm{dM} 4.1 \mathrm{e}$ & $199.4552 \pm 0.3204$ & $12.679 \pm 0.023$ & $0.245 \pm 0.009$ & -0.10 & 5 & \\
\hline GL $169.1 \mathrm{~A}$ & 11.075 & 1.60 & 3259 & 3204 & $3231 \pm 27$ & $\mathrm{dM} 4.1$ & $180.4215 \pm 0.5863$ & $12.356 \pm 0.027$ & $0.287 \pm 0.026$ & +0.29 & 1 & \\
\hline GL 179 & 11.999 & 1.492 & 3352 & 3398 & $3375 \pm 23$ & $\mathrm{dM} 3.4$ & $80.9036 \pm 0.0606$ & $11.539 \pm 0.022$ & $0.318 \pm 0.024$ & +0.22 & 25 & \\
\hline Gl 206A & 12.282 & 1.510 & 3336 & 3248 & $3292 \pm 44$ & $\mathrm{dM} 3.8 \mathrm{e}$ & $77.1801 \pm 0.0813$ & $11.720 \pm 0.022$ & $0.342 \pm 0.042$ & +0.19 & 6 & \\
\hline Gl 206B & 12.282 & 1.510 & 3336 & 3248 & $3292 \pm 44$ & $\mathrm{dM} 3.8 \mathrm{e}$ & $77.1801 \pm 0.0813$ & $11.720 \pm 0.022$ & $0.342 \pm 0.042$ & +0.19 & 6 & \\
\hline GL 213 & 11.550 & 1.626 & 3242 & 3182 & $3212 \pm 30$ & $\mathrm{dM} 4.2 \mathrm{e}$ & $172.7068 \pm 0.0788$ & $12.737 \pm 0.021$ & $0.250 \pm 0.024$ & -0.16 & 67 & \\
\hline GL 232 & 13.06 & 1.679 & 3207 & 3103 & $3155 \pm 52$ & $\mathrm{dM} 4.5$ & $117.7410 \pm 0.0618$ & $13.415 \pm 0.021$ & $0.206 \pm 0.032$ & -0.25 & $?$ & \\
\hline GL $234 \mathrm{~A}$ & 11.12 & 1.700 & 3193 & - & $3193 \pm 28$ & $\mathrm{dM} 4.3 \mathrm{e}$ & $242.9649 \pm 0.8833$ & $13.048 \pm 0.028$ & $0.225 \pm 0.023$ & & 1 & \\
\hline GL 268A & 12.223 & 1.701 & 3192 & 3154 & $3173 \pm 19$ & $\mathrm{dM} 4.4 \mathrm{e}$ & $164.6395 \pm 0.1338$ & $13.306 \pm 0.022$ & $0.208 \pm 0.016$ & +0.16 & $?$ & \\
\hline GL $268 \mathrm{~B}$ & 12.223 & 1.701 & 3192 & 3154 & $3173 \pm 19$ & $\mathrm{dM} 4.4 \mathrm{e}$ & $164.6395 \pm 0.1338$ & $13.306 \pm 0.022$ & $0.208 \pm 0.016$ & +0.16 & $?$ & \\
\hline GL 273 & 9.850 & 1.559 & 3294 & 3277 & $3285 \pm 8$ & dM3.9 & $262.98 \pm 1.39$ & $11.950 \pm 0.031$ & $0.312 \pm 0.016$ & -0.08 & 196 & \\
\hline Gl 277B & 11.785 & 1.550 & 3302 & 3312 & $3307 \pm 5$ & $\mathrm{dM} 3.8 \mathrm{e}$ & $83.3267 \pm 0.0687$ & $11.389 \pm 0.022$ & $0.387 \pm 0.016$ & +0.10 & 4 & \\
\hline GL 285 & 11.225 & 1.696 & 3196 & 3166 & $3181 \pm 15$ & $\mathrm{dM} 4.4 \mathrm{e}$ & $167.0186 \pm 0.0592$ & $12.339 \pm 0.021$ & $0.320 \pm 0.021$ & +0.24 & 7 & \\
\hline GL 299 & 12.83 & 1.71 & 3187 & 3114 & $3150 \pm 36$ & $\mathrm{dM} 4.5$ & $145.4757 \pm 0.5695$ & $13.644 \pm 0.028$ & $0.187 \pm 0.023$ & -0.43 & 22 & Subdwarf? \\
\hline GL 300 & 12.090 & 1.68 & 3206 & 3189 & $3197 \pm 8$ & $\mathrm{dM} 4.3$ & $123.2204 \pm 0.0667$ & $12.543 \pm 0.021$ & $0.281 \pm 0.014$ & +0.20 & 40 & \\
\hline GL 317 & 11.990 & 1.497 & 3348 & 3262 & $3305 \pm 43$ & $\mathrm{dM} 3.8$ & $65.7744 \pm 0.0557$ & $11.080 \pm 0.022$ & $0.448 \pm 0.054$ & -0.10 & 84 & Binary? \\
\hline GL 319B & 11.83 & 1.523 & 3325 & 3555 & $3440 \pm 115$ & $\mathrm{dM} 3.1$ & $64.5835 \pm 0.0549$ & $10.881 \pm 0.022$ & $0.382 \pm 0.105$ & +0.04 & 9 & Binary? \\
\hline GL $324 \mathrm{~B}$ & 13.15 & 1.650 & 3226 & 3180 & $3203 \pm 23$ & $\mathrm{dM} 4.3$ & $80.1166 \pm 0.1091$ & $12.669 \pm 0.023$ & $0.262 \pm 0.021$ & +0.42 & & \\
\hline GL $347 \mathrm{~B}$ & 15.065 & 1.619 & 3246 & 3289 & $3267 \pm 21$ & sdM4.0 & $57.1832 \pm 0.0668$ & $13.851 \pm 0.023$ & $0.135 \pm 0.010$ & +0.02 & 1 & Subdwarf? \\
\hline GL $375 \mathrm{~A}$ & 12.053 & 1.501 & 3344 & 3307 & $3325 \pm 16$ & $\mathrm{dM} 3.7 \mathrm{e}$ & $64.7829 \pm 0.0448$ & $11.110 \pm 0.022$ & $0.426 \pm 0.026$ & & 1 & PMS? \\
\hline GL $375 B$ & 12.053 & 1.501 & 3344 & 3307 & $3325 \pm 18$ & $\mathrm{dM} 3.7 \mathrm{e}$ & $64.7829 \pm 0.0448$ & $11.110 \pm 0.022$ & $0.426 \pm 0.028$ & & 1 & PMS? \\
\hline GL 398 & 12.636 & 1.520 & 3328 & 3291 & $3309 \pm 16$ & $\mathrm{dM} 3.8 \mathrm{e}$ & $65.3811 \pm 0.0915$ & $11.713 \pm 0.023$ & $0.332 \pm 0.021$ & +0.03 & 7 & \\
\hline Gl 402 & 11.663 & 1.832 & 3099 & 3175 & $3137 \pm 38$ & $\mathrm{dM} 4.6 \mathrm{e}$ & $143.4971 \pm 0.0626$ & $12.447 \pm 0.021$ & $0.334 \pm 0.031$ & +0.12 & 11 & \\
\hline Gl 431 & 11.535 & 1.490 & 3354 & 3350 & $3352 \pm 2$ & $\mathrm{dM} 3.5 \mathrm{e}$ & $93.0715 \pm 0.0833$ & $11.379 \pm 0.022$ & $0.357 \pm 0.012$ & +1.24 & 5 & \\
\hline Gl 445 & 10.830 & 1.520 & 3328 & 3205 & $3266 \pm 61$ & $\mathrm{dM} 4.0$ & $190.2625 \pm 0.0475$ & $12.227 \pm 0.021$ & $0.285 \pm 0.046$ & -0.30 & 2 & \\
\hline Gl 447 & 11.160 & 1.697 & 3198 & 3105 & $3151 \pm 46$ & $\mathrm{dM} 4.5$ & $296.3073 \pm 0.0699$ & $13.519 \pm 0.021$ & $0.198 \pm 0.028$ & -0.04 & 112 & Subdwarf? \\
\hline Gl 452.1 & 12.775 & 1.489 & 3354 & 3284 & $3319 \pm 35$ & $\mathrm{dM} 3.7$ & $86.7442 \pm 0.0807$ & $12.466 \pm 0.022$ & $0.231 \pm 0.024$ & & 16 & \\
\hline GL $458 \mathrm{~B}$ & 13.33 & 1.523 & 3325 & 3320 & $3322 \pm 2$ & sdM3.7 & $65.5990 \pm 0.0372$ & $12.414 \pm 0.021$ & $0.235 \pm 0.008$ & -0.53 & & \\
\hline Gl 469 & 12.047 & 1.519 & 3329 & 3215 & $3272 \pm 57$ & dM3.9 & $68.6214 \pm 0.8950$ & $11.229 \pm 0.048$ & $0.446 \pm 0.074$ & +0.14 & 10 & Binary? \\
\hline GL 486 & 11.392 & 1.537 & 3313 & 3268 & $3290 \pm 22$ & $\mathrm{dM} 3.8$ & $123.8215 \pm 0.0623$ & $11.856 \pm 0.021$ & $0.323 \pm 0.024$ & -0.05 & 13 & \\
\hline GL $490 B$ & 13.180 & 1.595 & 3263 & 3182 & $3222 \pm 40$ & $\mathrm{dM} 4.2$ & $49.5475 \pm 0.1263$ & $11.655 \pm 0.026$ & $0.403 \pm 0.048$ & +0.07 & & Binary? \\
\hline GL 512B & 13.69 & 1.652 & 3225 & 3130 & $3177 \pm 47$ & $\mathrm{dM} 4.4$ & $71.9908 \pm 0.2344$ & $12.976 \pm 0.027$ & $0.240 \pm 0.035$ & +0.12 & 5 & \\
\hline GL $520 \mathrm{C}$ & 14.47 & 1.584 & 3273 & 3150 & $3211 \pm 61$ & $\mathrm{dM} 4.2 \mathrm{e}$ & $45.1562 \pm 0.0599$ & $12.744 \pm 0.023$ & $0.250 \pm 0.041$ & -0.14 & & \\
\hline GL 540.2 & 13.875 & 1.726 & 3176 & - & $3176 \pm 28$ & $\mathrm{sdM} 4.4 \mathrm{e}$ & $31.7829 \pm 0.0813$ & $11.386 \pm 0.026$ & $0.501 \pm 0.050$ & -0.80 & 12 & Binary? \\
\hline
\end{tabular}


Table 2:: continued.

\begin{tabular}{|c|c|c|c|c|c|c|c|c|c|c|c|c|}
\hline Star & $\underset{(\mathrm{mag})}{\mathrm{V}}$ & $\begin{array}{c}(\mathrm{R}-\mathrm{I})_{C} \\
(\mathrm{mag})\end{array}$ & $\begin{array}{c}T_{\text {eff }}{ }^{1} \\
(\mathrm{~K})\end{array}$ & $\begin{array}{c}T_{e f f}^{2} \\
(\mathrm{~K})\end{array}$ & $T_{\text {eff }} \pm 3 \sigma^{3}$ & $\begin{array}{l}\text { Spect. } \\
\text { Type }\end{array}$ & $\begin{array}{c}\pi \\
(\mathrm{m} ")\end{array}$ & $\begin{array}{c}M_{V} \\
(\mathrm{mag})\end{array}$ & $\begin{array}{c}R_{\star} \\
\left(R_{\odot}\right)\end{array}$ & {$[\mathrm{M} / \mathrm{H}]$} & $\begin{array}{l}\text { Hi. Res. } \\
\text { Spectra }\end{array}$ & Comments \\
\hline GL 544B & 14.50 & 1.651 & 3225 & 3191 & $3208 \pm 17$ & $\mathrm{dM} 4.2$ & $48.6039 \pm 0.0951$ & $12.933 \pm 0.024$ & $0.230 \pm 0.016$ & -0.13 & 5 & \\
\hline GL 545 & 12.855 & 1.528 & 3321 & 3249 & $3285 \pm 36$ & $\mathrm{dM} 3.8$ & $72.4350 \pm 0.0724$ & $12.155 \pm 0.022$ & $0.284 \pm 0.030$ & -0.12 & & \\
\hline Gl 553.1 & 11.903 & 1.488 & 3355 & 3246 & $3300 \pm 54$ & $\mathrm{dM} 3.8$ & $92.0697 \pm 0.1046$ & $11.724 \pm 0.022$ & $0.337 \pm 0.049$ & +0.26 & 11 & \\
\hline GL 555 & 11.318 & 1.629 & 3240 & 3205 & $3222 \pm 17$ & $\mathrm{dM} 4.2$ & $160.1141 \pm 0.1135$ & $12.340 \pm 0.022$ & $0.294 \pm 0.020$ & +0.20 & 16 & \\
\hline GL 568A & 12.425 & 1.538 & 3312 & 3260 & $3286 \pm 26$ & dM3.9 & $87.9350 \pm 0.1779$ & $12.146 \pm 0.024$ & $0.285 \pm 0.024$ & +0.45 & & \\
\hline GL $568 \mathrm{~B}$ & 12.425 & 1.538 & 3312 & 3260 & $3286 \pm 26$ & $\mathrm{dM} 3.9$ & $87.9350 \pm 0.1779$ & $12.146 \pm 0.024$ & $0.285 \pm 0.024$ & +0.45 & & \\
\hline Gl 590 & 12.72 & 1.61 & 3252 & 3103 & $3178 \pm 65$ & $\mathrm{dM} 4.4$ & $99.1979 \pm 0.0886$ & $12.703 \pm 0.022$ & $0.272 \pm 0.051$ & & 1 & \\
\hline GL 592 & 12.720 & 1.561 & 3293 & 3196 & $3244 \pm 48$ & $\mathrm{dM} 4.1$ & $71.6550 \pm 0.0804$ & $11.996 \pm 0.022$ & $0.331 \pm 0.044$ & +0.14 & 6 & \\
\hline GL 609 & 12.570 & 1.595 & 3263 & 3123 & $3193 \pm 70$ & $\mathrm{dM} 4.3$ & $99.9043 \pm 0.0594$ & $12.568 \pm 0.021$ & $0.281 \pm 0.056$ & -0.06 & 5 & \\
\hline Gl 628 & 10.084 & 1.529 & 3320 & 3298 & $3309 \pm 11$ & $\mathrm{dM} 3.7$ & $232.2095 \pm 0.0630$ & $11.913 \pm 0.021$ & $0.303 \pm 0.016$ & & & \\
\hline GL $630.1 \mathrm{AA}$ & $12.995 ?$ & 1.684 & 3204 & 3115 & $3159 \pm 44$ & $\mathrm{dM} 4.5 \mathrm{e}$ & $67.3400 \pm 0.0514$ & $12.136 \pm 0.022$ & $0.368 \pm 0.050$ & -0.34 & 1 & \\
\hline GL $630.1 \mathrm{AB}$ & $12.995 ?$ & 1.684 & 3204 & 3115 & $3159 \pm 44$ & $\mathrm{dM} 4.5 \mathrm{e}$ & $67.3400 \pm 0.0514$ & $12.136 \pm 0.022$ & $0.368 \pm 0.050$ & -0.34 & 1 & \\
\hline GL 643 & 11.759 & 1.546 & 3305 & 3304 & $3304 \pm 1$ & $\mathrm{dM} 3.8$ & $153.9189 \pm 0.1310$ & $12.695 \pm 0.022$ & $0.213 \pm 0.007$ & -0.21 & 41 & \\
\hline GL $644 \mathrm{~A}$ & 9.776 & 1.529 & 3320 & - & $3320 \pm 28$ & $\mathrm{dM} 3.7$ & $161.41 \pm 5.64$ & $10.816 \pm 0.095$ & $0.492 \pm 0.061$ & -0.21 & & PMS? \\
\hline GL $644 \mathrm{~B}$ & 9.776 & 1.529 & 3320 & - & $3320 \pm 28$ & dM3.7 & $161.41 \pm 5.64$ & $10.816 \pm 0.095$ & $0.492 \pm 0.061$ & -0.21 & 4 & PMS? \\
\hline Gl 669A & 11.36 & 1.530 & 3319 & 3343 & $3331 \pm 12$ & $\mathrm{dM} 3.6 \mathrm{e}$ & $92.9885 \pm 0.0498$ & $11.202 \pm 0.021$ & $0.403 \pm 0.022$ & +0.08 & 7 & PMS? \\
\hline GL 682 & 10.946 & 1.59 & 3268 & 3207 & $3237 \pm 30$ & $\mathrm{dM} 4.2$ & $199.7031 \pm 0.0832$ & $12.448 \pm 0.021$ & $0.272 \pm 0.026$ & +0.11 & 40 & \\
\hline GL 695B & 10.8 & 1.500 & 3345 & 3271 & $3308 \pm 37$ & $\mathrm{dM} 3.7$ & $119.7908 \pm 0.6586$ & $11.192 \pm 0.212$ & $0.423 \pm 0.088$ & +0.40 & 1 & Binary? \\
\hline Gl 699 & 9.511 & 1.592 & 3266 & 3244 & $3255 \pm 11$ & $\mathrm{dM} 4.0$ & $547.4506 \pm 0.2899$ & $13.203 \pm 0.021$ & $0.186 \pm 0.010$ & -0.40 & 69 & \\
\hline GL $720 B$ & 13.010 & 1.548 & 3304 & 3216 & $3260 \pm 44$ & $\mathrm{dM} 4.0$ & $64.2842 \pm 0.0406$ & $12.051 \pm 0.021$ & $0.313 \pm 0.039$ & -0.16 & 1 & \\
\hline GL $725 B$ & 9.69 & 1.51 & 3336 & 3310 & $3323 \pm 13$ & sdM3.7 & $283.8624 \pm 0.1065$ & $11.956 \pm 0.021$ & $0.289 \pm 0.016$ & -0.57 & 8 & \\
\hline GL 729 & 10.475 & 1.580 & 3276 & 3200 & $3238 \pm 38$ & $\mathrm{dM} 4.1 \mathrm{e}$ & $336.1228 \pm 0.0641$ & $13.107 \pm 0.020$ & $0.200 \pm 0.022$ & -0.14 & 22 & \\
\hline GL $732 \mathrm{~A}$ & 12.70 & 1.560 & 3293 & 3207 & $3250 \pm 43$ & $\mathrm{dM} 4.0 \mathrm{e}$ & $80.2529 \pm 0.0796$ & $12.222 \pm 0.022$ & $0.294 \pm 0.036$ & -0.01 & 3 & \\
\hline GL 754 & 12.23 & 1.687 & 3202 & - & $3202 \pm 28$ & $\mathrm{dM} 4.3$ & $169.0921 \pm 0.2165$ & $13.371 \pm 0.023$ & $0.190 \pm 0.018$ & -0.17 & 137 & Subdwarf? \\
\hline GL $766 \mathrm{~A}$ & 12.47 & 1.654 & 3223 & 3219 & $3221 \pm 2$ & $\mathrm{dM} 4.2$ & $94.0139 \pm 0.0804$ & $12.336 \pm 0.022$ & $0.295 \pm 0.011$ & +0.04 & & \\
\hline GL $766 \mathrm{~B}$ & 13.01 & 1.654 & 3223 & 3219 & $3221 \pm 2$ & $\mathrm{dM} 4.2$ & $93.9479 \pm 0.0756$ & $12.874 \pm 0.022$ & $0.231 \pm 0.008$ & +0.04 & & \\
\hline GL $781.1 \mathrm{~B}$ & 12.51 & 1.582 & 3274 & 3297 & $3285 \pm 12$ & PMS M3.9e & $67.9318 \pm 0.0837$ & $11.670 \pm 0.023$ & $0.355 \pm 0.020$ & -0.07 & 1 & \\
\hline GL $783.2 \mathrm{~B}$ & 13.94 & 1.618 & 3247 & 3182 & $3214 \pm 32$ & $\mathrm{dM} 4.2$ & $49.3808 \pm 0.0672$ & $12.408 \pm 0.023$ & $0.290 \pm 0.029$ & -0.15 & & \\
\hline Gl $784.2 \mathrm{~A}$ & 13.230 & 1.518 & 3330 & - & $3330 \pm 28$ & $\mathrm{dM} 3.6$ & $43.5842 \pm 0.0559$ & $11.427 \pm 0.023$ & $0.364 \pm 0.032$ & & 1 & Binary? \\
\hline GL $791.2 \mathrm{~A}$ & 13.04 & 1.672 & 3212 & 3160 & $3186 \pm 26$ & $\mathrm{dM} 4.3 \mathrm{e}$ & $133.8113 \pm 1.3869$ & $13.672 \pm 0.042$ & $0.171 \pm 0.018$ & +0.06 & 1 & Subdwarf? \\
\hline GL 792 & 13.485 & 1.559 & 3294 & - & $3294 \pm 28$ & $\mathrm{dM} 3.8$ & $68.7044 \pm 0.3940$ & $12.670 \pm 0.032$ & $0.220 \pm 0.021$ & -0.11 & & \\
\hline GL $810 \mathrm{~A}$ & 12.475 & 1.615 & 3249 & 3253 & $3251 \pm 2$ & $\mathrm{dM} 4.0$ & $80.2319 \pm 0.0881$ & $11.997 \pm 0.022$ & $0.326 \pm 0.012$ & +0.02 & 5 & \\
\hline GL $812 \mathrm{~A}$ & 11.916 & 1.530 & 3319 & 3399 & $3359 \pm 40$ & dM3.5e & $62.2541 \pm 0.0958$ & $10.887 \pm 0.023$ & $0.442 \pm 0.049$ & +0.12 & 12 & PMS? \\
\hline GL $838.1 \mathrm{~B}$ & 14.93 & 1.625 & 3242 & - & $3242 \pm 28$ & $\mathrm{dM} 4.1$ & $44.2645 \pm 0.0429$ & $13.160 \pm 0.022$ & $0.194 \pm 0.018$ & +0.05 & & Subdwarf? \\
\hline Gl $852 \mathrm{~A}$ & 13.385 & 1.635 & 3236 & - & $3236 \pm 28$ & $\mathrm{dM} 4.1$ & $89.8025 \pm 0.0911$ & $13.151 \pm 0.022$ & $0.197 \pm 0.018$ & & 1 & Subdwarf? \\
\hline Gl $865 \mathrm{~A}$ & 12.218 & 1.509 & 3337 & 3338 & $3338 \pm 1$ & $\mathrm{dM} 3.6 \mathrm{e}$ & $75.87 \pm 5.83$ & $11.618 \pm 0.166$ & $0.328 \pm 0.028$ & -0.10 & 2 & \\
\hline Gl 865B & 12.218 & 1.509 & 3337 & 3303 & $3320 \pm 17$ & $\mathrm{dM} 3.7 \mathrm{e}$ & $75.87 \pm 5.83$ & $11.599 \pm 0.181$ & $0.340 \pm 0.041$ & -0.10 & 2 & \\
\hline Gl 867B & 11.440 & 1.511 & 3336 & - & $3336 \pm 28$ & sdM3.6e & $113.1171 \pm 0.0728$ & $11.708 \pm 0.021$ & $0.316 \pm 0.027$ & -0.59 & 2 & \\
\hline GL $873 \mathrm{~A}$ & 10.26 & 1.523 & 3325 & 3315 & $3320 \pm 5$ & $\mathrm{dM} 3.7 \mathrm{e}$ & $198.0112 \pm 0.0380$ & $11.743 \pm 0.020$ & $0.321 \pm 0.013$ & -0.05 & & \\
\hline GL $876 \mathrm{~A}$ & 10.192 & 1.548 & 3304 & 3331 & $3317 \pm 14$ & $\mathrm{dM} 3.7$ & $213.8669 \pm 0.0758$ & $11.843 \pm 0.021$ & $0.308 \pm 0.018$ & +0.15 & 262 & \\
\hline Gl 896A & 10.180 & 1.485 & 3358 & 3346 & $3352 \pm 6$ & $\mathrm{dM} 3.5 \mathrm{e}$ & $159.7098 \pm 0.0827$ & $11.197 \pm 0.021$ & $0.388 \pm 0.011$ & +0.10 & & PMS? \\
\hline LHS 1655 & 15.32 & 1.562 & 3292 & - & $3292 \pm 28$ & $\mathrm{dM} 3.8$ & $27.1869 \pm 0.0862$ & $12.492 \pm 0.027$ & $0.240 \pm 0.022$ & +0.18 & & \\
\hline LHS 2795 & 15.0 & 1.612 & 3251 & - & $3251 \pm 28$ & $\mathrm{dM} 4.0$ & $31.2691 \pm 0.0551$ & $12.476 \pm 0.204$ & $0.262 \pm 0.048$ & -1.59 & & \\
\hline LHS 3279 & 14.04 & 1.567 & 3287 & - & $3287 \pm 28$ & $\mathrm{dM} 3.9 \mathrm{e}$ & $26.0668 \pm 0.0447$ & $11.120 \pm 0.024$ & $0.456 \pm 0.041$ & & & Binary? \\
\hline LDS 1751 & 15.71 & 1.560 & 3293 & - & $3293 \pm 28$ & $\mathrm{dM} 3.8$ & $23.3147 \pm 0.0413$ & $12.548 \pm 0.024$ & $0.233 \pm 0.021$ & & & \\
\hline MCC 85B & 11.01 & 1.572 & 3283 & - & $3283 \pm 28$ & $\mathrm{sdM} 3.9 \mathrm{e}$ & $280.7866 \pm 0.0519$ & $13.252 \pm 0.020$ & $0.172 \pm 0.015$ & -0.95 & 1 & \\
\hline MCC 475B & 14.88 & 1.511 & 3336 & - & $3336 \pm 28$ & $\mathrm{dM} 3.6$ & $33.3465 \pm 0.0831$ & $12.495 \pm 0.025$ & $0.220 \pm 0.020$ & +0.04 & & \\
\hline G $32-37$ & 14.12 & 1.523 & 3325 & - & $3325 \pm 28$ & $\mathrm{dM} 3.6$ & $34.8178 \pm 0.0907$ & $11.829 \pm 0.026$ & $0.306 \pm 0.027$ & +0.19 & 1 & \\
\hline G $32-38$ & 14.40 & 1.523 & 3325 & - & $3325 \pm 28$ & $\mathrm{dM} 3.6$ & $35.6094 \pm 0.1575$ & $12.158 \pm 0.030$ & $0.263 \pm 0.024$ & +0.19 & & \\
\hline G $97-52 B$ & 13.62 & 1.535 & 3315 & - & $3315 \pm 28$ & $\mathrm{dM} 3.7$ & $45.1508 \pm 0.0806$ & $11.893 \pm 0.024$ & $0.302 \pm 0.027$ & & & \\
\hline G $255-12$ & 15.705 & 1.560 & 3293 & - & $3293 \pm 28$ & $\mathrm{dM} 3.8$ & $23.3147 \pm 0.0413$ & $12.543 \pm 0.024$ & $0.234 \pm 0.021$ & +0.75 & & \\
\hline G $184-24$ & 13.731 & 1.675 & 3210 & 3271 & $3240 \pm 31$ & $\mathrm{dM} 4.1$ & $70.9655 \pm 0.0440$ & $12.986 \pm 0.021$ & $0.211 \pm 0.020$ & & & \\
\hline HIP 103039 & 11.460 & 1.618 & 3247 & 3277 & $3262 \pm 15$ & $\mathrm{dM} 4.0$ & $178.1243 \pm 0.0849$ & $12.714 \pm 0.021$ & $0.229 \pm 0.014$ & & & \\
\hline
\end{tabular}


Table 2:: continued.

\begin{tabular}{|c|c|c|c|c|c|c|c|c|c|c|c|c|}
\hline Star & $\begin{array}{c}\mathrm{V} \\
(\mathrm{mag})\end{array}$ & $\begin{array}{c}(\mathrm{R}-\mathrm{I})_{C} \\
(\mathrm{mag})\end{array}$ & $\begin{array}{c}T_{\text {eff }}{ }^{1} \\
(\mathrm{~K})\end{array}$ & $\begin{array}{c}T_{e f f}^{2} \\
(\mathrm{~K})\end{array}$ & $\begin{array}{c}T_{\text {eff }} \pm 3 \sigma^{3} \\
(\mathrm{~K})\end{array}$ & $\begin{array}{l}\text { Spect. } \\
\text { Type }\end{array}$ & $\begin{array}{c}\pi \\
(\mathrm{m} ")\end{array}$ & $\begin{array}{c}M_{V} \\
(\mathrm{mag})\end{array}$ & $\begin{array}{c}R_{\star} \\
\left(R_{\odot}\right)\end{array}$ & {$[\mathrm{M} / \mathrm{H}]$} & $\begin{array}{l}\text { Hi. Res. } \\
\text { Spectra }\end{array}$ & Comments \\
\hline HD 183870B & 13.894 & 1.626 & 3242 & 3211 & $3226 \pm 15$ & $\mathrm{dM} 4.2$ & $56.5564 \pm 0.0709$ & $12.656 \pm 0.023$ & $0.252 \pm 0.016$ & & & \\
\hline LP 99-392 & 13.426 & 1.572 & 3283 & 3252 & $3267 \pm 15$ & $\mathrm{dM} 4.0$ & $51.2843 \pm 0.0424$ & $11.976 \pm 0.022$ & $0.319 \pm 0.020$ & & & \\
\hline \multirow[t]{2}{*}{ LP $348-40$} & 11.958 & 1.527 & 3322 & 3358 & $3340 \pm 18$ & dM3.6 & $48.8609 \pm 0.0801$ & $10.403 \pm 0.024$ & $0.573 \pm 0.038$ & & & Binary? \\
\hline & & & & & dM5 sample & & & & & & & \\
\hline GJ 1014 & 15.32 & 1.867 & 3068 & - & $3068 \pm 32$ & $\mathrm{dM} 4.9$ & $69.4698 \pm 0.0731$ & $14.529 \pm 0.022$ & $0.148 \pm 0.017$ & -0.02 & - & \\
\hline GJ 1028 & 14.47 & 1.893 & 3046 & - & $3046 \pm 32$ & $\mathrm{dM} 5.0$ & $102.3224 \pm 0.0752$ & $14.520 \pm 0.022$ & $0.156 \pm 0.018$ & & - & \\
\hline GJ 1029 & 14.790 & 1.933 & 3011 & 2981 & $2996 \pm 15$ & dM5.3 & $79.8408 \pm 0.3404$ & $14.301 \pm 0.029$ & $0.192 \pm 0.016$ & -0.10 & - & \\
\hline GJ 1035 & 14.765 & 1.802 & 3125 & - & $3125 \pm 32$ & $\mathrm{dM} 4.6$ & $69.0676 \pm 0.0623$ & $13.961 \pm 0.022$ & $0.171 \pm 0.019$ & & - & \\
\hline GJ 1053 & 14.64 & 1.812 & 3116 & 2909 & $3012 \pm 103 \mathrm{~s}$ dM5.2 & $82.7034 \pm 0.0577$ & $14.228 \pm 0.022$ & $0.192 \pm 0.058$ & -0.50 & - & & \\
\hline GJ 1055 & 14.855 & 1.778 & 3142 & - & $3142 \pm 32$ & $\mathrm{dM} 4.5$ & $79.5076 \pm 0.0775$ & $14.357 \pm 0.022$ & $0.137 \pm 0.015$ & & - & \\
\hline GJ 1057 & 13.840 & 1.840 & 3092 & 3014 & $3053 \pm 39$ & $\mathrm{dM} 4.9(\mathrm{e})$ & $116.1536 \pm 0.0882$ & $14.165 \pm 0.022$ & $0.181 \pm 0.023$ & +0.08 & 16 & \\
\hline GJ 1059 & 15.325 & 1.845 & 3087 & 3048 & $3067 \pm 19$ & $\mathrm{dM} 4.9$ & $67.6990 \pm 0.0787$ & $14.478 \pm 0.023$ & $0.152 \pm 0.012$ & -0.36 & - & \\
\hline GJ $1103 \mathrm{~A}$ & 13.255 & 1.756 & 3156 & 3092 & $3124 \pm 32$ & $\mathrm{dM} 4.6$ & $107.7759 \pm 0.0917$ & $13.418 \pm 0.022$ & $0.220 \pm 0.024$ & +0.02 & - & \\
\hline GJ $1103 B$ & 14.70 & 1.756 & 3156 & 3089 & $3122 \pm 33$ & $\mathrm{dM} 4.6$ & $107.7759 \pm 0.0917$ & $14.863 \pm 0.022$ & $0.113 \pm 0.013$ & +0.02 & - & Subdwarf? \\
\hline GJ 1119 & 13.325 & 1.724 & 3177 & 3125 & $3151 \pm 26$ & $\mathrm{dM} 4.5 \mathrm{e}$ & $99.1286 \pm 0.0646$ & $13.306 \pm 0.021$ & $0.218 \pm 0.021$ & +0.05 & 2 & \\
\hline GJ $1122 B$ & 14.635 & 1.701 & 3192 & 3184 & $3188 \pm 4$ & $\mathrm{dM} 4.3$ & $51.7026 \pm 0.0703$ & $13.203 \pm 0.023$ & $0.212 \pm 0.009$ & +0.16 & - & \\
\hline Gl 1123 & 13.10 & 1.746 & 3163 & - & $3163 \pm 32$ & $\mathrm{dM} 4.4$ & $105.1290 \pm 0.0444$ & $13.209 \pm 0.021$ & $0.223 \pm 0.024$ & +0.20 & 7 & \\
\hline GJ 1128 & 12.78 & 1.774 & 3144 & 3112 & $3128 \pm 16$ & $\mathrm{dM} 4.6$ & $153.7816 \pm 0.0554$ & $13.715 \pm 0.021$ & $0.190 \pm 0.014$ & +0.09 & 6 & \\
\hline GJ $1151 \mathrm{~A}$ & 14.008 & 1.737 & 3169 & 3117 & $3143 \pm 26$ & $\mathrm{dM} 4.5$ & $124.4074 \pm 0.1186$ & $14.482 \pm 0.022$ & $0.129 \pm 0.010$ & +0.04 & 3 & \\
\hline GJ $1151 \mathrm{~B}$ & 14.008 & 1.737 & 3169 & 3117 & $3143 \pm 26$ & $\mathrm{dM} 4.5$ & $124.4074 \pm 0.1186$ & $14.482 \pm 0.022$ & $0.129 \pm 0.010$ & +0.04 & 3 & \\
\hline GJ 1154 & 13.64 & 1.780 & 3140 & 3056 & $3098 \pm 42$ & $\mathrm{dM} 4.7 \mathrm{e}$ & $123.7746 \pm 0.1075$ & $14.103 \pm 0.022$ & $0.169 \pm 0.023$ & +0.25 & 11 & \\
\hline GJ 1156 & 13.793 & 1.945 & 3000 & 2986 & $2993 \pm 7$ & dM5.3e & $154.5077 \pm 0.1108$ & $14.738 \pm 0.022$ & $0.158 \pm 0.008$ & +0.14 & 7 & \\
\hline GJ 1171 & 14.74 & 1.813 & 3115 & - & $3115 \pm 32$ & $\mathrm{dM} 4.7$ & $66.7531 \pm 0.0813$ & $13.862 \pm 0.023$ & $0.182 \pm 0.020$ & +0.03 & - & \\
\hline GJ 1182 & 14.30 & 1.791 & 3133 & - & $3133 \pm 32$ & $\mathrm{dM} 4.6$ & $71.1140 \pm 0.3884$ & $13.560 \pm 0.032$ & $0.202 \pm 0.023$ & -0.27 & - & \\
\hline GJ $1183 \mathrm{~A}$ & 13.98 & 1.718 & 3181 & - & $3181 \pm 32$ & $\mathrm{dM} 4.4$ & $57.0747 \pm 0.0646$ & $12.762 \pm 0.022$ & $0.263 \pm 0.028$ & -0.07 & - & \\
\hline GJ 1186 & 15.30 & 1.734 & 3171 & 3124 & $3147 \pm 23$ & $\mathrm{dM} 4.5$ & $51.2402 \pm 0.0693$ & $13.848 \pm 0.023$ & $0.172 \pm 0.015$ & +0.20 & - & \\
\hline GJ 1210 & 14.02 & 1.780 & 3140 & - & $3140 \pm 32$ & $\mathrm{dM} 4.5$ & 78. \pm 5.3 & $13.480 \pm 0.163$ & $0.206 \pm 0.038$ & -0.02 & - & \\
\hline GJ 1214 & 14.665 & 1.765 & 3150 & 3060 & $3105 \pm 45$ & $\mathrm{dM} 4.7$ & $68.2653 \pm 0.1723$ & $13.836 \pm 0.025$ & $0.189 \pm 0.027$ & +0.19 & 93 & \\
\hline GJ 1215 & 15.12 & 1.873 & 3063 & - & $3063 \pm 32$ & dM4.9 & $80.7994 \pm 0.1696$ & $14.657 \pm 0.025$ & $0.141 \pm 0.016$ & +0.07 & - & \\
\hline GJ 1223 & 14.89 & 1.826 & 3104 & - & $3104 \pm 32$ & $\mathrm{dM} 4.7$ & $81.8797 \pm 0.0588$ & $14.456 \pm 0.022$ & $0.142 \pm 0.016$ & -0.09 & - & \\
\hline GJ 1224 & 13.56 & 1.828 & 3102 & 3146 & $3124 \pm 22$ & $\mathrm{dM} 4.6 \mathrm{e}$ & $125.5872 \pm 0.0705$ & $14.055 \pm 0.021$ & $0.164 \pm 0.014$ & +0.01 & 16 & \\
\hline GJ 1225 & 15.385 & 1.697 & 3195 & - & $3195 \pm 32$ & $\mathrm{dM} 4.3$ & $52.9885 \pm 0.0441$ & $14.006 \pm 0.022$ & $0.144 \pm 0.015$ & -0.49 & - & Sudwarf? \\
\hline GJ 1227 & 13.415 & 1.745 & 3163 & 3161 & $3162 \pm 1$ & $\mathrm{dM} 4.4$ & 120. \pm 2.2 & $13.811 \pm 0.059$ & $0.169 \pm 0.009$ & +0.16 & 13 & \\
\hline GJ $1230 \mathrm{~A}$ & 12.35 & 1.749 & 3161 & 3152 & $3156 \pm 4$ & $\mathrm{dM} 4.5$ & $100.7234 \pm 0.0493$ & $12.366 \pm 0.021$ & $0.333 \pm 0.014$ & +0.26 & 2 & Binary? \\
\hline GJ $1230 \mathrm{~B}$ & 14.40 & 1.749 & 3161 & - & $3161 \pm 32$ & $\mathrm{sdM} 4.5 \mathrm{e}$ & $100.6830 \pm 0.0708$ & $14.415 \pm 0.022$ & $0.128 \pm 0.014$ & +0.34 & 2 & \\
\hline GJ 1231 & 15.19 & 1.780 & 3140 & 3161 & $3150 \pm 11$ & $\mathrm{dM} 4.5$ & $52.4454 \pm 0.0515$ & $13.789 \pm 0.022$ & $0.175 \pm 0.010$ & +0.28 & - & \\
\hline GJ $1232 \mathrm{~A}$ & 14.30 & 1.707 & 3188 & 3042 & $3115 \pm 73$ & $\mathrm{dM} 4.7$ & $97.4518 \pm 0.1596$ & $14.247 \pm 0.024$ & $0.153 \pm 0.029$ & +0.07 & 17 & \\
\hline GJ $1232 B$ & 14.30 & 1.707 & 3188 & 3042 & $3115 \pm 73$ & $\mathrm{dM} 4.7$ & $97.4518 \pm 0.1596$ & $14.247 \pm 0.024$ & $0.153 \pm 0.029$ & +0.07 & 17 & \\
\hline GJ 1253 & 14.167 & 1.837 & 3094 & 3023 & $3058 \pm 35$ & $\mathrm{dM} 4.9 \mathrm{e}$ & $104.3181 \pm 0.0524$ & $14.259 \pm 0.021$ & $0.172 \pm 0.020$ & & - & \\
\hline GJ 1256 & 13.41 & 1.712 & 3185 & 3089 & $3137 \pm 48$ & $\mathrm{dM} 4.6$ & $104.8892 \pm 0.0851$ & $13.514 \pm 0.022$ & $0.204 \pm 0.030$ & +0.22 & 12 & \\
\hline GJ 1268 & 14.935 & 1.735 & 3170 & 3014 & $3092 \pm 78$ & $\mathrm{dM} 4.8$ & $63.7940 \pm 0.0624$ & $13.959 \pm 0.022$ & $0.183 \pm 0.042$ & +0.07 & - & \\
\hline GJ 1277 & 14.010 & 1.780 & 3140 & 3095 & $3117 \pm 23$ & $\mathrm{dM} 4.7$ & $96.2224 \pm 0.0616$ & $13.926 \pm 0.021$ & $0.176 \pm 0.016$ & & - & \\
\hline GJ 1286 & 14.695 & 1.924 & 3019 & - & $3019 \pm 32$ & $\mathrm{dM} 5.2$ & $139.3100 \pm 0.1066$ & $15.415 \pm 0.022$ & $0.110 \pm 0.012$ & +0.06 & - & Subdwarf? \\
\hline GJ 1288 & 14.355 & 1.765 & 3150 & 3107 & $3128 \pm 21$ & $\mathrm{dM} 4.6$ & $77.9876 \pm 0.1206$ & $13.815 \pm 0.023$ & $0.181 \pm 0.015$ & -0.26 & - & \\
\hline GJ 2021 & 14.54 & 1.729 & 3174 & - & $3174 \pm 32$ & $\mathrm{dM} 4.4$ & $55.9197 \pm 0.0704$ & $13.278 \pm 0.023$ & $0.211 \pm 0.023$ & & - & \\
\hline GJ $2069 \mathrm{~B}$ & 14.83 & 1.712 & 3185 & 3131 & $3158 \pm 27$ & $\mathrm{dM} 4.5 \mathrm{e}$ & $62.0284 \pm 0.2144$ & $13.793 \pm 0.027$ & $0.172 \pm 0.017$ & +0.23 & 3 & \\
\hline GJ 3033 & 14.545 & 1.758 & 3155 & 3184 & $3169 \pm 15$ & $\mathrm{dM} 4.4$ & $51.8335 \pm 0.1448$ & $13.118 \pm 0.026$ & $0.229 \pm 0.016$ & +0.20 & - & \\
\hline GJ 3052 & 13.8 & 1.701 & 3192 & - & $3192 \pm 32$ & $\mathrm{dM} 4.3$ & $37.5717 \pm 0.3450$ & $11.674 \pm 0.040$ & $0.424 \pm 0.049$ & +0.09 & - & Binary? \\
\hline GJ 3053 & 14.19 & 1.719 & 3181 & - & $3181 \pm 32$ & $\mathrm{dM} 4.4$ & $66.6996 \pm 0.0674$ & $13.311 \pm 0.022$ & $0.204 \pm 0.022$ & & - & \\
\hline GJ 3119 & 14.49 & 1.885 & 3053 & 3050 & $3051 \pm 1$ & dM5.0 & $94.4945 \pm 0.0968$ & $14.367 \pm 0.022$ & $0.166 \pm 0.007$ & -0.03 & - & \\
\hline GJ 3247 & 14.59 & 1.764 & 3151 & 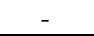 & $3151 \pm 32$ & $\mathrm{dM} 4.5$ & $49.6467 \pm 0.2539$ & $13.069 \pm 0.031$ & $0.243 \pm 0.028$ & & - & \\
\hline
\end{tabular}


Table 2:: continued.

\begin{tabular}{|c|c|c|c|c|c|c|c|c|c|c|c|c|}
\hline Star & $\begin{array}{c}\mathrm{V} \\
(\mathrm{mag})\end{array}$ & $\begin{array}{c}(\mathrm{R}-\mathrm{I})_{C} \\
(\mathrm{mag})\end{array}$ & $\begin{array}{c}T_{\text {eff }}{ }^{1} \\
(\mathrm{~K})\end{array}$ & $\begin{array}{c}T_{e f f}{ }^{2} \\
(\mathrm{~K})\end{array}$ & $\begin{array}{c}T_{\text {eff }} \pm 3 \sigma^{3} \\
(\mathrm{~K})\end{array}$ & $\begin{array}{l}\text { Spect. } \\
\text { Type }\end{array}$ & $\begin{array}{c}\pi \\
(\mathrm{m} ")\end{array}$ & $\begin{array}{c}M_{V} \\
(\mathrm{mag})\end{array}$ & $\begin{array}{c}R_{\star} \\
\left(R_{\odot}\right)\end{array}$ & {$[\mathrm{M} / \mathrm{H}]$} & $\begin{array}{l}\text { Hi. Res. } \\
\text { Spectra }\end{array}$ & Comments \\
\hline GJ 3250 & 14.51 & 1.746 & 3163 & _- & $3163 \pm 32$ & $\mathrm{dM} 4.4$ & $80.6267 \pm 0.1040$ & $14.042 \pm 0.023$ & $0.152 \pm 0.016$ & -0.03 & - & \\
\hline GJ 3253 & 13.79 & 1.778 & 3142 & 3061 & $3101 \pm 40$ & $\mathrm{dM} 4.7(\mathrm{e})$ & $103.3802 \pm 0.1287$ & $13.862 \pm 0.023$ & $0.188 \pm 0.025$ & +0.02 & 13 & \\
\hline GJ 3296 & 14.60 & 1.749 & 3161 & 3064 & $3112 \pm 48$ & $\mathrm{dM} 4.7$ & $73.5425 \pm 0.0975$ & $13.933 \pm 0.023$ & $0.178 \pm 0.027$ & +0.11 & - & \\
\hline GJ 3323 & 12.166 & 1.706 & 3189 & 3158 & $3173 \pm 15$ & $\mathrm{dM} 4.4 \mathrm{e}$ & $186.0231 \pm 0.0590$ & $13.514 \pm 0.021$ & $0.189 \pm 0.013$ & & - & \\
\hline GJ 3380 & 14.541 & 1.902 & 3038 & 3022 & $3030 \pm 8$ & $\mathrm{dM} 5.1 \mathrm{e}$ & $104.8903 \pm 0.0746$ & $14.645 \pm 0.022$ & $0.152 \pm 0.009$ & & - & \\
\hline GJ 3421 & 13.32 & 1.786 & 3136 & 3031 & $3083 \pm 52$ & sdM4.8 & $109.6921 \pm 0.4015$ & $13.521 \pm 0.028$ & $0.228 \pm 0.037$ & -0.53 & 2 & \\
\hline GJ 3463 & 14.75 & 1.833 & 3098 & - & $3098 \pm 32$ & $\mathrm{dM} 4.7$ & $67.5288 \pm 0.0719$ & $13.897 \pm 0.022$ & $0.186 \pm 0.021$ & +0.62 & - & \\
\hline GJ 3469B & 15.509 & 1.712 & 3185 & - & $3185 \pm 32$ & $\mathrm{dM} 4.3$ & $32.0587 \pm 0.0935$ & $13.039 \pm 0.026$ & $0.230 \pm 0.025$ & & - & \\
\hline GJ 3518 & 14.0 & 1.734 & 3171 & - & $3171 \pm 32$ & $\mathrm{dM} 4.4$ & $69.2108 \pm 0.4575$ & $13.201 \pm 0.034$ & $0.220 \pm 0.025$ & & - & \\
\hline GJ 3533 & 14.21 & 1.781 & 3140 & - & $3140 \pm 32$ & $\mathrm{dM} 4.5$ & $65.2087 \pm 0.1163$ & $13.282 \pm 0.024$ & $0.226 \pm 0.025$ & & - & \\
\hline GJ 3542 & 15.12 & 1.777 & 3142 & 3028 & $3085 \pm 57$ & $\mathrm{dM} 4.8$ & $64.1656 \pm 0.0575$ & $14.157 \pm 0.022$ & $0.170 \pm 0.029$ & & - & \\
\hline GJ 3571 & 14.05 & 1.802 & 3125 & 3089 & $3107 \pm 18$ & $\mathrm{dM} 4.7 \mathrm{e}$ & $104.5013 \pm 0.1153$ & $14.146 \pm 0.022$ & $0.163 \pm 0.013$ & & 4 & \\
\hline GJ 3618 & 13.920 & 1.888 & 3050 & 3000 & $3025 \pm 25$ & dM5.1 & $206.8172 \pm 0.0735$ & $15.498 \pm 0.021$ & $0.104 \pm 0.010$ & +0.38 & 7 & \\
\hline GJ 3631 & 14.803 & 1.873 & 3063 & 3039 & $3051 \pm 12$ & $\mathrm{dM} 4.9 \mathrm{e}$ & $71.7830 \pm 0.0903$ & $14.083 \pm 0.023$ & $0.189 \pm 0.012$ & & - & \\
\hline GJ 3668 & 14.170 & 1.781 & 3140 & 3037 & $3088 \pm 51$ & dM5.0 & $87.0730 \pm 0.1018$ & $13.869 \pm 0.023$ & $0.193 \pm 0.030$ & -0.07 & - & \\
\hline GJ 3737 & 12.74 & 1.735 & 3170 & 3074 & $3122 \pm 48$ & $\mathrm{dM} 4.6$ & $149.8423 \pm 0.0755$ & $13.618 \pm 0.021$ & $0.201 \pm 0.030$ & -0.25 & 16 & \\
\hline GJ 3816 & 15.262 & 1.792 & 3133 & 8017 & $3133 \pm 32$ & dM4.6 & $\begin{array}{l}53 . \pm 8 \\
\text {. }\end{array}$ & $13.883 \pm 0.325$ & $0.174 \pm 0.048$ & & 10 & \\
\hline GJ 3820 & 12.88 & 1.730 & 3173 & 3152 & $3162 \pm 10$ & $\mathrm{dM} 4.5 \mathrm{e}$ & $92.4336 \pm 0.0769$ & $12.709 \pm 0.022$ & $0.281 \pm 0.016$ & +0.11 & 8 & Binary? \\
\hline GJ 3853 & 14.66 & 1.702 & 3192 & - & $3192 \pm 32$ & $\mathrm{dM} 4.3$ & $38.2352 \pm 0.0487$ & $12.572 \pm 0.023$ & $0.281 \pm 0.030$ & & - & Binary? \\
\hline GJ 3878A & 15.74 & 1.797 & 3129 & 2999 & $3064 \pm 65$ & dM4.9 & $53.4150 \pm 0.0721$ & $14.378 \pm 0.023$ & $0.160 \pm 0.031$ & -0.03 & - & \\
\hline GJ 3910 & 14.30 & 1.708 & 3188 & 3215 & $3201 \pm 14$ & dM4.3 & $34.7132 \pm 0.3842$ & $12.002 \pm 0.044$ & $0.358 \pm 0.026$ & -0.23 & - & Binary? \\
\hline GJ 3937 & 15.44 & 1.819 & 3110 & - & $3110 \pm 32$ & $\mathrm{dM} 4.7$ & $56.9325 \pm 0.1111$ & $14.217 \pm 0.024$ & $0.157 \pm 0.017$ & & - & \\
\hline GJ 3954 & 14.31 & 1.788 & 3135 & - & $3135 \pm 32$ & dM4.6 & $\begin{array}{l}\text { 84. } \pm 13 \\
\text {. }\end{array}$ & $13.931 \pm 0.332$ & $0.169 \pm 0.047$ & & - & \\
\hline GJ 3976 & 14.027 & 1.835 & 3096 & 3054 & $3075 \pm 21$ & $\mathrm{dM} 4.8 \mathrm{e}$ & $95.5605 \pm 0.0619$ & $13.928 \pm 0.021$ & $0.193 \pm 0.016$ & & - & \\
\hline GJ 3988 & 13.57 & 1.721 & 3179 & 3093 & $3136 \pm 43$ & $\mathrm{dM} 4.6$ & $100.8741 \pm 0.0420$ & $13.589 \pm 0.021$ & $0.198 \pm 0.027$ & -0.33 & - & \\
\hline GJ 4053 & 13.475 & 1.726 & 3176 & 3109 & $3142 \pm 33$ & $\mathrm{dM} 4.5$ & $130.7722 \pm 0.0443$ & $14.058 \pm 0.021$ & $0.157 \pm 0.017$ & -0.08 & - & \\
\hline GJ 4215B & 14.81 & 1.735 & 3170 & - & $3170 \pm 32$ & $\mathrm{dM} 4.4$ & $58.5163 \pm 0.0741$ & $13.646 \pm 0.023$ & $0.179 \pm 0.019$ & +0.21 & - & \\
\hline GJ 4235 & 15.35 & 1.708 & 3188 & - & $3188 \pm 32$ & dM4.3 & $37.5630 \pm 0.1574$ & $13.224 \pm 0.029$ & $0.210 \pm 0.023$ & & - & \\
\hline GJ 4239 & 14.64 & 1.780 & 3140 & - & $3140 \pm 32$ & dM4.5 & $74.1413 \pm 0.0980$ & $13.990 \pm 0.023$ & $0.163 \pm 0.018$ & & - & \\
\hline GJ 4243B & 15.8 & 1.865 & 3070 & - & $3070 \pm 32$ & $\mathrm{dM} 4.9$ & $51.3373 \pm 0.5946$ & $14.352 \pm 0.225$ & $0.160 \pm 0.035$ & & - & \\
\hline GJ 4274 & 13.275 & 1.785 & 3137 & 3097 & $3117 \pm 20$ & $\mathrm{dM} 4.7 \mathrm{e}$ & $138.1782 \pm 0.2484$ & $13.977 \pm 0.024$ & $0.172 \pm 0.014$ & +0.29 & 9 & \\
\hline GJ 4335 & 15.75 & 1.777 & 3142 & - & $3142 \pm 32$ & $\mathrm{dM} 4.5$ & $44.3829 \pm 0.0911$ & $13.986 \pm 0.024$ & $0.163 \pm 0.018$ & & - & \\
\hline GJ 4360 & 14.5 & 1.923 & 3020 & - & $3020 \pm 32$ & dM5.1 & 111. \pm 19 & $14.727 \pm 0.543$ & $0.150 \pm 0.062$ & & 2 & \\
\hline GJ 4371 & 15.5 & 1.816 & 3113 & - & $3113 \pm 32$ & dM4.7 & $42.3030 \pm 0.5546$ & $13.632 \pm 0.228$ & $0.204 \pm 0.045$ & & - & \\
\hline Gl 31.2B & 15.90 & 1.712 & 3185 & 3080 & $3132 \pm 52$ & dM4.6 & $27.4741 \pm 0.5302$ & $13.095 \pm 0.062$ & $0.251 \pm 0.045$ & +0.32 & - & \\
\hline Gl $49 \mathrm{~B}$ & 13.78 & 1.919 & 3023 & - & $3023 \pm 32$ & dM5.1 & $101.4650 \pm 0.0335$ & $13.812 \pm 0.021$ & $0.227 \pm 0.026$ & +0.08 & - & \\
\hline Gl 54.1 & 12.074 & 1.782 & 3139 & 3063 & $3101 \pm 38$ & $\mathrm{dM} 4.7(\mathrm{e})$ & $269.3628 \pm 0.0785$ & $14.226 \pm 0.021$ & $0.159 \pm 0.020$ & -0.35 & 126 & \\
\hline Gl 83.1 & 12.297 & 1.753 & 3158 & 3112 & $3135 \pm 23$ & $\mathrm{dM} 4.6 \mathrm{e}$ & $223.6349 \pm 0.1066$ & $14.045 \pm 0.021$ & $0.161 \pm 0.014$ & -0.14 & 29 & \\
\hline Gl $166 \mathrm{C}$ & 11.230 & 1.726 & 3176 & 3167 & $\begin{array}{l}3171 \pm 4 \\
3171\end{array}$ & $\mathrm{dM} 4.4 \mathrm{e}$ & $199.4552 \pm 0.3204$ & $12.729 \pm 0.023$ & $0.273 \pm 0.012$ & & - & Binary? \\
\hline Gl 170 & 14.10 & 1.84 & 3092 & - & $3092 \pm 32$ & $\mathrm{dM} 4.8 \mathrm{e}$ & $91.7968 \pm 0.0771$ & $13.914 \pm 0.022$ & $0.187 \pm 0.021$ & -0.05 & - & \\
\hline Gl $234 \mathrm{~A}$ & 11.824 & 1.760 & 3154 & 3104 & $3129 \pm 25$ & $\mathrm{dM} 4.6 \mathrm{e}$ & $242.9659 \pm 0.8833$ & $13.752 \pm 0.028$ & $0.186 \pm 0.018$ & +0.11 & 20 & \\
\hline Gl $234 \mathrm{~B}$ & 11.824 & 1.760 & 3154 & 3104 & $3129 \pm 25$ & $\mathrm{dM} 4.6$ & $242.9659 \pm 0.8833$ & $13.752 \pm 0.028$ & $0.186 \pm 0.018$ & +0.11 & 20 & \\
\hline Gl $268 \mathrm{~A}$ & 12.258 & 1.735 & 3170 & 3118 & $3144 \pm 26$ & $\mathrm{dM} 4.5 \mathrm{e}$ & $164.6395 \pm 0.1338$ & $13.341 \pm 0.022$ & $0.218 \pm 0.021$ & +0.14 & 2 & \\
\hline Gl 268B & 12.258 & 1.735 & 3170 & 3118 & $3144 \pm 26$ & $\mathrm{dM} 4.5 \mathrm{e}$ & $164.6395 \pm 0.1338$ & $13.341 \pm 0.022$ & $0.218 \pm 0.021$ & +0.14 & 2 & \\
\hline Gl 285 & 11.225 & 1.745 & 3164 & 3173 & $3168 \pm 5$ & $\mathrm{dM} 4.4 \mathrm{e}$ & $167.0186 \pm 0.0592$ & $12.339 \pm 0.021$ & $0.329 \pm 0.015$ & +0.87 & 21 & \\
\hline Gl 299 & 13.030 & 1.746 & 3163 & 3112 & $3137 \pm 25$ & dM4.6 & $145.4757 \pm 0.5695$ & $13.844 \pm 0.028$ & $0.176 \pm 0.017$ & -0.43 & 27 & \\
\hline Gl 347B & 15.00 & 1.723 & 3178 & 3080 & $3129 \pm 49$ & dM4.6 & $57.1832 \pm 0.0668$ & $13.786 \pm 0.023$ & $0.183 \pm 0.028$ & -0.13 & 2 & \\
\hline Gl 359 & 14.22 & 1.73 & 3173 & 3018 & $3095 \pm 77$ & $\mathrm{dM} 4.8$ & $81.2607 \pm 0.0976$ & $13.769 \pm 0.023$ & $0.199 \pm 0.045$ & -0.33 & - & \\
\hline Gl 467B & 15.75 & 1.700 & 3193 & - & $3193 \pm 32$ & sdM4.3 & $46.8103 \pm 0.0443$ & $14.102 \pm 0.022$ & $0.138 \pm 0.015$ & & - & Sudwarf? \\
\hline Gl 493.1 & 13.37 & 1.781 & 3140 & 3113 & $3126 \pm 13$ & $\mathrm{dM} 4.6 \mathrm{e}$ & $116.8096 \pm 0.1974$ & $13.707 \pm 0.024$ & $0.191 \pm 0.013$ & +0.20 & 11 & \\
\hline Gl 514.1 & 14.18 & 1.72 & 3180 & - & $3180 \pm 32$ & $\mathrm{dM} 4.4$ & $62.1427 \pm 0.0808$ & $13.147 \pm 0.023$ & $0.221 \pm 0.024$ & -0.24 & - & \\
\hline Gl 540.2 & 13.875 & 1.73 & 3173 & 3540 & $3357 \pm 183$ & $\mathrm{sdM} 3.5$ & $31.7829 \pm 0.0813$ & $11.386 \pm 0.026$ & $0.353 \pm 0.162$ & -0.80 & - & Binary? \\
\hline Gl $541.2 \mathrm{~B}$ & 14.155 & 1.701 & 3192 & - & $3192 \pm 32$ & sdM4.3 & $51.7439 \pm 0.1611$ & $12.724 \pm 0.027$ & $0.262 \pm 0.029$ & -0.92 & 13 & Binary? \\
\hline
\end{tabular}


Table 2:: continued.

\begin{tabular}{|c|c|c|c|c|c|c|c|c|c|c|c|c|}
\hline Star & $\begin{array}{c}\mathrm{V} \\
(\mathrm{mag})\end{array}$ & $\begin{array}{c}(\mathrm{R}-\mathrm{I})_{C} \\
(\mathrm{mag})\end{array}$ & $\begin{array}{c}T_{e f f}{ }^{1} \\
(\mathrm{~K})\end{array}$ & $\begin{array}{c}T_{e f f}^{2} \\
(\mathrm{~K})\end{array}$ & $\begin{array}{c}T_{\text {eff }} \pm 3 \sigma^{3} \\
(\mathrm{~K})\end{array}$ & $\begin{array}{l}\text { Spect. } \\
\text { Type }\end{array}$ & $\begin{array}{c}\pi \\
(\mathrm{m} ")\end{array}$ & $\begin{array}{c}M_{V} \\
(\mathrm{mag})\end{array}$ & $\begin{array}{c}R_{\star} \\
\left(R_{\odot}\right)\end{array}$ & {$[\mathrm{M} / \mathrm{H}]$} & $\begin{array}{l}\text { Hi. Res. } \\
\text { Spectra }\end{array}$ & Comments \\
\hline Gl 544B & 14.5 & 1.718 & 3181 & 3125 & $3153 \pm 28$ & $\mathrm{dM} 4.5$ & $48.6039 \pm 0.0951$ & $12.933 \pm 0.024$ & $0.258 \pm 0.026$ & -0.13 & 5 & \\
\hline Gl 585 & 13.720 & 1.721 & 3179 & 3164 & $3171 \pm 8$ & $\mathrm{dM} 4.4$ & $89.1750 \pm 0.0844$ & $13.471 \pm 0.022$ & $0.194 \pm 0.010$ & -0.22 & 3 & \\
\hline Gl 586C & 15.42 & 1.780 & 3140 & 3070 & $3105 \pm 35$ & dM4.7 & $47.5732 \pm 0.0942$ & $13.807 \pm 0.024$ & $0.191 \pm 0.023$ & & - & \\
\hline Gl 589B & 14.94 & 1.749 & 3161 & - & $3161 \pm 32$ & $\mathrm{dM} 4.5$ & $66.8409 \pm 0.0716$ & $14.065 \pm 0.022$ & $0.151 \pm 0.016$ & -0.25 & - & \\
\hline Gl 669B & 12.99 & 1.75 & 3160 & 3161 & $3160 \pm 1$ & $\mathrm{dM} 4.5$ & $92.9885 \pm 0.0498$ & $12.832 \pm 0.021$ & $0.266 \pm 0.010$ & +0.03 & 3 & \\
\hline Gl $695 \mathrm{C}$ & 12.66 & - & - & 3100 & $3100 \pm 32$ & $\mathrm{dM} 4.7$ & $119.7908 \pm 0.6586$ & $13.052 \pm 0.032$ & $0.273 \pm 0.032$ & +0.39 & - & \\
\hline Gl $766 \mathrm{~A}$ & 13.08 & 1.71 & 3187 & 3142 & $3164 \pm 22$ & $\mathrm{dM} 4.4$ & $94.0139 \pm 0.0804$ & $12.946 \pm 0.022$ & $0.251 \pm 0.021$ & +0.04 & 2 & \\
\hline Gl 766B & 13.08 & 1.71 & 3187 & 3142 & $3164 \pm 22$ & $\mathrm{dM} 4.4$ & $93.9479 \pm 0.0756$ & $12.944 \pm 0.022$ & $0.251 \pm 0.021$ & +0.04 & 2 & \\
\hline Gl $773 \mathrm{~B}$ & 15.36 & 1.909 & 3032 & - & $3032 \pm 32$ & dM5.1 & $56.2226 \pm 0.0974$ & $14.110 \pm 0.024$ & $0.194 \pm 0.022$ & -0.10 & - & \\
\hline Gl $777 \mathrm{~B}$ & 14.365 & 1.768 & 3148 & 3083 & $3115 \pm 32$ & $\mathrm{dM} 4.6$ & $62.5879 \pm 0.0577$ & $13.347 \pm 0.022$ & $0.231 \pm 0.025$ & +0.10 & - & \\
\hline Gl $791.2 \mathrm{~A}$ & 13.05 & 1.709 & 3187 & 3115 & $3151 \pm 36$ & $\mathrm{dM} 4.5 \mathrm{e}$ & $133.8113 \pm 1.3869$ & $13.682 \pm 0.042$ & $0.184 \pm 0.024$ & +0.05 & 123 & \\
\hline Gl $799 \mathrm{~A}$ & 10.343 & 1.697 & 3195 & - & $3195 \pm 32$ & $\mathrm{dM} 4.3 \mathrm{e}$ & $101.1982 \pm 0.0792$ & $10.369 \pm 0.022$ & $0.769 \pm 0.082$ & & 58 & PMS? \\
\hline Gl 799B & 11.34 & 1.697 & 3195 & - & $3195 \pm 32$ & $\mathrm{dM} 4.3 \mathrm{e}$ & $101.7170 \pm 0.0956$ & $11.377 \pm 0.022$ & $0.484 \pm 0.052$ & & 58 & PMS \\
\hline Gl $802 \mathrm{~A}$ & 15.44 & 1.856 & 3078 & 2939 & $3008 \pm 70$ & $\mathrm{dM} 5.2 \mathrm{e}$ & $58.3160 \pm 0.1412$ & $14.272 \pm 0.025$ & $0.190 \pm 0.035$ & -0.25 & 19 & \\
\hline Gl $802 \mathrm{~B}$ & 15.44 & 1.856 & 3078 & 2939 & $3008 \pm 70$ & $\mathrm{dM} 5.2 \mathrm{e}$ & $58.3160 \pm 0.1412$ & $14.272 \pm 0.025$ & $0.190 \pm 0.035$ & -0.25 & 19 & \\
\hline Gl $810 B$ & 14.61 & 1.787 & 3136 & - & $3136 \pm 32$ & $\mathrm{dM} 4.6$ & $80.2319 \pm 0.0881$ & $14.132 \pm 0.022$ & $0.154 \pm 0.017$ & +0.02 & - & \\
\hline Gl 831 & 12.006 & 1.698 & 3194 & 3095 & $3145 \pm 50$ & $\mathrm{dM} 4.5(\mathrm{e})$ & $134.0831 \pm 1.1125$ & $12.643 \pm 0.038$ & $0.300 \pm 0.048$ & +0.30 & 6 & Binary? \\
\hline Gl $852 \mathrm{~B}$ & 14.47 & 1.827 & 3103 & - & $3103 \pm 32$ & $\mathrm{dM} 4.7$ & $89.2041 \pm 0.1283$ & $14.222 \pm 0.023$ & $0.159 \pm 0.018$ & & - & \\
\hline Gl 896B & 12.096 & 1.747 & 3154 & 3149 & $3152 \pm 2$ & $\mathrm{dM} 4.5 \mathrm{e}$ & $160.0598 \pm 0.1079$ & $13.117 \pm 0.021$ & $0.238 \pm 0.013$ & +0.10 & - & \\
\hline Gl $905 \mathrm{~A}$ & 13.048 & 1.909 & 3032 & 3047 & $3039 \pm 8$ & $\mathrm{dM} 5.0(\mathrm{e})$ & $316.9558 \pm 0.1260$ & $15.553 \pm 0.021$ & $0.098 \pm 0.003$ & +0.21 & 5 & Sudwarf? \\
\hline Gl $905 \mathrm{~B}$ & 13.048 & 1.909 & 3032 & 3047 & $3039 \pm 8$ & dM5.0(e) & $316.9558 \pm 0.1260$ & $15.553 \pm 0.021$ & $0.098 \pm 0.003$ & +0.21 & 5 & Sudwarf? \\
\hline LHS 269 & 16.443 & 1.947 & 2999 & - & $2999 \pm 32$ & dM5.3 & $56.7136 \pm 0.0924$ & $15.211 \pm 0.024$ & $0.126 \pm 0.015$ & & - & \\
\hline LHS 279 & 16.461 & 1.824 & 3106 & - & $3106 \pm 32$ & $\mathrm{dM} 4.7$ & $24.3253 \pm 0.0574$ & $13.391 \pm 0.025$ & $0.231 \pm 0.026$ & & - & \\
\hline LHS 370 & 16.17 & 1.82 & 3109 & - & $3109 \pm 32$ & $\mathrm{dM} 4.7$ & $46.2825 \pm 0.0602$ & $14.497 \pm 0.023$ & $0.138 \pm 0.015$ & -0.42 & - & \\
\hline LHS 1240 & 13.7 & 1.727 & 3175 & - & $3175 \pm 32$ & $\mathrm{dM} 4.4$ & $65.5108 \pm 0.3115$ & $12.782 \pm 0.210$ & $0.264 \pm 0.055$ & +0.21 & - & \\
\hline LHS 1460 & 14.7 & 1.841 & 3091 & - & $3091 \pm 32$ & $\mathrm{dM} 4.8$ & $21.4784 \pm 0.0648$ & $11.360 \pm 0.207$ & $0.608 \pm 0.127$ & & - & PMS? \\
\hline LHS 2204 & 16.83 & 1.792 & 3133 & - & $3133 \pm 32$ & $\operatorname{sdM} 4.6$ & $29.2823 \pm 0.1226$ & $14.163 \pm 0.029$ & $0.153 \pm 0.017$ & -0.51 & - & \\
\hline LHS 2602 & 16.0 & 1.896 & 3043 & - & $3043 \pm 32$ & dM5.0 & $29.9435 \pm 0.0633$ & $13.382 \pm 0.205$ & $0.265 \pm 0.056$ & & - & \\
\hline LHS 2619 & 15.7 & 1.716 & 3183 & - & $3183 \pm 32$ & $\mathrm{dM} 4.4$ & $46.2067 \pm 0.0824$ & $14.024 \pm 0.204$ & $0.147 \pm 0.030$ & & - & \\
\hline LHS 2643 & 17.43 & 1.85 & 3083 & - & $3083 \pm 32$ & $\mathrm{dM} 4.8$ & $27.2465 \pm 0.0968$ & $14.607 \pm 0.028$ & $0.139 \pm 0.016$ & +0.31 & - & \\
\hline LHS 3196 & 16.32 & 1.736 & 3169 & - & $3169 \pm 32$ & $\mathrm{dM} 4.4$ & $36.1586 \pm 0.0509$ & $14.111 \pm 0.023$ & $0.145 \pm 0.016$ & -0.30 & - & \\
\hline LSPM J0336+3118 & 13.995 & 1.787 & 3136 & 3109 & $3122 \pm 13$ & dM4.6 & $73.0658 \pm 0.1258$ & $13.314 \pm 0.024$ & $0.213 \pm 0.015$ & & - & \\
\hline & & & & & dM6 sample & & & & & & & \\
\hline GJ 1002 & 13.765 & 1.980 & 2970 & - & $2970 \pm 18$ & dM5.5 & $206.2134 \pm 0.1281$ & $15.337 \pm 0.021$ & $0.127 \pm 0.011$ & -0.15 & 12 & \\
\hline GJ 1061 & 13.06 & 1.970 & 2979 & 2999 & $2989 \pm 10$ & dM5.4 & $272.2446 \pm 0.0661$ & $15.235 \pm 0.021$ & $0.127 \pm 0.008$ & -0.08 & 42 & \\
\hline GJ $1083 \mathrm{~A}$ & 15.646 & 2.08 & 2905 & - & $2905 \pm 18$ & dM5.9 & $95 . \pm 3$ & $15.535 \pm 0.088$ & $0.135 \pm 0.016$ & +0.12 & - & \\
\hline GJ 1083B & 15.646 & 2.08 & 2905 & - & $2905 \pm 18$ & dM5.9 & $95 . \pm 3$ & $15.535 \pm 0.088$ & $0.135 \pm 0.016$ & +0.12 & - & \\
\hline GJ 1093 & 14.755 & 1.945 & 3000 & - & $3000 \pm 18$ & dM5.3 & $129.0023 \pm 0.1681$ & $15.308 \pm 0.023$ & $0.120 \pm 0.010$ & & 1 & \\
\hline GJ 1116A & 14.503 & 2.123 & 2887 & 2912 & $2899 \pm 13$ & $\mathrm{dM} 6.0$ & $194.7225 \pm 0.1251$ & $15.950 \pm 0.021$ & $0.113 \pm 0.009$ & -0.29 & 5 & \\
\hline GJ 1116B & 14.503 & 2.123 & 2887 & 2912 & $2899 \pm 13$ & dM6.0 & $195.0836 \pm 0.1754$ & $15.954 \pm 0.022$ & $0.113 \pm 0.009$ & -0.29 & 5 & \\
\hline GJ 1238 & 15.365 & 1.967 & 2981 & - & $2981 \pm 18$ & dM5.4 & $94.0738 \pm 0.0551$ & $15.232 \pm 0.021$ & $0.130 \pm 0.011$ & & - & \\
\hline GJ $1245 \mathrm{~A}$ & 13.41 & 2.01 & 2944 & 2964 & $2954 \pm 10$ & dM5.6 & $213.1329 \pm 0.5737$ & $15.053 \pm 0.026$ & $0.150 \pm 0.010$ & -0.06 & 5 & \\
\hline GJ 1245B & 13.957 & 2.095 & 2898 & 2910 & $2904 \pm 6$ & $\mathrm{dM} 5.9 \mathrm{e}$ & $214.5285 \pm 0.0824$ & $15.614 \pm 0.021$ & $0.130 \pm 0.007$ & -0.04 & 5 & \\
\hline GJ 1286 & 14.685 & 2.018 & 2937 & 2931 & $2934 \pm 3$ & dM5.7 & $139.3100 \pm 0.1066$ & $15.405 \pm 0.022$ & $0.134 \pm 0.007$ & +0.06 & - & \\
\hline GJ 2005 & 15.338 & 2.125 & 2887 & - & $2887 \pm 18$ & dM6.1 & $129.47 \pm 2.48$ & $15.899 \pm 0.061$ & $0.119 \pm 0.013$ & & 2 & \\
\hline GJ 3146 & 15.758 & 2.165 & 2868 & 2877 & $2872 \pm 5$ & dM6.3 & $109.8030 \pm 0.1932$ & $15.961 \pm 0.024$ & $0.120 \pm 0.007$ & & - & \\
\hline GJ 3147 & 15.850 & 2.102 & 2895 & 2858 & $2876 \pm 18$ & $\mathrm{dM} 6.2$ & $96.7195 \pm 0.1109$ & $15.778 \pm 0.022$ & $0.129 \pm 0.012$ & & - & \\
\hline Gl 51 & 13.66 & 1.937 & 3008 & 3065 & $3037 \pm 29$ & dM5.1 & $101.6371 \pm 0.0806$ & $13.695 \pm 0.022$ & $0.233 \pm 0.019$ & +0.26 & - & Binary? \\
\hline Gl 65A & 12.813 & 2.060 & 2913 & 3025 & $2969 \pm 56$ & dM5.5 & $369.9318 \pm 0.2684$ & $15.654 \pm 0.022$ & $0.110 \pm 0.018$ & -0.21 & 3 & \\
\hline Gl 412B & 14.360 & 2.127 & 2885 & 2870 & $2877 \pm 7$ & sdM6.2 & $204.0592 \pm 0.1687$ & $15.909 \pm 0.022$ & $0.121 \pm 0.007$ & -0.56 & - & \\
\hline Gl $473 \mathrm{~A}$ & 13.220 & 1.977 & 2973 & 2959 & $2966 \pm 7$ & dM5.5 & $\begin{array}{l}227 . \pm 5 \\
2 .\end{array}$ & $15.000 \pm 0.067$ & $0.150 \pm 0.012$ & +0.10 & - & \\
\hline
\end{tabular}


Table 2:: continued.

\begin{tabular}{|c|c|c|c|c|c|c|c|c|c|c|c|c|}
\hline Star & $\begin{array}{c}\mathrm{V} \\
(\mathrm{mag})\end{array}$ & $\begin{array}{c}(\mathrm{R}-\mathrm{I})_{C} \\
(\mathrm{mag})\end{array}$ & $\begin{array}{c}T_{\text {eff }}{ }^{1} \\
(\mathrm{~K})\end{array}$ & $\begin{array}{c}T_{e f f}{ }^{2} \\
(\mathrm{~K})\end{array}$ & $\begin{array}{c}T_{\text {eff }} \pm 3 \sigma^{3} \\
(\mathrm{~K})\end{array}$ & $\begin{array}{l}\text { Spect. } \\
\text { Type }\end{array}$ & $\begin{array}{c}\pi \\
(\mathrm{m} ")\end{array}$ & $\begin{array}{c}M_{V} \\
(\mathrm{mag})\end{array}$ & $\begin{array}{c}R_{\star} \\
\left(R_{\odot}\right)\end{array}$ & {$[\mathrm{M} / \mathrm{H}]$} & $\begin{array}{l}\text { Hi. Res. } \\
\text { Spectra }\end{array}$ & Comments \\
\hline Gl 473B & 13.220 & 1.977 & 2973 & 2959 & $2966 \pm 7$ & dM5.5 & 227. \pm 5 & $15.000 \pm 0.067$ & $0.150 \pm 0.012$ & +0.10 & - & \\
\hline Gl 551 & 11.11 & 2.03 & 2927 & 2953 & $2940 \pm 13$ & dM5.8 & $768.13 \pm 1.04$ & $15.537 \pm 0.023$ & $0.124 \pm 0.009$ & +0.08 & 91 & \\
\hline Gl 618B & 15.135 & 1.890 & 3048 & 2920 & $2984 \pm 64$ & dM5.4 & $117.3560 \pm 0.0598$ & $15.483 \pm 0.021$ & $0.115 \pm 0.020$ & -0.07 & - & \\
\hline Gl $866 \mathrm{~A}$ & 13.114 & 2.039 & 2922 & 2850 & $2886 \pm 36$ & $\mathrm{dM} 6.1$ & 300. \pm 5 & $15.500 \pm 0.056$ & $0.143 \pm 0.022$ & +0.14 & - & \\
\hline Gl 866B & 13.114 & 2.039 & 2922 & 2850 & $2886 \pm 36$ & $\mathrm{dM} 6.1$ & $300 . \pm 5$. & $15.500 \pm 0.056$ & $0.143 \pm 0.022$ & +0.14 & - & \\
\hline LHS 254 & 17.156 & 2.071 & 2909 & - & $2909 \pm 18$ & dM5.9 & $51.1989 \pm 0.0837$ & $15.702 \pm 0.024$ & $0.124 \pm 0.011$ & & - & \\
\hline LHS 2347 & 19.00 & 2.16 & 2871 & - & $2871 \pm 18$ & $\mathrm{dM} 6.3$ & $23.8575 \pm 0.1392$ & $15.888 \pm 0.033$ & $0.124 \pm 0.012$ & & - & \\
\hline LHS 2502 & 19.130 & 2.08 & 2905 & 2900 & $2902 \pm 2$ & $\mathrm{dM} 6.0$ & $27.1126 \pm 0.1581$ & $16.296 \pm 0.033$ & $0.096 \pm 0.005$ & & - & Subdwarf? \\
\hline PM J00115+5908 & 15.611 & 2.121 & 2887 & 2906 & $2896 \pm 10$ & $\mathrm{dM} 6.0$ & $107.4214 \pm 0.0651$ & $15.766 \pm 0.021$ & $0.124 \pm 0.008$ & & - & \\
\hline PM J10430-0912 & 15.071 & 1.963 & 2985 & 2951 & $2968 \pm 17$ & dM5.5 & $81.3994 \pm 0.2614$ & $14.624 \pm 0.027$ & $0.177 \pm 0.015$ & & - & Binary? \\
\hline & & & & & dM7 sample & & & & & & & \\
\hline GJ 1111 & 14.855 & 2.339 & 2800 & 2786 & $2793 \pm 7$ & $\mathrm{dM} 7.2$ & $279.2901 \pm 0.1345$ & $17.085 \pm 0.021$ & $0.086 \pm 0.006$ & -0.13 & 3 & \\
\hline GJ 3517 & 18.959 & 2.252 & 2832 & 2582 & $2707 \pm 125$ & $\mathrm{dM} 8.3$ & $115.3036 \pm 0.1132$ & $19.268 \pm 0.022$ & $0.040 \pm 0.019$ & +0.21 & 3 & \\
\hline GJ 3622 & 15.60 & 2.34 & 2800 & 2717 & $2758 \pm 41$ & $\mathrm{dM} 7.7$ & $219.1159 \pm 0.1567$ & $17.303 \pm 0.022$ & $0.085 \pm 0.014$ & -0.26 & 5 & \\
\hline GJ 3877 & 17.141 & 2.39 & 2781 & 2700 & $2740 \pm 40$ & $\mathrm{dM} 7.9$ & $141.6865 \pm 0.1063$ & $17.898 \pm 0.022$ & $0.068 \pm 0.011$ & & 8 & \\
\hline GJ 4281 & 17.08 & 2.34 & 2800 & - & $2800 \pm 54$ & $\mathrm{dM} 7.2$ & $91.8949 \pm 0.0948$ & $16.896 \pm 0.022$ & $0.092 \pm 0.019$ & & 4 & \\
\hline Gl $65 \mathrm{~B}$ & 12.81 & 2.22 & 2845 & - & $2845 \pm 54$ & $\mathrm{dM} 6.6$ & $372.1631 \pm 0.2803$ & $15.664 \pm 0.022$ & $0.146 \pm 0.023$ & -0.21 & 7 & PMS? \\
\hline Gl 283B & 16.696 & 2.25 & 2833 & 2530 & $2681 \pm 151$ & $\mathrm{dM} 8.6$ & $109.0542 \pm 0.0827$ & $16.884 \pm 0.022$ & $0.127 \pm 0.074$ & & . & \\
\hline Gl 406 & 13.507 & 2.213 & 2848 & 2812 & $2830 \pm 18$ & $\mathrm{dM} 6.8$ & $418.3 \pm 2.5$ & $16.614 \pm 0.033$ & $0.098 \pm 0.010$ & & 52 & \\
\hline Gl $644 \mathrm{C}$ & 16.916 & 2.428 & 2767 & 2745 & $2756 \pm 11$ & $\mathrm{dM} 7.7$ & $153.8139 \pm 0.1148$ & $17.851 \pm 0.022$ & $0.067 \pm 0.005$ & -0.14 & 5 & \\
\hline Gl 752B & 17.370 & 2.283 & 2821 & 2670 & $2745 \pm 75$ & $\mathrm{dM} 7.8$ & $168.9620 \pm 0.1299$ & $18.509 \pm 0.022$ & $0.051 \pm 0.014$ & +0.05 & 8 & \\
\hline LHS $325 \mathrm{~A}$ & 18.67 & 2.24 & 2836 & - & $2836 \pm 54$ & $\mathrm{dM} 6.7$ & $47.5722 \pm 0.1323$ & $17.057 \pm 0.026$ & $0.079 \pm 0.015$ & & - & \\
\hline LHS 2351 & 19.560 & 2.34 & 2800 & 2710 & $2755 \pm 45$ & $\mathrm{dM} 7.7$ & $46.8910 \pm 0.1620$ & $17.915 \pm 0.027$ & $0.065 \pm 0.012$ & -0.44 & - & \\
\hline LHS 2397 & 19.24 & 2.300 & 2814 & 2700 & $2757 \pm 57$ & $\mathrm{dM} 7.7$ & $18.9008 \pm 0.1590$ & $15.622 \pm 0.038$ & $0.186 \pm 0.043$ & & - & Binary? \\
\hline LSPM J0253+1652 & 15.133 & 2.404 & 2776 & 2719 & $2747 \pm 28$ & $\mathrm{dM} 7.8$ & $261.0147 \pm 0.2690$ & $17.216 \pm 0.022$ & $0.092 \pm 0.012$ & & - & \\
\hline
\end{tabular}


${ }^{1}$ From the $(\mathrm{R}-\mathrm{I})_{C}$ color.

${ }^{2}$ Compiled from the literature.

${ }^{3}$ Mean effective temperature. 
Table 3:: The list of effective temperatures from some authors (Morales et al. 2008,

Jenkins et al. 2009, Wright et al. 2011, Stelzer et al. 2013, Lepine et al. 2013 ,

Gaidos et al. 2014) for our sample of dM4 stars.

\begin{tabular}{|c|c|c|c|c|c|c|c|}
\hline Star & $\begin{array}{c}T_{\text {eff }}{ }^{1} \\
\text { (K) } \\
\text { This Work }\end{array}$ & $\begin{array}{c}T_{\text {eff }} \\
(\mathrm{K}) \\
\text { Morales } 2008\end{array}$ & $\begin{array}{c}T_{\text {eff }} \\
(\mathrm{K}) \\
\text { Jenkins } 2009\end{array}$ & $\begin{array}{c}T_{\text {eff }} \\
(K) \\
\text { Wright } 2011\end{array}$ & $\begin{array}{c}T_{\text {eff }} \\
(\mathrm{K}) \\
\text { Stelzer } 2013\end{array}$ & $\begin{array}{c}T_{\text {eff }} \\
(\mathrm{K}) \\
\text { Lepine } 2013\end{array}$ & $\begin{array}{c}T_{\text {eff }} \\
(\mathrm{K}) \\
\text { Gaidos } 2014\end{array}$ \\
\hline GJ & 3297 & - & - & - & - & - & - \\
\hline GJ 1001A & 3297 & - & - & - & - & - & 3351 \\
\hline GJ $1005 \mathrm{AB}$ & 3315 & - & - & - & - & - & 3227 \\
\hline GJ $1006 \mathrm{~A}$ & 3273 & - & - & - & - & 3250 & 3298 \\
\hline GJ 1006B & 3263 & - & - & - & - & 3100 & 3211 \\
\hline GJ 1013 & 3304 & - & - & - & - & - & 3243 \\
\hline GJ 1034 & 3250 & - & 3035 & - & - & - & - \\
\hline GJ 1092 & 3251 & 3093 & - & - & - & - & - \\
\hline GJ 1105 & 3315 & - & - & - & 3241 & 3280 & 3197 \\
\hline GJ 1129 & 3246 & - & - & - & - & - & 3209 \\
\hline GJ 1134 & 3223 & - & 2996 & - & - & 3100 & 3158 \\
\hline GJ 1138 & 3233 & - & - & - & 3089 & 3140 & 3161 \\
\hline GJ 1174 & 3317 & - & - & - & - & 3230 & 3201 \\
\hline GJ 1207 & 3270 & - & - & - & 3241 & - & 3206 \\
\hline GJ $1218 \mathrm{AB}$ & 3304 & - & - & - & - & - & 3281 \\
\hline GJ 1222 & 3291 & - & - & - & - & 3100 & 3162 \\
\hline GJ 1235 & 3231 & - & - & - & 3089 & 3060 & 3099 \\
\hline GJ 1254 & 3287 & - & - & - & - & 3280 & 3226 \\
\hline GJ 1263 & 3336 & - & - & - & - & - & 3252 \\
\hline GJ 1265 & 3231 & - & - & - & - & - & 3023 \\
\hline GJ 1270 & 3254 & - & - & - & - & 3270 & 3133 \\
\hline GJ 1289 & 3223 & - & 2969 & - & 3165 & 3100 & 3155 \\
\hline GJ 2043B & 3246 & 3130 & - & - & - & - & - \\
\hline GJ 2069A & 3263 & - & - & - & - & 3100 & 3374 \\
\hline GJ 3149B & 3263 & 3190 & - & - & - & - & 3209 \\
\hline GJ 3198 & 3338 & - & - & - & - & - & 3447 \\
\hline GJ 3235 & 3302 & - & - & - & - & - & 3256 \\
\hline GJ $3263 \mathrm{AB}$ & 3340 & - & - & - & - & - & 3290 \\
\hline GJ $3322 \mathrm{~A}$ & 3283 & - & - & - & - & - & 3348 \\
\hline GJ 3374B & 3330 & - & - & - & - & - & 3240 \\
\hline GJ 3466 & 3208 & - & - & - & - & - & 3100 \\
\hline GJ $3522 \mathrm{AB}$ & 3254 & - & - & - & 3241 & 3140 & 3170 \\
\hline GJ $3577 \mathrm{~A}$ & 3304 & - & - & 3500 & - & - & - \\
\hline GJ 3612 & 3343 & - & - & - & - & 3290 & 3440 \\
\hline GJ $3631 \mathrm{AB}$ & 3246 & - & 2966 & - & - & - & - \\
\hline GJ 3666 & 3325 & - & - & - & - & 3250 & 3239 \\
\hline GJ 3707 & 3273 & - & 3033 & - & - & - & 3270 \\
\hline GJ 3764 & 3351 & - & - & - & - & - & 3473 \\
\hline GJ 3779 & 3278 & - & - & - & - & 3130 & 3259 \\
\hline GJ 3780 & 3288 & - & - & - & - & - & 3320 \\
\hline GJ 3789 & 3263 & - & 2951 & 3407 & - & 3150 & 3389 \\
\hline GJ 3800 & 3216 & - & - & - & - & - & 3265 \\
\hline GJ 3801 & 3336 & 3240 & - & - & 3241 & 3280 & 3157 \\
\hline GJ 3804 & 3348 & 3300 & - & - & - & - & 3274 \\
\hline GJ 3839 & 3224 & - & - & - & - & 3130 & 3242 \\
\hline GJ 3843 & 3353 & - & - & - & - & - & 3283 \\
\hline GJ 3873 & 3272 & - & - & - & - & 3240 & 3403 \\
\hline GJ 3900 & 3283 & - & - & - & - & - & 3300 \\
\hline GJ 3919 & 3269 & - & - & - & - & 3100 & 3238 \\
\hline
\end{tabular}


Table 3:: continued.

\begin{tabular}{|c|c|c|c|c|c|c|c|}
\hline Star & $\begin{array}{c}T_{\text {eff }}{ }^{1} \\
\text { (K) } \\
\text { This Work }\end{array}$ & $\begin{array}{c}T_{\text {eff }} \\
(\mathrm{K}) \\
\text { Morales } 2008\end{array}$ & $\begin{array}{c}T_{\text {eff }} \\
(\mathrm{K}) \\
\text { Jenkins } 2009\end{array}$ & $\begin{array}{c}T_{\text {eff }} \\
(\mathrm{K}) \\
\text { Wright } 2011\end{array}$ & $\begin{array}{c}T_{\text {eff }} \\
(K) \\
\text { Stelzer } 2013\end{array}$ & $\begin{array}{c}T_{\text {eff }} \\
(\mathrm{K}) \\
\text { Lepine } 2013\end{array}$ & $\begin{array}{c}T_{\text {eff }} \\
(\mathrm{K}) \\
\text { Gaidos } 2014\end{array}$ \\
\hline GJ $4063 \mathrm{AB}$ & 3315 & - & 3265 & - & - & - & 3342 \\
\hline GJ 4185B & 3304 & - & - & - & - & 3260 & 3302 \\
\hline GJ 4186B & 3293 & - & - & - & - & - & 3302 \\
\hline GJ 4207 & 3341 & - & - & - & - & - & 3340 \\
\hline GJ 4333 & 3258 & 3150 & - & - & - & 3280 & 3241 \\
\hline GJ 4338B & 3246 & - & - & - & - & 3150 & 3311 \\
\hline GJ 4367 & 3248 & - & - & - & - & - & 3221 \\
\hline GJ 4378A & 3293 & - & - & - & - & - & 3313 \\
\hline GJ $4387 \mathrm{AB}$ & 3309 & - & - & - & - & - & 3255 \\
\hline GJ 9652B & 3309 & 3260 & - & - & - & - & 3452 \\
\hline Gl 15B & 3270 & - & - & - & 3241 & 3260 & 3282 \\
\hline Gl 46 & 3338 & 3260 & - & - & - & - & 3472 \\
\hline Gl 54.1 & 3188 & - & - & - & 3089 & - & 3062 \\
\hline Gl $84.1 \mathrm{~B}$ & 3348 & 3190 & - & - & - & - & 3400 \\
\hline Gl 102 & 3194 & - & - & - & 3165 & 3130 & 3190 \\
\hline Gl 105B & 3254 & 3130 & 3042 & - & 3165 & 3130 & 3198 \\
\hline Gl 166C & 3239 & 3070 & - & 3301 & - & - & - \\
\hline GL $169.1 \mathrm{~A}$ & 3259 & 3272 & 3000 & - & 3165 & 3100 & 3277 \\
\hline GL 179 & 3352 & - & - & - & - & 3260 & 3457 \\
\hline Gl $206 \mathrm{AB}$ & 3336 & - & 3137 & - & - & 3200 & 3292 \\
\hline GL 213 & 3242 & - & - & - & 3165 & 3100 & 3122 \\
\hline GL 232 & 3207 & - & 3019 & - & 3089 & 3060 & 3099 \\
\hline GL $268 \mathrm{AB}$ & 3192 & 3184 & - & - & 3089 & 3130 & 3069 \\
\hline GL 273 & 3294 & - & 3093 & - & 3089 & 3130 & 3317 \\
\hline Gl 277B & 3302 & - & 3079 & - & - & 3400 & 3604 \\
\hline GL 285 & 3196 & - & 2975 & 3482 & 3089 & 3130 & 3242 \\
\hline GL 299 & 3187 & - & 3036 & - & 3089 & 3050 & 3152 \\
\hline GL 300 & 3206 & - & - & - & 3165 & - & 3212 \\
\hline GL 317 & 3348 & - & - & - & - & - & 3400 \\
\hline GL 319B & 3325 & - & - & - & - & - & 3555 \\
\hline GL $324 \mathrm{~B}$ & 3226 & 3130 & - & - & - & 3100 & 3166 \\
\hline GL 347B & 3246 & 3080 & - & - & - & - & 3498 \\
\hline GL 375A & 3344 & 3260 & - & 3834 & - & - & 3354 \\
\hline GL 398 & 3328 & - & - & - & - & 3140 & 3367 \\
\hline Gl 402 & 3099 & - & 3038 & - & 3165 & 3100 & 3238 \\
\hline Gl 431 & 3354 & 3300 & - & 3580 & - & - & 3399 \\
\hline Gl 445 & 3328 & 3240 & 3137 & - & 3241 & 3300 & 3186 \\
\hline Gl 447 & 3198 & - & 2966 & - & 3165 & 3130 & 3145 \\
\hline Gl 452.1 & 3354 & - & - & - & - & 3270 & 3298 \\
\hline GL 458B & 3325 & 3320 & - & - & - & - & - \\
\hline Gl 469 & 3329 & 3260 & - & - & - & 3180 & 3239 \\
\hline GL 486 & 3313 & 3240 & 3086 & - & 3241 & 3290 & 3270 \\
\hline GL 490B & 3263 & 3130 & 3055 & - & - & 3150 & 3266 \\
\hline GL 512B & 3225 & 3130 & - & - & - & - & 3577 \\
\hline GL $520 \mathrm{C}$ & 3273 & 3150 & - & - & - & - & - \\
\hline GL 545 & 3321 & - & - & - & - & - & 3253 \\
\hline Gl 553.1 & 3355 & 3260 & - & - & - & - & 3231 \\
\hline GL 555 & 3240 & 3150 & 2984 & - & 3165 & - & 3211 \\
\hline GL 568AB & 3312 & 3240 & - & - & - & 3280 & 3185 \\
\hline Gl 590 & 3252 & - & - & - & - & - & 3103 \\
\hline GL 592 & 3293 & - & - & - & - & - & 3263 \\
\hline GL 609 & 3263 & - & - & - & 3165 & 3100 & 3105 \\
\hline
\end{tabular}


Table 3:: continued.

\begin{tabular}{|c|c|c|c|c|c|c|c|}
\hline Star & $\begin{array}{c}T_{\text {eff }}{ }^{1} \\
\text { (K) } \\
\text { This Work }\end{array}$ & $\begin{array}{c}T_{\text {eff }} \\
(\mathrm{K}) \\
\text { Morales } 2008\end{array}$ & $\begin{array}{c}T_{\text {eff }} \\
(\mathrm{K}) \\
\text { Jenkins } 2009\end{array}$ & $\begin{array}{c}T_{\text {eff }} \\
(\mathrm{K}) \\
\text { Wright } 2011\end{array}$ & $\begin{array}{c}T_{\text {eff }} \\
(K) \\
\text { Stelzer } 2013\end{array}$ & $\begin{array}{c}T_{\text {eff }} \\
(\mathrm{K}) \\
\text { Lepine } 2013\end{array}$ & $\begin{array}{c}T_{\text {eff }} \\
(\mathrm{K}) \\
\text { Gaidos } 2014\end{array}$ \\
\hline GL $630.1 \mathrm{~A}$ & 3204 & - & 2887 & - & - & 3100 & 3073 \\
\hline GL 643 & 3305 & 3210 & 3104 & - & - & 3241 & 3438 \\
\hline Gl $669 \mathrm{~A}$ & 3319 & - & 3104 & 3660 & - & 3260 & 3426 \\
\hline GL 682 & 3268 & 3190 & - & - & - & 3241 & 3190 \\
\hline GL 695B & 3345 & 3300 & - & - & - & 3241 & - \\
\hline Gl 699 & 3266 & - & - & - & 3165 & 3100 & 3237 \\
\hline GL $720 \mathrm{~B}$ & 3304 & 3210 & - & - & - & 3200 & 3239 \\
\hline GL 725B & 3336 & - & 3172 & - & 3241 & 3290 & 3334 \\
\hline GL 729 & 3276 & 3240 & - & 3191 & 3241 & - & 3213 \\
\hline GL $732 \mathrm{~A}$ & 3293 & - & - & - & - & - & 3207 \\
\hline GL 766AB & 3223 & - & - & - & - & 3100 & 3278 \\
\hline GL 781.1B & 3274 & 3190 & - & - & - & - & 3412 \\
\hline GL 783.2B & 3247 & 3150 & - & - & - & - & - \\
\hline GL 791.2A & 3212 & - & 2896 & 3264 & 3089 & 2980 & 3128 \\
\hline GL $810 \mathrm{~A}$ & 3249 & - & - & - & - & - & 3253 \\
\hline GL $812 \mathrm{~A}$ & 3319 & - & 3141 & - & - & - & 3399 \\
\hline Gl 865A & 3337 & 3280 & - & - & - & - & 3396 \\
\hline Gl 865B & 3337 & 3280 & - & - & - & - & 3326 \\
\hline GL 873A & 3325 & 3260 & 3168 & 3521 & 3241 & 3270 & 3262 \\
\hline GL $876 \mathrm{~A}$ & 3304 & - & 3172 & - & 3165 & - & 3321 \\
\hline Gl 896A & 3358 & 3280 & 3090 & 3328 & 3089 & 3290 & 3375 \\
\hline Sum & & 121949 & 91596 & 38068 & 104369 & 190843 & 363121 \\
\hline No meas & & 38 & 30 & 11 & 32 & 60 & 111 \\
\hline Mean & & 3209.18 & 3053.2 & 3460.73 & 3261.53 & 3180.72 & 3271.36 \\
\hline Sum (R-I)c & & 125150 & 97951 & 36190 & 104069 & 196308 & 364322 \\
\hline Mean & & 3293.42 & 3265.03 & 3290.0 & 3252.16 & 3271.8 & 3282.18 \\
\hline Diff. & & -84.24 & -211.83 & +170.73 & $\begin{array}{l}+9.37 \\
\end{array}$ & -91.08 & -10.82 \\
\hline Diff. \% & & $-2.56 \%$ & $-6.49 \%$ & $+5.19 \%$ & $+0.29 \%$ & $-2.78 \%$ & $-0.33 \%$ \\
\hline
\end{tabular}


${ }^{1}$ From the $(\mathrm{R}-\mathrm{I})_{C}$ color. 